\title{
SMAD TRANSCRIPTION FACTOR EXPRESSION IN ANOXIA-TOLERANT TRACHEMYS SCRIPTA ELEGANS AND DEHYDRATION-TOLERANT XENOPUS LAEVIS: A COMPARATIVE STUDY
}

\author{
BY \\ Ryan Abdul-Rahman Girgrah \\ B.Sc. Hons. (Chemistry) \\ Carleton University, 2013
}

\begin{abstract}
A THESIS SUBMITTED TO THE FACULTY OF GRADUATE AND POSTDOCTORAL AFFAIRS IN PARTIAL FULFILLMENT OF THE REQUIREMENTS FOR THE DEGREE OF
\end{abstract}

MASTER OF SCIENCE

CHEMISTRY

CARLETON UNIVERSITY

OTTAWA, ONTARIO, CANADA

(C) COPYRIGHT 2015

RYAN A.-R. GIRGRAH 
We, the undersigned, hereby recommend to the Faculty of Graduate Studies and Postdoctoral Affairs acceptance of this thesis:

\title{
SMAD TRANSCRIPTION FACTOR EXPRESSION IN ANOXIA-TOLERANT TRACHEMYS SCRIPTA ELEGANS AND DEHYDRATION-TOLERANT XENOPUS LAEVIS: A COMPARATIVE STUDY
}

\author{
submitted by \\ Ryan A.-R. Girgrah, B.Sc. Hons.
}

in partial fulfillment of the requirements for the degree of Master of Science, Chemistry.

Chair, Department of Chemistry

Thesis Supervisor

Carleton University 


\begin{abstract}
The TGF- $\beta$ signaling pathway with its associated transcription factors (SMADs) was studied in the red-eared slider (Trachemys scripta elegans; liver, heart, white skeletal muscle, red skeletal muscle, and kidney) as a model for anoxia tolerance and the African clawed frog (Xenopus laevis; liver and muscle) as a model for dehydration resistance. In addition, protein expression levels of precursor cytokines were measured, as well as known genes and proteins downstream of the SMAD signaling pathway, such as c-Myc, and AP1 proteins. The data showed that selective control of different SMAD isoforms occurred in response to environmental stress, the mechanism being reversible protein phosphorylation. Cellular responses and signaling profiles were observed to be highly tissue-specific and stress-specific. SMADs were shown to play a role in the hypometabolic response of both animal models through the regulation of cell cycle arrest, angiogenic processes, and oxidative injury management strategies.
\end{abstract}




\section{ACKNOWLEDGEMENTS}

Animal blood, hockey sweat, and undergraduate tears.

Before I begin to thank all the Storey lab members I must first thank Dr. Storey as he introduced me to the potential for graduate school and the value of molecular biology to my intended career plans. I highly doubt I would be where I am today if not for his acceptance to his well-oiled machine of a lab, nor the countless snippets of advice from simply occupying a space in the large office while he lectured and divulged the details of his life stories to all those graduate students present. These proved to shift my way of thinking and improve my critical thinking skills. If the lab is a machine, then Janet Storey is the lead engineer that designed it that way and maintains its function. Without her discussions to pave the way, I would still be a lost chemist in a seemingly vast and unnavigable ocean of biology. She has provided countless, invaluable edits and suggestions to all my writing specimens. Her evolutionary patience was certainly exercised undoubtedly during my two year tenure while completing this work.

I want to acknowledge the entire Storey Lab as whole, even if not mentioned by name, for accepting me into their family and making me a part of their traditions. Of the students and colleagues comprising "The Family", I would like to start by thanking my first mentor in the Storey Lab, Kama Szereszewski. She showed me the art of western blotting and prodded me forward into the unknown. Next is Sanoji Wijenayake whom showed me how to operate the (very expensive) Luminex multiplex instrument (known by the Lab as the immunoblotter on steroids). Beyond that she gave feedback on blots that allowed the untrained eye to begin to diagnose and troubleshoot problematic immunoblotting conditions and antibodies. Bryan "BLuu" Luu offered his blessings to this new graduate student and steered him clear of sure danger and gave me his insights into a broad range of topics from food to politics, as well the frequent excursions to the heart of Canada's capital and its eateries - these typically featured an appearance by Tony "Tidus" "Tobicus" "Toby” Zhang. Hanane "Hananigans" Hadj-Moussa delivered a source of entertainment, distraction from daily frustrations via regular coffee breaks, and partner in comedic crime. Mike Smolinski and Anthony Ruberto introduced me to 
possibly best worst films 'The Room' and 'MacGruber', and formulated the ever required strain-reliever JoneZenol ${ }^{\mathrm{TM}}$. Rasha Al-Attar provided constant nutrition and new ideas for projects and continues to be a role model for what a graduate student should be. She taught me that perseverance (and a bit of stubbornness) is ultimately rewarded - enjoy those mice.

There remains a very special individual that truly inspired me every day to be successful and provided unlimited encouragement and advice. For that I am indebted to this person for without them I would not have realized my potential and to pursue further work in academia that ultimately gave me a pathway to follow and countless possibilities.

I would also like to extend my gratitude to the former (read: epic) Storey Lab members Dr. Neal Dawson, Dr. Barbara Katzenback, Dr. Shannon Tessier and Dr. Jing Zhang for their early and often support and helping me get back on my feet whenever I lost my footing.

Finally, I want to thank my family and friends to whom this thesis is dedicated. Despite their complete lack of understanding what it is that I have dedicated incalculable hours of time on, Maher and Wafa Girgrah have encouraged me and provided me the foundation to stand upon. From childhood until present-day, I have had one of the greatest friends that any person could ask for in Yasser "P." Ghazi who has had an immense influence in shaping me (for the past 16 years) into the person I am today.

It would seem to be an injustice to not mention the educators that fostered my initial interest in the field of chemistry and guided my education into an appreciable knowledge base that I still use in my academic career. I would like to thank Mrs. Margaret Granville whose quirky teachings ignited my initial interest in chemistry in Grade 11. Mr. Lawrence Burns continued to further my knowledge in the field and provided many hands-on experiences that really lead to an honest admiration for this craft. His excellent tutelage prepared me for post-secondary studies in a way that was by my fellow first-year cohort's account rare.

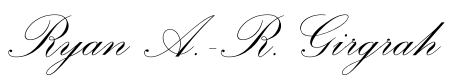




\section{TABLE OF CONTENTS}

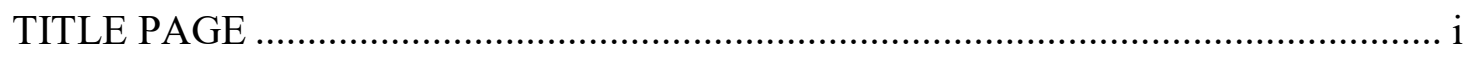

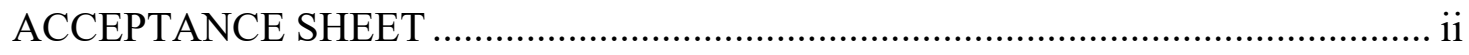

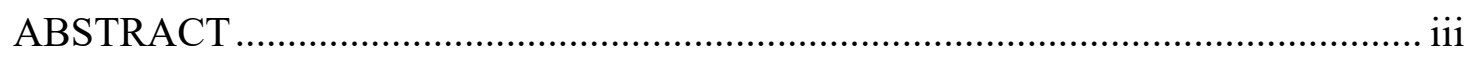

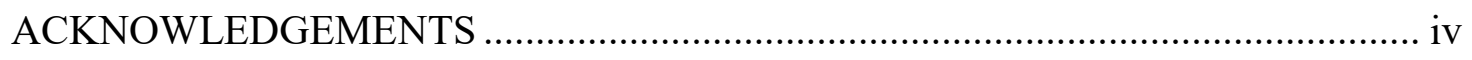

TABLE OF CONTENTS …………………................................................ vi

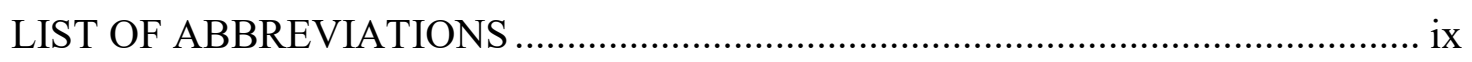

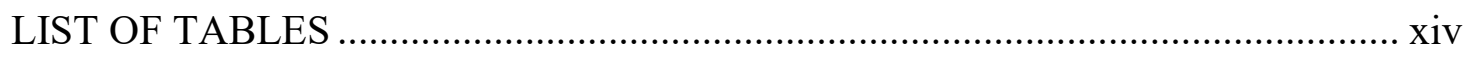

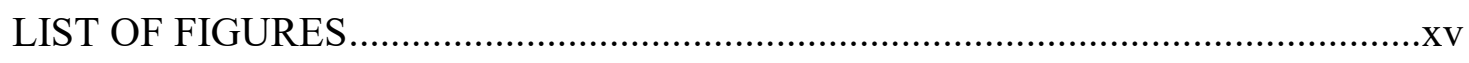

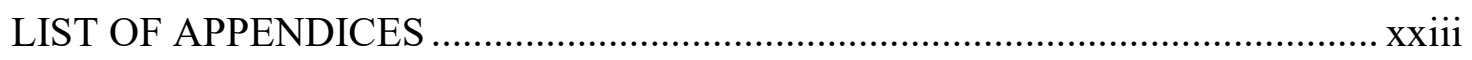

Chapter 1: General Introduction ...........................................................................1

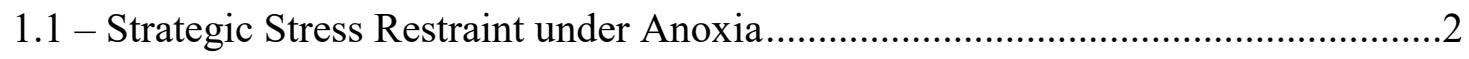

1.2 - Desert Dehydration Determinations............................................................

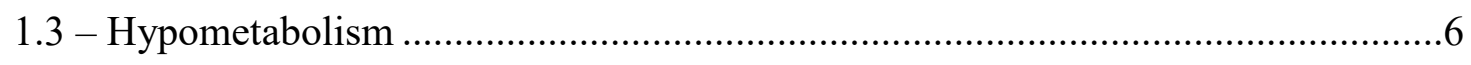

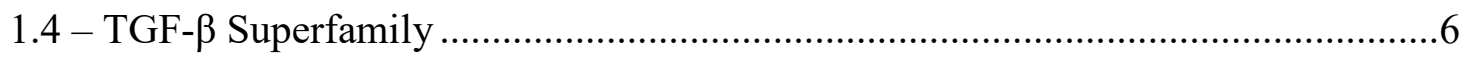

1.5 - Objectives and Hypotheses .........................................................................

1.5.1 - Objective 1: TGF- $\beta$ pathway, the Mediator for Cellular Metabolic Rate Depression ………………................................................................11

1.5.2 - Objective 2: Maintaining Homeostasis in Tissues Through the TGF- $\beta$

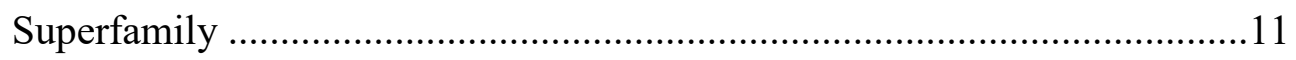

1.5.3 - Objective 3: TGF- $\beta$ as an Important Liaison in Dehydration Survival .........12

1.6 - General Introduction Figures …………………...............................................14

\section{Chapter 2: Effects of Anoxic Exposure on SMAD and its Downstream} Protein Expression in Trachemys scripta elegans ......................................19

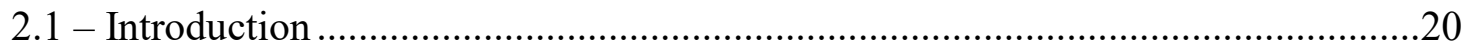

2.1.1 - Annual Anoxia Anomaly .......................................................................20

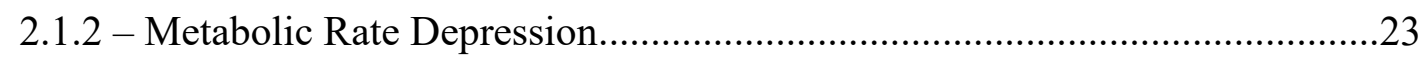

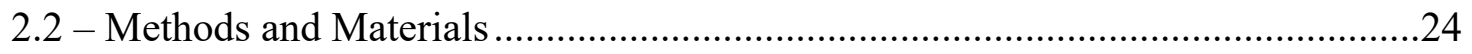

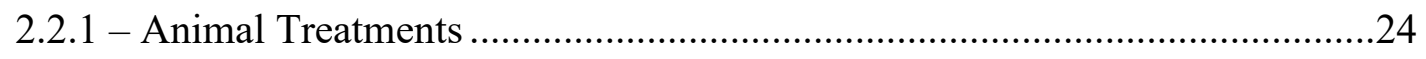

2.2.2 - Homogenization of Animal Tissues and Total Protein Isolation .................25 
2.2.3 - SDS Polyacrylamide Gel Electrophoresis and Immunoblotting …..............26

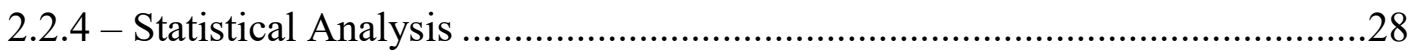

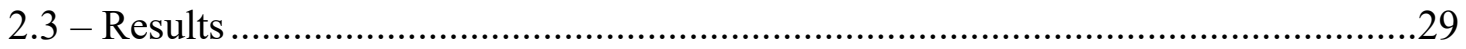

2.3.1 - SMAD Liver Expression in Anoxic Conditions.........................................29

2.3.2 - SMAD White Muscle Expression in Anoxic Conditions................................36

2.3.3 - SMAD Red Muscle Expression in Anoxic Conditions ..................................43

2.3.4 - SMAD Kidney Expression in Anoxic Conditions........................................50

2.3.5 - SMAD Heart Expression in Anoxic Conditions …………………..............56

2.3.6 - TGF- $\beta$ Superfamily Up- and Downstream Protein Expression.....................63

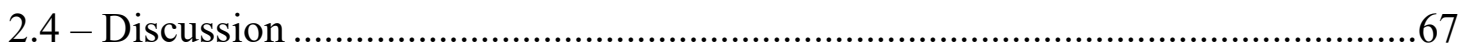

2.4.1 - TGF- $\beta$ Superfamily Mediated Effects in TSE Liver ....................................68

2.4.2 - TGF- $\beta$ Superfamily Mediated Effects in TSE White Muscle ........................72

2.4.3 - TGF- $\beta$ Superfamily Mediated Effects in TSE Red Muscle ..........................75

2.4.4 - TGF- $\beta$ Superfamily Mediated Effects in TSE Kidney ..................................77

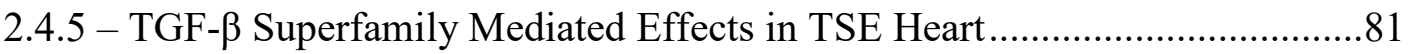

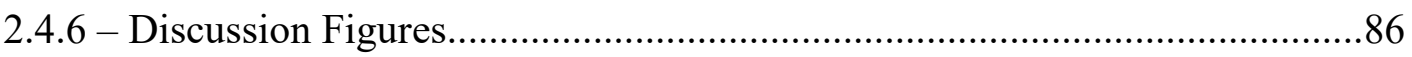

\section{Chapter 3: Effects of Dehydration Stress on SMAD and its Downstream Protein Expression in Xenopus laevis. ..................................................90}

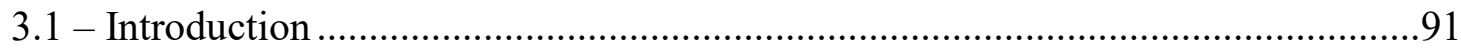

3.1.1 - Dehydration Stress and Aestivation ..........................................................91

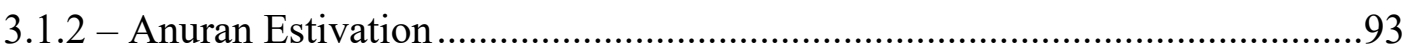

3.1.3 - Metabolic Rate Depression in Aestivation ..................................................94

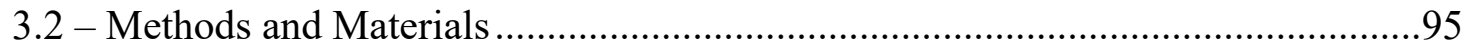

3.2.1 - Animal Treatments ..................................................................................95

3.2.2 - Homogenization of Animal Tissues and Total Protein Isolation ..................96

3.2.3 - SDS Polyacrylamide Gel Electrophoresis and Immunoblotting ..................97

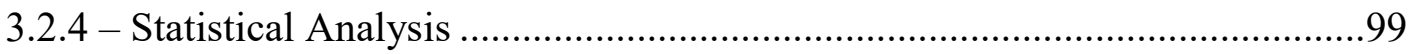

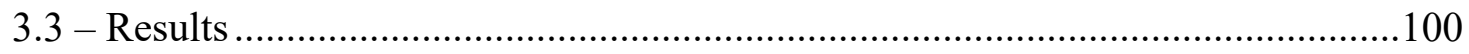

3.3.1 - Effects of Dehydration on SMAD Expression in Liver ...............................100

3.3.2 - Effects of Dehydration on SMAD Expression in Muscle ...........................107

3.3.3 - Downstream Targets of the TGF- $\beta$ Pathway in Liver .................................114

3.3.4 - Downstream Targets of the TGF- $\beta$ Pathway in Muscle .............................118

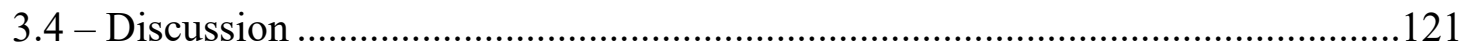

3.4.1 - X. laevis Tissues Cease Cell Cycle during Dehydration ............................121 
3.4.2 - X. laevis Tissues Experience Oxidative Stress under Dehydration

3.4.3 - AP-1 Proteins and Their Role in X. laevis Tissues.

3.4.4 - Discussion Figures.

Chapter 4: General Discussion ..............................................................130

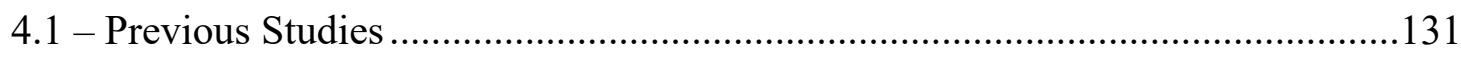

4.2 - Anoxic Effects on SMAD Protein Expression and Cellular Processes..............133

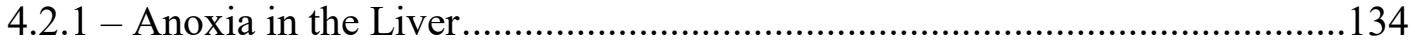

4.2.2 - Anoxia in White Skeletal Muscle...........................................................134

4.2.3 - Anoxia in Red Skeletal Muscle ...............................................................135

4.2.4 - Anoxia in Kidney ............................................................................... 136

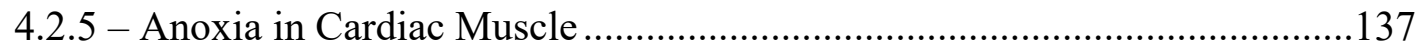

4.3 - Dehydration Effects on SMAD Protein Expression and Cellular Processes .....138

4.4 - Physiological Differences in TGF- $\beta$ Signaling .......................................... 140

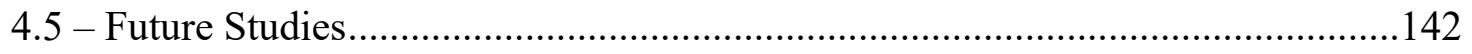

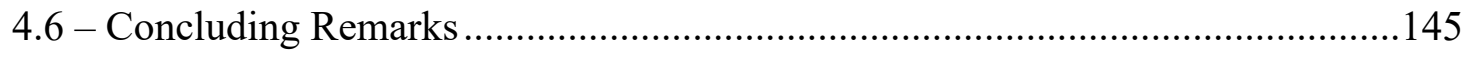

4.7 - General Discussion Figures............................................................. 146

References.............................................................................................148

APPENDIX A: Animal Specific Gene and Protein Sequences ...............168

APPENDIX B: Immunoblotting Conditions............................................174

APPENDIX C: List of Antibodies.........................................................182

APPENDIX D: List of Epitopes of Specific Antibodies ............................184

APPENDIX E: Ensuring Antibody Specificity and Band Selectivity for

Immunoblotting ....................................................................................................186

APPENDIX F: Phylogenetic Trees of SMADs and Related TGF- $\beta$

Superfamily Proteins .........................................................................188

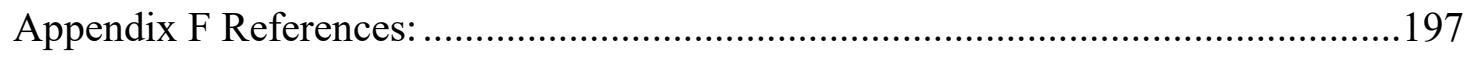

APPENDIX G: SMAD Isoforms: Roles and Controls ..............................198 


\section{LIST OF ABBREVIATIONS}

20hA. 20 hour anoxia $5 \mathrm{hA}$ 5 hour anoxia

$\gamma$-GCS.

$\mu \mathrm{g}$

$\mu \mathrm{L}$ 4-(2-aminoethyl)-benzenesulfonylfluoride hydrochloride Akt protein kinase $\mathrm{B}$ ALK-1 activin receptor-like kinase-1 AP-1 adaptor protein 1 APS ammonium persulphate

ARE antioxidant response element ATF .activating/cAMP-dependent transcription factor ATP .adenosine triphosphate BMP bone morphogenic protein BMPRI (I) BMP receptor I (I) BSA bovine serum albumen bZIP basic region-leucine zipper protein c-Fos proto-oncogene, FBJ murine osteosarcoma viral oncogene c-Jun. proto-oncogene protein, member of the Jun family, coded by the JUN gene c-Myc .proto-oncogene protein, coded by the MYC gene cAMP cyclical 3'-adenosine monophosphate CAT catalase CBP CREB-binding protein $\mathrm{CDC}$ cell division/dependent cycle protein

CDK cyclin-dependent kinase

$\mathrm{CH}_{3} \mathrm{COOH}$ acetic acid $\mathrm{cm}$ centimetre $\left(10^{-2}\right.$ metres $)$

CREB cAMP response element-binding protein 
CTSE

CXL

$\mathrm{ddH}_{2} \mathrm{O}$

DNA

EC

ECL

EDTA

ELISA

EndMT .

ERK

$\mathrm{FADH}_{2}$

FGF

FoxO

$\mathrm{G}_{1}$

$\mathrm{G}_{2}$

GDF

GSH

GSSG

GST

h.

$\mathrm{H}_{2} \mathrm{O}_{2}$

HD

HEPES

HIF-1

HLH

HMW-PVA

HO-1

HRP

Id1

IgG control Trachemys scripta elegans control Xenopus laevis distilled deionized water deoxyribonucleic acid epithelial cells enhanced chemiluminescence .ethylenediamine tetraacetate enzyme-linked immunosorbent assay endothelial-to-mesenchymal transition extracellular signal-regulated kinase flavin adenine dinucleotide .. fibroblast growth factor forkhead box, class O protein gap phase 1 gap phase 2 growth differentiation factor glutathione, reduced form glutathione (disulphide), oxidized form glutathione S-transferase hour(s) hydrogen peroxide high dehydration/desiccation 4-(2-hydroxyethyl)-1-piperazineethanesulfonicacid ..hypoxia-inducible factor 1 helix-loop-helix high molecular weight PVA heme oxygenase 1 ..horseradish peroxidase inhibitor of differentiation/DNA binding 1 .immunoglobulin $\mathrm{G}$ 
$\mathrm{kDa}$ kilodalton ( $10^{3}$ daltons)

LMW-PVA low molecular weight PVA $\mathrm{mA}$ milliamperes $\left(10^{-3}\right.$ coulomb per second)

$\mathrm{M}$ mitosis

MAD MAPK

MD MEGA $\mathrm{MeOH}$ $\mathrm{MeSH}$ $\mathrm{MH} 1$ $\mathrm{MH} 2$ $\min$. miRNA $\mathrm{mL}$ $\mathrm{mM}$ MRD mRNA mTOR MW $\mathrm{NADH}$ nicotinamide adenine dinucleotide, reduced form NCBI National Center for Biotechnology Information Nkx2-5 NK2 homeobox 5 $\mathrm{nm}$ $\mathrm{Nrf} 2$ nanometre $\left(10^{-9}\right.$ metres $)$ $\mathrm{O} / \mathrm{N}$ p1 $15^{\operatorname{Ink} 4 \mathrm{~B}}$ $\mathrm{p} 16^{\operatorname{Ink} 4 \mathrm{~A}}$ cyclin-dependent kinase inhibitor 2A (CDKN2A) p2 $1^{\text {Cip } 1 / \text { Waf } 1}$ cyclin-dependent kinase inhibitor 1 (CDK-interacting protein) p2 $7^{\text {Kip1 }}$ cyclin-dependent kinase inhibitor 1B 
PAGE

PAI-1

PI3K

PMSF

PVA.

PVDF

RBC

Ras.

RNA

ROS

$\mathrm{s}$.

$\mathrm{S}$

SARA

SBE

SDS

S.E.M.

SERPINE1

SMA

SMAD

SMC.

SOD

T $\beta R I(I)$

TBST

TEMED

Tf..

TGF- $\beta$.

TPA

Tris

..12-O-tetradecanoylphorbol-13-acetate, or tetradecanoylphorbol acetate

TSC22D 3

TSE

polyacrylamide gel electrophoresis .plasminogen activator inhibitor-1 phosphoinositide-3 kinase phenylmethanesulfonylfluoride . polyvinyl alcohol polyvinylidene fluoride red blood cell .rat sarcoma ribonucleic acid reactive oxygen species second(s) DNA synthesis phase/DNA replication phase SMAD anchor for receptor activation .. SMAD binding element sodium dodecyl sulphate standard error of the mean serine protease inhibitor E1 small body size protein Small phenotype and Mothers Against Decapentaplegic related protein smooth muscle cell .superoxide dismutase TGF- $\beta$ receptor I(I) Tris-buffered saline containing Tween-20 $\mathrm{N}, \mathrm{N}, \mathrm{N}^{\prime}, \mathrm{N}^{\prime}$-tetramethylethylenediamine transcription factor transforming growth factor beta .tris(hydroxymethyl)aminomethane TGF- $\beta$-stimulated clone 22 domain family 3 


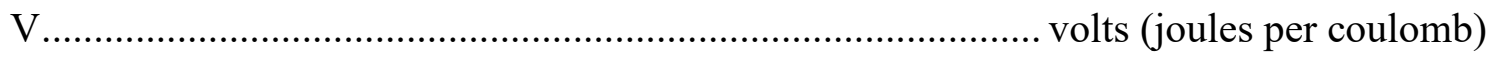

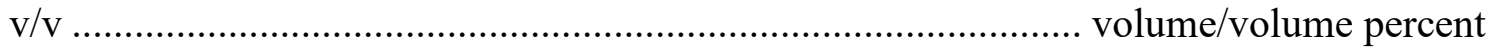

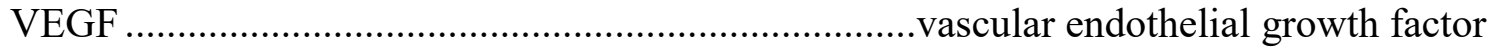

w/v ........................................................eight/volume percent (mass concentration) 


\section{LIST OF TABLES}

Table 1 - Immunoblotting conditions for experiments run on Trachemys scripta elegans liver tissues.

Table 2 - Immunoblotting conditions for experiments run on Trachemys scripta elegans white muscle tissues.

Table 3 - Immunoblotting conditions for experiments run on Trachemys scripta elegans red muscle tissues.

Table 4 - Immunoblotting conditions for experiments run on Trachemys scripta elegans kidney tissues.

Table 5 - Immunoblotting conditions for experiments run on Trachemys scripta elegans heart tissues.

Table 6 - Immunoblotting conditions for experiments run on Xenopus laevis liver tissues.

Table 7 - Immunoblotting conditions for experiments run on Xenopus laevis muscle tissues.

Table 8 - List of antibodies, their properties, and details.

Table 9 - Epitopes of specific antibodies used in this study. 


\section{LIST OF FIGURES}

Figure 1.1 - (A) Lactate shell buffering adaptation by freshwater turtles. (B)

Transitions to and from a hypometabolic state in the anoxia-tolerant turtle.

Figure 1.2 - Downregulation of the principal energetically demanding metabolic processes in Trachemys scripta elegans hepatocytes under normoxia and anoxia. A graphical (A) and tabular (B) representations of the suppression of cellular processes are shown.

Figure 1.3 - Graphs depicting the course of metabolic rate depression during anoxic submergence at $24^{\circ} \mathrm{C}$ (top) and $3^{\circ} \mathrm{C}$ (bottom) in the painted turtle (Chrysemys picta bellii).

Figure 1.4 - (A) Distributions of the red-eared slider (left) and the painted turtle (right) in the western hemisphere. (B) World distribution of the African clawed frog (Xenopus laevis).

Figure 1.5 - The TGF- $\beta$ signaling pathway.

Figure 2.1 - Effects of five hour and twenty hour anoxic exposure on the protein expression of total SMAD1 (52 kDa) and phospho-SMAD1/5 (serine 463/465) in red-eared slider liver as determined by immunoblotting.

Figure 2.2 - Effects of five hour and twenty hour anoxic exposure on the protein expression of total SMAD2 (52 kDa) and phospho-SMAD2 (serine 465/467) in red-eared slider liver as determined by immunoblotting.

Figure 2.3 - Effects of five hour and twenty hour anoxic exposure on the protein expression of total SMAD3 (48 kDa) and phospho-SMAD3 (serine 423/425) in red-eared slider liver as determined by immunoblotting. 
Figure 2.4 - Effects of five hour and twenty hour anoxic exposure on the protein expression of total SMAD4 $(60 \mathrm{kDa})$ in red-eared slider liver as determined by immunoblotting.

Figure 2.5 - Effects of five hour and twenty hour anoxic exposure on the protein expression of total SMAD5 (52 kDa) and phospho-SMAD1/5 (serine 463/465) in red-eared slider liver as determined by immunoblotting.

Figure 2.6 - Effects of five hour and twenty hour anoxic exposure on the protein expression of total SMAD1 (52 kDa) and phospho-SMAD1/5 (serine 463/465) in red-eared slider white muscle as determined by immunoblotting.

Figure 2.7 - Effects of five hour and twenty hour anoxic exposure on the protein expression of total SMAD2 (52 kDa) and phospho-SMAD2 (serine 465/467) in red-eared slider white muscle as determined by immunoblotting.

Figure 2.8 - Effects of five hour and twenty hour anoxic exposure on the protein expression of total SMAD3 (48 kDa) and phospho-SMAD3 (serine 423/425) in red-eared slider white muscle as determined by immunoblotting.

Figure 2.9 - Effects of five hour and twenty hour anoxic exposure on the protein expression of total SMAD4 $(60 \mathrm{kDa})$ in red-eared slider white muscle as determined by immunoblotting.

Figure 2.10 - Effects of five hour and twenty hour anoxic exposure on the protein expression of total SMAD5 (52 kDa) and phospho-SMAD1/5 (serine 463/465) in red-eared slider white muscle as determined by immunoblotting. 
Figure 2.11 - Effects of five hour and twenty hour anoxic exposure on the protein expression of total SMAD1 (52 kDa) and phospho-SMAD1/5 (serine 463/465) in red-eared slider red muscle as determined by immunoblotting.

Figure 2.12 - Effects of five hour and twenty hour anoxic exposure on the protein expression of total SMAD2 $(52 \mathrm{kDa})$ and phospho-SMAD2 (serine 465/467) in red-eared slider red muscle as determined by immunoblotting.

Figure 2.13 - Effects of five hour and twenty hour anoxic exposure on the protein expression of total SMAD3 (48 kDa) and phospho-SMAD3 (serine 423/425) in red-eared slider red muscle as determined by immunoblotting.

Figure 2.14 - Effects of five hour and twenty hour anoxic exposure on the protein expression of total SMAD4 $(60 \mathrm{kDa})$ in red-eared slider red muscle as determined by immunoblotting.

Figure 2.15 - Effects of five hour and twenty hour anoxic exposure on the protein expression of total SMAD5 (52 kDa) and phospho-SMAD1/5 (serine 463/465) in red-eared slider red muscle as determined by immunoblotting.

Figure 2.16 - Effects of five hour and twenty hour anoxic exposure on the protein expression of total SMAD1 (52 kDa) and phospho-SMAD1/5 (serine 463/465) in red-eared slider kidney as determined by immunoblotting.

Figure 2.17 - Effects of five hour and twenty hour anoxic exposure on the protein expression of total SMAD2 $(52 \mathrm{kDa})$ and phospho-SMAD2 (serine 465/467) in red-eared slider kidney as determined by immunoblotting. 
Figure 2.18 - Effects of five hour and twenty hour anoxic exposure on the protein expression of total SMAD3 (48 kDa) and phospho-SMAD3 (serine $423 / 425)$ in red-eared slider kidney as determined by immunoblotting.

Figure 2.19 - Effects of five hour and twenty hour anoxic exposure on the protein expression of total SMAD4 (60 kDa) in red-eared slider kidney as determined by immunoblotting.

Figure 2.20 - Effects of five hour and twenty hour anoxic exposure on the protein expression of total SMAD5 (52 kDa) and phospho-SMAD1/5 (serine 463/465) in red-eared slider kidney as determined by immunoblotting.

Figure 2.21 - Effects of five hour and twenty hour anoxic exposure on the protein expression of total SMAD1 (52 kDa) and phospho-SMAD1/5 (serine 463/465) in red-eared slider heart as determined by immunoblotting.

Figure 2.22 - Effects of five hour and twenty hour anoxic exposure on the protein expression of total SMAD2 (52 kDa) and phospho-SMAD2 (serine 465/467) in red-eared slider heart as determined by immunoblotting.

Figure 2.23 - Effects of five hour and twenty hour anoxic exposure on the protein expression of total SMAD3 (48 kDa) and phospho-SMAD3 (serine 423/425) in red-eared slider heart as determined by immunoblotting.

Figure 2.24 - Effects of five hour and twenty hour anoxic exposure on the protein expression of total SMAD4 (60 kDa) in red-eared slider heart as determined by immunoblotting. 
Figure 2.25 - Effects of five hour and twenty hour anoxic exposure on the protein expression of total SMAD5 (52 kDa) and phospho-SMAD1/5 (serine 463/465) in red-eared slider heart as determined by immunoblotting.

Figure 2.26 - Effects of five hour and twenty hour anoxic exposure on the protein expression of TGF- $\beta$ precursor $(45 \mathrm{kDa})$ in red-eared slider liver, white muscle, red muscle, kidney, and heart as determined by immunoblotting.

Figure 2.27 - Effects of five hour and twenty hour anoxic exposure on the protein expression of total c-Myc $(47 \mathrm{kDa})$ and phospho-c-Myc (threonine $58 /$ serine 62 ) in red-eared slider red muscle as determined by immunoblotting.

Figure 2.28 - The maturation of TGF- $\beta$ cytokine ligands.

Figure 2.29 - Transcription factors that regulate homeostasis of a differentiated cell along with their associated gene.

Figure 2.30 - The immense intricacy of the mTOR pathway and its connection to FoxO1, HIF-1, PI3K and Akt.

Figure 2.31 - Development of the vascular system.

Figure 2.32 - Role of SMADs in angiogenesis, heart failure, and cardiac development.

Figure 2.33 - TGF- $\beta$ signaling pathways in endothelial cells.

Figure 3.1 - Effects of medium and high dehydration on the protein expression of SMAD1 (52 kDa) and phospho-SMAD1/5 (serine 463/465) in African clawed frog liver as determined by immunoblotting. 
Figure 3.2 - Effects of medium and high dehydration on the protein expression of SMAD2 (52 kDa) and phospho-SMAD2 (serine 465/467) in African clawed frog liver as determined by immunoblotting.

Figure 3.3 - Effects of medium and high dehydration on the protein expression of SMAD3 (48 kDa) and phospho-SMAD3 (serine 423/425) in African clawed frog liver as determined by immunoblotting.

Figure 3.4 - Effects of medium and high dehydration on the protein expression of SMAD4 $(60 \mathrm{kDa})$ in African clawed frog liver as determined by immunoblotting.

Figure 3.5 - Effects of medium and high dehydration on the protein expression of SMAD5 (52 kDa) and phospho-SMAD1/5 (serine 463/465) in African clawed frog liver as determined by immunoblotting.

Figure 3.6 - Effects of medium and high dehydration on the protein expression of SMAD1 (52 kDa) and phospho-SMAD1/5 (serine 463/465) in African clawed frog muscle as determined by immunoblotting.

Figure 3.7 - Effects of medium and high dehydration on the protein expression of SMAD2 (52 kDa) and phospho-SMAD2 (serine 465/467) in African clawed frog muscle as determined by immunoblotting.

Figure 3.8 - Effects of medium and high dehydration on the protein expression of SMAD3 (48 kDa) and phospho-SMAD3 (serine 423/425) in African clawed frog muscle as determined by immunoblotting.

Figure 3.9 - Effects of medium and high dehydration on the protein expression of SMAD4 $(60 \mathrm{kDa})$ in African clawed frog muscle as determined by immunoblotting. 
Figure 3.10 - Effects of medium and high dehydration on the protein expression of SMAD5 (52 kDa) and phospho-SMAD1/5 (serine 463/465) in African clawed frog muscle as determined by immunoblotting.

Figure 3.11 - Effects of medium and high dehydration on the protein expression of TGF- $\beta$ precursor $(45 \mathrm{kDa})$ in African clawed frog liver and muscle as determined by immunoblotting.

Figure 3.12 - Effects of medium and high dehydration on the protein expression of total c-Myc (47 kDa) and phospho-c-Myc (threonine 58/serine 62) in African clawed frog liver as determined by immunoblotting.

Figure 3.13 - Effects medium and high dehydration on the protein expression of total c-Fos (40 kDa) and phospho-c-Fos (threonine 232) in the African clawed frog liver as determined by immunoblotting.

Figure 3.14 - Effects of medium and high dehydration on the protein expression of total c-Myc (47 kDa) and phospho-c-Myc (threonine 58/serine 62) in African clawed frog muscle as determined by immunoblotting.

Figure 3.15 - Effects medium and high dehydration on the protein expression of total c-Fos (40 kDa) and phospho-c-Fos (threonine 232) in the African clawed frog muscle as determined by immunoblotting.

Figure 3.16 - Gene regulation in TGF- $\beta$-induced cell cycle arrest.

Figure 3.17 - The sources and cellular responses to ROS.

Figure 3.18 - A c-Myc-dependent survival pathway activated by oxidative stress.

Figure 4.1 - Stages of torpor-arousal cycle. 
Figure 4.2 - The distribution of wood frog (Rana sylvatica) in the western hemisphere. 


\section{LIST OF APPENDICES}

APPENDIX A - Animal Specific Protein Sequences 168

APPENDIX B - Immunoblotting Conditions 174

APPENDIX C - List of Antibodies 182

APPENDIX D - List of Epitopes of Specific Antibodies 184

APPENDIX E - Ensuring Antibody Specificity and Band Selectivity for

$\begin{array}{ll}\text { Immunoblotting } & 186\end{array}$

APPENDIX F - Phylogenic Trees of SMAD Proteins 188 


\section{Chapter 1 General Introduction}

All around me are familiar faces

Worn out places, worn out faces

Bright and early for their daily races

Going nowhere, going nowhere 


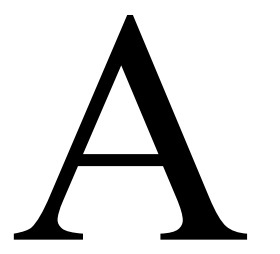

cross the globe's lands and oceans, there exists an enormous number of weather patterns, environmental conditions, topography, and flora. Not only do all these factors exist at any one time at any one location, but all of these characteristics vary with the seasons experienced here on Earth. The surface area of the Earth spans 510 million square kilometers - of which 149 million square kilometers are land. With such a massive landscape there will inevitably exist conditions which are more extreme than others and which may require special adaptations in order to withstand specific environmental condition(s). The fauna inhabiting a certain region will develop survival strategies and coping mechanisms to allow them to increase the population of their species, and/or develop evolutionary advantages over the rest of the organisms living within the same region.

Some organisms can tolerate extreme environmental stresses such as dehydration and anoxia (the complete lack of breathable oxygen either in air and/or dissolved in water). Trachemys scripta elegans, the red-eared slider turtle, is a well-known model organism used to study the effects of anoxia on cellular systems, whereas Xenopus laevis, the African clawed frog, is a model organism on studying the effects of whole body dehydration. Under these conditions, energy and fuel supply, particularly its conservation, becomes a main issue, along with waste by-product accumulation.

\section{1 - Strategic Stress Restraint under Anoxia}

The red-eared slider immerses itself in water during the winter months to escape predation and the sub-zero surface temperatures of the northern United States, mimicking the survival strategy of the painted turtle whose natural habitat is primarily in the northern United States, and southern Canada (Figure 1.4A). While immersed its access to gaseous 
oxygen is restricted and thus experiences long-term anoxia. Organisms that are intolerant of anoxic conditions typically face a rapidly degrading situation at the biochemical level; within minutes of oxygen deprivation their conditions can become as dire as permanent brain damage or even death. Oxygen is the basis for most metabolic energy production (ATP, adenosine triphosphate) in aerobic cells; as the final electron acceptor of the electron transport chain, it facilitates the proton pumps that in turn drive ATP synthesis via oxidative phosphorylation. Without ATP, all processes that require ATP energy begin to grind to a standstill. Active ion pumps (ATP-dependent) begin to fail while passive ion channels are not affected (ATP-independent); the result is that cells rapidly lose their membrane potential difference (Hochachka and Lutz, 2001), which leads to a breakdown in the ion gradients across all cell membranes. In brain, for example, cells then become flooded with calcium ions $\left(\mathrm{Ca}^{2+}\right)$ accompanied by a release in excitatory neurotransmitters. This rise in calcium ion concentration and neurotransmitters signals apoptosis (programmed cell death) and can quickly lead to death or other dangerous outcomes for the organism (Orrenius et al., 2003). Most animals have developed mechanisms to avoid these consequences when faced with hypoxia (low levels of available oxygen), and even short-term anoxia. Mechanisms that can counteract low oxygen stress include upgraded oxygen delivery to the tissues and an increase in anaerobic ATP production by glycolysis to compensate for the reduced ATP output from oxidative phosphorylation. Physiological responses of animals to low oxygen can include: increased ventilation and lung gas exchange, a greater level of oxygen unloading from circulating hemoglobin, and the release of red blood cells (RBC) from the spleen to effectively increase the total oxygen load capacity of an animal's circulatory system. Given that the turtle transitions into an anaerobic form of metabolism 
there would be a massive accumulation of lactic acid in the organism creating a condition whereby it would perish due to acidosis, however instead the turtle counteracts this consequence of glycolysis by using its shell to buffer its blood $\mathrm{pH}$ via $\mathrm{Ca}^{2+}$ ions, while the carbonate ions from the shell sequester the added protons (Ultsch and Jackson, 1982; Ultsch, 2006; Jackson, 2000; Jackson, 2002; Jackson et al., 2007) (Figure 1.1A). These biochemical and physiological adjustments are adequate to endure short-term hypoxia/anoxia, but long-term or severe oxygen deprivation has proven fatal to many species.

\section{2 - Desert Dehydration Determinations}

Most organisms are made up mostly of water by weight and volume. We can think of the ratio of water contained in the body to total body mass as a percentage, and also a value that can deviate only minimally in most animals - that is, most animals maintain their percentage body water within narrow limits and cannot withstand significant long-term dehydration. Studies have been done on many species to determine the effects of different dehydration levels all the way up to the effect of dehydration on human cognition and physical performance. Desiccation is known to affect membranes and cause severe damage to proteins (França et al., 2007). However, some species have extreme dehydration tolerances; animals such as brine shrimp and nematodes are capable of surviving when virtually all of their body water is removed, a phenomenon called anhydrobiosis. One can think of dehydration stress tolerance in two forms: (1) "drought tolerance" (medium dehydration) where the water content falls below about $23 \%$ (on a fresh weight basis) producing no bulk cytoplasmic water present amongst an organism's cells; (2) “desiccation tolerance" is when an organism is further dehydrated and the hydration shells surrounding 
its molecules is progressively lost (Hoekstra et al., 2001; França et al., 2007). Among terrestrial vertebrate animals, the greatest dehydration tolerances are found among amphibians. For example, as earlier mentioned, Xenopus laevis is capable of enduring a high level of dehydration - the loss of up to $35 \%$ of its total body water (Romspert, 1976). For many frog and toad species living in seasonally hot and arid environments, dehydration tolerance is key to survival and is an integral part of the phenomenon of estivation and $X$. laevis in no exception inhabiting the ponds of the otherwise seasonally dry areas of subSaharan Africa (Figure 1.4B). Estivation as a survival strategy has three main mechanisms: (i) metabolic rate suppression (i.e. descent into a hypometabolic state where cell energy demands are greatly reduced), (ii) nitrogen regulation, and (iii) water retention (Storey and Storey, 2012). Under normal conditions, $X$. laevis is virtually fully aquatic, living in ponds, marshes and streams but in the African summer, these bodies of water frequently dry up. The frogs have then been observed to dig into the mud where they can enter estivation; they can use the surrounding mud to help minimize their body water loss as well as raise their body osmolality to further limit water loss (Tinsley and Kobel, 1996; Balinsky et al., 1967). Under normal conditions in the aquatic environment, X. laevis excretes its nitrogenous wastes as ammonia, but when under desiccation stress the urea cycle is upregulated and suppresses the production of ammonia while increasing the synthesis of urea. Urea is retained by the frog's body and the elevated concentration of this osmolyte helps to retain body water in both plasma and cells (Balinsky, 1961; Balinsky et al., 1969). When frogs retreat into the moist mud of drying ponds they are also unable to hunt for food and so turn to the mobilization of stored fuels (e.g. lipids, glycogen, protein) as their main sources of energy. Hypometabolism is also crucial to ensure that fuels can last as long as 
possible, hopefully long enough for the frog to survive through dry season (Secor, 2005; Storey, 2015; Storey and Storey, 2012).

\section{3 - Hypometabolism}

Common under both stresses and adaptations for surviving anoxia and dehydration is the need to regulate energy balance of the organism. One can think of ATP as the energy currency of the body and glycogen (in the turtle) and fat stores (in the frog) as a fuel bank. At the molecular level both animals must ensure that their energy ledger stays in the black or else risk death before environmental conditions have once again become favorable for active life. Red-eared slider turtles can slow their metabolic rate to about $10 \%$ of their normal metabolic rate, while the metabolic rate of estivating frogs can drop to $17-30 \%$ of the normal resting rate (Secor, 2005; Jackson, 2002) (Figure 1.3). This is achieved in part by the suppression of metabolic enzyme activity and the cessation of non-essential cellular processes such as protein synthesis (mTOR pathway) (Figures 1.1B, 1.2A, 1.2B). Animalspecific strategies are further discussed in the respective chapters of this thesis.

\section{4 - TGF- $\beta$ Superfamily}

The TGF- $\beta$ signaling pathway uses SMAD proteins to transduce extracellular signals received by receptors embedded in the cell membrane into the nucleus where they can bind to DNA and stimulate the expression of selected genes (Itoh et al., 2000; Alliston et al., 2008; Shi and Massagué, 2003; Derynck and Feng, 1997; Wrighton et al., 2009) (Figure 1.5). The pathway controls the ability of cells to proliferate, to grow, or to induce apoptosis, and is part of the set of signaling molecules that trigger a stem cell and cause it to differentiate. It is these abilities that make the TGF- $\beta$ superfamily a physiologically important pathway (Wrighton et al., 2009). 
SMADs are a set of highly conserved proteins that derive their name from the SMA protein (small) found in nematodes (Caenorhabditis elegans) and the MAD protein (mothers against decapentaplegic) found in fruit flies (Drosophila melanogaster) - a portmanteau of the two protein names (Macias et al., 2015; Massagué et al., 2005). The family has eight members which are divided into three classes: receptor SMADs (RSMADs), common SMADs (co-SMADs), and inhibitory SMADs (I-SMADs). SMADs 1, $2,3,5$, and 8 constitute the first group, SMAD4 is the only co-SMAD, while SMAD6 and 7 constitute the last group (Massagué et al., 2005, Heldin, Miyazono, and ten Dijke, 1997; Macias et al., 2015; Derynck and Miyazono, 2008; Attisano and Wrana, 2002). SMAD proteins are $42 \mathrm{kDa}$ to $60 \mathrm{kDa}$ in relative molecular mass, and contain two regions of homology known as the Mad Homology 1 (MH1) and Mad Homology 2 (MH2) domains (Heldin, Miyazono, and ten Dijke, 1997; Macias et al., 2015; Derynck and Feng, 1997; Derynck and Miyazono, 2008). These globular domains are held together by a proline rich linker; the former is found at the N-terminal and is responsible for the protein's DNA binding function. DNA binding occurs through $\beta$-hairpin structures that are stabilized through a zinc atom $\left(\mathrm{Zn}^{2+}\right.$ ion coordinates with three cysteine residues and a histidine residue) and seeks a specific sequence of nucleotides known as the SMAD binding element (SBE). This sequence varies across the SMAD proteins with 5'-CAGAC-3' (bolded nucleotides are contacted by the protein) being responsible for the binding to DNA by SMAD3 (Arg74, Gln76, and Lys81 are the residues responsible for the interaction) (Macias et al., 2015; Massagué et al., 2005) whereas 5'-TGTGC-3' is the DNA sequence identified to interact with SMAD5 (Li et al., 2000; Macias et al., 2015). Due to an insert (codified as exon 3) in the vicinity of the $\beta$-hairpin in the MH1 domain of SMAD2, its structure is 
altered prohibiting it from binding to DNA directly (Massagué et al., 2005). When this insert is removed (SMAD2 $\triangle \mathrm{E} 3$ ), it has been shown that SMAD2 is able to bind to DNA like SMAD3 (Yagi et al., 1999; Massagué et al., 2005). A single SBE is not enough to bind a SMAD to DNA in vivo since a SMAD protein's affinity for a SBE is too low. Concatemers frequently require extra factors for effective DNA binding of the SMAD protein despite having up to four SBEs (Massagué et al., 2005; Macias et al., 2015). This can be alleviated by having many SBE repeats with the same sequence and allowing multiple MH1 domain-SBE interactions by the same SMAD complex (Seoane et al., 2004). The MH2 domain, however, is responsible for protein-protein interaction and cytoplasmic retention signals, and is found at the carboxyl terminal of the protein (Derynck and Feng, 1997; Derynck and Miyazono, 2008, Macias et al., 2015). The linker arm joining the two domains possesses post-translational modification sites including both phosphorylation and ubiquitination sites, and can signal for nuclear export of the SMAD protein (Macias et al., 2015; Kretschmar et al., 2003; Kamato et al., 2014; Alarcón et al., 2009).

As mentioned previously, SMADs are proteins that transduce the signals that are created when TGF- $\beta$ or other extracellular signaling agents such as bone morphogenic proteins (BMPs), activins, and nodals bind to their respective plasma membrane receptors (Derynck and Feng, 1997; Derynck and Miyazono, 2008, Macias et al., 2015). Once ligands have bound to the heteromeric receptor complex comprised of two type I transmembrane receptors $(\mathrm{T} \beta \mathrm{RI})$ and two type II transmembrane receptors (T $\beta R I I)$, these two sets of receptors are brought together and the constitutively active type II receptor phosphorylates the serine and threonine residues located in the GS domain on the type I receptor (Wrighton et al., 2009; Franzén, 1995, Huse et al., 2001; Attisano and Wrana, 2002). The GS domain 
is about 30 residues in length and so called due to it being rich in glycine and serine residues with ${ }^{185}$ TTSGSGSG $^{192}$ as its central amino acid sequence. The GS domain must be multiply phosphorylated with at least three, and perhaps four or five, of the serine and threonine residues undergoing phosphorylation to fully activate T $\beta R I$ (Franzén, 1995; Huse et al., 2001). Once TRRI is phosphorylated, its serine/threonine kinases become activated and subsequently commence phosphorylating downstream SMAD proteins; specifically this involves the serine residues found on the SSXS motif at the C-terminal tail of each RSMAD (Wrighton et al., 2009; Heldin et al., 1997; Derynck and Feng, 1997).

SMADs are self-inhibited by an interaction between the MH1 and MH2 domains and phosphorylation results in the disruption of this interaction and allows the formation of homodimeric and heterodimeric complexes with other activated R-SMADs in the cytoplasm, as well as its release from SARA (SMAD anchor for receptor activation) (Attisano and Wrana, 2002; Kretzschmer and Massagué, 1998; Macias-Silva et al., 1996). Inactive R-SMADs form a complex with SARA, a chaperone to SMAD2 and SMAD3, and are carried to the cytoplasmic kinase receptor, T $\beta$ RI. SARA accomplishes the chaperone role through control of the subcellular localization of SMADs and through interaction with the receptor complex (Wrighton et al., 2009; Attisano and Wrana, 2002; Massagué, 2000; Itoh et al., 2002). The newly activated R-SMAD complexes are carried into the cytoplasm by SARA, and are released as the dimer coordinates with the co-SMAD, SMAD4 (Attisano and Wrana, 2002; Itoh et al., 2002). Once bound to SMAD4, the now trimeric complex is escorted into the nucleus. BMP ligands work in the same way as TGF- $\beta$ I-III. They activate analogous BMP kinase receptors I and II (BMPRI and BMPRII, respectively) that phosphorylate SMADs 1, 5, and 8 which become activated upon phosphorylation and are 
released from the receptor into the cytoplasm. A notable difference in BMP signaling is the existence of seven BMPRI which are referred to as activin receptor-like kinases (ALK) 1-7 (Wrighton et al., 2009). This set of R-SMAD proteins do not interact with SARA, but similarly form homo- and heterodimers which bind to SMAD4 forming a trimeric complex that is then shuttled to the nucleus. It should be mentioned that TGF- $\beta$ cytokines can stimulate SMAD1 phosphorylation without the presences of BMP cytokines or even a BMP kinase type I receptor (Wrighton et al., 2009). Upon entry into the nucleus, in both cases, the complex binds to p-300-CBP (CREB-binding protein) where the binding energy is lowered such that a third party transcription factor, such as AP-1, RUNX or FOXO can latch onto DNA and facilitate the transcription of various genes (Liberati et al. 1999; Fei et al., 2010). The genes that are targeted by the TGF- $\beta /$ SMAD signal transduction pathway depends heavily on the contextual determinants within the cell and more specifically in the nucleus. These determinants are most often specific to a cell lineage, differentiation stage, or metabolic condition. The signaling pathway plays a role as a context-dependent multifunctional signal device for regulation of the cell from its conception to its death (Macias et al., 2015).

\section{5 - Objectives and Hypotheses}

The focus of this thesis is to add upon fundamental truths about the mechanisms of anoxia or dehydration tolerance identified through previous research on the two model animals - Trachemys scripta elegans and Xenopus laevis. The biochemical and physiological responses that support the theory of metabolic rate depression being triggered by anoxia stress in the red-eared slider turtle, and through desiccation stress in the African clawed frog are usually linked and can be complex to elucidate as a whole. This study looks 
at the multifunctional transforming growth factor beta superfamily (TGF- $\beta$ ) and how it affects processes such as the cell cycle and autophagy in an attempt to allow tissues to maintain their function while simultaneously minimizing the energy costs associated with the tissue type. Five tissues (liver, white skeletal muscle, red muscle, kidney, heart) of adult red-eared slider turtles were analyzed for SMAD family member protein expressions, as well as expressions of those proteins that are upstream or downstream of the TGF- $\beta$ signaling pathway, whereas the same proteins were analyzed in two tissues from the African clawed frog (liver, muscle).

\subsection{1 - Objective 1: TGF- $\beta$ pathway, the Mediator for Cellular Metabolic Rate}

\section{Depression}

In response to anoxia in facultative anaerobes, cells will downregulate processes with high energy turnovers, and upregulate energetically efficient ATP-producing pathways in terms of ATP per mole of fuel used (Hochachka et al., 1996). Since the cell cycle is known to be a large consumer of ATP, there must be effectors upstream to signal an interruption of cell division under oxygen-limited conditions. The necessary signals may be coordinated via the TGF- $\beta$ pathway. Chapter 2 will explore these ideas.

Hypothesis I - Under oxygen-limited, low energy conditions in turtles, differential expression of TGF- $\beta$ cytokines, SMAD proteins and the cell cycle inhibitor proteins, $\mathrm{p} 21$ and p27 should occur, potentially halting tissue cell division and growth.

1.5.2-Objective 2: Maintaining Homeostasis in Tissues Through the TGF- $\beta$

Superfamily

Downregulation of every pathway is not necessarily favourable or required for oxygenlimited animal such as the submerged turtle. Heart function must continue and some 
swimming powered by skeletal muscle activity is still needed. Chapter 2 examines the differential expression of SMADs in the context of muscle atrophy, as well as downstream factors of SMADs that control homeostasis, cell adhesion, migration, and proliferation (Massagué, 2012). The kidneys and the heart are organs that would be required to be functional immediately following an anoxic exposure and therefore expected to be maintained via SMADs 1 and 5. BMPs have been shown to play a role in heart and kidney tissue development, and would be expected to play a major role in determining the regulation of processes occurring within these two tissues because they activate SMADs 1 and 5 as part of their signaling mechanism (Chuva de Sousa et al., 2004; Euler-Taimor and Heger, 2006).

Hypothesis II - Downregulation of SMAD transcription factors is expected in order to inhibit muscle growth and promote muscle atrophy in cardiac and red muscles. Kidney is expected to show an upregulation of SMAD family member proteins since SMAD1 has been implicated in renal development.

\section{$\underline{1.5 .3-O b j e c t i v e ~ 3: ~ T G F-~} \beta$ as an Important Liaison in Dehydration Survival}

Under desiccation stress, Xenopus laevis seek to reduce its energy consumption while also maintaining tight osmoregulation of its cells (Romspert, 1976, Seiter et al., 1978). Chapter 3 examines the effects of dehydration upon the TGF- $\beta$ superfamily and all its downstream transcription factors that control critical cell processes. The studies aim to determine if the TGF- $\beta$ superfamily plays an important role as an intermediate signaling pathway as an organism endures increasing desiccation stress over time. 
Hypothesis III - To aid dehydration survival, SMAD family member proteins are globally downregulated in liver tissues, while fewer and less dramatic changes occur in the muscle tissues of the frog. ROS can rise as a result of dehydration, SMAD protein expression should increase the phosphorylation of c-Myc. 


\section{6 - General Introduction Figures}

A)

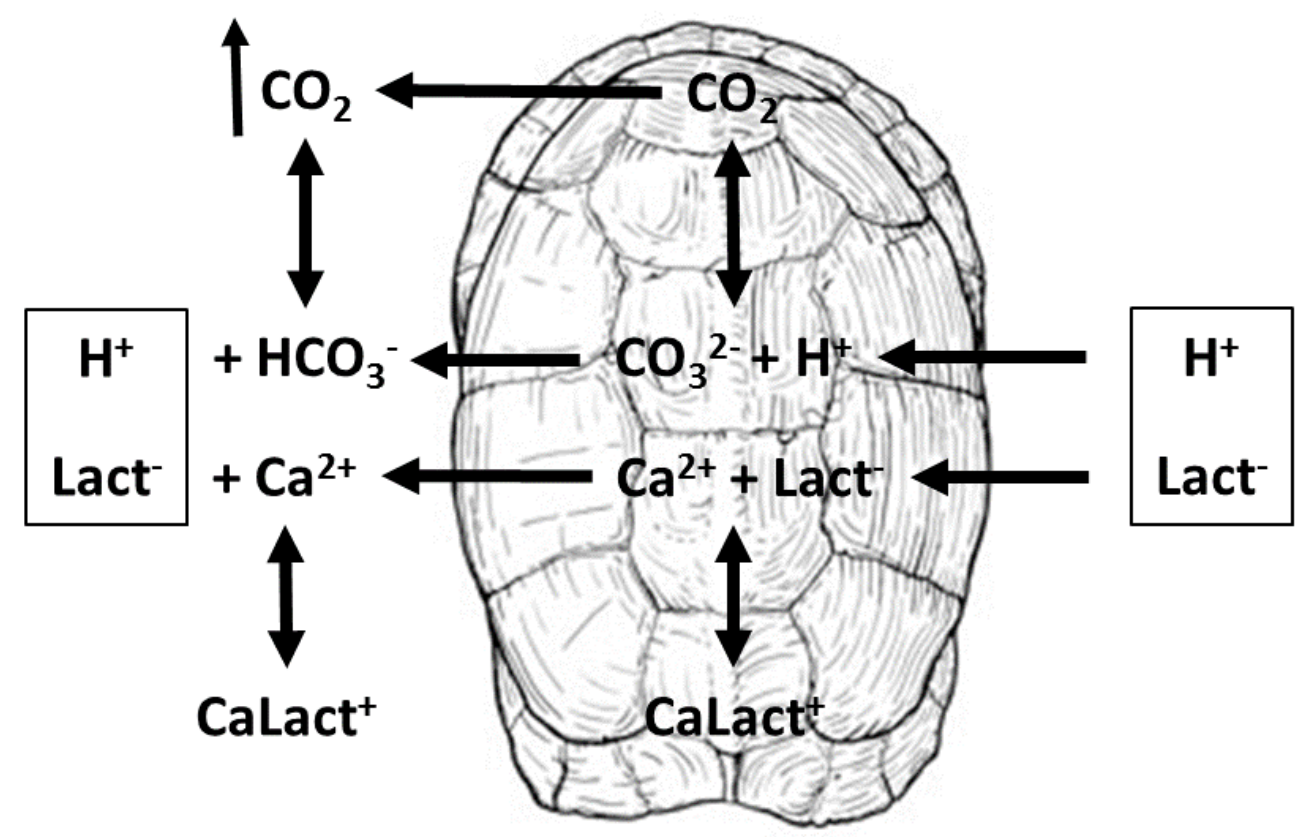

B)

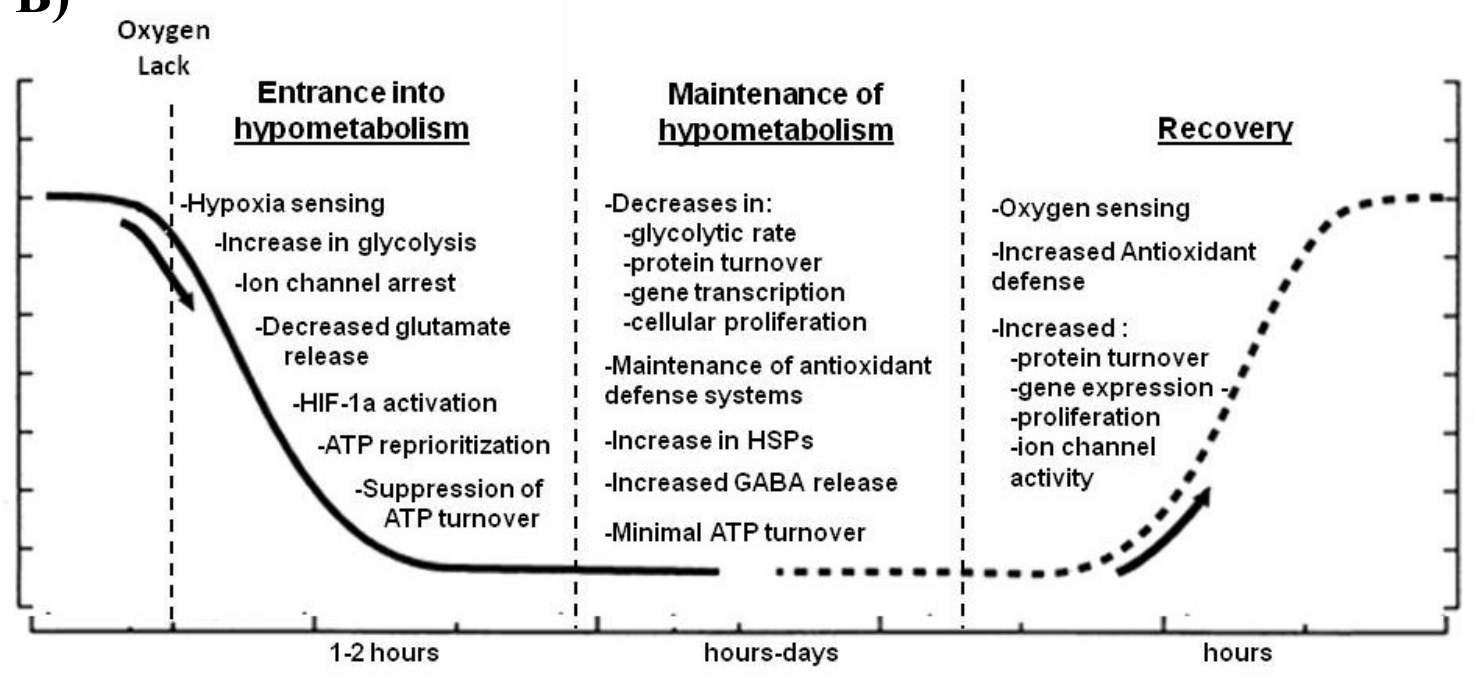

Figure 1.1 - (A) Lactate shell buffering adaptation by freshwater turtles to prevent acidosis over the course of the anoxic episode; adapted from Jackson (2000). (B) Transitions to and from a hypometabolic state in the anoxia-tolerant turtle triggering processes to endure oxygen deprivation and protection from oxidative damage upon oxygen reperfusion; reproduced from Biggar (2011). 
A)

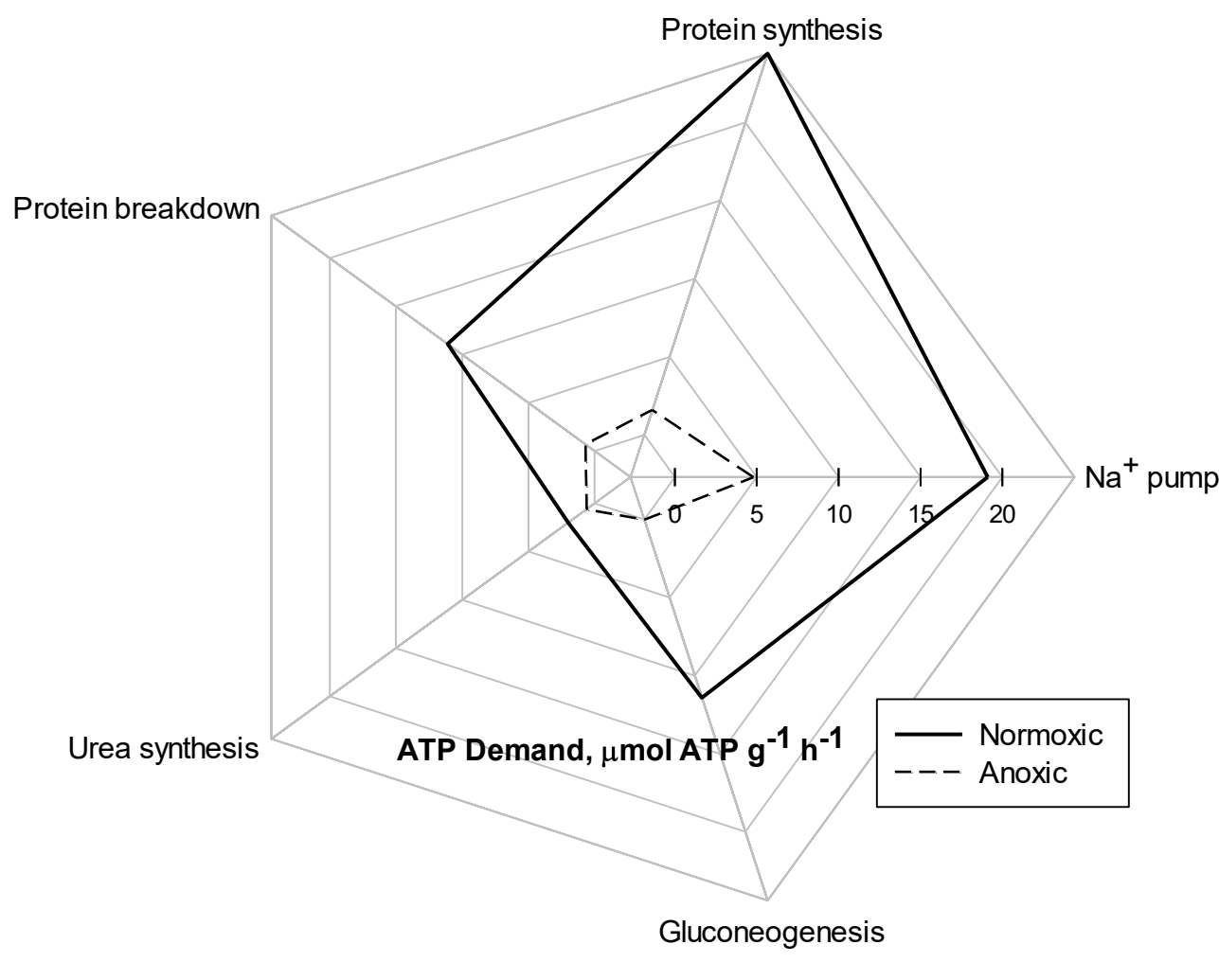

B)

ATP demand, $\mu$ mol ATP $\times \mathrm{g}^{-1} \times \mathrm{h}^{-1}$

\begin{tabular}{lccc}
\multicolumn{1}{c}{ Pathway } & Normoxia & Anoxia & \% suppression \\
\hline Total & 67.0 & 6.3 & 94 \\
$\mathrm{Na}^{+}$pump & 19.1 & 4.8 & 75 \\
Protein synthesis & 24.4 & 1.6 & 93 \\
Protein breakdown & 11.1 & 0.7 & 94 \\
Urea synthesis & 2.0 & 0.6 & 70 \\
Gluconeogenesis & 11.4 & 0.0 & 100 \\
\hline
\end{tabular}

Figure 1.2 - Downregulation of the principal energetically demanding metabolic processes in Trachemys scripta elegans hepatocytes under normoxia and anoxia. A graphical (A) and tabular (B) representations of the suppression of cellular processes are shown. The suppression of gluconeogenesis, urea synthesis, protein synthesis, protein breakdown and ion-motive ATP pumps results in a more than $90 \%$ decrease in ATP-consumption under anoxia. Table from Hochachka et al. (1996). 


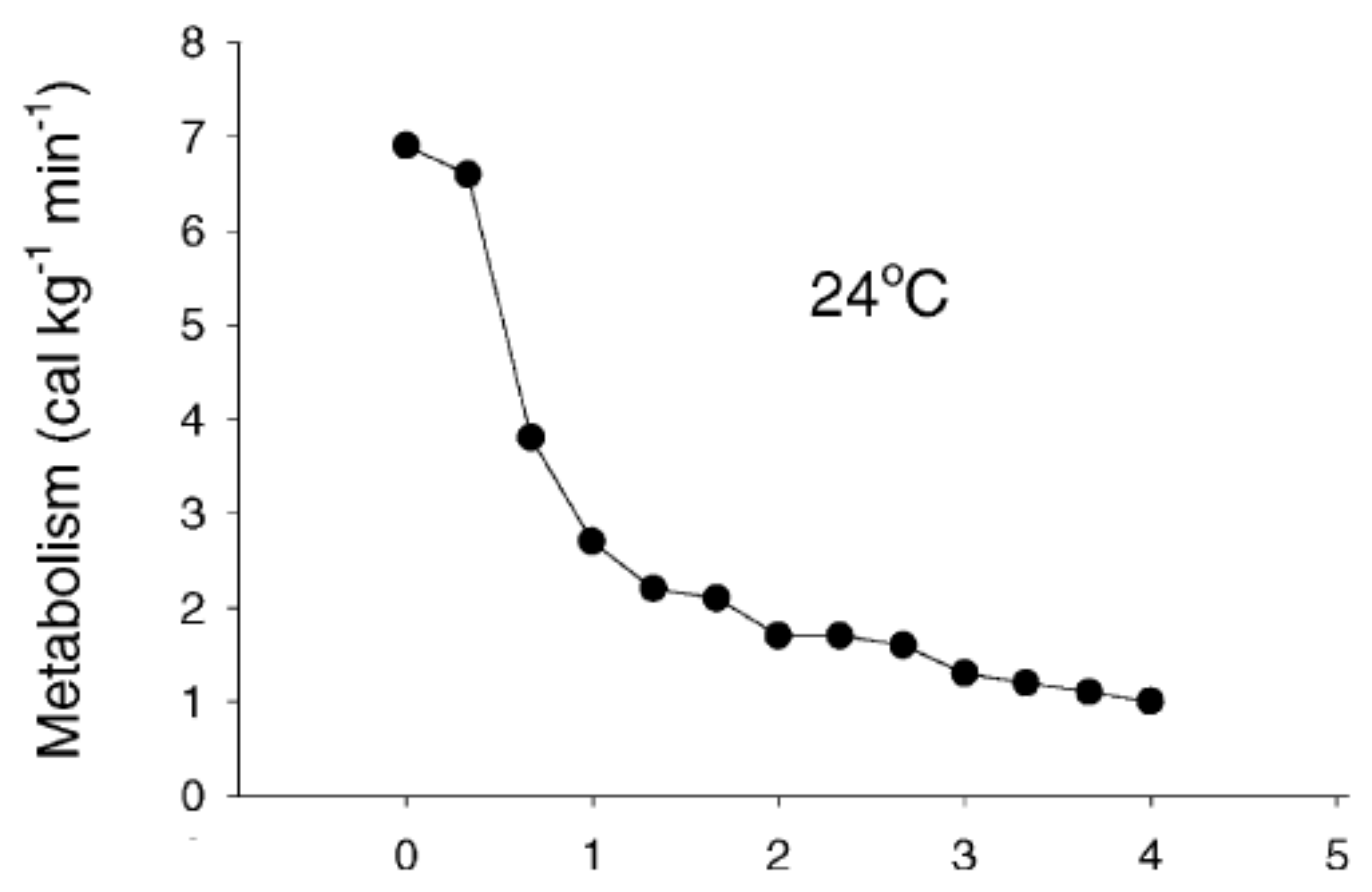

Hours of Anoxic Submergence

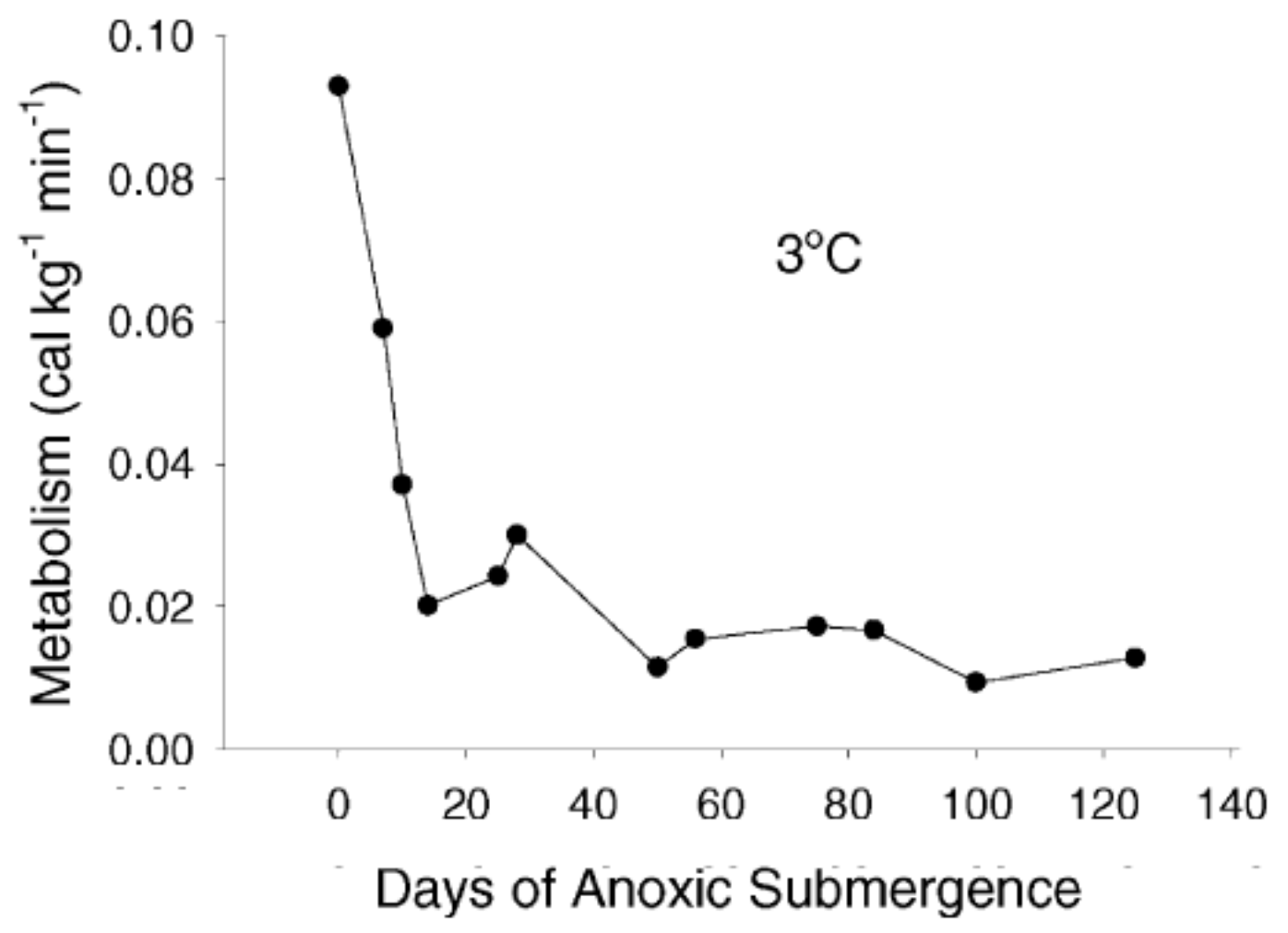

Figure 1.3 - Graphs depicting the course of metabolic rate depression during anoxic submergence at $24^{\circ} \mathrm{C}$ (top) and $3{ }^{\circ} \mathrm{C}$ (bottom) in the painted turtle (Chrysemys picta bellii), reproduced from Jackson (2002). 
A)
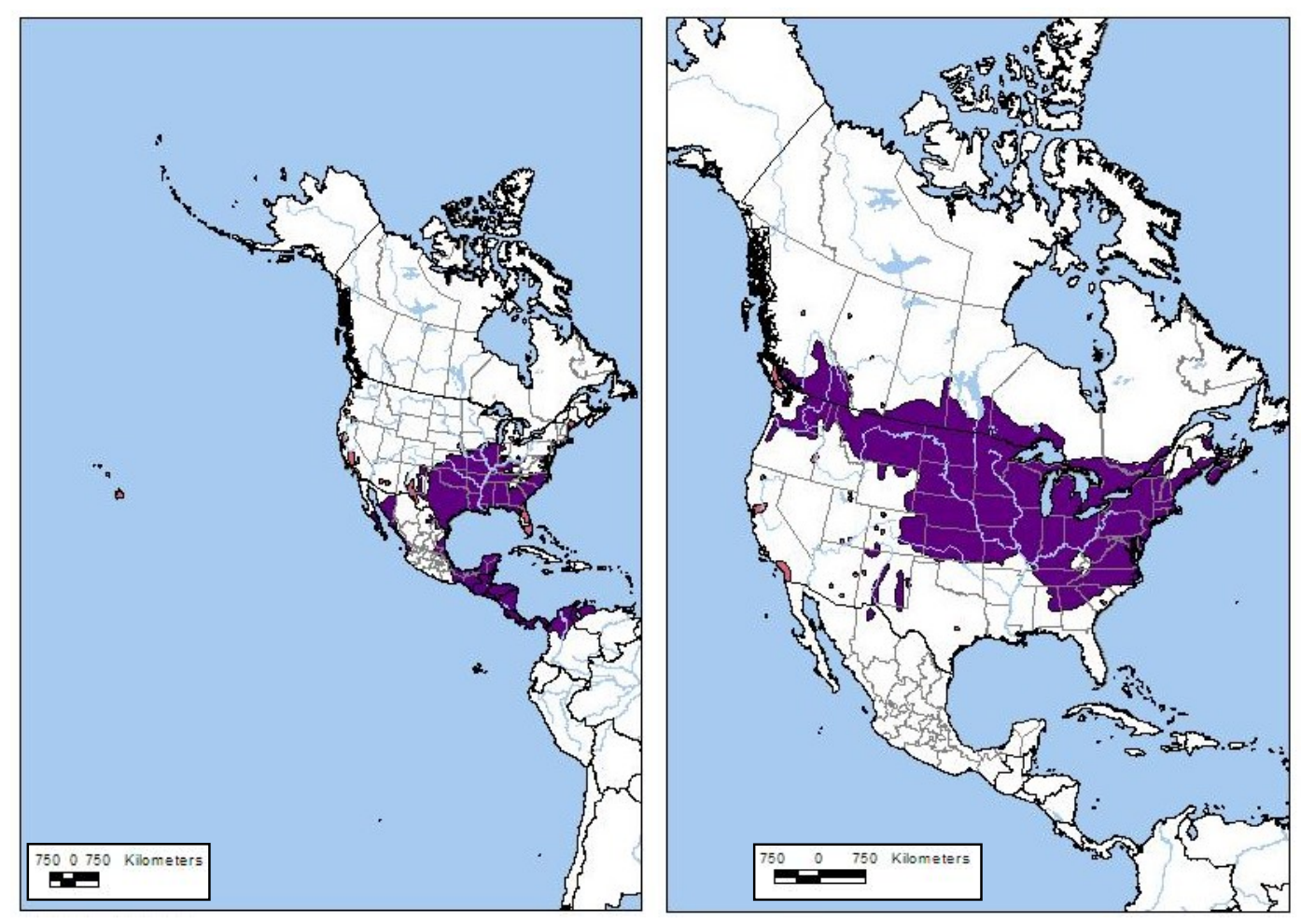

B)

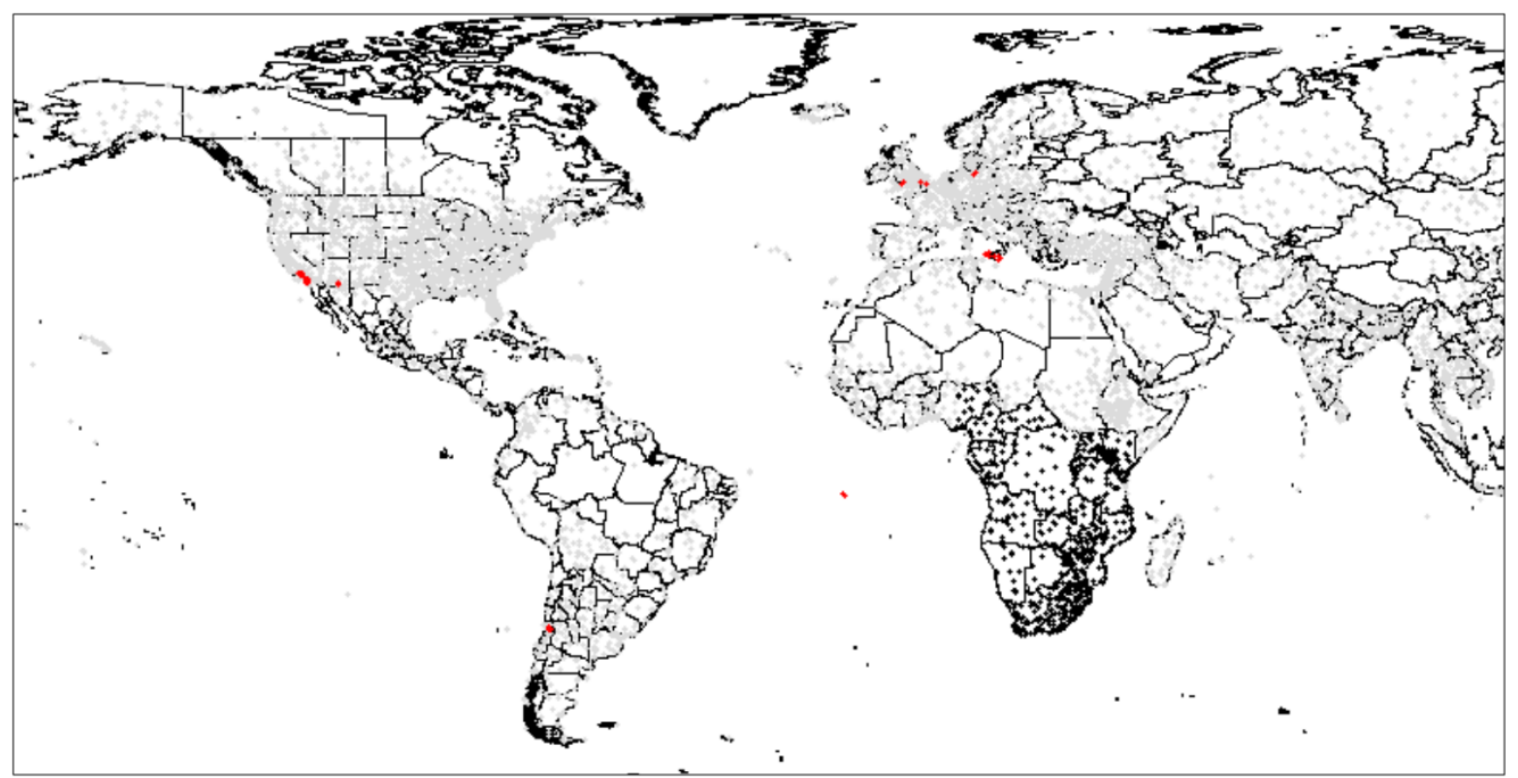

Figure 1.4 - (A) Distributions of the red-eared slider (left) and the painted turtle (right) in the western hemisphere (Montana Field Guide, 2008). (B) World distribution of the African clawed frog (Xenopus laevis); black dots depict natural populations while red dots depict introduced populations (Dept. Agri. Food, Western Australia). 


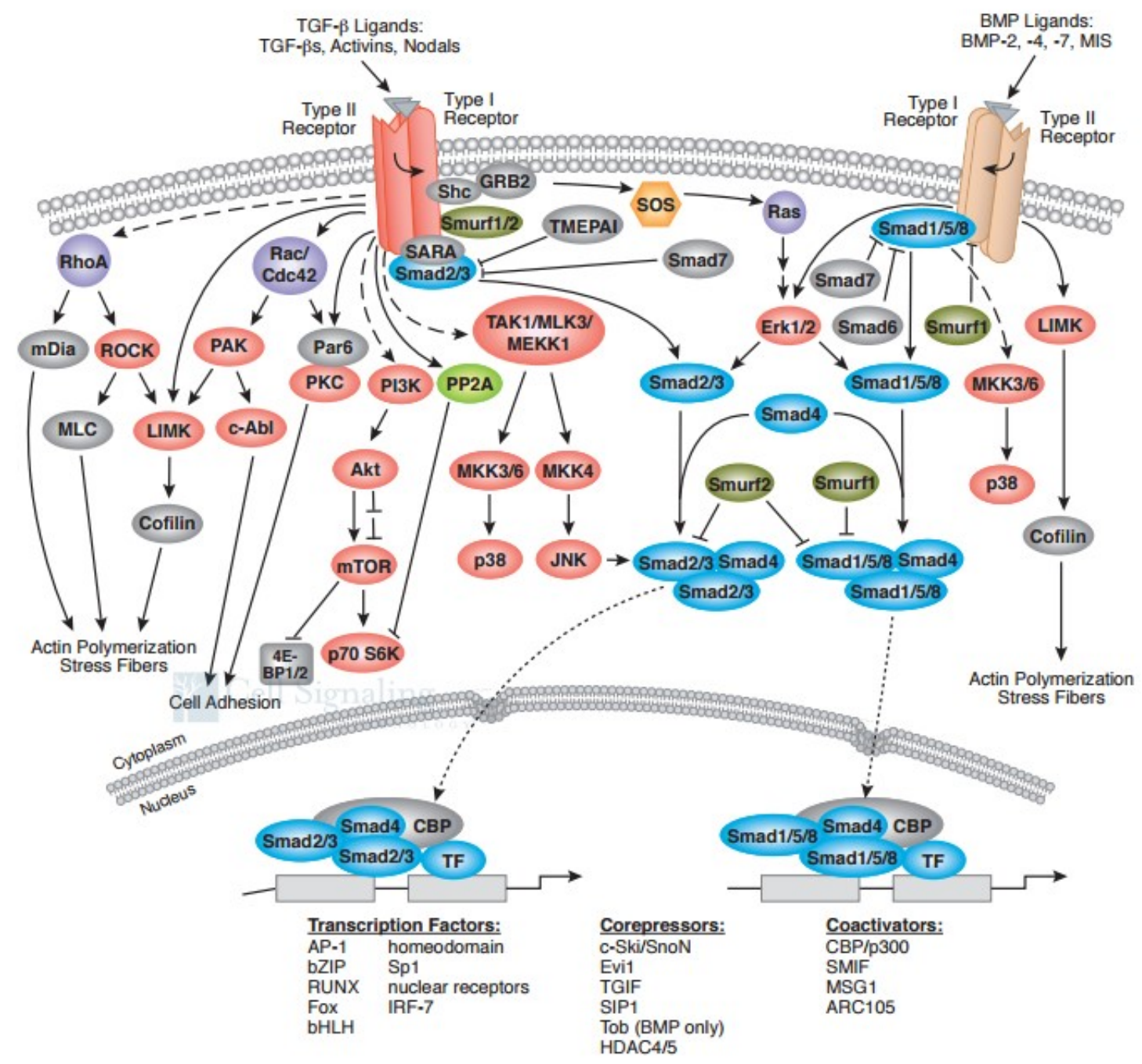

Figure 1.5 - The TGF- $\beta$ signaling pathway. TGF- $\beta$ and BMP cytokines bind to their respective transmembrane kinase receptors. Consequently, the type II receptor phosphorylates the type I receptor at the GS domain. R-SMADs coordinate with the type I receptor through SARA (SMAD2/3), or without SARA (SMAD1/5/8) and become phosphorylated at their SSXS motifs at the COOH tail. The co-SMAD, SMAD4, completes the trimeric complex and shuttles it to the nucleus where it binds to DNA in combination with other transcription factors to impart transcriptional control to the cell. Figure obtained from Cell Signaling (2014), www.cellsignal.com). 
Chapter 2

Effects of Anoxic Exposure on SMAD and its Downstream Protein Expression in Trachemys scripta elegans

Their tears are filling up their glasses

No expression, no expression

Hide my head, I wanna drown my sorrow

No tomorrow, no tomorrow 


\section{1 - Introduction}

The semiaquatic Trachemys scripta elegans spends the winter months underwater to avoid cold ambient air temperatures (often below $0^{\circ} \mathrm{C}$ ) and limited food availability in many parts of its range. Indeed, a number of turtle species do this and in some cases these lung-breathing vertebrates are forced to go without breathing air for long periods of time, sometimes in a "voluntary" way by resting underwater in a torpor-like state over the winter months and sometimes because they are unable to surface and breathe air due to ice covering the surface of lakes and ponds. For this reason and also because their lifestyle involves much breath-hold diving (to find food or avoid predators), various species of turtles have developed other modes of acquiring oxygen, namely through gas exchange across their buccal or cloacal epithelia, whereas other species have evolved an ability to endure oxygen deprivation for extremely long periods of time. Indeed, freshwater turtles such as red-eared sliders (T. s. elegans) and painted turtles (Chrysemys picta) are known as the champion facultative anaerobes among vertebrate animals. Both can survive under water without breathing for up to two weeks at $16-18^{\circ} \mathrm{C}$ and between 12 to 18 weeks at $3^{\circ} \mathrm{C}$ (Ultsch, 2006; Jackson, 2002) (Figure 1.3). Both species are models for many studies of the physiological and biochemical adaptations for anoxia survival.

\section{$\underline{2.1 .1-\text { Annual Anoxia Anomaly }}$}

Many studies have examined various physiological and biochemical adaptations that allow turtles to live without oxygen for long periods of time (Ultsch, 1985; Ultsch and Jackson, 1982; Herbert and Jackson, 1985; Gatten, 1987). For short-term oxygen limitation (i.e. diving for food or escaping predation), energy production from glycolysis alone may be sufficient to sustain energy demands (Clark and Miller, 1973; Jackson, 1968; Storey and 
Storey, 2004b). In the case of long-term oxygen restriction, such as when turtles overwinter underwater to avoid subzero temperatures, some additional strategies can apply. Some species of freshwater turtles can use extrapulmonary strategies to take up oxygen, particularly in cold water where oxygen content is high. Soft-shelled turtles possess the ability to exchange respiratory gases underwater across heavily vascularized epithelium linings of their throats and/or cloaca (Reese et al., 2003; Ultsch, 1985; Storey and Storey, 2004) allowing them to breathe without using their lungs. A strong suppression of metabolic rate (known as hypometabolism) triggered by submersion also reduces the metabolic demands of turtles. This can allow various species to survive for several months underwater (without oxygen) using perfected mechanisms of facultative anaerobiosis.

Among freshwater turtles, members of the genera Trachemys (pond slider turtles) and Chrysemys (painted turtles) stand paramount as the best facultative anaerobes. As mentioned previously, many animals including humans are sensitive to anoxia and can perish after only a few minutes without oxygen. These turtles as well as other anoxia tolerant organisms use five main coping strategies to survive:

1. By altering its own physiology and biochemistry in response to low oxygen as well as transitioning into an anoxic state at the cellular level. This includes processes for hypoxia sensing and signal transduction, metabolic reorganization, the regulation of neurotransmitters and ion channels.

2. Strongly suppressing both energy production and energy use by metabolic activities in a coordinated fashion to create an energy neutral environment in terms of ATP consumption and ATP production. The metabolic rate of the turtles has been measured to be just $10-20 \%$ of the normoxic resting rate at the same temperature 
(Jackson, 1968; Herbert and Jackson, 1985). This avoids the concerns associated with energy failure during hypometabolism while stretching body fuel reserves to last 5-10 times longer than they would if turtle wintered under aerobic conditions on land.

3. Diminishing the effects of oxidative stress from oxygen reperfusion when the organism must inevitably return to normoxic conditions.

4. Allowing signaling pathways to act as a barometer of low oxygen stress and permit swift cellular adjustments and mechanisms of protection as to promote survival.

5. Storing large amounts of glycogen in tissues to provide an anaerobic fuel source and tolerating the build-up of huge amounts of lactate, the end product of glycolysis.

Anoxia-tolerant turtles survive long term oxygen deprivation conditions that would in most cases cause permanent damage or be fatal for most other organisms. Anoxia is a condition where an organism has no oxygen, whereas hypoxia refers to low oxygen conditions below the threshold where the organism can meet normal oxygen requirements for its tissues. In this study, red-eared slider turtles were exposed to anoxic submergence ( $5 \mathrm{~h}$ and $20 \mathrm{~h}$ ) in deoxygenated water and then organ-specific changes in the expression pattern of multiple signaling proteins were assessed relative to control (normoxic) conditions.

When oxygen levels decline in the blood of submerged sliders several initial physiological responses are unleashed that attempt to increase oxygen delivery to tissues. The first are responses to hypoxia (low oxygen) that are common to most animals and include an increase in heart rates, an increase in hemoglobin unloading of oxygen in tissues, and a release of stored red blood cells from the spleen. These changes serve to maximize 
the amount of oxygen available to the turtle, its carrying capacity and its delivery to organs (Storey, 2004). However, below a critical point, a continued dwindling of oxygen supply and/or transition into full anoxia, requires a different strategy when mitochondrial ATP generation can no longer meet the demands for ATP production. When this happens, turtles turn to anaerobic methods of ATP production by elevating its glycolytic rate and by the consumption of creatine phosphate stores (although the phosphagen is mainly present only in muscle tissues). This is supported by huge reserves of glycogen stored in turtle liver. The anaerobic metabolism of glycogen is fairly inefficient, producing just three equivalents of ATP for every glucose equivalent cleaved off glycogen, whereas aerobic catabolism of the same amount of glucose results in $32 \mathrm{ATP}$ when the sugar is fully oxidized to $\mathrm{CO}_{2}$ and $\mathrm{H}_{2} \mathrm{O}$. Using glycolysis, glucose is converted into the end product lactic acid and this leads to acidosis if anoxia is prolonged. However, turtles have a solution to this problem; they release calcium carbonate from their shell using it to counter act the $\mathrm{H}^{+}$and lactate anion produced by glycolysis. Lactate is also stored into the shell thereby lowering its concentration in body fluids (Jackson, 2000; Figure 1.1A). Another survival strategy that helps the turtle cope with long-term anoxic conditions is the minimization of end-product toxicity by buffering the $\mathrm{pH}$ (namely in the blood) and the formation of oxygen radicals within the organism to effectively reduce the potential of damage through oxidative stress during recovery.

\subsection{2 - Metabolic Rate Depression}

To offset the limited options for ATP synthesis under anoxia (i.e. inefficient ATP production by glycolysis, consumption of phosphagen), turtles slow down their consumption of energy via metabolic rate depression by turning off non-essential 
"services". In addition to this metabolic rate decrease, animals also need to rebalance the rates of ATP-utilizing and ATP-generating processes and reprioritize the ATP usage by multiple kinds of metabolic processes so that overall the turtle can greatly extend the time that it can survive in the absence of oxygen in its environment. Such metabolic rate depression can also be observed in many organisms that must survive extreme environmental stresses. By using this survival strategy, the turtle may only need to consume energy at 10 to $20 \%$ its normal rate. The "services" that are suppressed to contribute to metabolic rate depression include reduced voluntary movement by skeletal muscles, reducing heart, breathing and kidney filtration rates, energy savings associated with slowed or halted feeding, digestion and absorption of nutrients as well as suppression of many energy-expensive metabolic processes including transcription, translation, transmembrane ion pumping, and cell division (Krivoruchko, 2010).

\section{2 - Methods and Materials}

\section{$\underline{2.2 .1-\text { Animal Treatments }}$}

Adult female red-eared sliders (Trachemys scripta elegans), 700-1500 g, were acquired from local suppliers and held at $5 \pm 1{ }^{\circ} \mathrm{C}$ in large plastic tubs (two turtles per tub) filled with dechlorinated tap water for several days before use. Control T. s. elegans turtles (CTSE) were sampled from this condition. For anoxia exposure, turtles were transferred to large buckets at $5 \pm 1{ }^{\circ} \mathrm{C}$ that had been previously bubbled with $\mathrm{N}_{2}$ gas for $1 \mathrm{~h} ; 2-3$ turtles were added per bucket in 30 minute intervals. Bubbling was continued for one hour after the last turtle was added and was reinitiated again during sampling of the animals. A wire mesh was fitted into the tank about $5 \mathrm{~cm}$ below the water surface so that turtles remained submerged throughout the 5 or $20 \mathrm{~h}$ experimental anoxic submergence $(5 \mathrm{hA}$ or $20 \mathrm{hA}$, 
respectively). All animals were killed by decapitation and then liver, kidney, heart, red muscle, and white skeletal muscle were rapidly dissected out, frozen in liquid nitrogen and stored at $-80^{\circ} \mathrm{C}$ until use. All animals were cared for in accordance with the guidelines of the Canadian Council on Animal Care, and all experimental procedures had the prior approval of the Carleton University Animal Care Committee.

\subsection{2 - Homogenization of Animal Tissues and Total Protein Isolation}

Total protein was extracted from liver, kidney, heart, red muscle, and white skeletal muscle samples from control, 5 hour and 20 hour anoxia treated turtles. Frozen tissues (approximately $0.5 \mathrm{~g}$ ) were crushed under liquid nitrogen and homogenized in $1 \mathrm{~mL}$ of homogenization buffer [20 mM 4-(2-hydroxyethyl)-1-piperazineethanesulfonic acid (HEPES) $\mathrm{pH}$ 7.5, $200 \mathrm{mM} \mathrm{NaCl}, 0.1 \mathrm{mM}$ ethylenediamine tetraacetate (EDTA), $10 \mathrm{mM}$ sodium fluoride $(\mathrm{NaF}), 1 \mathrm{mM}$ sodium orthovanadate $\left(\mathrm{Na}_{3} \mathrm{VO}_{4}\right), 10 \mathrm{mM} \quad \beta$ glycerophosphate). A few crystals of phenylmethylsulfonyl fluoride (PMSF) and $1 \mu \mathrm{L}$ of protease inhibitor cocktail $\quad[104 \mathrm{mM}$ 4-(2-aminoethyl)-benzenesulfonylfluoride hydrochloride (AEBSF), $80 \mu \mathrm{M}$ aprotinin, $4 \mathrm{mM}$ bestatin, $1.4 \mathrm{mM} \mathrm{E-64,} 2 \mathrm{mM}$ leupeptin, $1.5 \mathrm{mM}$ pepstatin] (Sigma-Aldrich, Oakville, ON) were added immediately before homogenization. The samples were centrifuged at $10,000 \times g$ for 15 minutes at $4{ }^{\circ} \mathrm{C}$ and the supernatant was collected.

Soluble protein concentration was determined using the Bio-Rad Protein Assay (5000006; Bio-Rad Laboratories (Canada) Ltd., Mississauga, ON, Canada) using bovine serum albumen (BSA) as the standard. The assay was carried out as follows: one volume of stock protein assay concentrate was diluted with four volumes of distilled deionized water $\left(\mathrm{ddH}_{2} \mathrm{O}\right)$ to make a working Bio-Rad protein reagent. Into each non-binding microplate 
well, $10 \mu \mathrm{L}$ of the protein sample was added along with $190 \mu \mathrm{L}$ of the working Bio-Rad reagent. This assay was done in triplicate to ensure accurate protein concentrations. The well contents were allowed to react for five minutes, before the microplate was inserted into a BioTek Powerwave HT (Cat no.: RPRWI; Norgen Biotek Corp., Thorold, ON, Canada) and read using the associated BioTek Gen5 ${ }^{\mathrm{TM}}$ data analysis software at a wavelength of $595 \mathrm{~nm}$.

Sample concentrations were then adjusted to $10 \mu \mathrm{g} / \mu \mathrm{L}$ by addition of small volumes of homogenization buffer. Samples were then mixed 1:1 v:v with SDS loading buffer (100 $\mathrm{mM}$ Tris(hydroxymethyl)aminomethane (Tris) base, $4 \% \mathrm{w} / \mathrm{v}$ SDS, $20 \% \mathrm{v} / \mathrm{v}$ glycerol, $0.2 \%$ $\mathrm{w} / \mathrm{v}$ bromophenol blue, $10 \% \mathrm{v} / \mathrm{v} 2$-mercapthoethanol) for a final protein concentration of $5 \mu \mathrm{g} / \mu \mathrm{L}$. Samples were boiled for five minutes to denature the proteins, immediately cooled on ice and stored at $-40^{\circ} \mathrm{C}$ until use.

\subsection{3 - SDS Polyacrylamide Gel Electrophoresis and Immunoblotting}

Discontinuous, 15-well SDS-polyacrylamide gels (10\%-15\% v/v acrylamide/bisacrylamide, $400 \mathrm{mM}$ Tris base $\mathrm{pH} 8.8,0.1 \% \mathrm{w} / \mathrm{v}$ sodium dodecyl sulfate (SDS), $0.1 \% \mathrm{w} / \mathrm{v}$ ammonium persulfate (APS), $\quad 0.04 \% \quad \mathrm{v} / \mathrm{v} \quad \mathrm{N}, \mathrm{N}, \mathrm{N}^{\prime}, \mathrm{N}^{\prime}$-tetramethylethylenediamine (TEMED) of a $1.00 \mathrm{~mm}$ thickness, Bio-Rad Laboratories (Canada), Mississauga, ON, Canada ) were prepared with $5 \%$ upper, stacking gels $(5 \% \mathrm{v} / \mathrm{v}$ acrylamide/bis-acrylamide, $130 \mathrm{mM}$ Tris base $\mathrm{pH}$ 6.8, 0.1\% w/v SDS, 0.1\% w/v APS, 0.1\% v/v TEMED). For each gel, an equal amount of soluble protein $(20-30 \mu \mathrm{g})$ was loaded into each well and proteins were separated electrophoretically in SDS-PAGE running buffer (25 mM Tris base, 190 $\mathrm{mM}$ glycine, $0.1 \% \mathrm{w} / \mathrm{v}$ SDS) at $180 \mathrm{~V}$ for 50-60 minutes using either a Bio-Rad Mini Protean III system or a Mini Protean Tetra Cell system. In order to estimate the size of the 
proteins, an aliquot of PiNK plus pre-stained molecular mass ladder (FroggaBio, Toronto, ON; PM005-0500) was loaded in one well. After electrophoresis, proteins were electroblotted onto a 0.45 micron pore size polyvinylidene difluoride (PVDF) membrane (Immobilon-P membrane, IPVH00010; Millipore, Etobicoke, ON) by wet transfer with a transfer buffer solution ( $25 \mathrm{mM}$ Tris base $\mathrm{pH} 8.5,192 \mathrm{mM}$ glycine, and 20\% v/v methanol) at $4^{\circ} \mathrm{C}$ for 100 minutes at $160 \mathrm{~mA}$. If a protein was less than $20 \mathrm{kDa}$, the wet transfer parameters would instead be switched to $30 \mathrm{~V}$ for 45 minutes at $4^{\circ} \mathrm{C}$. The membrane was washed three times for 5 minutes each with 3-5 $\mathrm{mL}$ of Tris-buffered saline containing Tween-20 (TBST: $20 \mathrm{mM}$ Tris base, $140 \mathrm{mM} \mathrm{NaCl}, 0.05 \% \mathrm{v} / \mathrm{v}$ Tween-20). The blots were then blocked with 5\% - 10\% w/v skim milk dissolved in TBST for 30 minutes or high molecular weight polyvinyl alcohol (PVA) $(70-100 \mathrm{kDa}$ : made at $1.0 \mathrm{mg} / \mathrm{mL}$ concentration in TBST, and boiled for 10 minutes) for up to 90 seconds, followed by three 5 minute washes using TBST. Blots were then incubated overnight at $4^{\circ} \mathrm{C}$ with primary antibody diluted in TBST (1:1000 v:v) with a small amount of sodium azide added. The blocking and antibody dilutions differed for each antibody (details of conditions for each antibody are provided in APPENDIX B). Membranes were then washed multiple times with TBST and deionized water, and incubated with appropriate secondary antibody (typically goat anti-rabbit $\mathrm{IgG}$ ) conjugated with horseradish peroxidase (HRP; Bioshop Canada; Burlington, ON) for 30 minutes; antibody was diluted 1:5000 v:v in TBST (or 1:8000 v:v in TBST for 45 minutes). Membranes were again washed with TBST and signal was detected using enhanced chemiluminescence (ECL); $1.2 \mathrm{~mL}$ of 1:1 v:v substrate solutions (hydrogen peroxide and luminol reagent) were first thoroughly mixed and then added to the membrane to initiate the reaction. The resulting chemiluminescence signal was detected 
using the ChemiGenius or a G:BOX Bioimaging system (Syngene Co., MD, USA) and band densities were analyzed using the associated Gene Tools software. Membranes were then stained for 10 minutes with Coomassie blue $(0.25 \% \mathrm{w} / \mathrm{v}$ Coomassie Brilliant Blue R in $50 \% \mathrm{v} / \mathrm{v}$ methanol, $7.5 \% \mathrm{v} / \mathrm{v}$ acetic acid) and then destained for $\sim 15 \mathrm{~min}$ with destaining mix $\left(60 \% \mathrm{v} / \mathrm{v}\right.$ methanol, $20 \% \mathrm{v} / \mathrm{v}$ acetic acid in $\left.\mathrm{ddH}_{2} \mathrm{O}\right)$.

\section{$\underline{2.2 .4-\text { Statistical Analysis }}$}

Detection of bands on gels and blots used the ChemiGenius Bio-Imaging System (Syngene) and densitometric analysis was performed with the associated GeneTools software. Immunoblot band intensity in each lane was normalized against a set of strong Coomassie blue-stained bands in the same lane to correct any minor variations in sample loading; the Coomassie stained band chosen showed constant intensity across all samples and was well-separated from the area of the gel containing the immunoreactive protein. Mean normalized band densities \pm standard error of the mean (S.E.M.) were calculated for control, 5hA and 20hA samples and significance testing used one-way analysis of variance (ANOVA) followed by Tukey's post-hoc testing with a significance level threshold of $p=0.05$. Tukey's range test statistic is calculated using the following equation:

$$
q_{S}=\frac{Y_{A}-Y_{B}}{S E}=\frac{\left(\bar{y}_{\max }-\bar{y}_{\min }\right)}{S \sqrt{\frac{2}{n}}}
$$

where $\mathrm{q}_{\mathrm{s}}$ is the studentised range statistic, $\mathrm{SE}$ is the standard error of the data, $\mathrm{S}^{2}$ is the pooled variance of the samples, $\mathrm{y}_{\max }\left(\mathrm{Y}_{\mathrm{A}}\right)$ is the larger mean being tested, and $\mathrm{y}_{\min }\left(\mathrm{Y}_{\mathrm{B}}\right)$ is the smaller mean being tested. 


\section{3 - Results}

The antibodies used in the following study cross-reacted with only a single protein band at the expected molecular masses after electrophoresis of $T$. scripta elegans tissue extracts. The proteins analyzed were as follows with their bioinformatically identified molecular weight in brackets using the National Center for Biotechnology Information (NCBI: http://www.ncbi.nlm.nih.gov): SMAD1 (52 kDa), SMAD2 (52 kDa), SMAD3 (48 kDa), SMAD4 (60 kDa), SMAD5 (52 kDa), c-Myc (47kDa), and TGF- $\beta$ precursor (45 kDa). Specific T. scripta elegans protein sequences can be found in APPENDIX A.

\subsection{1 - SMAD Liver Expression in Anoxic Conditions}

When TGF- $\beta$ proteins bind to TGF- $\beta$ receptors, R-SMADs are phosphorylated and form oligomeric complexes with other R-SMADs and SMAD4. In liver, expression levels of total SMAD1 protein significantly dropped in tissue from both 5 hour and 20 hour anoxia conditions compared to controls $(0.610 \pm 0.056, p<0.005 ; 0.693 \pm 0.060, p<0.05)$ (Figure 2.1). Oppositely, SMAD5, the other SMAD protein implicated in the BMP signaling portion of the pathway, increased under 5 hour anoxia and further increased significantly under 20 hour anoxia compared to controls $(1.294 \pm 0.210$, N.S.; $1.921 \pm 0.128, p<0.005)$. The latter level (20 hour anoxia) also differed from the 5 hour anoxic protein levels $(p<0.05)$. The phosphorylated forms of SMADs 1 and 5 (serine 463/465), the activated forms of each, appeared as single bands on the immunoblot at $52 \mathrm{kDa}$ where the $\mathrm{p}$ SMAD1/5 antibody cross reacted (Figure 2.1, 2.5) The observed levels of p-SMAD1/5 in turtle liver were $(64.1 \pm 4.7) \%$, and $(25.8 \pm 2.6) \%$ of controls in 5 hour anoxic and 20 hour anoxic turtles, respectively (both comparisons to control liver levels: $p<0.001$ ), while a statistical difference was also present between the two anoxic conditions $(p<0.001)$. 
Looking at the portion of the pathway that is responsive to endogenous TGF-beta protein, SMAD2 and its phosphorylated form (serine 465/467) showed significant changes related to anoxia (Figure 2.2). SMAD2 levels were unchanged after 5 hour of anoxia but then showed a dramatic decrease at 20 hours of anoxia (5hA: $0.947 \pm 0.080$, N.S.; 20hA: $0.259 \pm 0.146, p<0.005)$. Relative levels of p-SMAD2 behave similarly but with a more moderate, but still significant, drop in expression level after 20 hour anoxia (5hA: $0.879 \pm$ 0.058$, N.S.; $20 \mathrm{hA}: 0.694 \pm 0.069, p<0.05)$. SMAD2 levels differed between the two anoxia treatments $(p<0.01)$, whereas $p$-SMAD2 levels, by contrast, did not. SMAD3 showed more pronounced differences than SMAD2 between normoxic and anoxic conditions (5hA: $0.606 \pm 0.076, p<0.005 ; 20 \mathrm{hA}: 0.346 \pm 0.078, p<0.001)$; in addition the anoxic levels were significantly different from each other $(p<0.001)$ (Figure 2.3). Phospho-SMAD3 phosphorylation decreased to $61 \%$, and subsequently to $35 \%(0.606 \pm 0.075, p<0.05$; 20hA: $0.346 \pm 0.077, p<0.001)$. The phosphorylation state of SMAD3 did not change significantly between the anoxic conditions. The common SMAD that forms the final portion of the oligomeric complex that enters the nucleus, SMAD4, also showed a drop in expression level under anoxic conditions. However, the drop was more severe than for any of the R-SMADs. SMAD4 decreased to $41.2 \pm 0.6 \%$ in 5 hour anoxic turtles $(0.412 \pm$ $0.006, p<0.001)$ and to $15.9 \pm 1.5 \%$ in 20 hour anoxic turtles compared to normoxic turtles $(0.159 \pm 0.015, p<0.001)$; values for 5 hour and 20 hour anoxia also differed statistically $(p<0.01)$ (Figure 2.4) 
A)

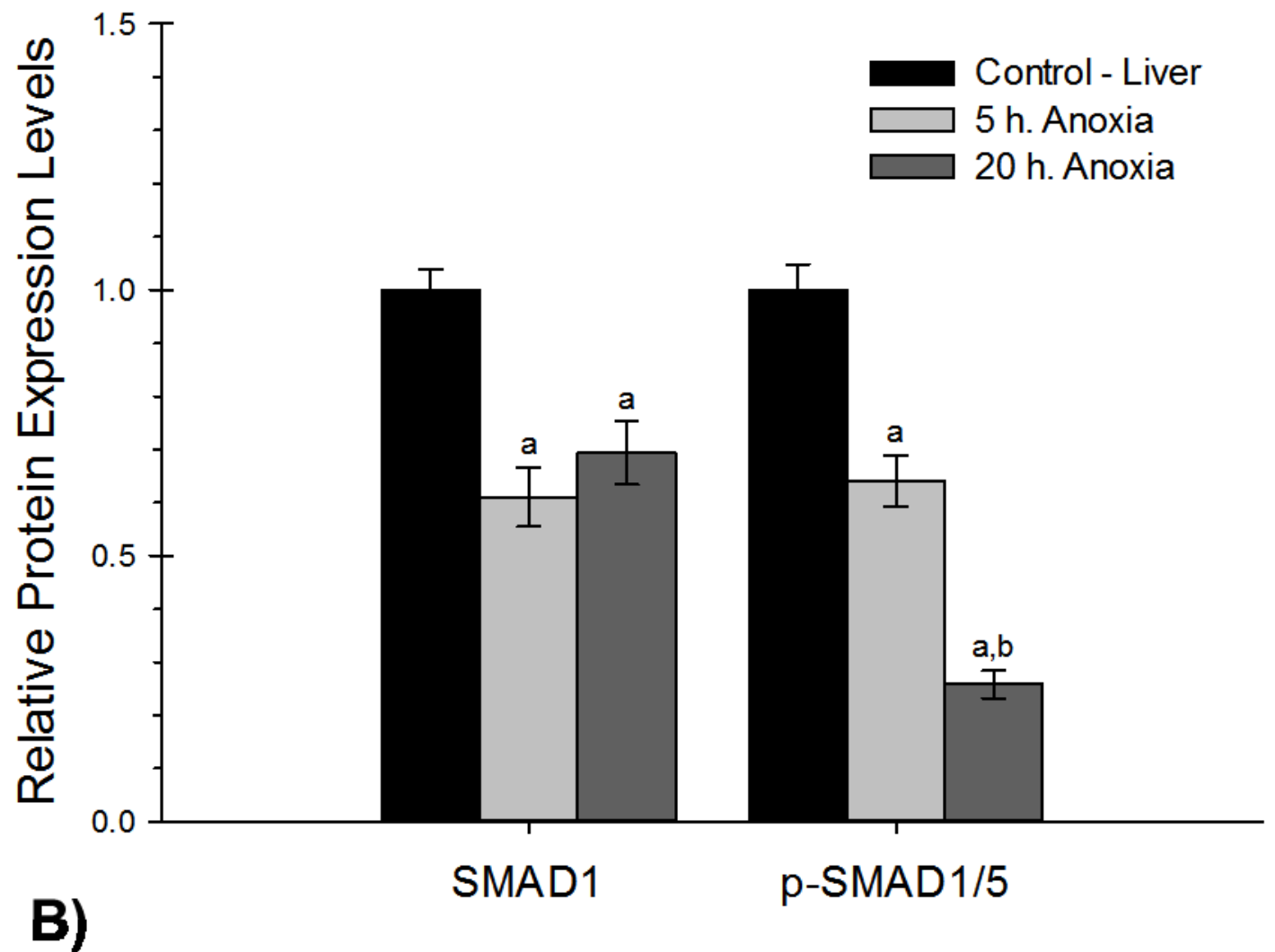

SMAD1
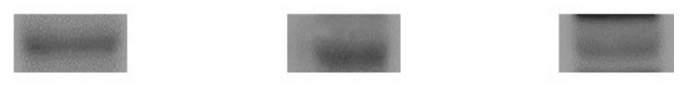
p-SMAD1/5
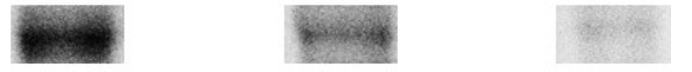

Figure 2.1 - Effects of five hour and twenty hour anoxic exposure on the protein expression of SMAD1 (52 kDa) and phospho-SMAD1/5 (serine 463/465) in redeared slider liver as determined by immunoblotting. A histogram of normalized mean protein levels (A) is shown along with representative immunoblots (B). Data are means \pm S.E.M., $n=3-4$ independent trials. Significantly different from the corresponding $(a)$ control or $(b) 5$ hour anoxia values using a one-way ANOVA and a Tukey's post-hoc test, $p<0.05$. 


\section{A)}

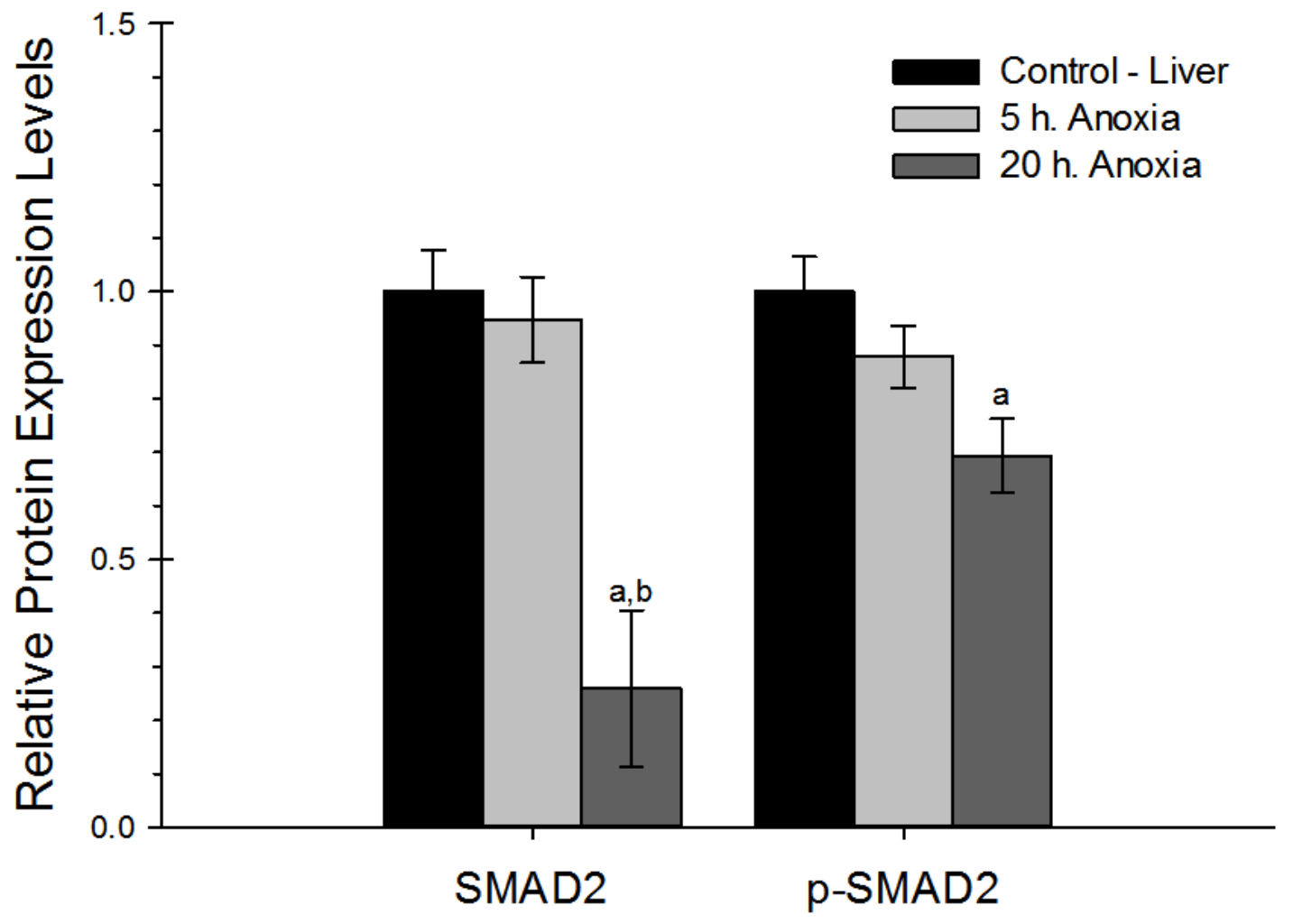

B)

\section{SMAD2}

\section{p-SMAD2}
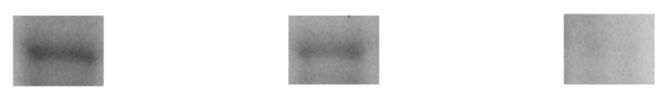

Figure 2.2 - Effects of five hour and twenty hour anoxic exposure on the protein expression of SMAD2 (52 kDa) and phospho-SMAD2 (serine 465/467) in red-eared slider liver as determined by immunoblotting. A histogram of normalized mean protein levels (A) is shown along with representative immunoblots (B). Data are means \pm S.E.M., $n=3-4$ independent trials. Significantly different from the corresponding $(a)$ control or $(b) 5$ hour anoxia values using a one-way ANOVA and a Tukey's post-hoc test, $p<0.05$. 


\section{A)}

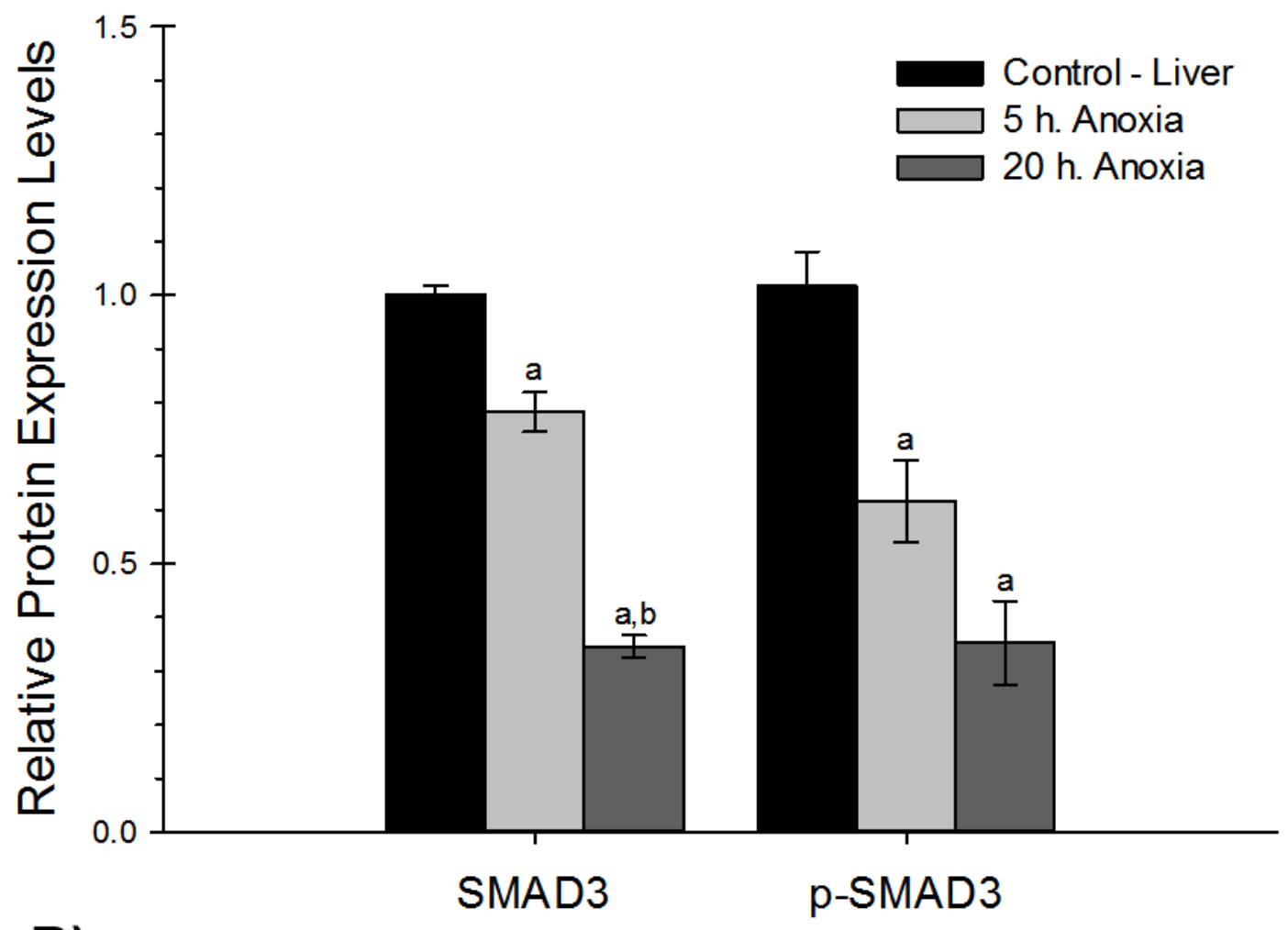

B)

\section{SMAD3}

\section{p-SMAD3}

Figure 2.3 - Effects of five hour and twenty hour anoxic exposure on the protein expression of SMAD3 (48 kDa) and phospho-SMAD3 (serine 423/425) in red-eared slider liver as determined by immunoblotting. A histogram of normalized mean protein levels (A) is shown along with representative immunoblots (B). Data are means \pm S.E.M., $n=3-4$ independent trials. Significantly different from the corresponding $(a)$ control or $(b) 5$ hour anoxia values using a one-way ANOVA and a Tukey's post-hoc test, $p<0.05$. 


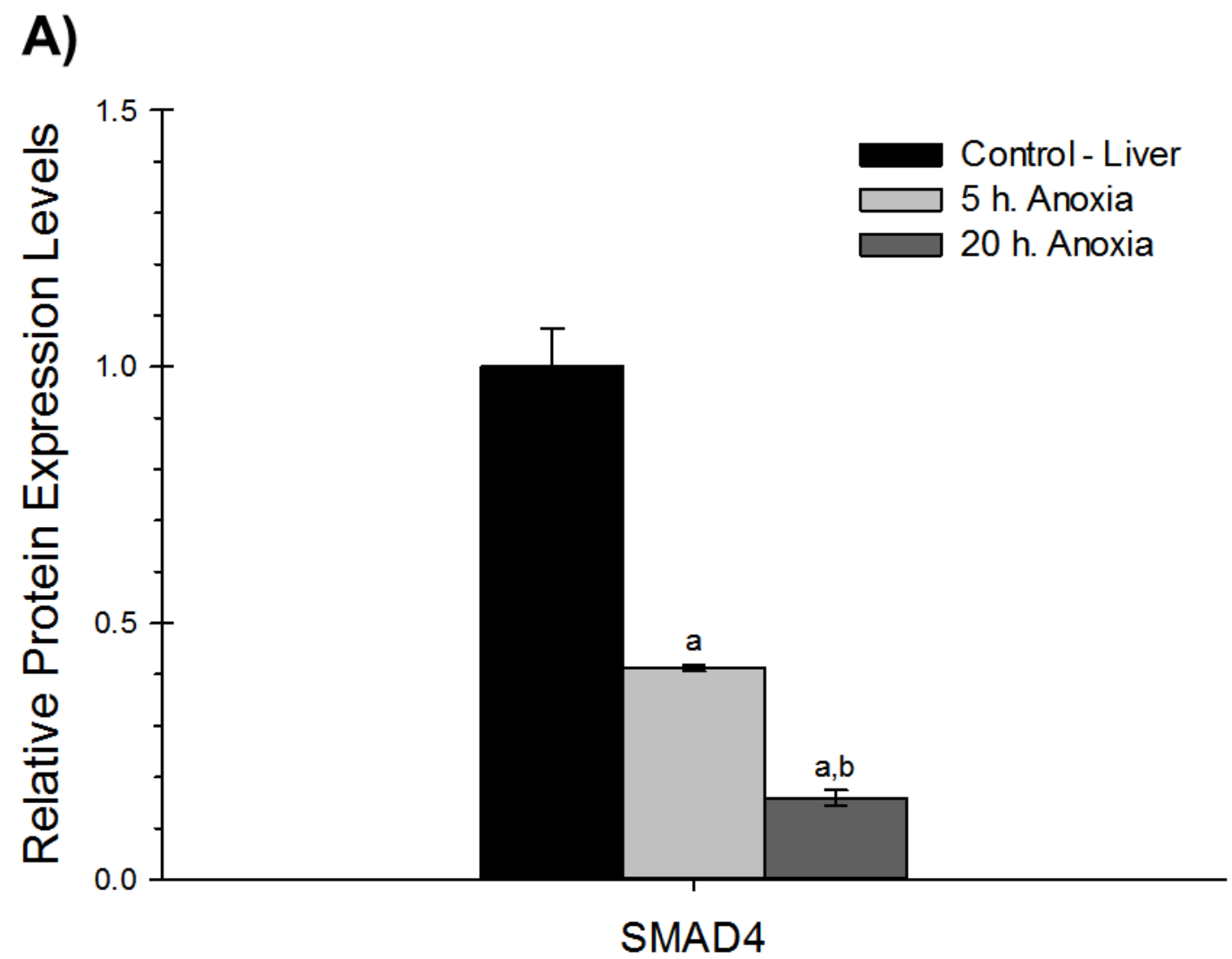

B)

SMAD4
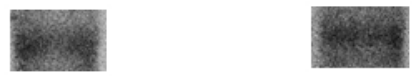

Figure 2.4 - Effects of five hour and twenty hour anoxic exposure on the protein expression of SMAD4 $(60 \mathrm{kDa})$ in red-eared slider liver as determined by immunoblotting. A histogram of normalized mean protein levels (A) is shown along with representative immunoblots (B). Data are means \pm S.E.M., $n=3-4$ independent trials. Significantly different from the corresponding $(a)$ control or $(b) 5$ hour anoxia values using a one-way ANOVA and a Tukey's post-hoc test, $p<0.05$. 


\section{A)}

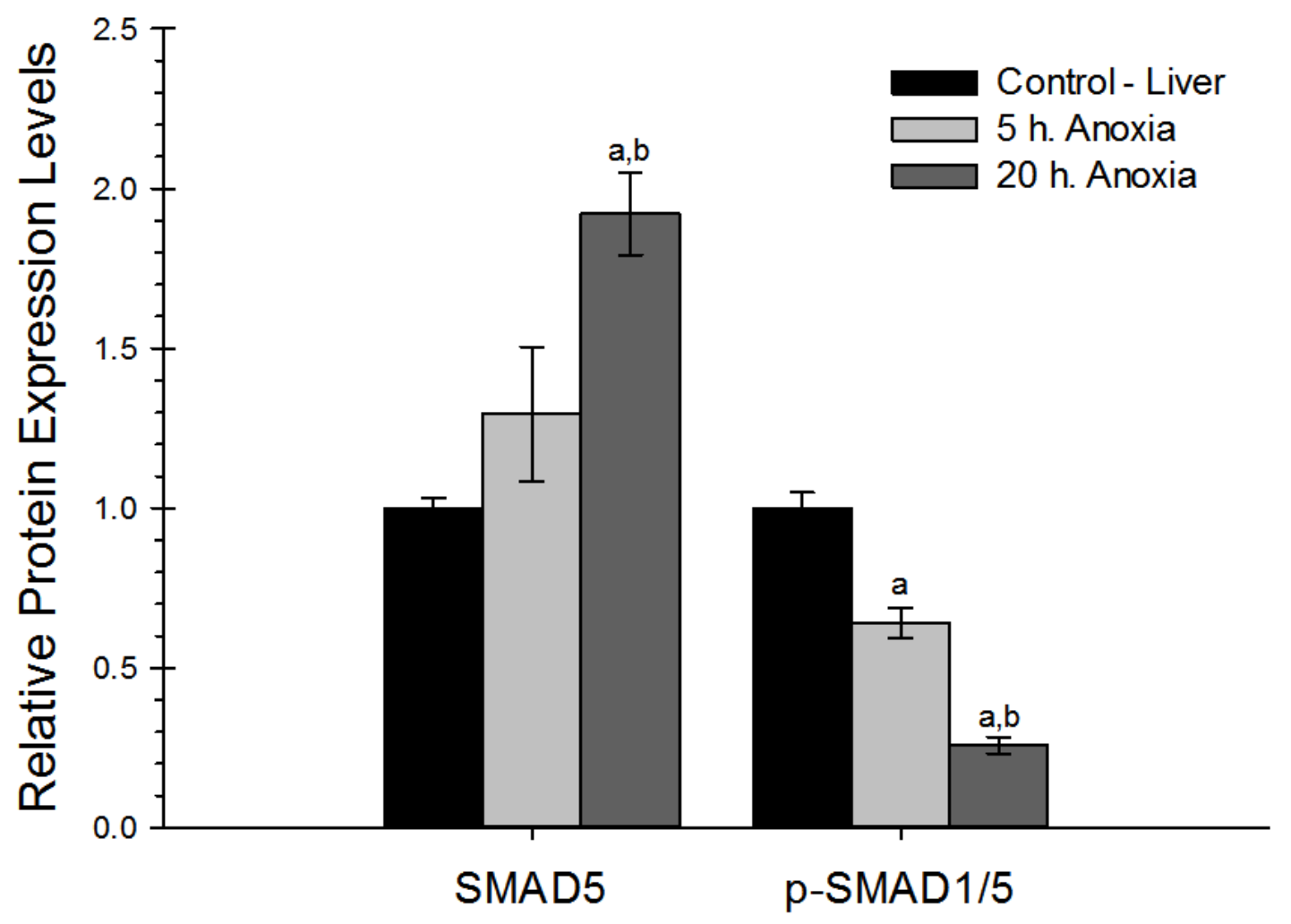

B)

\section{SMAD5}

\section{p-SMAD1/5}

Figure 2.5 - Effects of five hour and twenty hour anoxic exposure on the protein expression of SMAD5 (52 kDa) and phospho-SMAD1/5 (serine 463/465) in redeared slider liver as determined by immunoblotting. A histogram of normalized mean protein levels $(\mathbf{A})$ is shown along with representative immunoblots (B). Data are means \pm S.E.M., $n=3-4$ independent trials. Significantly different from the corresponding $(a)$ control or $(b) 5$ hour anoxia values using a one-way ANOVA and a Tukey's post-hoc test, $p<0.05$. 


\subsection{2 - SMAD White Muscle Expression in Anoxic Conditions}

White muscle is used to perform fast and strenuous tasks for only short periods of time. It has low numbers of mitochondria and rapid contraction by the muscle is supplied by instantaneous creatine phosphate hydrolysis and immediate activation of glycolysis, both forms of anaerobic metabolism. Overall, this generates very little energy relative to oxidative phosphorylation but the advantage is its ability to supply high levels of ATP for quick, brief actions (e.g. sprint running in humans). The turtle white muscle studied here is the neck retractor muscle that is used to instantly pull the head back into the shell when the animal is threatened or for fast head movements to capture prey. To maintain cellular homeostasis under anoxic stress conditions, the tissue could allow autophagy as a survival strategy and SMADs play a role in promoting autophagy. Hence, SMAD signaling might be expected to increase with increasing anoxic stress.

As seen in Figure 2.6, SMAD1 total protein expression levels did not significantly change after 5 hours of anoxic submergence, and showed a moderate drop after 20 hours of anoxia (5hA: $0.796 \pm 0.092, N . S$.; 20hA: $0.644 \pm 0.084, p<0.05)$. SMAD5 followed a similar pattern with no significant change at 5 hours of anoxia, but a strong decrease after 20 hours of anoxic submergence (5hA: $0.878 \pm 0.222$, N.S.; 20hA: $0.405 \pm 0.063, p<0.05)$

(Figure 2.10). However, the phosphorylation of these proteins showed the same relative levels across all three treatments (5hA: $1.071 \pm 0.061$, N.S.; 20hA: $0.979 \pm 0.054$, N.S.).

SMAD2 protein levels relative to its normoxic expression levels were unchanged in expression after 5 hours of anoxic submergence, but then increased by 1.57 -fold increase after 20 hours of anoxic submergence (5hA: $0.896 \pm 0.065$, N.S.; 20hA: $1.568 \pm 0.121$, $p<0.01$ ); values were significantly different from both control levels and levels after five 
hours of anoxia $(p<0.005)$ (Figure 2.7). The activated form, $\mathrm{p}-\mathrm{SMAD} 2$, showed a protein expression trend similar to the total protein pattern but with a significant drop after five hours of anoxic stress preceding a rise in relative expression levels after 20 hours of anoxia, compared to control protein levels $(5 \mathrm{hA}: 0.752 \pm 0.025, p<0.05,20 \mathrm{hA}: 1.218 \pm 0.073$, $p<0.05)$; again five and 20 hour anoxia protein expression levels differed $(p<0.001)$ (Figure 2.7). It was observed that moderate decreases in relative total protein expression levels occurred in the case of SMAD3 during both anoxic submergence treatments compared to the normoxic condition $(5 \mathrm{hA}: 0.723 \pm 0.030, p<0.001,20 \mathrm{hA}: 0.787 \pm 0.028$, $p<0.005)$. However, the phosphorylation expression pattern showed a significant decrease of similar magnitude only during the 5 hour anoxic submergence, whereas the relative phosphoprotein expression levels returned to control levels after 20 hours of anoxia (5hA: $0.737 \pm 0.053, p<0.05,20 \mathrm{hA}: 0.929 \pm 0.012$, N.S.) (Figure 2.8). SMAD4 protein levels were significantly reduced in muscle of turtles that experienced five hours of anoxia as compared to both normoxic turtles and turtles after 20 hours of anoxic submergence (5hA: $0.631 \pm 0.16, p<0.05$ versus control, $p<0.005$ versus $20 \mathrm{hA}$ ), however 20 hour anoxic turtles had no differential expression of SMAD4 compared to normoxic turtles (20hA: $1.184 \pm$ 0.063, N.S.) (Figure 2.9). 


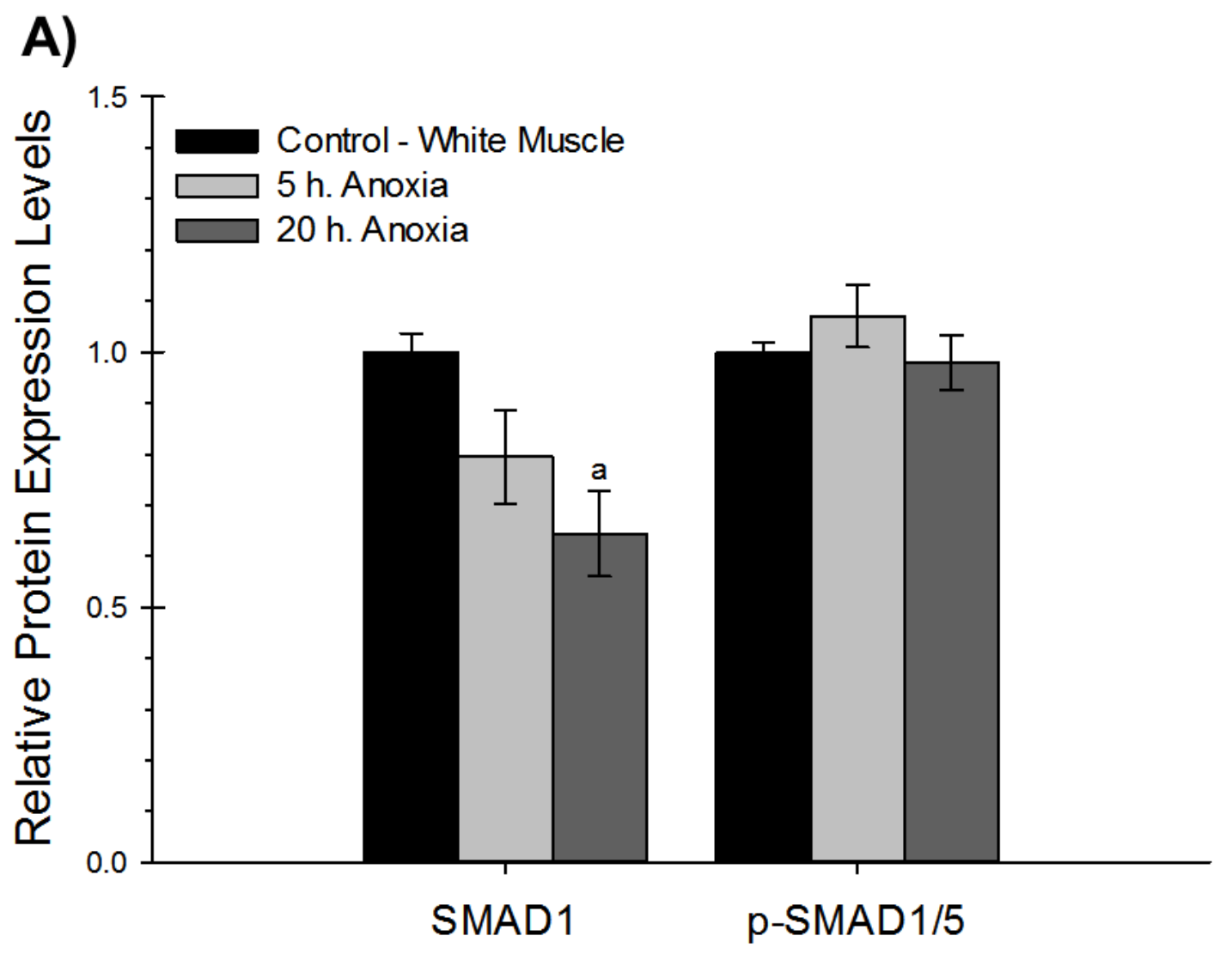

B)
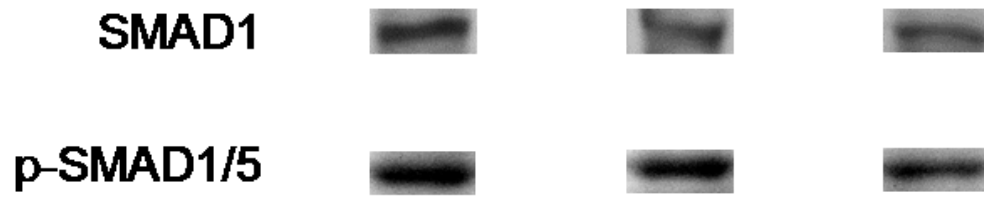

\section{p-SMAD1/5}

Figure 2.6 - Effects of five hour and twenty hour anoxic exposure on the protein expression of SMAD1 (52 kDa) and phospho-SMAD1/5 (serine 463/465) in redeared slider white muscle as determined by immunoblotting. A histogram of normalized mean protein levels (A) is shown along with representative immunoblots (B). Data are means \pm S.E.M., $n=3-4$ independent trials. Significantly different from the corresponding (a) control or (b) 5 hour anoxia values using a one-way ANOVA and a Tukey's post-hoc test, $p<0.05$. 


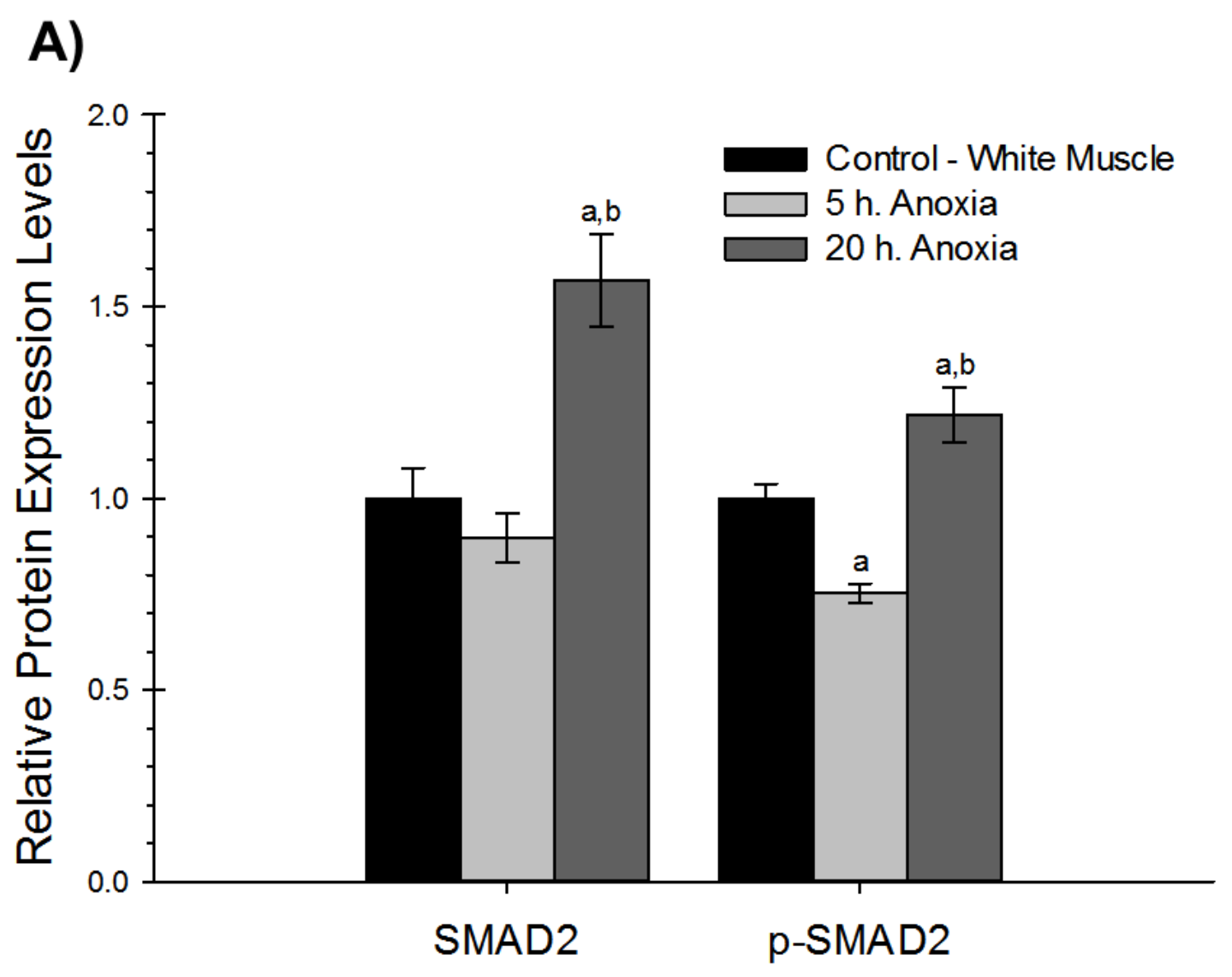

B)
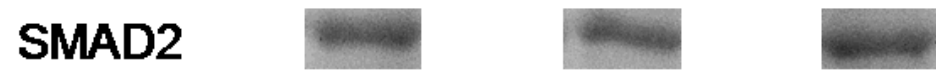

p-SMAD2
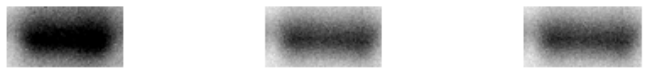

Figure 2.7 - Effects of five hour and twenty hour anoxic exposure on the protein expression of SMAD2 (52 kDa) and phospho-SMAD2 (serine 465/467) in red-eared slider white muscle as determined by immunoblotting. A histogram of normalized mean protein levels (A) is shown along with representative immunoblots (B). Data are means \pm S.E.M., $n=3-4$ independent trials. Significantly different from the corresponding $(a)$ control or $(b) 5$ hour anoxia values using a one-way ANOVA and a Tukey's post-hoc test, $p<0.05$. 


\section{A)}

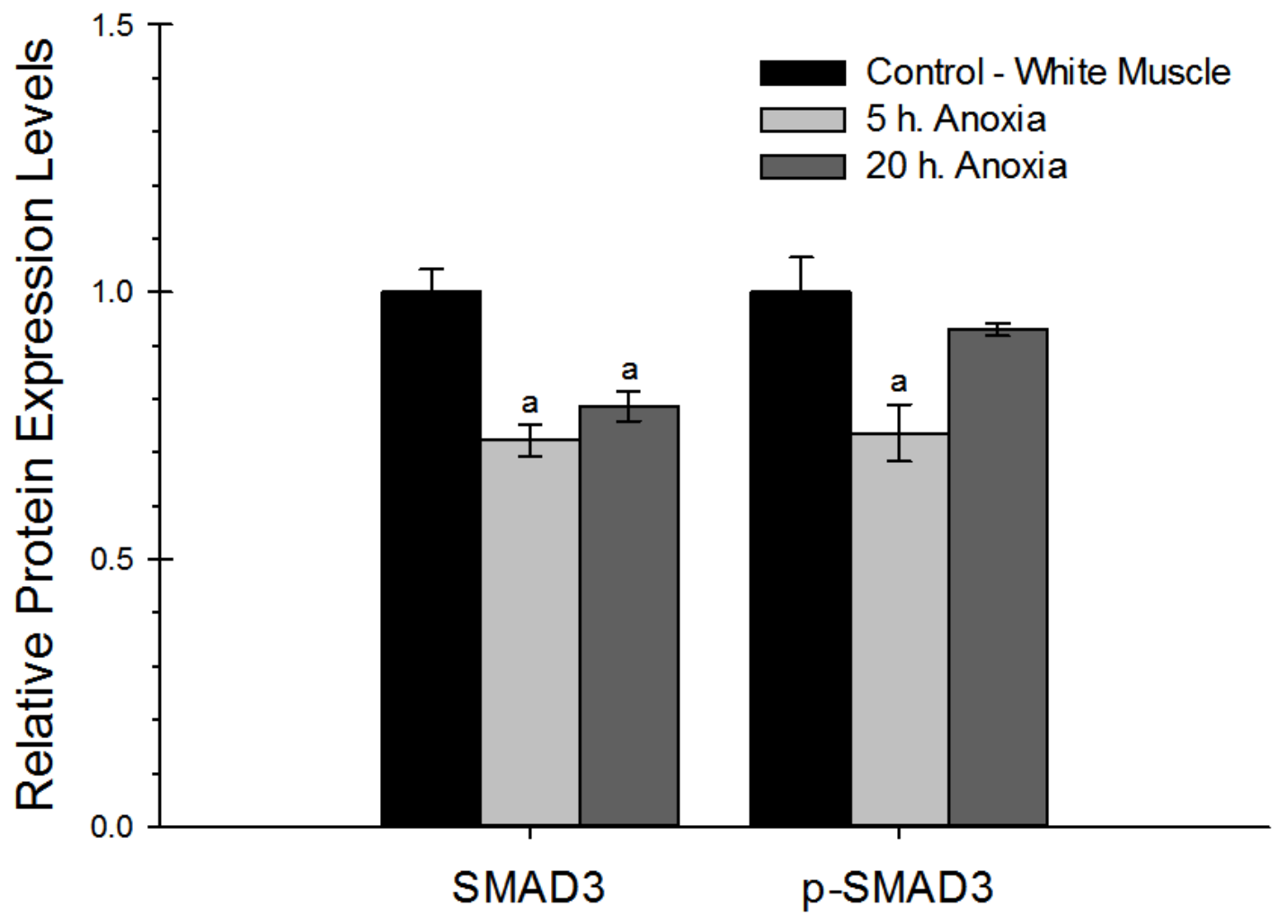

B)

\section{SMAD3}

\section{p-SMAD3}

Figure 2.8 - Effects of five hour and twenty hour anoxic exposure on the protein expression of SMAD3 (48 kDa) and phospho-SMAD3 (serine 423/425) in red-eared slider white muscle as determined by immunoblotting. A histogram showing normalized mean protein levels $(\mathbf{A})$ is shown along with representative immunoblots (B). Data are means \pm S.E.M., $n=3-4$ independent trials. Significantly different from the corresponding $(a)$ control or $(b) 5$ hour anoxia values using a one-way ANOVA and a Tukey's post-hoc test, $p<0.05$. 
A)

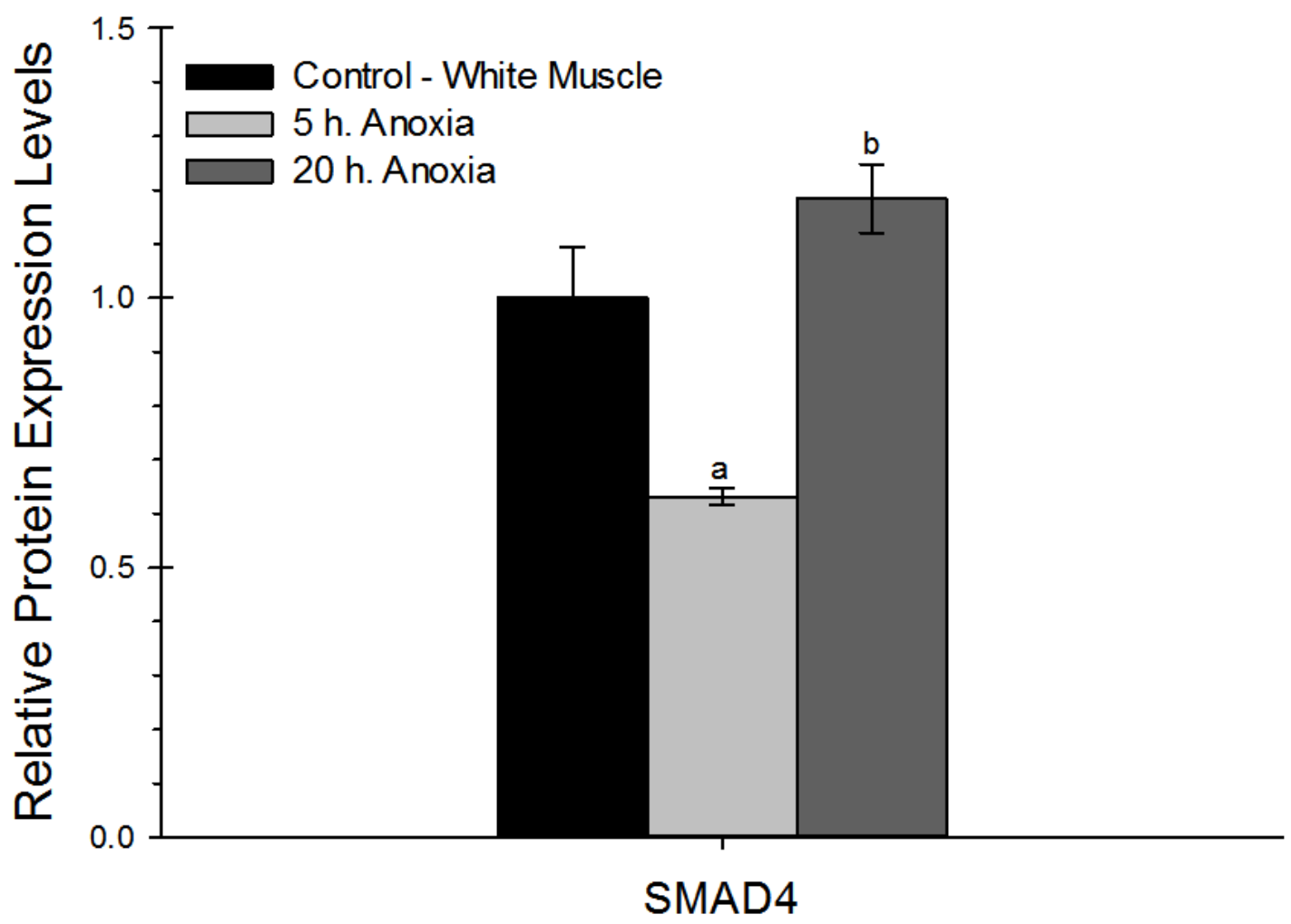

B)

SMAD4

Figure 2.9 - Effects of five hour and twenty hour anoxic exposure on the protein expression of SMAD4 $(60 \mathrm{kDa})$ in red-eared slider white muscle as determined by immunoblotting. A histogram of normalized mean protein levels (A) is shown along with representative immunoblots $(\mathbf{B})$. Data are means \pm S.E.M., $n=3-4$ independent trials. Significantly different from the corresponding $(a)$ control or $(b) 5$ hour anoxia values using a one-way ANOVA and a Tukey's post-hoc test, $p<0.05$. 


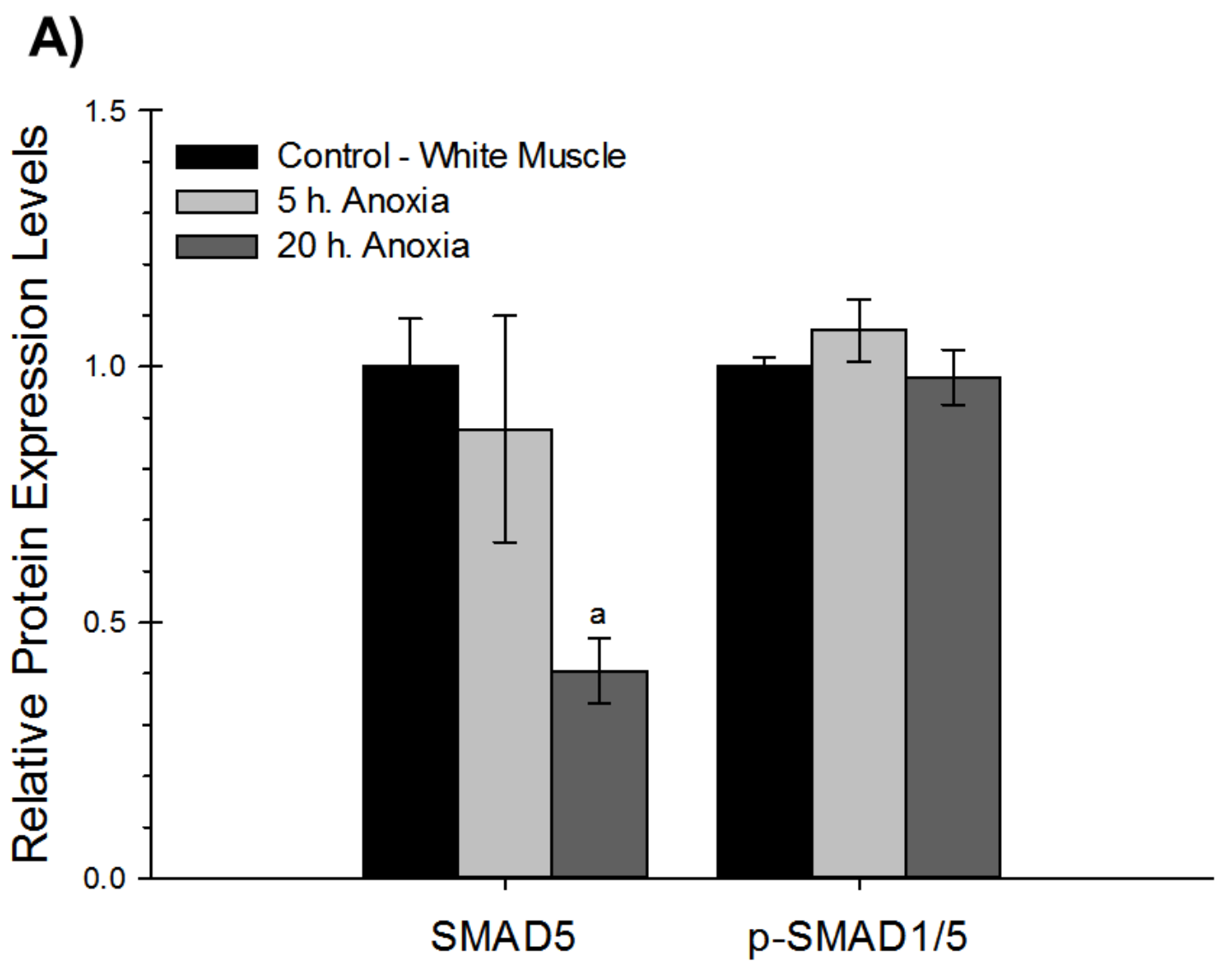

B)

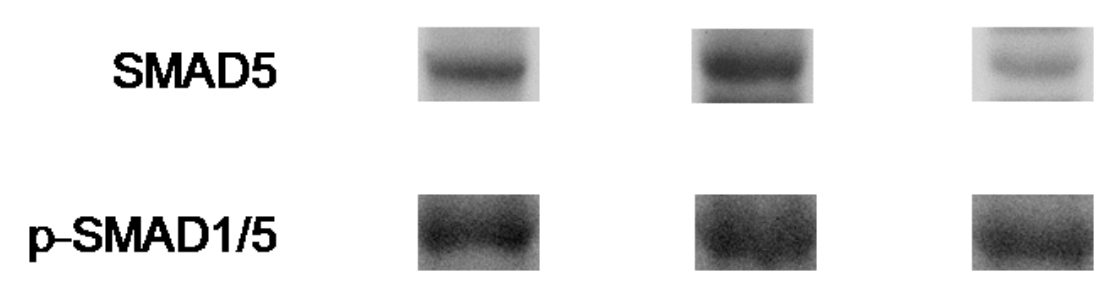

Figure 2.10 - Effects of five hour and twenty hour anoxic exposure on the protein expression of SMAD5 (52 kDa) and phospho-SMAD1/5 (serine 463/465) in redeared slider white muscle as determined by immunoblotting. A histogram of normalized mean protein levels (A) is shown along with representative immunoblots (B). Data are means \pm S.E.M., $n=3-4$ independent trials. Significantly different from the corresponding $(a)$ control or (b) 5 hour anoxia values using a one-way ANOVA and a Tukey's post-hoc test, $p<0.05$. 


\subsection{3 - SMAD Red Muscle Expression in Anoxic Conditions}

Red muscle derives most of its energy needs from oxidative phosphorylation and powers mostly sustained muscle activity; e.g. the turtle red muscle samples analyzed were from the legs and used for swimming and walking. Although red muscle still has a glycolytic capacity, it would be expected that under anoxic conditions that red muscle cells could become less active and show metabolic rate depression to conserve energy. Since the TGF$\beta$ pathway is implicated in inhibiting cell growth, I predicted that SMAD expression levels would increase as the effects of anoxic stress experienced by the muscles increase. Figure 2.12 shows that SMAD2 protein levels increased strongly after 20 hour anoxic exposure, compared to controls $(5 \mathrm{hA}: 1.541 \pm 0.380$, N.S.; 20hA: $3.558 \pm 0.399, p<0.001)$. Furthermore, there was a significant difference between the two anoxic conditions $(p<0.005)$ with SMAD2 levels reaching 3.6-fold higher than controls after 20 hours. Relative p-SMAD2 (serines 465 and 467) levels also increased during short term anoxia with levels 1.9-fold higher than controls after 5 hours anoxia, but plummeted after 20 hours of anoxia (5hA: $1.756 \pm 0.093, p<0.001 ; 20 \mathrm{hA}: 0.335 \pm 0.056, p<0.001)$; p-SMAD2 expression statistically differed between the anoxic groups $(p<0.001)$. SMAD3 showed no significant change in total protein expression over 5 or 20 hours of anoxia (5hA: $1.541 \pm$ 0.310, N.S.; 20hA: $0.706 \pm 0.125$, N.S.) (Figure 2.13). However, phosphorylated SMAD3 (serines 423 and 425) content) decreased to levels just $41.6 \%$ of controls after 5 hours (5hA: $0.416 \pm 0.065, p<0.01)$ and $36.9 \%$ of controls after 20 hour anoxia exposure (20hA: $0.369 \pm 0.056, p<0.001)$. SMAD1 relative total protein levels did not change over 5 hour anoxia exposure but dropped strongly after 20 hour anoxia in the red muscle of turtles (5hA: $1.046 \pm 0.121$, N.S.; $20 \mathrm{hA}: 0.388 \pm 0.105, p<0.01)$; this decrease was also significantly different from the 5 hour anoxic value $(p<0.01)$ (Figure 2.11). SMAD5 
expression levels between normal and anoxic conditions showed that there was a large 3fold increase in protein $(5 \mathrm{hA}: 3.010 \pm 0.618$, N.S. $(p=0.06) ; 20 \mathrm{hA}: 3.091 \pm 0.482$, N.S. $(p=0.07))$ (Figure 2.15). Phosphorylation levels of SMAD1/5 (serine 463/465) showed a decreased trend in anoxia, but neither change was significant (5hA: $0.575 \pm 0.118$, N.S.; 20hA: $0.692 \pm 0.207$, N.S.) (Figure 2.11, 2.15). SMAD4 followed the R-SMADs and showed a 2.5-fold significant increase after 5 hour anoxia exposure but then dropped to half of the control level after 20 hour anoxia (5hA: $2.546 \pm 0.116, p<0.005 ; 20 \mathrm{hA}: 0.478 \pm$ 0.176, N.S.) (Figure 2.14). The 20 hour value was not different from the control but was significantly different from the 5 hour value $(p<0.001)$. 
A)

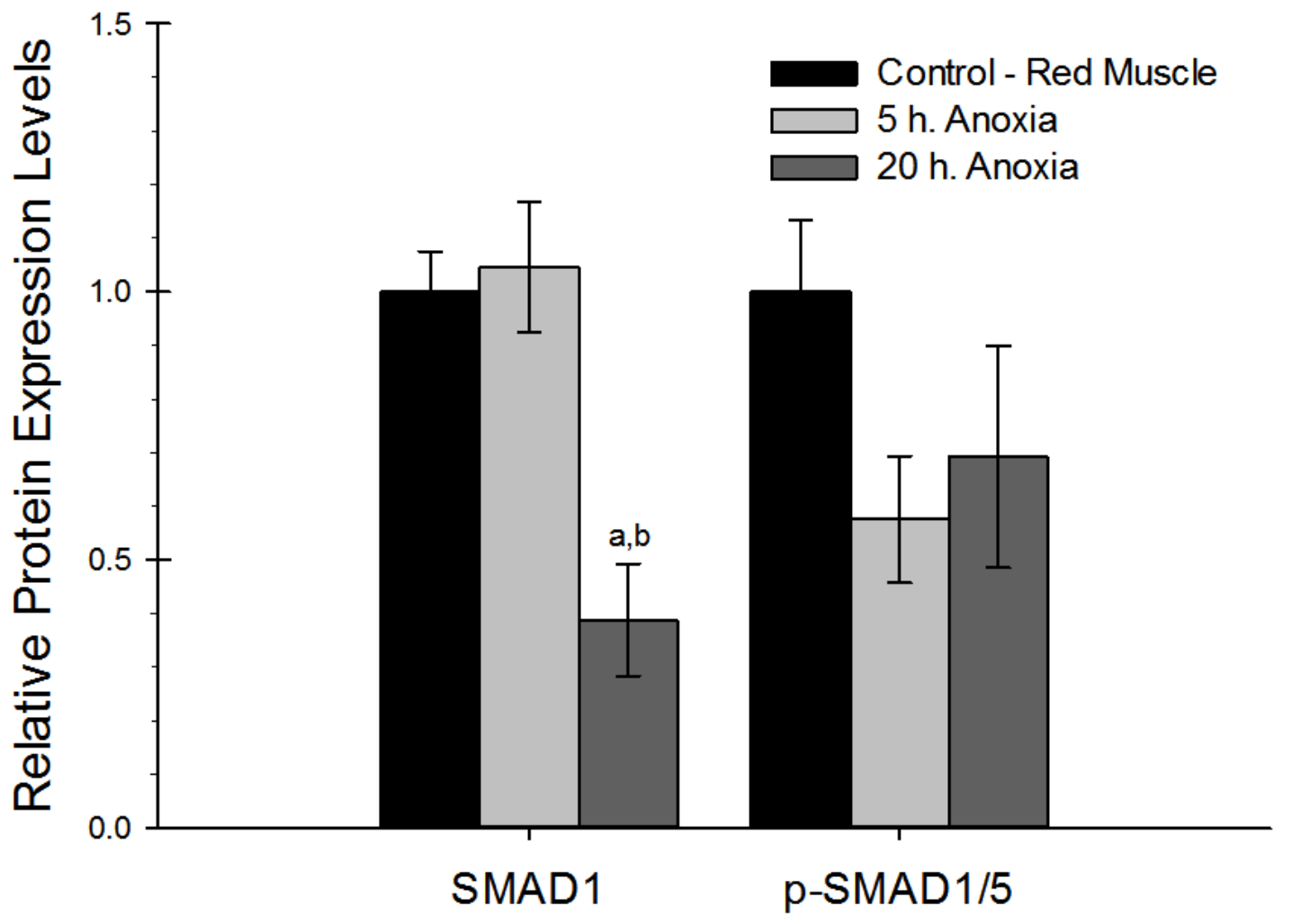

B)

SMAD1
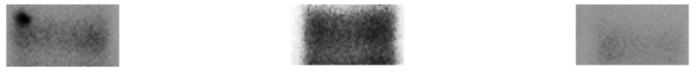
p-SMAD1/5
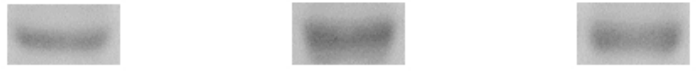

Figure 2.11 - Effects of five hour and twenty hour anoxic exposure on the protein expression of SMAD1 (52 kDa) and phospho-SMAD1/5 (serine 463/465) in redeared slider red skeletal muscle as determined by immunoblotting. A histogram of normalized mean protein levels (A) is shown along with representative immunoblots (B). Data are means \pm S.E.M., $n=3-4$ independent trials. Significantly different from the corresponding $(a)$ control or $(b) 5$ hour anoxia values using a one-way ANOVA and a Tukey's post-hoc test, $p<0.05$. 
A)

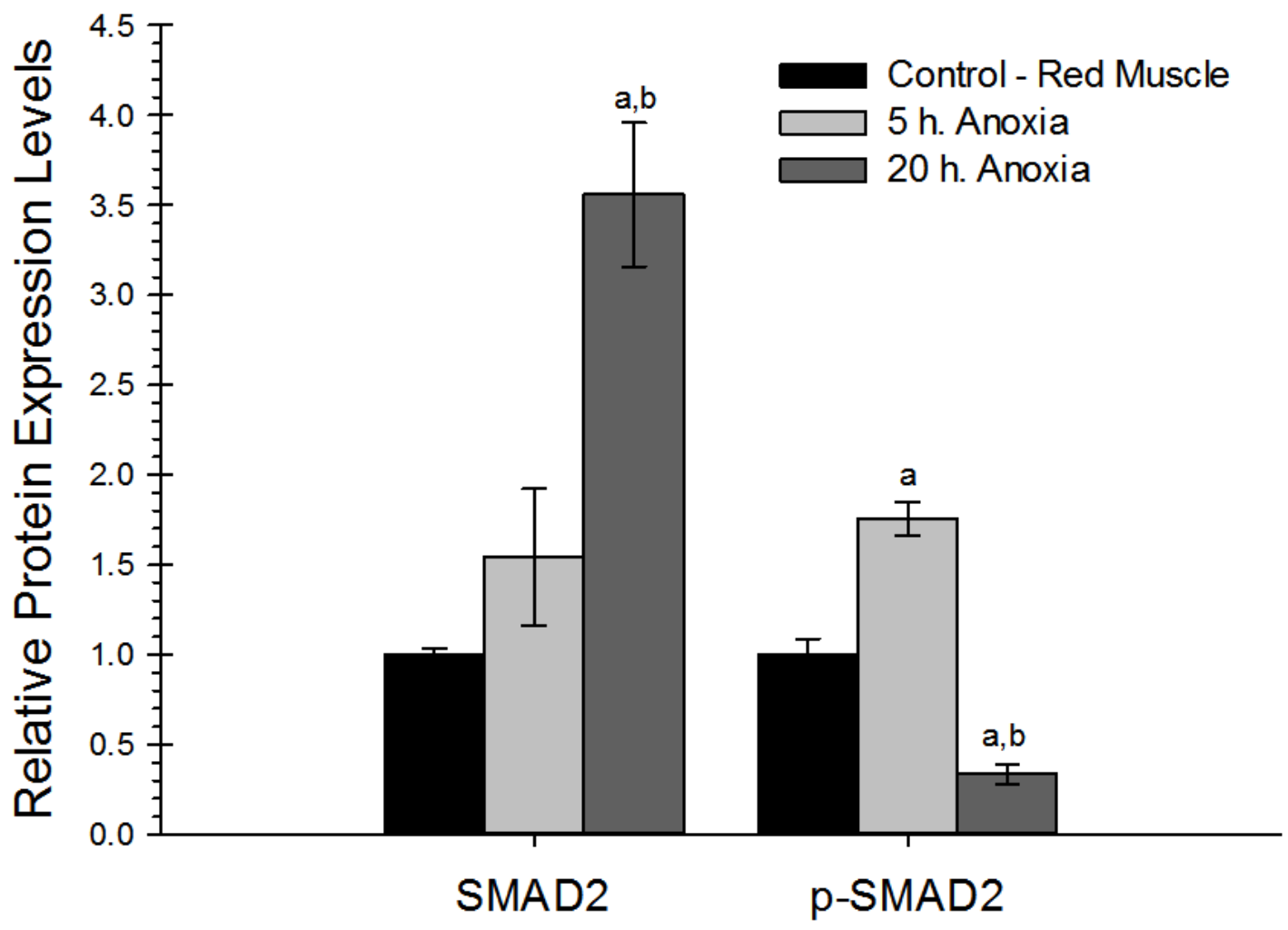

B)

SMAD2

p-SMAD2

Figure 2.12 - Effects of five hour and twenty hour anoxic exposure on the protein expression of SMAD2 (52 kDa) and phospho-SMAD2 (serine 465/467) in red-eared slider red skeletal muscle as determined by immunoblotting. A histogram of normalized mean protein levels $(\mathbf{A})$ is shown along with representative immunoblots (B). Data are means \pm S.E.M., $n=3-4$ independent trials. Significantly different from the corresponding $(a)$ control or $(b) 5$ hour anoxia values using a one-way ANOVA and a Tukey's post-hoc test, $p<0.05$. 
A)

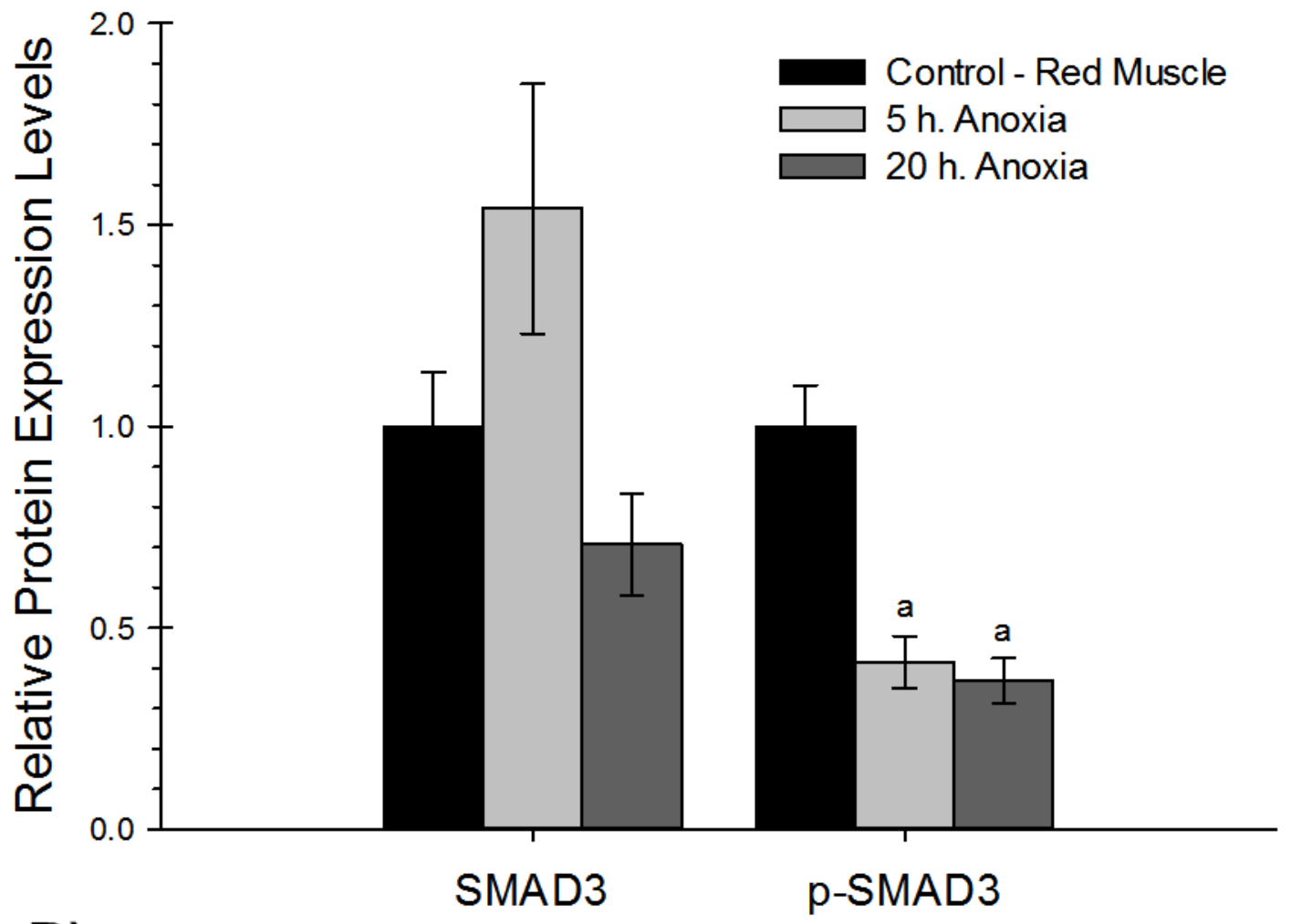

B)

SMAD3
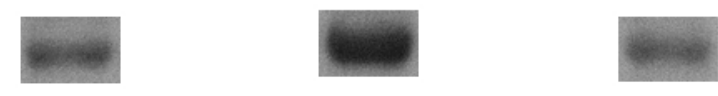

p-SMAD3
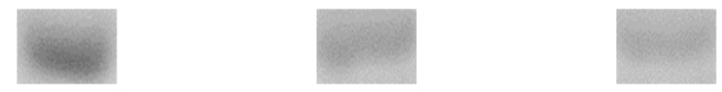

Figure 2.13 - Effects of five hour and twenty hour anoxic exposure on the protein expression of SMAD3 (48 kDa) and phospho-SMAD3 (serine 423/425) in red-eared slider red skeletal muscle as determined by immunoblotting. A histogram of normalized mean protein levels $(\mathbf{A})$ is shown along with representative immunoblots (B). Data are means \pm S.E.M., $n=3-4$ independent trials. Significantly different from the corresponding (a) control or (b) 5 hour anoxia values using a one-way ANOVA and a Tukey's post-hoc test, $p<0.05$. 
A)

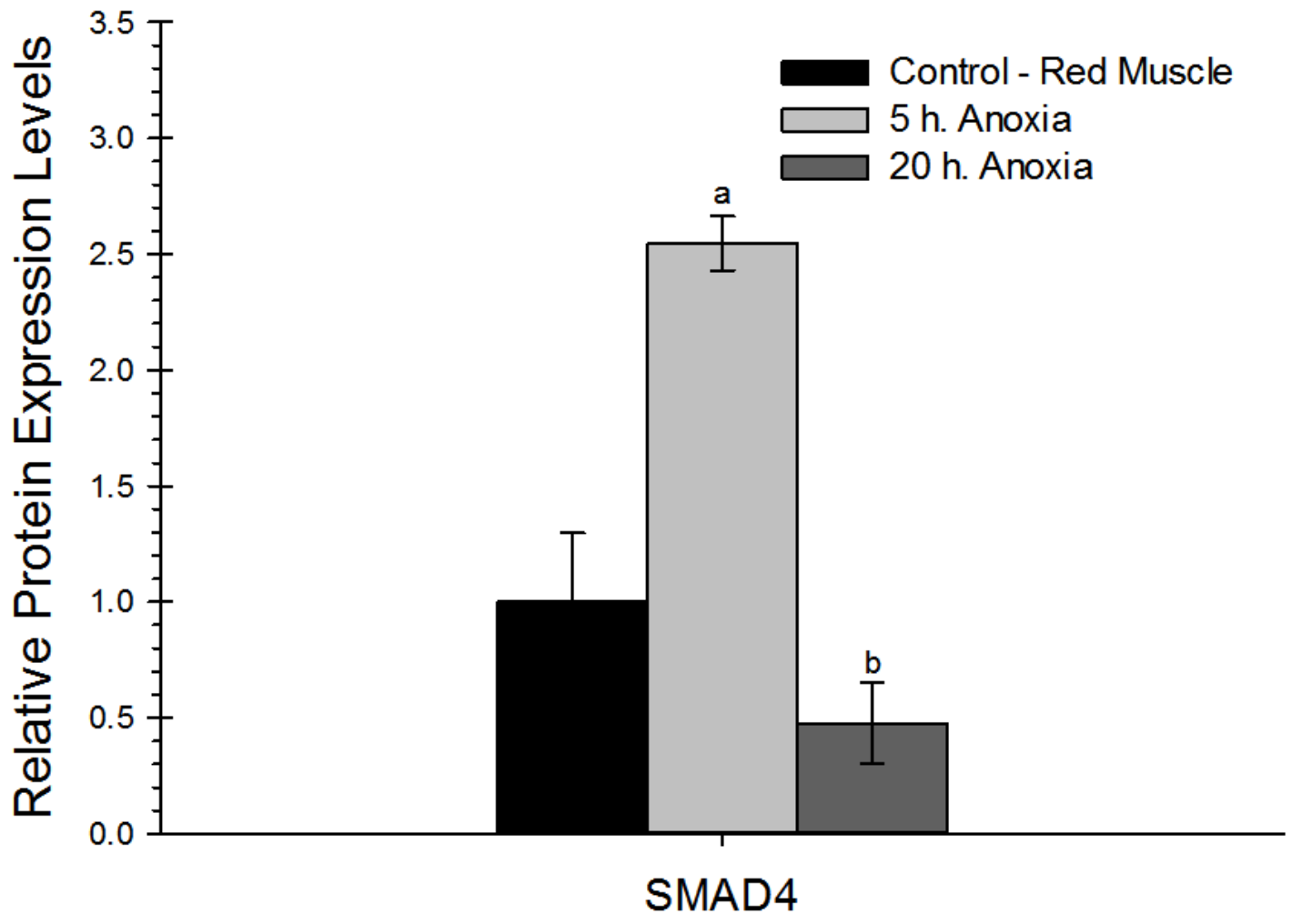

B)

\section{SMAD4}
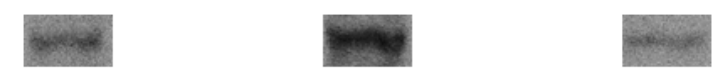

Figure 2.14 - Effects of five hour and twenty hour anoxic exposure on the protein expression of SMAD4 $(60 \mathrm{kDa})$ in red-eared slider red skeletal muscle as determined by immunoblotting. A histogram of normalized mean protein levels (A) is shown along with representative immunoblots $(\mathbf{B})$. Data are means \pm S.E.M., $n=$ 3-4 independent trials. Significantly different from the corresponding (a) control or (b) 5 hour anoxia values using a one-way ANOVA and a Tukey's post-hoc test, $p<$ 0.05 . 


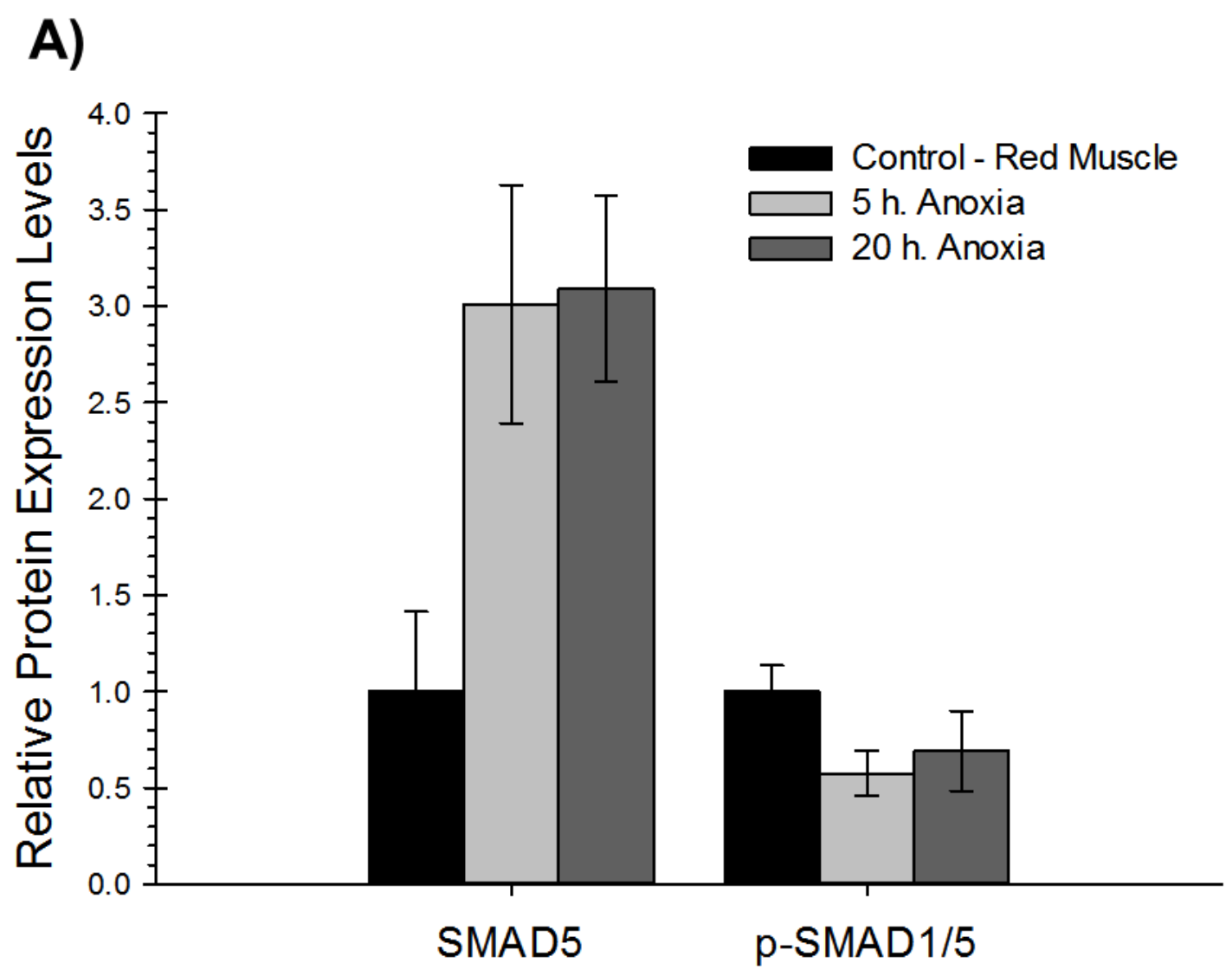

B)

\section{SMAD5 p-SMAD1/5}

Figure 2.15 - Effects of five hour and twenty hour anoxic exposure on the protein expression of SMAD5 (52 kDa) and phospho-SMAD1/5 (serine 463/465) in redeared slider red skeletal muscle as determined by immunoblotting. A histogram of normalized mean protein levels (A) is shown along with representative immunoblots (B). Data are means \pm S.E.M., $n=3-4$ independent trials. Significantly different from the corresponding $(a)$ control or $(b) 5$ hour anoxia values using a one-way ANOVA and a Tukey's post-hoc test, $p<0.05$. 


\subsection{4 - SMAD Kidney Expression in Anoxic Conditions}

SMAD1 expression levels in kidney increased by 2.63 -fold compared to control turtles during 5 hour anoxia exposure, but then returned to control levels after 20 hours (5hA: $2.630 \pm 0.420, p<0.05 ; 20 \mathrm{hA}: 1.021 \pm 0.049$, N.S.) (Figure 2.16). SMAD1 levels also differed significantly between the two anoxic conditions $(p<0.05)$. SMAD5 relative total protein levels stayed constant across all treatments (5hA: $0.784 \pm 0.196$, N.S.; 20hA: 0.970 \pm 0.137 , N.S.) (Figure 2.20). The relative phosphorylation of SMAD1/5 showed a decreasing trend with increasing anoxia time, but values were not significant (5hA: 0.835 \pm 0.107 , N.S.; 20hA: $0.647 \pm 0.055$, N.S.). SMAD2 showed significantly increased expression to 2.5 -fold over control levels during 5 hour anoxia before dropping to a nonsignificant 1.5-fold value after 20 hours compared to normoxic turtle levels (5hA: 2.498 $\pm 0.316, p<0.05 ; 20 \mathrm{hA}: 1.570 \pm 0.212$, N.S.) (Figure 2.17). The activated form of SMAD2 (p-SMAD2) increased 1.76-fold relative to controls during 5 hour anoxia before dropping to half of the control level after 20 hour anoxia (5hA: $1.756 \pm 0.093, p<0.005 ; 20 \mathrm{hA}: 0.528$ $\pm 0.102, p<0.05)$. Expression levels of p-SMAD2 were different between anoxic groups $(p<0.001)$. SMAD3 relative expression levels were approximately halved after both anoxic treatments (5 and 20 hour) compared to controls ( $5 \mathrm{hA}: 0.472 \pm 0.126, p<0.05 ; 20 \mathrm{hA}: 20 \mathrm{hA}$ : $0.565 \pm 0.029, p<0.05$ ) (Figure 2.18). Phosphorylated SMAD3, however, was unchanged after 5 hours anoxic stress, but plummeted to a quarter of the control levels after 20 hours of anoxia (5hA: $0.882 \pm 0.027$, N.S.; $20 \mathrm{hA}: 0.255 \pm 0.031, p<0.001)$; this drop was also significantly different from the 5 hour anoxia kidney expression $(p<0.001)$. Finally, SMAD4 in the kidney decreased to about $44 \%$ of the control level during 5 hours of anoxia and remained unchanged at that same expression level after 20 hours of anoxia (5hA: 0.444 $\pm 0.049, p<0.001 ; 20 \mathrm{hA}: 0.478 \pm 0.055, p<0.005)$ (Figure 2.19). 
A)

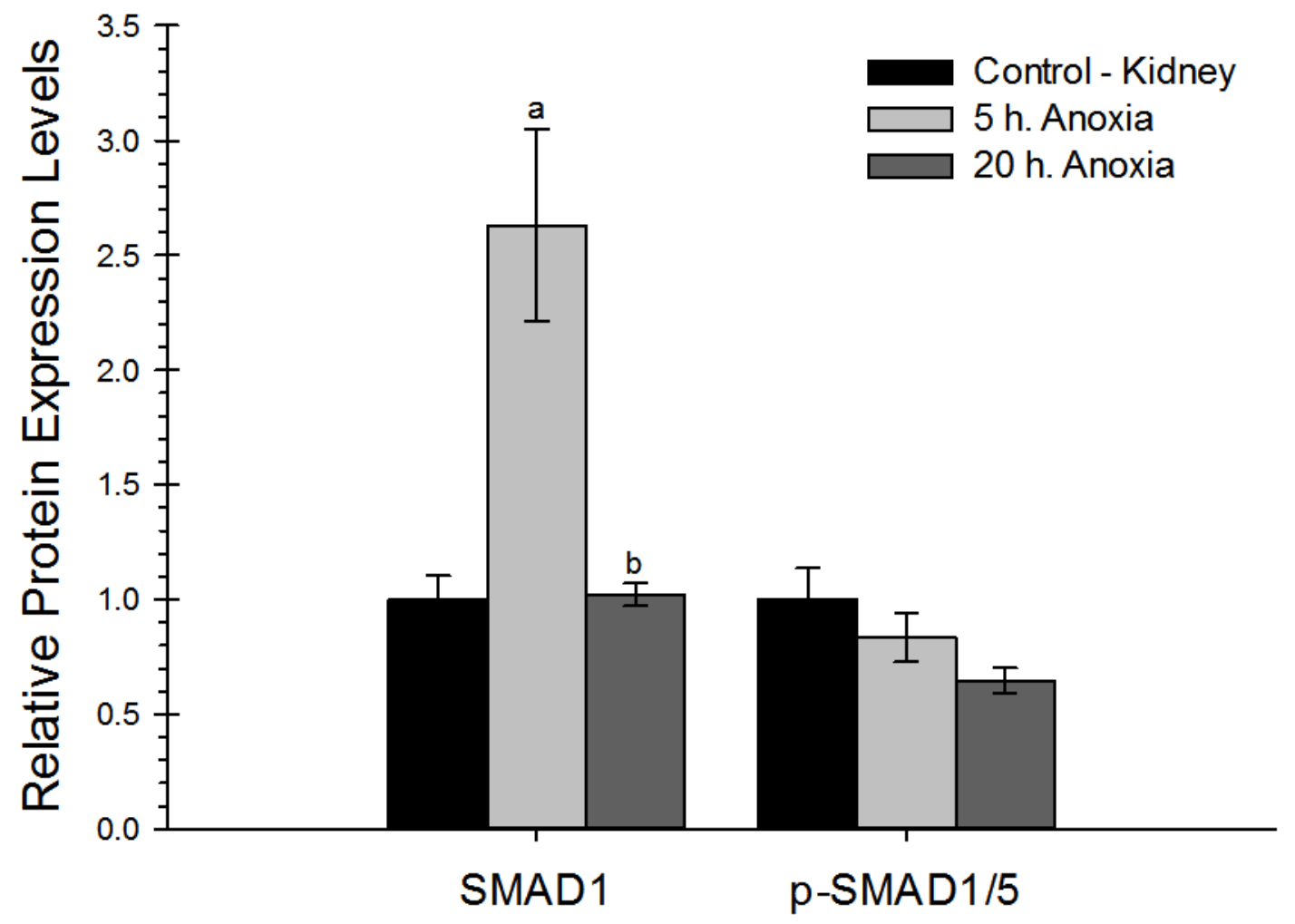

B)

\section{SMAD1}

p-SMAD1/5
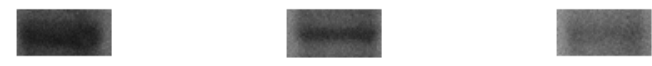

Figure 2.16 - Effects of five hour and twenty hour anoxic exposure on the protein expression of SMAD1 (52 kDa) and phospho-SMAD1/5 (serine 463/465) in redeared slider kidney as determined by immunoblotting. A histogram of normalized mean protein levels (A) is shown along with representative immunoblots (B). Data are means \pm S.E.M., $n=3-4$ independent trials. Significantly different from the corresponding (a) control or (b) 5 hour anoxia values using a one-way ANOVA and a Tukey's post-hoc test, $p<0.05$. 


\section{A)}

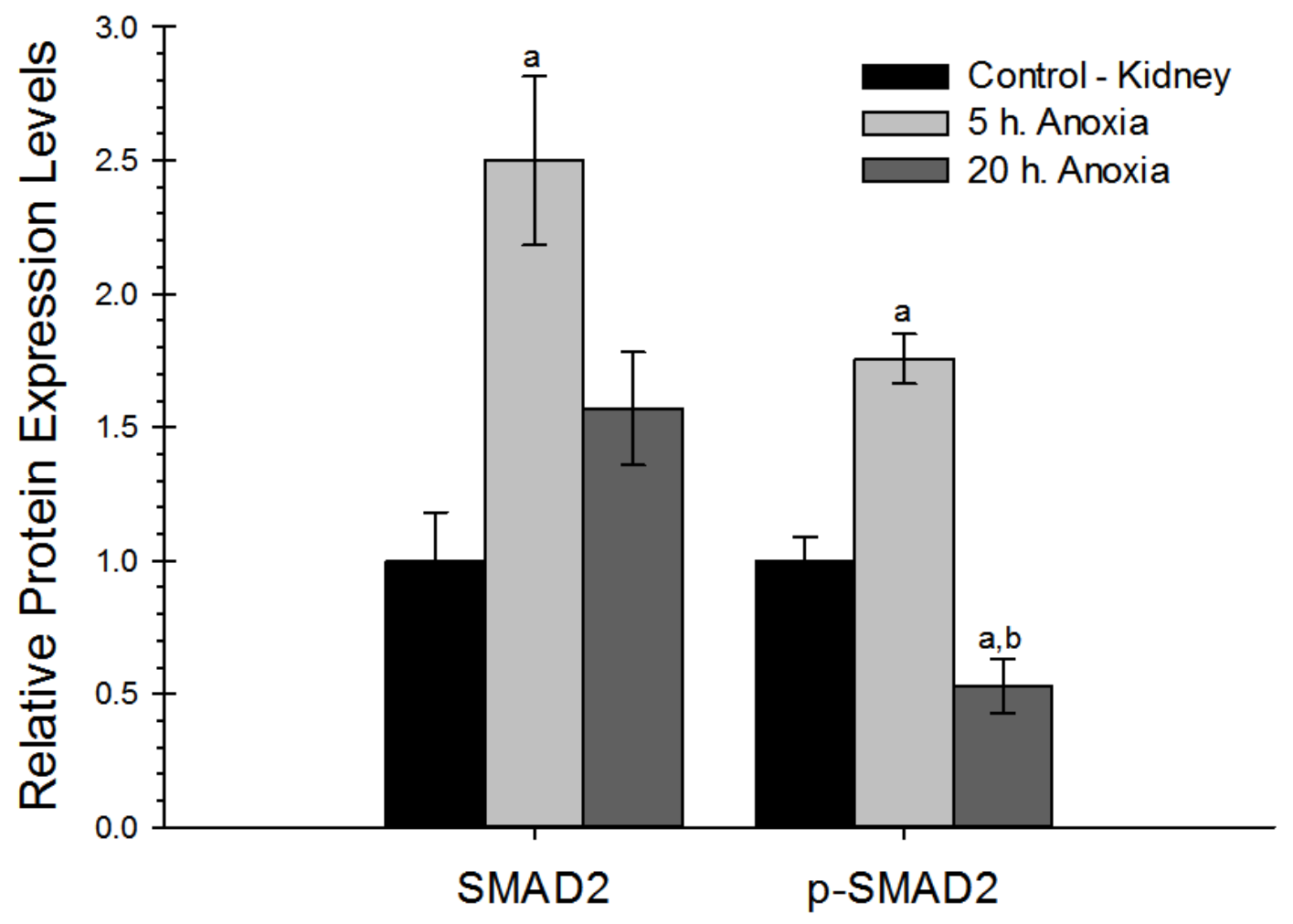

B)

\section{SMAD2}

p-SMAD2

Figure 2.17 - Effects of five hour and twenty hour anoxic exposure on the protein expression of SMAD2 (52 kDa) and phospho-SMAD2 (serine 465/467) in red-eared slider kidney as determined by immunoblotting. A histogram of normalized mean protein levels (A) is shown along with representative immunoblots (B). Data are means \pm S.E.M., $n=3-4$ independent trials. Significantly different from the corresponding $(a)$ control or $(b) 5$ hour anoxia values using a one-way ANOVA and a Tukey's post-hoc test, $p<0.05$. 


\section{A)}

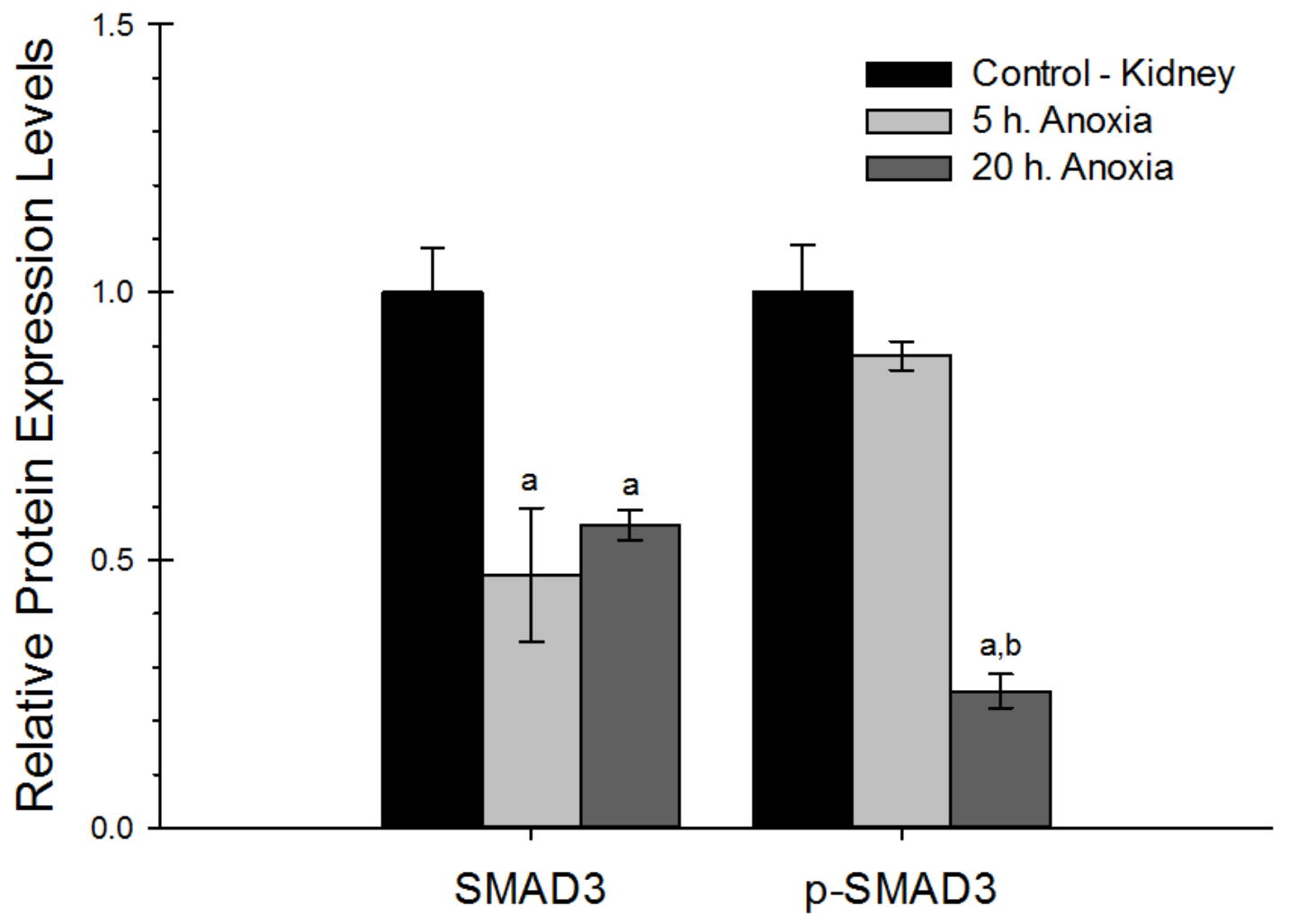

\section{B)}

\section{SMAD3}

p-SMAD3
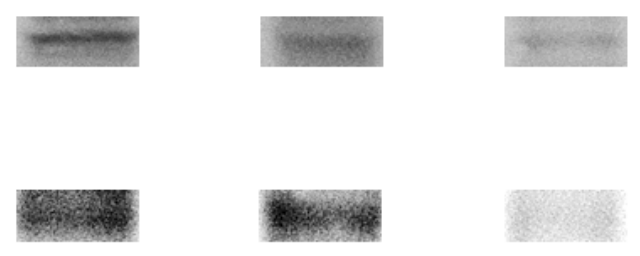

Figure 2.18 - Effects of five hour and twenty hour anoxic exposure on the protein expression of SMAD3 (48 kDa) and phospho-SMAD3 (serine 423/425) in red-eared slider kidney as determined by immunoblotting. A histogram of normalized mean protein levels (A) is shown along with representative immunoblots (B). Data are means \pm S.E.M., $n=3-4$ independent trials. Significantly different from the corresponding $(a)$ control or $(b) 5$ hour anoxia values using a one-way ANOVA and a Tukey's post-hoc test, $p<0.05$. 
A)

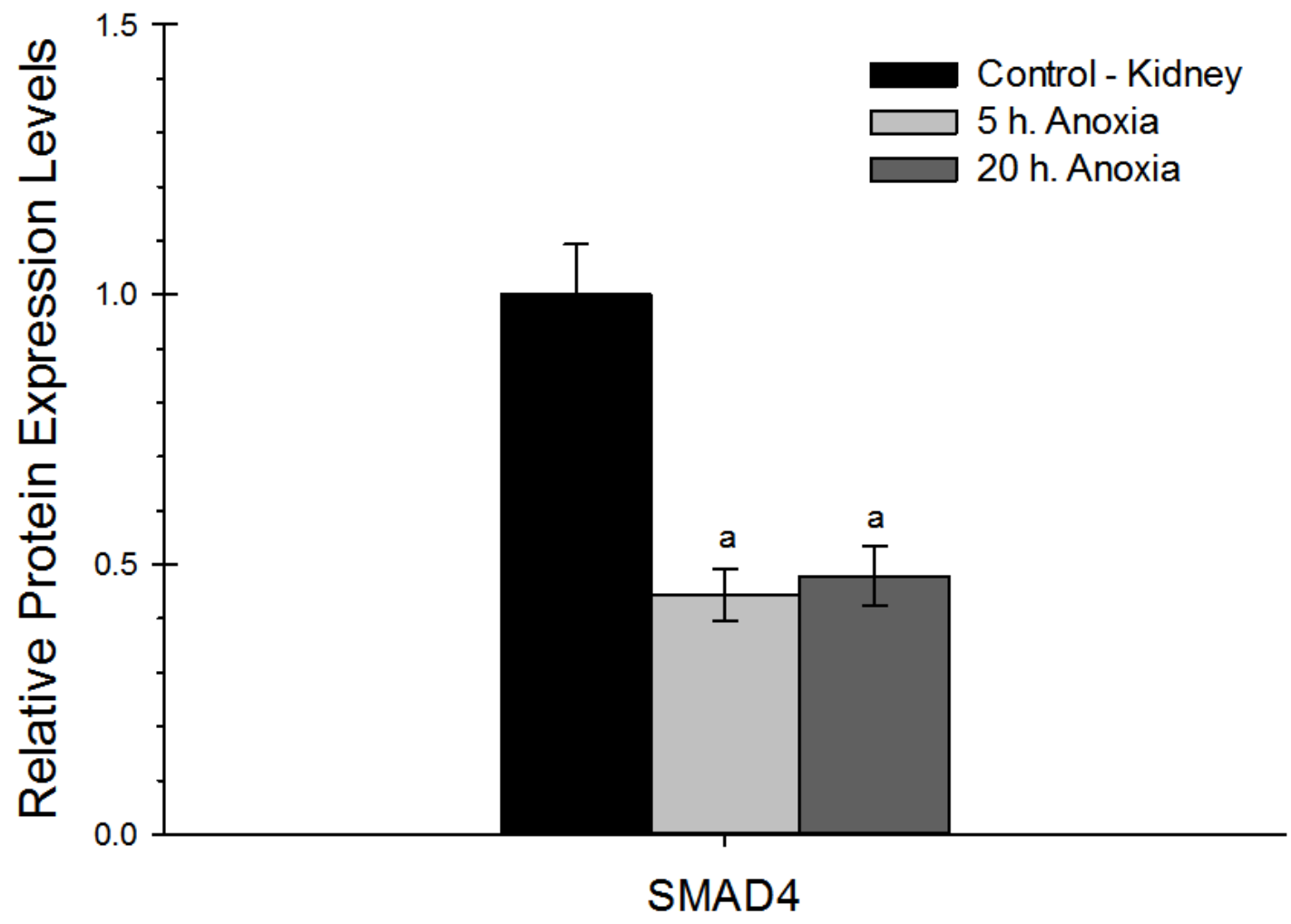

B)

\section{SMAD4}

Figure 2.19 - Effects of five hour and twenty hour anoxic exposure on the protein expression of SMAD4 $(60 \mathrm{kDa})$ in red-eared slider kidney as determined by immunoblotting. A histogram of normalized mean protein levels (A) is shown along with representative immunoblots $(\mathbf{B})$. Data are means \pm S.E.M., $n=3-4$ independent trials. Significantly different from the corresponding $(a)$ control or $(b) 5$ hour anoxia values using a one-way ANOVA and a Tukey's post-hoc test, $p<0.05$. 
A)

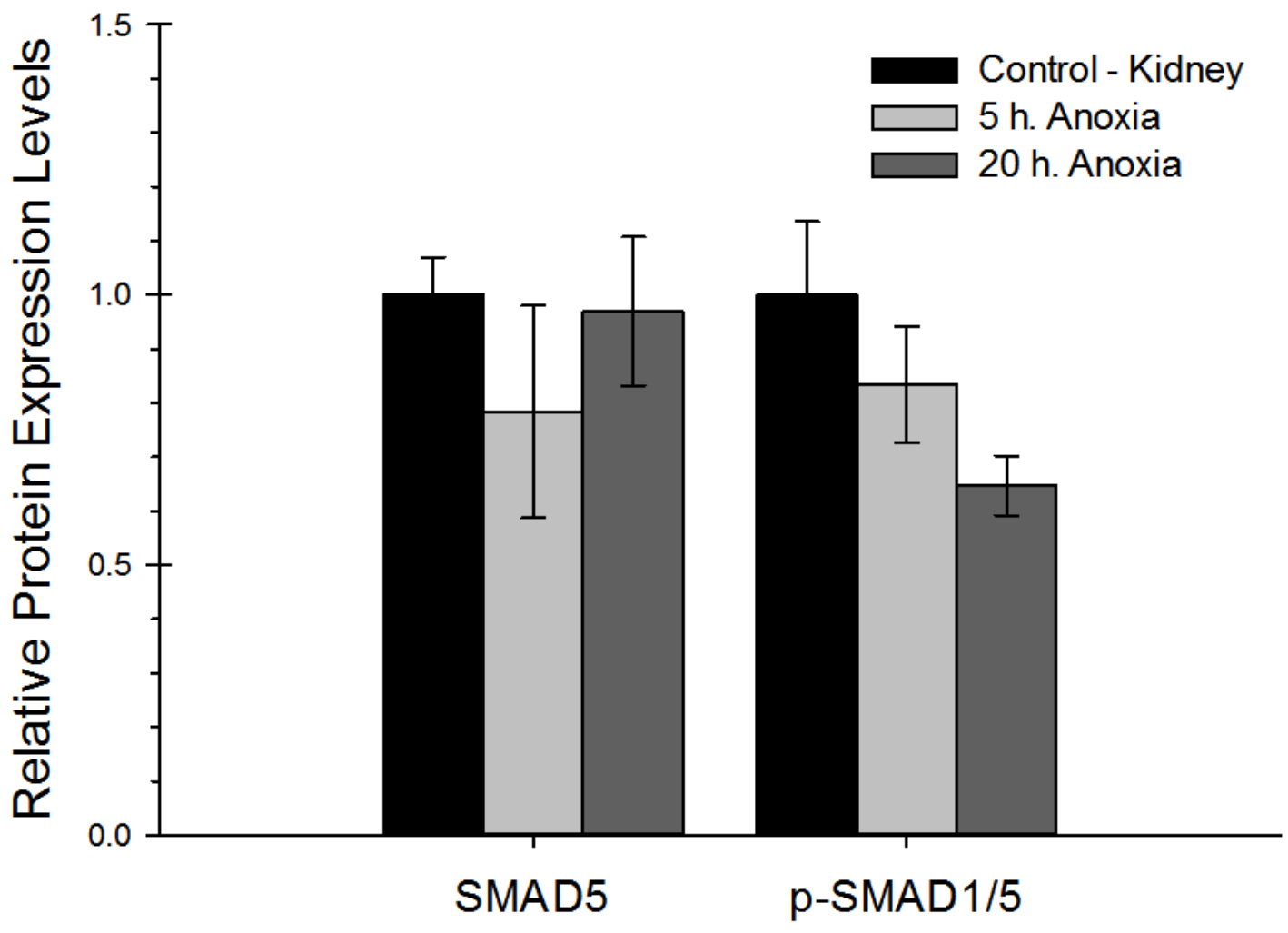

B)

\section{SMAD5}

p-SMAD1/5

Figure 2.20 - Effects of five hour and twenty hour anoxic exposure on the protein expression of SMAD5 (52 kDa) and phospho-SMAD1/5 (serine 463/465) in redeared slider kidney as determined by immunoblotting. A histogram of normalized mean protein levels $(\mathbf{A})$ is shown along with representative immunoblots (B). Data are means \pm S.E.M., $n=3-4$ independent trials. Significantly different from the corresponding $(a)$ control or $(b) 5$ hour anoxia values using a one-way ANOVA and a Tukey's post-hoc test, $p<0.05$. 


\subsection{5 - SMAD Heart Expression in Anoxic Conditions}

In heart, SMAD1 total protein expression levels were unchanged in 5 hour anoxic turtles, but increased to almost 1.6-fold higher than the control levels after 20 hour anoxia (5hA: $0.688 \pm 0.198$, N.S.; $20 \mathrm{hA}: 1.557 \pm 0.055, p<0.05)$ (Figure 2.21); there was also a significant difference between the levels of SMAD1 at 5 versus 20 hours of anoxia $(p<0.01)$. Its counterpart, SMAD5, also showed no change after 5 hours anoxia (5hA: 0.963 \pm 0.139, N.S.) but after 20 hour anoxia levels were only about a third of the control values (20hA: $0.324 \pm 0.056, p<0.005)$ (Figure 2.25); total protein levels again differed significantly between the two anoxic groups $(p<0.005)$. Phosphorylation of SMAD1/5 mimicked the pattern seen for SMAD5 with levels remaining at parity with the control levels at 5 hour anoxia (5hA: $0.940 \pm 0.083$, N.S.) and decreasing significantly after 20 hour anoxia (20hA: $0.644 \pm 0.023, p<0.05)$; phosphorylated protein levels also changed significantly between 5 and 20 hour anoxia $(p<0.05)$. SMAD2 normalized band intensities on the immunoblot indicated that its relative total protein expression increased substantially to $173 \%$ of the control level in low anoxic stress conditions, and remained high at $150 \%$ of the control value under extended anoxia stress conditions (5hA: $1.726 \pm 0.035, p<0.001$; 20hA: $1.499 \pm 0.072, p<0.01$ ) (Figure 2.22). However, the phosphorylated form of SMAD2 showed no significant changes relative to normoxic conditions at either 5 hour or 20 hour anoxia (5hA: $0.728 \pm 0.090$, N.S.; 20hA: $0.975 \pm 0.099$, N.S.). SMAD3 experienced an upregulation at $5 \mathrm{~h}$ anoxia and returned to normoxia levels at $20 \mathrm{~h}$ anoxia (5hA: $2.227 \pm$ $0.119, p<0.001 ; 20 \mathrm{hA}: 0.748 \pm 0.046$, N.S.) (Figure 2.23). There was a significant difference detected between both anoxic conditions, 5hA and 20hA $(p<0.001)$.Activated phosphorylated SMAD3 (p-SMAD3) did not change significantly after 5 hours of anoxia (5hA: $0.823 \pm 0.106, N . S$ ), but decreased strongly after 20 hours of anoxic stress to only 
one third of the normoxic protein expression level (20hA: $0.366 \pm 0.043, p<0.05)$ (Figure 2.23). The common SMAD4 showed a change only after 20 hours of anoxic exposure with its expression levels rising 1.81-fold relative to control heart tissues $(5 \mathrm{hA}: 0.950 \pm 0.149$, N.S.; $20 \mathrm{hA}: 1.813 \pm 0.111, p<0.005)$; this level was also significantly different from the 5 hour anoxia treatment group $(p<0.005)$ (Figure 2.24). 


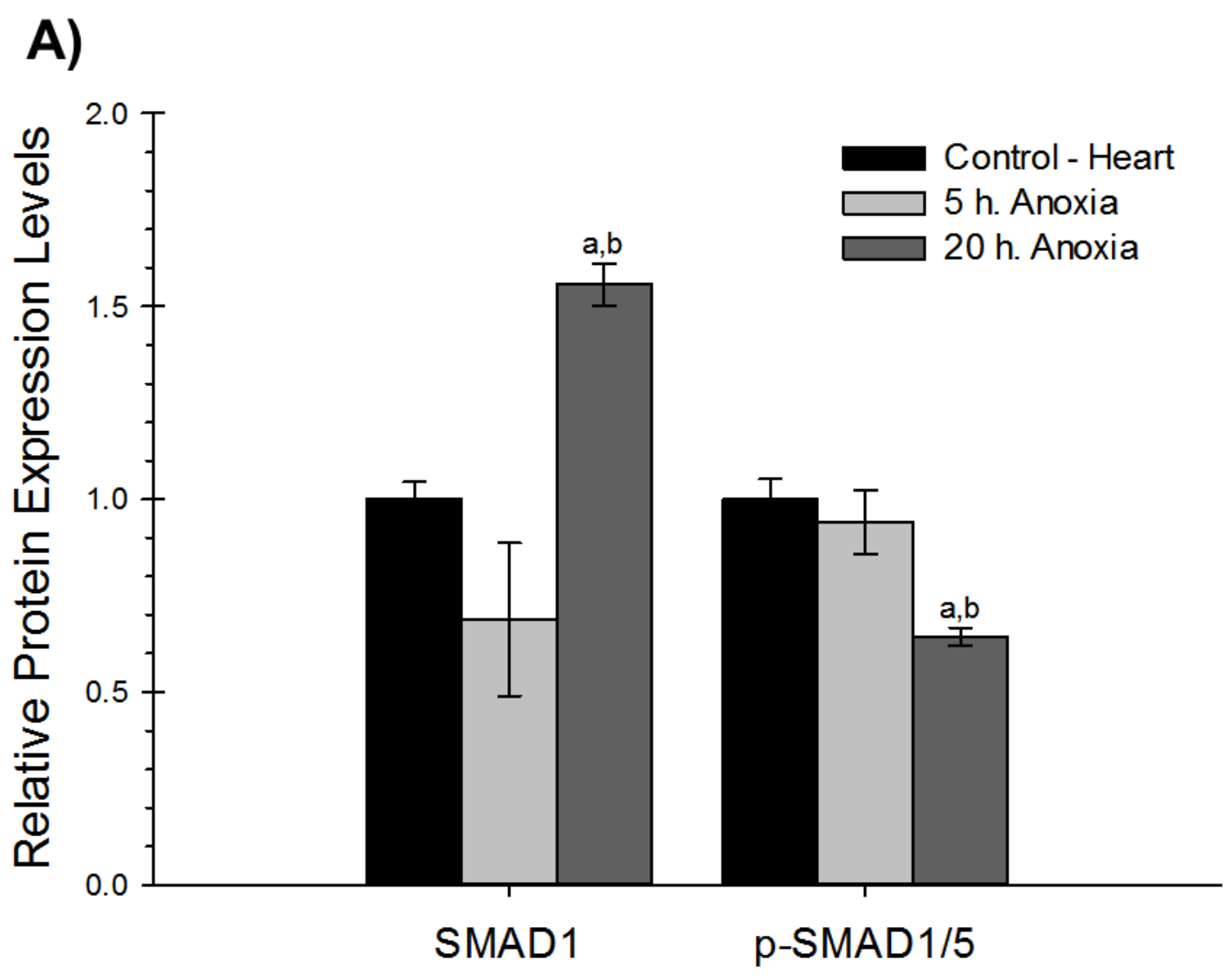

B)

\section{SMAD1}
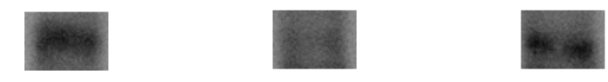

\section{p-SMAD1/5}

Figure 2.21 - Effects of five hour and twenty hour anoxic exposure on the protein expression of SMAD1 (52 kDa) and phospho-SMAD1/5 (serine 463/465) in redeared slider heart as determined by immunoblotting. A histogram of normalized mean protein levels $(\mathbf{A})$ is shown along with representative immunoblots (B). Data are means \pm S.E.M., $n=3-4$ independent trials. Significantly different from the corresponding $(a)$ control or $(b) 5$ hour anoxia values using a one-way ANOVA and a Tukey's post-hoc test, $p<0.05$. 
A)

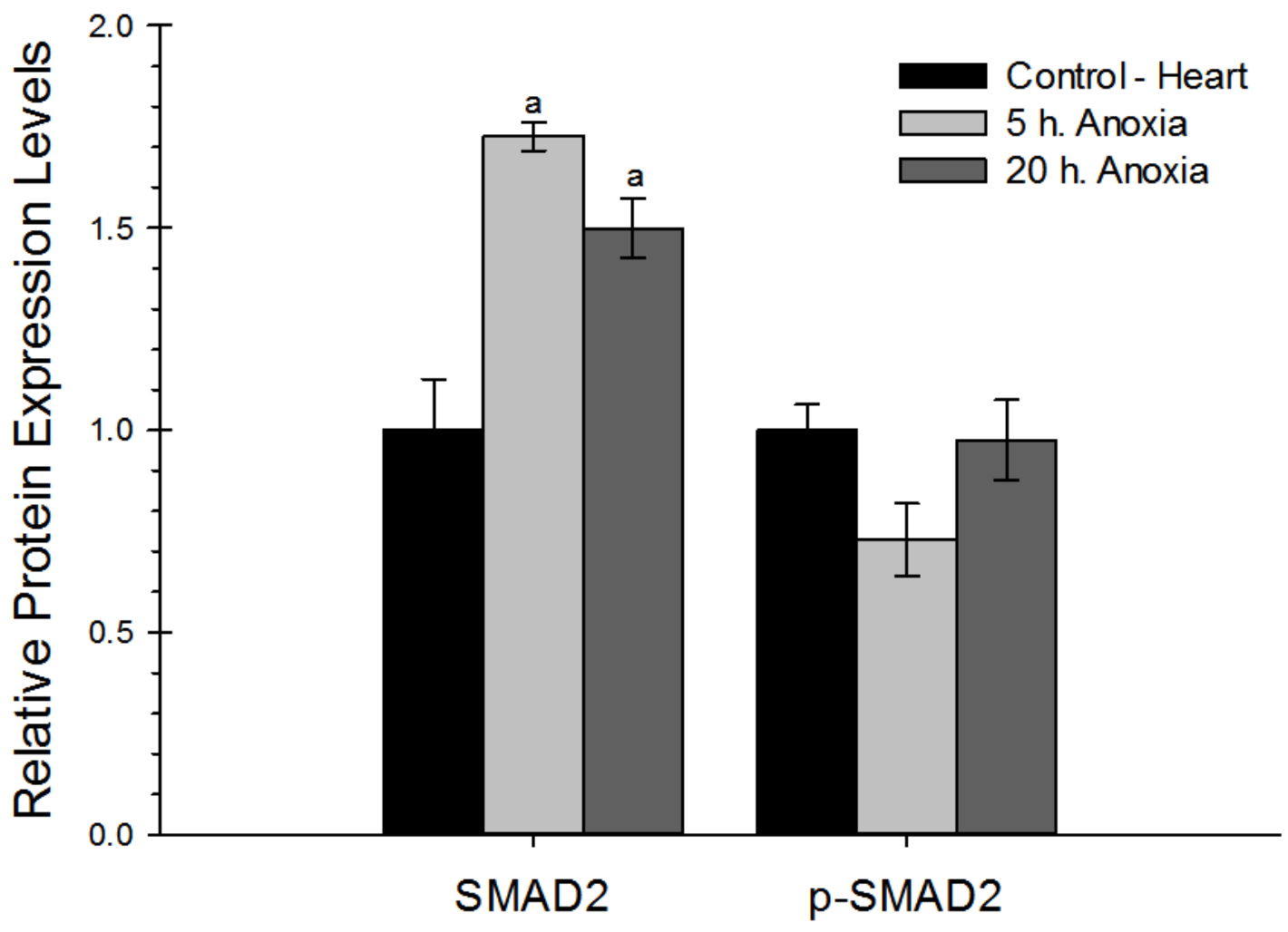

B)

SMAD2
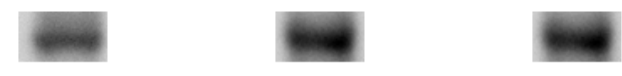

p-SMAD2
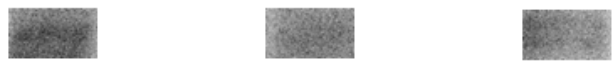

Figure 2.22 - Effects of five hour and twenty hour anoxic exposure on the protein expression of SMAD2 (52 kDa) and phospho-SMAD2 (serine 465/467) in red-eared slider heart as determined by immunoblotting. A histogram of normalized mean protein levels (A) is shown along with representative immunoblots (B). Data are means \pm S.E.M., $n=3-4$ independent trials. Significantly different from the corresponding $(a)$ control or $(b) 5$ hour anoxia values using a one-way ANOVA and a Tukey's post-hoc test, $p<0.05$. 


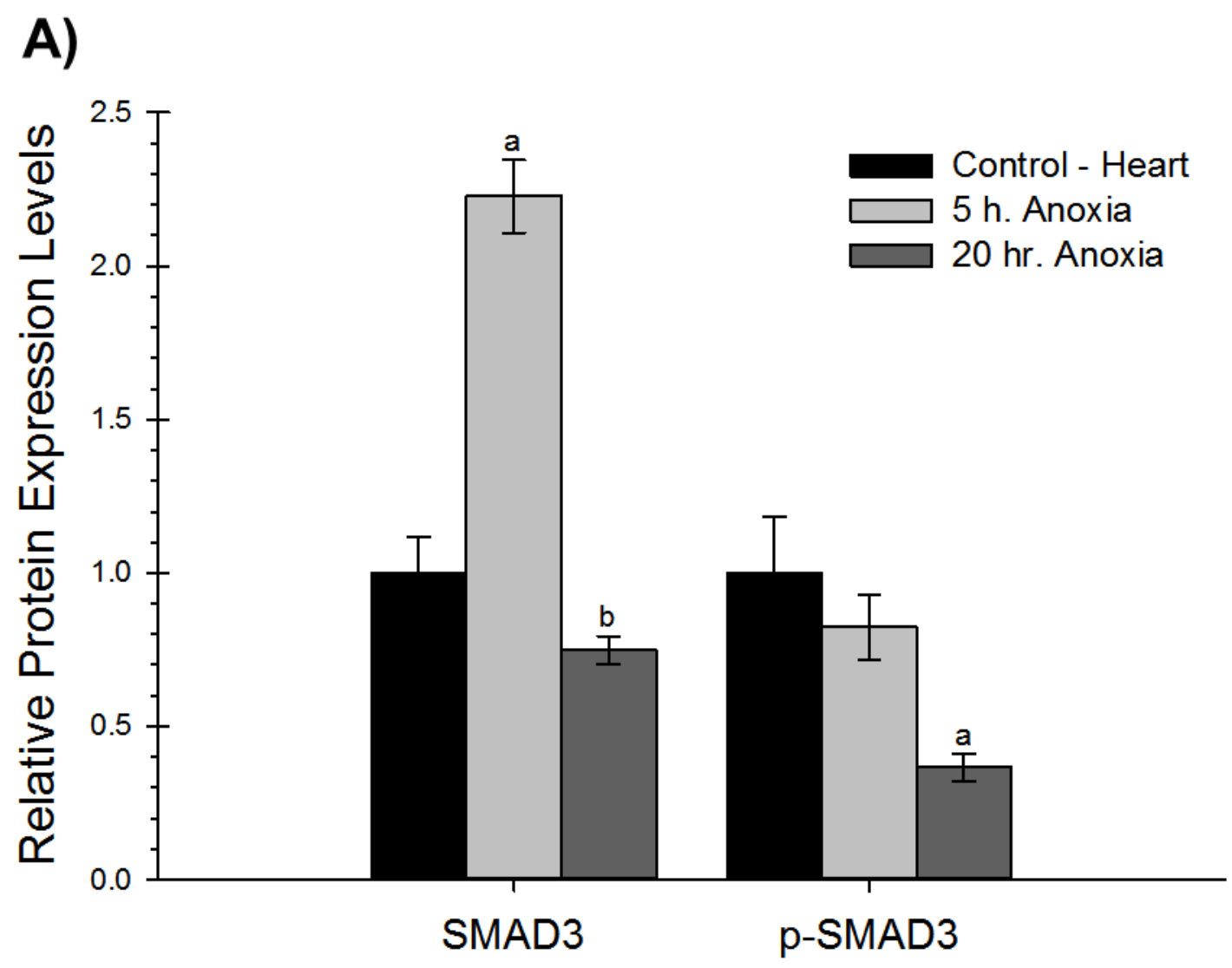

B)

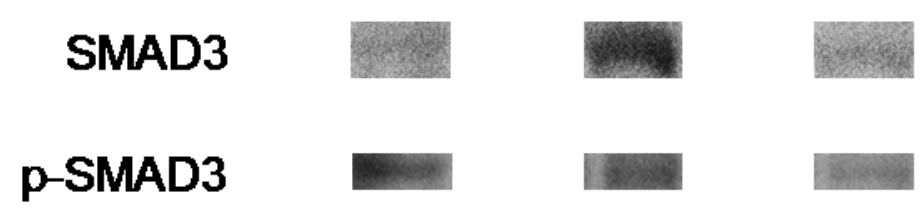

Figure 2.23 - Effects of five hour and twenty hour anoxic exposure on the protein expression of SMAD3 (48 kDa) and phospho-SMAD3 (serine 423/425) in red-eared slider heart as determined by immunoblotting. A histogram of normalized mean protein levels (A) is shown along with representative immunoblots (B). Data are means \pm S.E.M., $n=3-4$ independent trials. Significantly different from the corresponding $(a)$ control or $(b) 5$ hour anoxia values using a one-way ANOVA and a Tukey's post-hoc test, $p<0.05$. 


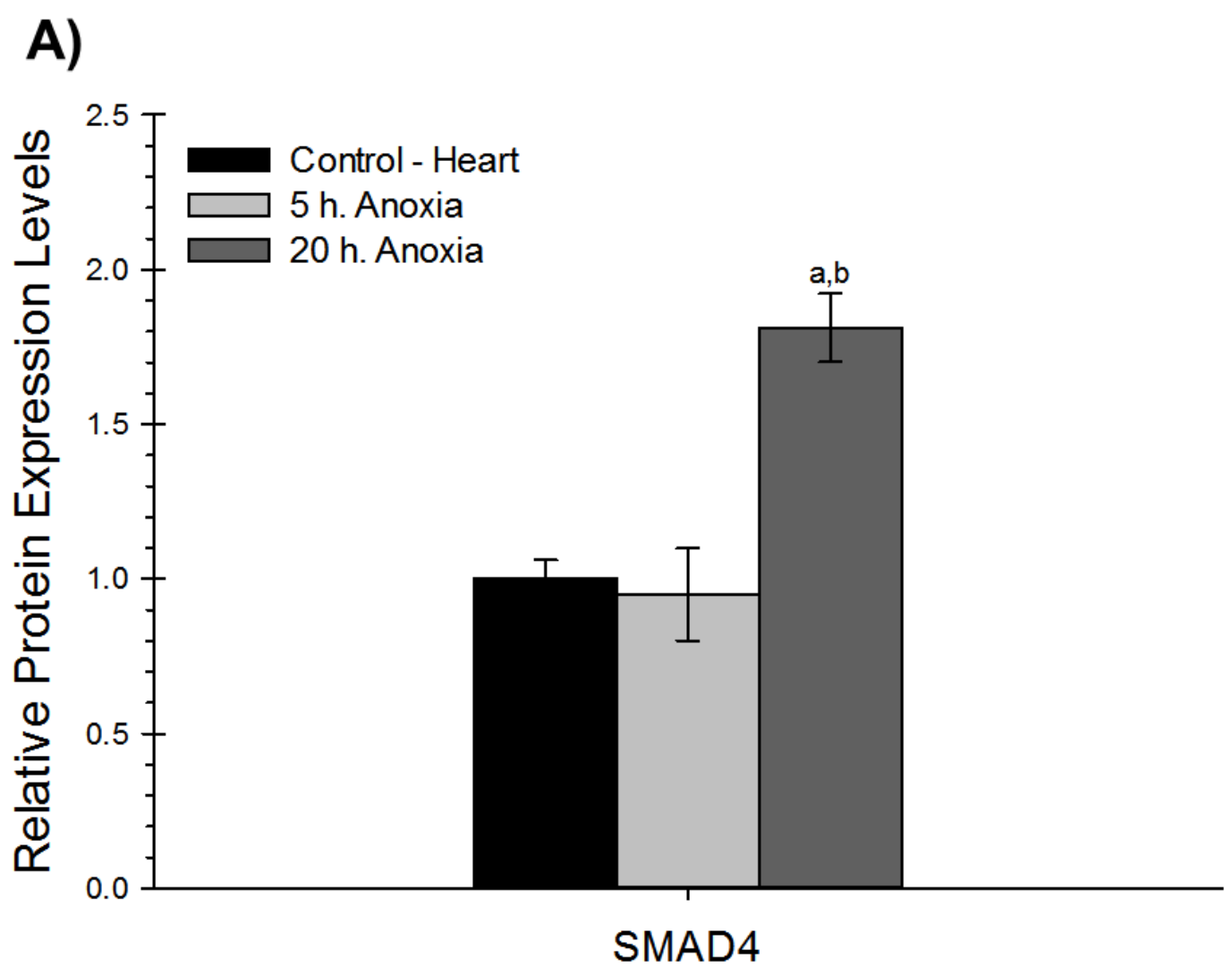

B)

SMAD4

Figure 2.24 - Effects of five hour and twenty hour anoxic exposure on the protein expression of SMAD4 $(60 \mathrm{kDa})$ in red-eared slider heart as determined by immunoblotting. A histogram of normalized mean protein levels (A) is shown along with representative immunoblots (B). Data are means \pm S.E.M., $n=3-4$ independent trials. Significantly different from the corresponding $(a)$ control or $(b) 5$ hour anoxia values using a one-way ANOVA and a Tukey's post-hoc test, $p<0.05$. 


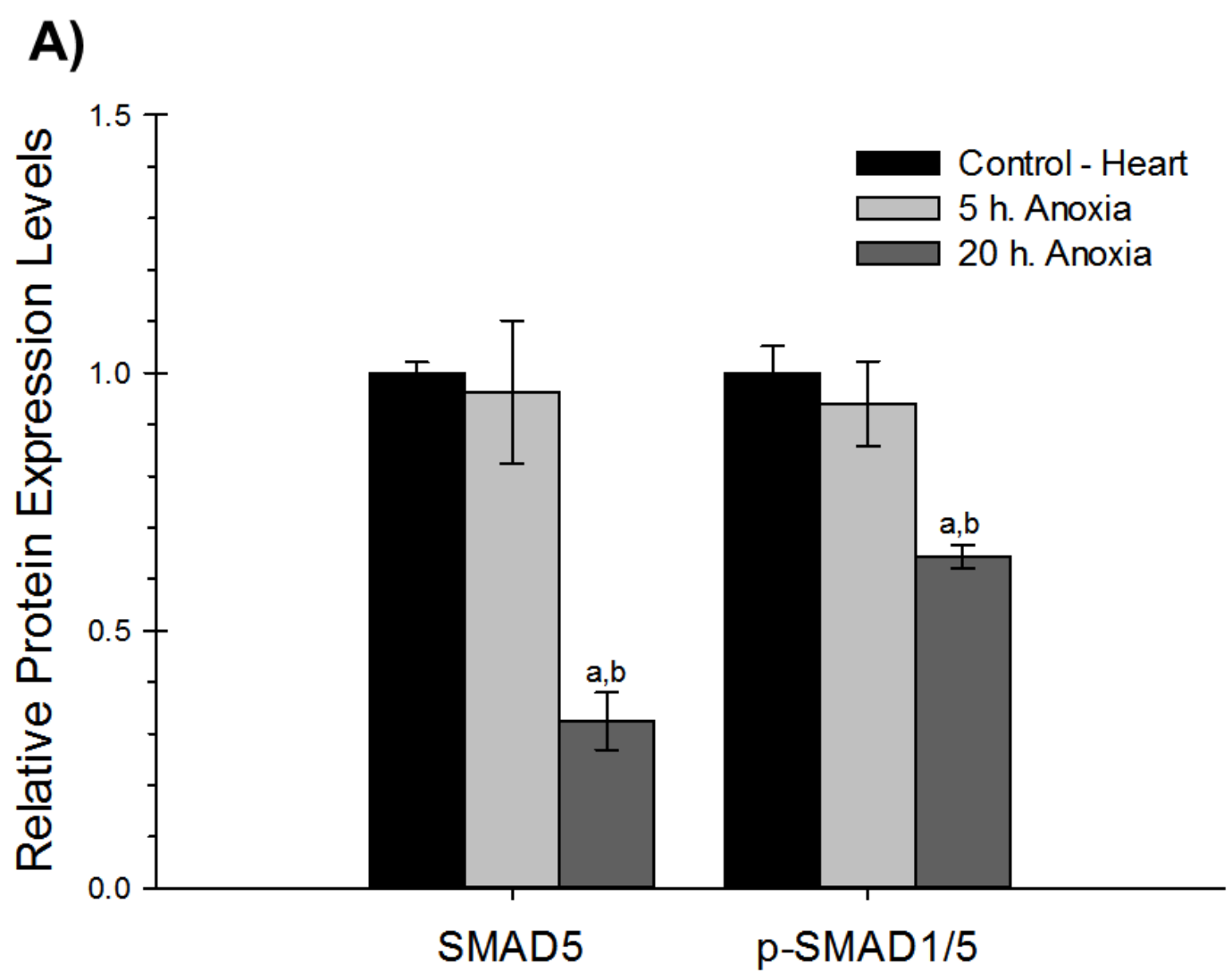

B)

\section{SMAD5}
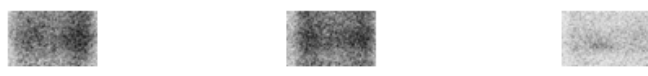

\section{p-SMAD1/5}

Figure 2.25 - Effects of five hour and twenty hour anoxic exposure on the protein expression of SMAD5 (52 kDa) and phospho-SMAD1/5 (serine 463/465) in red-eared slider heart as determined by immunoblotting. A histogram of normalized mean protein levels $(\mathbf{A})$ is shown along with representative immunoblots $(\mathbf{B})$. Data are means \pm S.E.M., $n=3-4$ independent trials. Significantly different from the corresponding (a) control or $(b) 5$ hour anoxia values using a one-way ANOVA and a Tukey's post-hoc test, $p<0.05$. 


\subsection{6 - TGF- $\beta$ Superfamily Up- and Downstream Protein Expression}

Relative levels of the TGF- $\beta$ precursor protein were measured in liver, white muscle, red muscle, kidney and heart (Figure 2.26). In liver, TGF- $\beta$ precursor levels showed a significant reduction after 5 hours of anoxia exposure but values were restored to near control levels after 20 hours anoxia (5hA: $0.386 \pm 0.018, p<0.001 ; 20 \mathrm{hA}: 0.916 \pm 0.12$, N.S.). It is worthwhile to note the two anoxic conditions were significantly different from each other as well $(p<0.001)$. In white muscle the TGF- $\beta$ precursor protein levels remained virtually constant after 5 hour anoxia, but by 20 hours of anoxia the levels had plummeted to just $5.7 \%$ of the original control values (5hA: $1.131 \pm 0.151$, N.S.; $20 \mathrm{hA}: 0.0570 \pm$ $0.0225, p<0.005)$; this level also significantly differed from the 5 hour anoxic level $(p<0.001)$. Expression levels of TGF- $\beta$ precursor protein did not change significantly in red skeletal muscle after either 5 or 20 hours anoxia (5hA: $1.059 \pm 0.162$, N.S.; 20hA: 1.039 $\pm 0.280, N . S$.). In $T$. scripta elegans kidney, the expression of TGF- $\beta$ precursor protein rose 1.38-fold relative to controls after 5 hours of anoxic stress, but the difference was not significant compared to controls (5hA: $1.377 \pm 0.200$, N.S.); however, after $20 \mathrm{~h}$ anoxia (20hA: $0.0311 \pm 0.0128)$ levels fell to very low values as compared with controls $(p<0.005)$ or 5 hours anoxia $(p<0.001)$. In heart, TGF- $\beta$ precursor levels decreased significantly after 5 hours of anoxic stress and subsequently rose by 1.69 -fold relative to normoxic heart after 20 hour anoxia (5hA: $0.507 \pm 0.065, p<0.05 ; 20 \mathrm{hA}: 1.694 \pm 0.174, p<0.01)$ and were also significantly different from the 5 hour anoxia group $(p<0.001)$.

The effect of anoxia exposure on total and phosphorylated c-Myc expression was also examined in turtle tissues. In red skeletal muscle total c-Myc levels were unchanged from control to $5 \mathrm{~h}$ anoxic turtles and became suppressed at $20 \mathrm{~h}$ anoxia to $35 \%$ relative 
expression to control (5hA: $0.828 \pm 0.180$, N.S.; $20 \mathrm{hA}: 0.348 \pm 0.088, p<0.05)$ (Figure 2.27). Phospho-c-Myc levels meanwhile remained at parity with controls during $5 \mathrm{~h}$ anoxia, and subsequently was upregulated 1.52 -fold relative CTSE during 20h anoxia, however this difference was deemed non-significant by ANOVA testing (5hA: $1.001 \pm 0.159$, N.S.; 20hA: $1.524 \pm 0.310, N . S$. 
A)

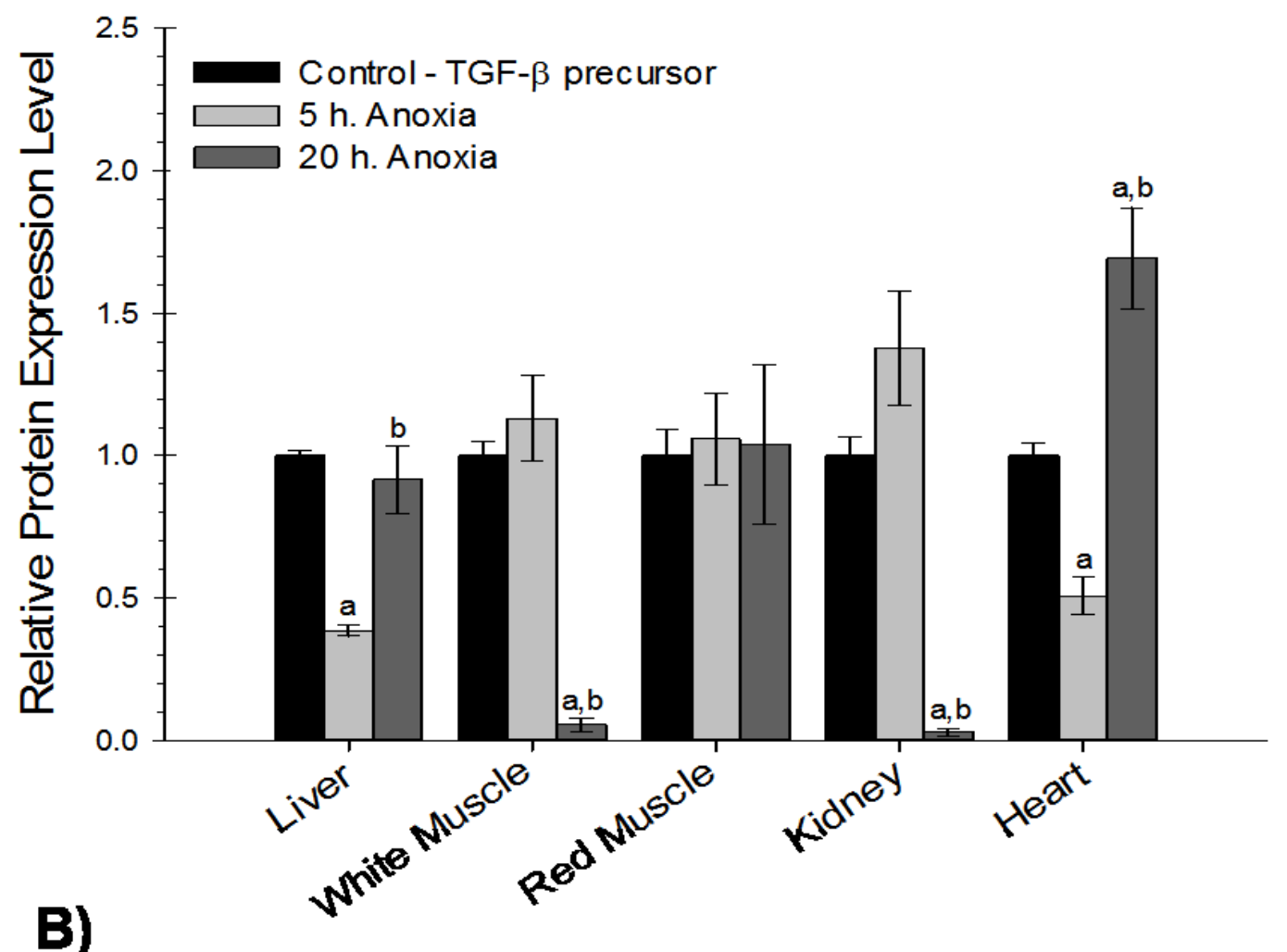

Liver
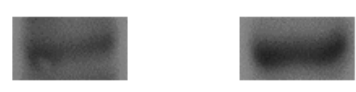

White Muscle
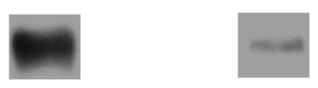

Red Muscle

Kidney
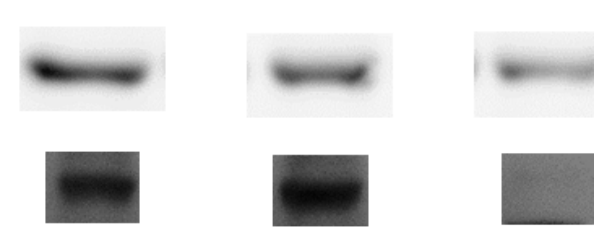

\section{Heart}

Figure 2.26 - Effects of five hour and twenty hour anoxic exposure on the protein expression of TGF- $\beta$ precursor (45 $\mathrm{kDa}$ ) in red-eared slider liver, white muscle, red muscle, kidney, and heart as determined by immunoblotting. A histogram of normalized mean protein levels (A) is shown along with representative immunoblots (B). Data are means \pm S.E.M., $n=3-4$ independent trials. Significantly different from the corresponding $(a)$ control or $(b) 5$ hour anoxia values using a one-way ANOVA and a Tukey's post-hoc test, $p<0.05$. 
A)

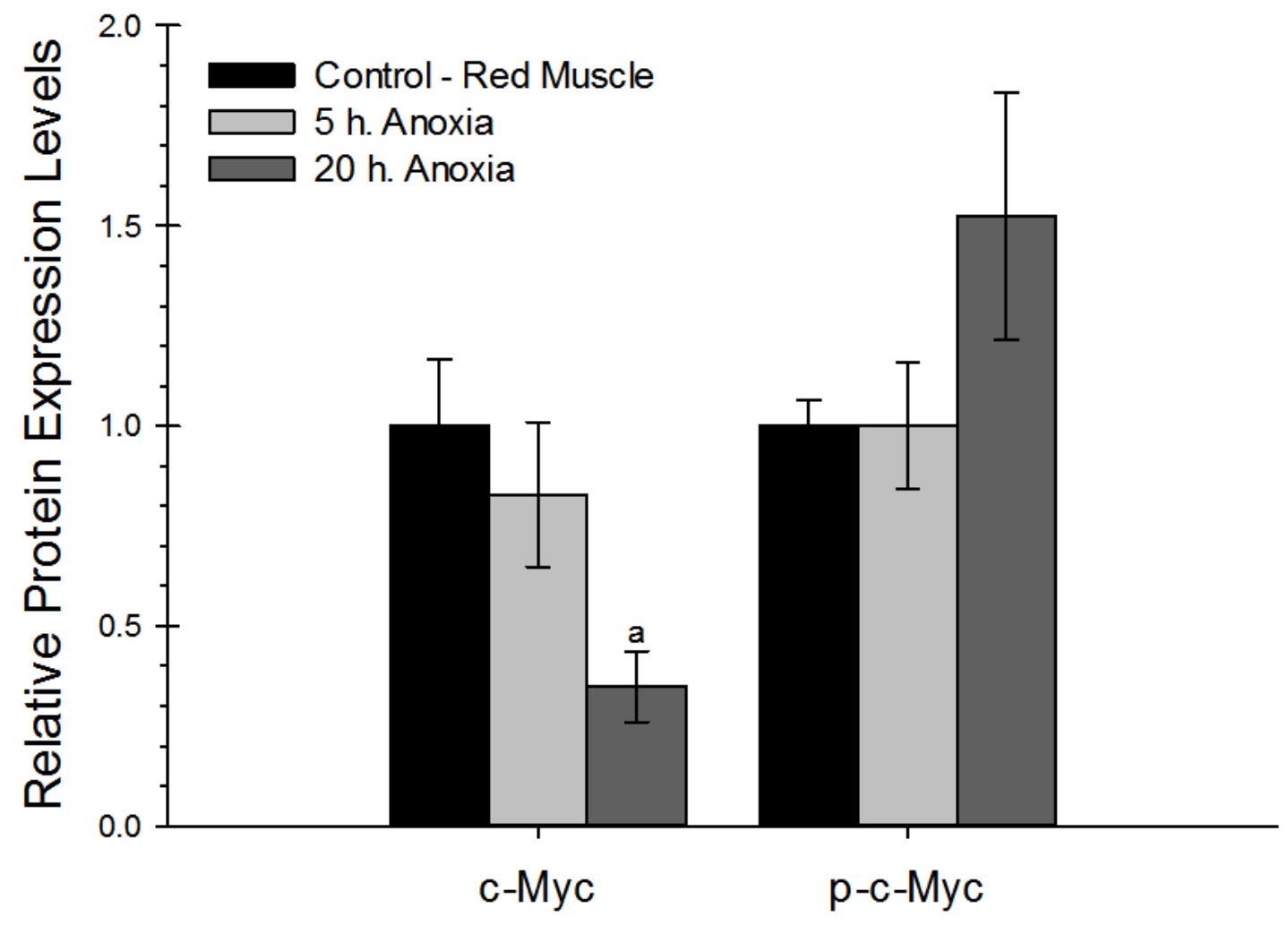

B)

\section{c-Myc}

\section{p-c-Myc}

Figure 2.27 - Effects of five hour and twenty hour anoxic exposure on the protein expression of total c-Myc $(47 \mathrm{kDa})$ and phospho-c-Myc (threonine 58/serine 62$)$ in red-eared slider red muscle as determined by immunoblotting Representative immunoblots are shown along with a histogram showing normalized mean protein levels. Data are means \pm S.E.M., $n=3-4$ independent trials. Significantly different from the corresponding ${ }^{\mathrm{a}}$ control or ${ }^{\mathrm{b}} 5$ hour anoxia values using a one-way ANOVA and a Tukey's post-hoc test, $p<0.05$. 


\section{4 - Discussion}

As the winter progresses, it becomes harder and harder for turtles to survive. This is due to the lack of oxygen available to them in their aquatic environs; ice cover makes it impossible for turtles to surface to breathe with lungs and the ability to take up oxygen by nonpulmonary mechanisms declines as oxygen is used up by all the species living in the same body of water. To alleviate this problem turtles have adopted several survival mechanisms. Pathways involved in ATP-expensive activities such as growth, proliferation, and protein synthesis are the first to be suppressed in a survival strategy known as hypometabolism. It has been shown by numerous studies that hypoxia/anoxia (via the transcription factor HIF-1 $\alpha$ ) induces a $\mathrm{G}_{1}$ arrest which is associated with a decreased activity of some cyclin-CDK complexes (Goda, et al., 2003; Lando et al., 2002; Schipani et al.; 2001), while mTor (largely responsible for protein synthesis) is also shut down as a consequence of anoxia (Wouters and Koritzinsky, 2008; Vadysirisack and Ellisen, 2012; Brugarolas et al., 2004). This kind of response is typically mediated by cytokines, first and second messengers, and other signaling molecules created endogenously. In this study, I focused on the TGF- $\beta$ and BMP families of cytokines and their associated signaling transcription factors as well as the proteins that are affected downstream of the TGF- $\beta$ signaling pathway.

As mentioned in Chapter 1, TGF- $\beta$ signaling is initiated by the accompanying cytokines of the same name. TGF- $\beta 1$ is a homodimer of two $11 \mathrm{kDa}$ polypeptides that are 112 amino acids in length. The dimer is held together by disulphide bonds that are formed by its characteristic pattern of nine cysteines. Two other TGF- $\beta$ proteins have also been discovered and are aptly named TGF- $\beta 2$ and TGF- $\beta 3$. All three TGF- $\beta$ s are initially 
synthesized in precursor form with two large prosegment molecules that interact noncovalently with the mature C-terminal TGF- $\beta$ dimer and maintain it in a biologically inactive form. In this form it is unable to bind to the receptors and must be cleaved from the C-terminal mature monomer in order for it to be active (Figure 2.28) (Derynck and Feng, 1997, Kretzschmar and Massagué, 1998, Feng and Derynck, 2005, Kang et al., 2009).

\subsection{1 - TGF- $\beta$ Superfamily Mediated Effects in TSE Liver}

\subsubsection{1 - Cell Cycle Regulation}

In the liver of the anoxia-tolerant freshwater turtle, the precursor of the TGF- $\beta$ I-III cytokines had a lower expression level than controls during 5h anoxia (Figure 2.26). This observation could mean that R-SMADs 2 and 3, as well as their respective phosphorylation levels, should show the same downregulation because their recruitment to the receptor for phosphorylation is proportional to the cytokine signal. Indeed, SMAD2 and p-SMAD2 were suppressed during 20h anoxia (Figure 2.2), whereas SMAD3 and p-SMAD3 both dropped after $5 \mathrm{~h}$ anoxic exposure and were ultimately maintained at about $40 \%$ of their respective control levels at 20h anoxia (Figure 2.3). These results can be attributed and are in agreement with previous conclusions that indicate that liver enters into a cell cycle $\mathrm{G}_{1}$ (gap phase 1) arrest during anoxia (Biggar, 2013). Additionally, the responses of SMAD4 back up this conclusion since its expression levels dropped dramatically to below 50\% relative to controls at $5 \mathrm{~h}$ anoxia and fell further to between 10 and $20 \%$ of controls after enduring 20h anoxic stress (Figure 2.4). The link between these SMADs in hepatocytes and those regulating the cell cycle is well understood and documented (Weinstein et al., 2001; Kretschmer et al., 2003; Dzieran et al., 2013). The literature indicates that, while 
phosphorylated, SMADs 2 and 3 bind to $C D K N 1 A$ promoter regions in the DNA and help to stimulate p21 expression in the cell (Figure 2.29) (Massagué, 2012). This effect was indeed observed in turtle liver where the phosphorylated levels of p21 (1.6-fold change in $5 \mathrm{~h}$ and threefold change in $20 \mathrm{~h}$ anoxia) and total levels of another cell cycle inhibitor, p27 (1.2-fold change in $5 \mathrm{~h}$ and threefold change in $20 \mathrm{~h}$ anoxia) were seen to increase under $20 \mathrm{~h}$ anoxia confirming that cell cycle arrest is indeed occurring (Biggar and Storey, 2009). In turtle liver c-Myc, an inhibitor of p21 was suppressed (Figure 2.27), so its expression levels under anoxia were in agreement with the cell cycle arrest concept (Biggar, 2013). Once cell growth is stopped the cell does not require multiple inhibitors to be upregulated as this is energetically unfavourable. Following $20 \mathrm{~h}$ anoxia, phosphorylated p 27 was in fact downregulated, however cell cycle arrest is maintained as the expression levels of other CDK inhibitors are sustained such as p16 whose expression is increased at 5 hours of anoxia and maintained in an elevated state during 20h anoxia (Biggar, 2013).

\subsubsection{2 - Angiogenesis Regulation}

Just as the cell cycle is halted as a result of anoxia and the consequential deficiency of ATP equivalents, angiogenesis is another process that the turtle could regulate in order to survive and maintain an equilibrium between energy consumed and energy generated. This equilibrium is known as hypometabolism and forms the basis of the majority of fates of molecular pathways in the cell when faced with extremely unfavourable living conditions, which may otherwise render the organism severely incapacitated or even lifeless. SMAD5 was upregulated 1.9-fold in the liver during 20h anoxia (Figure 2.5), and in contrast SMAD1 was downregulated under both anoxic conditions along with its phosphorylated form (Figure 2.1). These SMAD proteins control and promote the angiogenesis and 
vasculogenesis in the body of the red-eared slider. Angiogenesis refers to the creation of vessels from pre-existing ones, whereas vasculogenesis is the de novo formation of blood vessels by changing endothelial cells (EC) into angioblasts and typically precedes angiogenesis (Pardali et al., 2010; Weis and Cheresh, 2011). TGF- $\beta$ and other growth factors (VEGF and FGF) promote the development of smooth muscle cells (SMC) surrounding a vessel to stabilize and support it (vessel muscularization) through an important process to tissue regeneration known as Endothelial-to-Mesenchymal Transition (EndMT) (ten Dijke and Arthur, 2007; van Meeteren and ten Dijke, 2012. Massagué, 2012) (Figure 2.32). Typically, growth factors stimulate ECs surrounding the vessels and promote their transition into so-called tip cells (mesenchymal cells) which then are manipulated and guided by macrophages before fusing together and creating new vascular structures (Yancopoulos et al., 2000; Schmidt and Carmeliet, 2010; ten Dijke and Arthur, 2007; Fantin et al., 2010). TGF- $\beta$ and SMAD3, in conjunction, can affect the PI3K/Akt signal transduction pathway (a known mediator of cell-cycle progression and cell proliferation) (Figure 2.31) in vascular SMCs (Suwanabol et al., 2012). Additionally, SMADs 1 and 5 convey cytokine growth signals into the nucleus and bind to the SERPINE1 gene with the aid of AP-1 transcription factor complexes (c-Fos and c-Jun dimers) which codes and expresses its associated protein, PAI-1 (Liberati et al., 1999; ten Dijke and Arthur, 2007). Hypoxia, along with local nutritional deficiencies, has been shown to be a sort of angiogenic switch (Weis and Cheresh, 2011). However, the simultaneous suppression of both SMAD1 and phospho-SMAD1/5 could indicate that the liver is angiostatic rather than participating in angiogenesis when under anoxic conditions. The concomitant suppression of c-Fos at $20 \mathrm{~h}$ anoxic stress also provides evidence of this as it 
is essential in allowing SMADs to bind to the SERPINE1 gene (Greenway and Storey, 2000; Massagué, 2012) (Figure 2.29). This slowing of angiogenic activity may have an added survival advantage of reducing the number of fibroblast cells in circulation as the turtle would not require wound healing while dormant in a lake or pond devoid of oxygen, and these cells exhibit activated metabolism like other stem cells (Ghesquière et al., 2014; Lemons et al., 2010). It would seem like a contradiction then that c-Jun (the second protein in the AP-1 dimer complexes) is upregulated in the turtle after $5 \mathrm{~h}$ anoxia, but this is possibly due to its alternate role in protecting the cell from oxidative injury (Greenway and Storey, 2000).

\subsubsection{3 - ROS Defense Regulation}

As mentioned above in 2.4.1.2, c-Jun is upregulated and cannot be explained by the reduction in angiogenesis occurring within the liver of the turtle experiencing short-term anoxia. As anoxia progresses there can be an increase in the net level of reactive oxygen species (ROS) in tissues as a result of altered rates of ROS production versus destruction. One sign of this is the $50 \%$ depletion of glutathione, a key antioxidant metabolite in cells, in response to $20 \mathrm{~h}$ anoxic submergence (Willmore and Storey, 1997). As the amount of ROS increases residues that comprise proteins are affected and can become oxidized affecting their function. AP-1 proteins (Fos and Jun) are an example of such proteins that have their DNA binding ability neutralized through the oxidation of the cysteine residues that are embedded in the sequence involved in AP-1 DNA binding (Blokhina, et al., 2003; Hansen et al., 2006). However, the hypoxia-induced physiological defense mounted by the turtle is similar to the frog's response induced by dehydration at the molecular level. Phosphorylated c-Myc protein in the liver is upregulated under both anoxic conditions, just 
as it is in frog liver (Biggar, 2013). This phosphorylation has been shown to activate and create known redox molecules within the cell through the transcription of $\gamma$-glutamyl cysteine synthetase ( $\gamma$-GCS) (Benassi et al., 2006). ROS, such as $\mathrm{H}_{2} \mathrm{O}_{2}$, enhances and augments the recruitment of phosphorylated c-Myc to $\gamma$-GCS promoter sites and commences the cell's response to oxidative stress (Benassi et al., 2006). Responses by the forkhead box $\mathrm{O}$ transcription factors (FoxO1 and $\mathrm{FoxO} 3$ ) to anoxia have also been explored in turtle liver (Krivoruchko and Storey, 2013). FoxO3 was activated in the liver in response to anoxia as well as FoxO1 under anoxic conditions, as evidenced by increased expression, decreased phosphorylation (which is associated with inhibition of FoxOs), increased nuclear translocation, and increased DNA-binding activity. In addition, catalase was elevated in the liver in response to anoxia suggesting that FoxOs could play a role in the up-regulation of stress responsive antioxidant enzymes in turtles during anaerobiosis.

\section{$\underline{2.4 .2}$ - TGF- $\beta$ Superfamily Mediated Effects in TSE White Muscle}

\subsubsection{1-Cell Cycle Regulation}

During the winter months $T$. scripta elegans remains submerged and largely inactive. White muscle is known for its fast contraction time and contraction speeds by its fibers (Joanisse, 2004). This tissue is implicated in the "fight or flight" role as it uses high velocities of contraction and rapid energy production largely encompassing glycolytic metabolism, leading to the tissue being called the glycolytic muscle. It is with these details that I begin to explain my findings in the context of cell cycle arrest, or the lack thereof in white muscle. SMAD3 and its phosphorylated analog were observed to be modestly suppressed after $5 \mathrm{~h}$ anoxia (about $80 \%$ of control expression), while the total SMAD3 is again only modestly suppressed during 20 hour anoxia ( $80 \%$ of control) (Figure 2.8 ). 
Meanwhile SMAD2, the other R-SMAD isoform associated with growth and proliferation, was initially suppressed during $5 \mathrm{~h}$ anoxia, then contrastingly upregulated during 20 hour anoxia along with its phosphorylated form (about 1.5-fold of controls and 1.3-fold of controls, respectively) compared to other R-SMAD isoforms in white muscle (Figure 2.7). These results conflict and do not allow for the conclusion that white muscle is experiencing cell cycle arrest. Previous studies confirm that markers of cell cycle activity in turtle white skeletal muscle are minimally affected in response to oxygen deprivation. p21, c-Myc, and other CDK inhibitors were expressed at control levels throughout anoxia (Biggar, 2013). Interestingly, phospho-p21 was suppressed after 20h anoxia suggesting again that cell cycle arrest does not occur. The evidence in white skeletal muscle of the turtle suggests that cell cycle is minimally regulated during anoxia; likely a reflection of its overall postmitotic nature.

\subsubsection{2 - Angiogenesis Regulation}

BMP controlled R-SMADs 1 and 5 are known to play considerable roles in turning on or off angiogenesis (González-Núñez et al., 2013; Pardali et al., 2010). Total SMAD1 levels were found to decrease during $5 \mathrm{~h}$ anoxia, and total SMAD5 expression was suppressed in white muscle during 20h anoxia (Figures 2.6 and 2.10). This pattern is similar to the FoxO expression patterns in the same tissue seen in 2.4.2.3. Again, in this way, cells may conserve energy by using proteins that are pre-existing as this avoids the energy expensive process of protein synthesis, namely through translation. AP-1 proteins showed no significant differences in response to anoxia, indicating no regulation at the SMAD DNA-binding level - specifically to motifs that promote the expression of angiogenic proteins such as PAI-1. However, the subcellular localization of c-Fos is 
significantly lower in the nucleus further suggesting that AP-1 proteins are not being used to enhance SMAD complex-DNA binding. TGF- $\beta$ precursor protein expression were strongly suppressed at $20 \mathrm{~h}$ anoxia (less than $10 \%$ of control levels, Figure 2.26) and the common SMAD4 protein levels were also decreased after 5h anoxic stress supporting the observation of global suppression of R-SMADs, except in the case of SMAD2 and its phosphorylated form.

\subsubsection{3 - Oxidative Stress Response}

As white skeletal muscle is known to show basal metabolism of the glycogen stores of the turtle while remaining inactive, there may be a minimal production/accumulation rate of free radicals in the tissue due to the lack of mitochondria found in these tissues (Joanisse, 2004). The expression of forkhead class O (FoxO) proteins (FoxO1 and FoxO3), known mediators of a cell's response to oxidative stress, have been studied previously to assess the extent to which the cells must battle ROS accumulation. Based on this, it appears that the turtle probably experiences an increase in ROS levels in its muscle when oxygen supply is depleted because there is an upregulation of FoxO1 proteins at $5 \mathrm{~h}$ anoxia, indicative of a need for enhanced antioxidant defenses but no changes were detected during $20 \mathrm{~h}$ anoxia (Krivoruchko and Storey, 2013). FoxO3 takes over at 20h anoxia with upregulation observed for total FoxO3, phospho-FoxO3 (serine 253), and phospho-FoxO3 (serines 318 and 321) in white muscle - a signaling pattern not seen in the liver tissues. c-Myc, which has been shown in this study to strongly affect turtle liver ROS defenses while phosphorylated, is not differentially phosphorylated in turtle white skeletal muscle, once again potentially signifying that oxidative injury is not as prevalent in white muscle relative to liver tissues. 


\section{$\underline{2.4 .3}$ - TGF- $\beta$ Superfamily Mediated Effects in TSE Red Muscle}

\subsubsection{1 - Cell Cycle Regulation}

Skeletal muscle possesses the ability to greatly adapt to its environment and function in relation to metabolism and size. Red muscle receives its moniker from the increased myoglobin content within the myofibers in comparison to white muscle. This protein, when bound to oxygen, imparts a crimson hue to the tissue. It is this aspect as well as an abundant number of mitochondria found in red muscle, compared to white muscle, that impart a much greater aerobic capacity to red muscle (Joanisse, 2004). While T. scripta elegans experiences severe hypoxia over the cold winter months it remains largely inactive in freshwater. It is this combination of low oxygen and inactivity that could initiate atrophy in this aerobic muscle. Phospho-SMAD3 expression was greatly suppressed under anoxia to less than $50 \%$ of the aerobic control value (Figure 2.13) to the control throughout the anoxic episode that gives the first evidence of cell cycle arrest within the tissue's cells. This result is furthered by the matched suppression of c-Myc to $35 \%$ relative expression to control (Figure 2.27), allowing CDK inhibitors to be potentially be expressed and impart their utility in preventing cell cycle progression past the $G_{1}$ phase. Phosphorylation of SMAD2 was also depressed during $20 \mathrm{~h}$ anoxia falling to a relative expression level of $34 \%$ (Figure 2.12). The elevated 5h anoxic expression of phospho-SMAD2 seems to contradict this aspect of the protein's function however, this is discussed in the following section. SMAD4 expression is downregulated as a result of the decreased R-SMAD protein expression during 20h anoxia (Figure 2.14). 


\subsubsection{2 - Angiogenesis Regulation}

As the development of red muscle tissues degrades so does the vasculature to those tissues as they are not required during the winter months and those resources can be diverted to another aspect of the turtle's survival. The initial response to hypoxia is the attempt to increase the blood supply to these tissues as they require oxygen to continue their aerobic metabolic activity. This is supported by the sustained level of SMAD1 and the threefold spike in SMAD5 in 5h anoxia (Figures 2.11 and 2.15). Additionally, total SMAD2 levels begin to increase to 1.5 -fold of the control, and phospho-SMAD2 spikes to a 1.75-fold increased protein expression level (Figure 2.12) suggesting a role in the maintenance of vascular integrity at $5 \mathrm{~h}$ anoxia (Itoh et al., 2012). Once the tissue experiences severely low oxygen availability its vascular structures could join the cells' switch to processes that conserve and protect the limited resources available to the turtle instead of compensating for low energy conditions. SMAD1 relative levels drop to below $40 \%$ of the control in $20 \mathrm{~h}$ anoxia, the first sign of an angiostatic state (Figure 2.11). Total SMAD2 protein expression supports this conjecture with its massive 3.5 -fold increase in its expression effectively stymieing angiogenesis and the maturation of ECs into new vessels. SMAD5 levels continue to be in an elevated state despite no requirement for angiogenesis.

\subsubsection{3 - Oxidative Stress Response}

The general depression in R-SMAD expression as well as the downregulation of the common SMAD partner, SMAD4, has its implications downstream. The red muscle being not well suited for anaerobic function or conditions, will not adapt too well to anoxia. It may still produce molecules of ROS that will not be tolerated well by the cells. The SMAD 
pathway suppression leads to a 1.5 -fold rise in phospho-c-Myc production in $20 \mathrm{~h}$ anoxia (Figure 2.27). This induction can lead to the increased production of $\gamma$-GCS and likely a myriad of other redox enzymes such as SODs and GST.

\section{$\underline{2.4 .4}$ - TGF- $\beta$ Superfamily Mediated Effects in TSE Kidney}

\subsubsection{1 - Cell Cycle Regulation}

In other tissues that experience cell arrest there is a suppression of SMADs 2 and 3 and their phosphorylated analogs, however in kidney there is an added modest complexity. Instead of an immediate and significant drop in protein levels in response to anoxia there was instead an increase in SMAD2 (2.5-fold relative to control) and its phosphorylated form (1.8-fold rise in relative expression) during 5h anoxia (Figure 2.17). As explained in the next section (2.4.4.2 - Angiogenesis) this effect might be due to a requirement for kidney to grow in response to hypoxia in order to prepare to survive weeks without oxygen and circumvent chronic kidney disease which would certainly have fatal consequences (Tanaka and Nangaku, 2013). After this initial response which may seek to compensate for low oxygen availability, kidneys may then shut down the cell cycle as oxygen restriction is prolonged and instead promote the transition to the hypometabolic state. Animal studies have indicated that anoxic damage had been attenuated or even abolished if reabsorptive transport was decreased (Brezis et al., 1984). By 20h of anoxia exposure, phosphorylated SMADs 2 and 3 are reduced (suppressions of $53 \%$ and $26 \%$ of control values, respectively) and could provide indication of reduced cell cycle activity (Figures 2.17 and 2.18). The common SMAD4, in agreement with the R-SMAD patterns observed, is suppressed under both anoxic conditions with a $44 \%$ expression level relative to control at $5 \mathrm{~h}$ anoxia, and a $48 \%$ expression level relative to control at $20 \mathrm{~h}$ anoxia (Figure 2.19). There is a 
concomitant increase in phospho-p21 (1.7-fold rise and 2.9-fold increase during $5 \mathrm{~h}$ and 20h anoxia, respectively), the phosphorylated equivalent of the quintessential CDK inhibitor, during both anoxic durations, despite the total p21 protein levels remaining unchanged over the same periods (Biggar, 2013). Interestingly, in what might be the cell's attempt to reduce the energy expenditure of $\mathrm{CDK}$ inhibitor gene regulation, total p27 protein expression is severely suppressed (reductions to $22 \%$ and $30 \%$ over control protein expression during $5 \mathrm{~h}$ and $20 \mathrm{~h}$ anoxia, respectively) given the heightened differential phosphorylation of its $\mathrm{G}_{1}$ arrest partner, $\mathrm{p} 21$. c-Myc protein expression is intensely upregulated in the red-eared slider kidneys during 20 hours of anoxia to over $210 \%$ of control (Greenway and Storey, 2000). The combination of all these transcription factors may point to the kidney ceasing cell growth by inducing a $\mathrm{G}_{1}$ arrest at 5 h anoxia and a sustainable $\mathrm{G}_{0}$ arrest by $20 \mathrm{~h}$ anoxia and conserving energy in alignment with the tenets of metabolic rate depression (MRD).

\subsubsection{2 - Angiogenesis Regulation}

The kidneys of any organism serve to filter toxicants, waste products, and xenobiotics. The maintenance of this tissue's vascular system (capillaries) is essential for survival and glomerular function, as any disruption in can lead to waste product accumulation (eventually lethal), and disease. Hypoxia plays a role in the dysregulation of angiogenic factors, such as thrombospondin-1, angiostatin and endostatin, which negatively impact capillary formation and regression. More specifically, studies have linked tubulointerstitial hypoxia and hypoxia-inducible transcription factors, such as HIF-1, to the advanced stages of chronic kidney disease in humans (Tanaka and Nangaku, 2013). The turtle is predicted to, therefore, attempt to improve or fully restore the level of connectivity between the 
kidney and the intravascular system of the turtle in order to tolerate anoxic stress throughout its aquatic winter dormancy. Avoiding potentially lethal hypoxic injury to the renal organs while maintaining MRD could be difficult, as renal tubular epithelial cells are among the highest energy consuming cells in the body (Lemons et al., 2010). TGF- $\beta$ cytokine levels increased 1.4-fold during $5 \mathrm{~h}$ anoxia in contrast to the likely suppression of other growth factors associated with angiogenesis (VEGF) during hypoxia (Figure 2.26). SMAD1 was very strongly upregulated in kidney tissues by 2.5 -fold during $5 \mathrm{~h}$ anoxia (Figure 2.16), whereas SMAD5 protein expression was unchanged in response to anoxia (Figure 2.20). SMADs 1 and 5 both stimulate proliferation and migration of endothelial cells while SMADs 2 and 3 inhibit them (Figure 2.33) (Pardali et al., 2010; van Meeteren and ten Dijke, 2012). The results for SMAD 1 could suggest a stimulation of vascularization as an early response to oxygen limitation to the kidneys in an attempt to improve oxygen delivery to the tissue as part of a compensatory response to hypoxia. However, with longer time oxygen levels will fall below a critical level where it is no longer reasonable to try to improve oxygen delivery to tissues and where the conservation responses of metabolic rate depression become more important. Not surprisingly, then, TGF- $\beta$ precursor levels were strongly suppressed to less than $5 \%$ of control expression levels in response to long-term, $20 \mathrm{~h}$ anoxic submergence. This could signal that the kidney switches its status from angiogenic to angiostatic as oxygen availability falls, however the kidney may maintain its vasculature by sustaining the expression level of SMAD1 during $20 \mathrm{~h}$ anoxia at parity with control levels. This theory was also supported by SMAD2 protein levels being increased 1.6-fold relative to control during the $5 \mathrm{~h}$ anoxic exposure (Figure 2.17), providing vascular stability in the endothelium (Itoh et al., 2012). A potential 
secondary level of regulation of these processes are the AP-1 protein complexes that enhance DNA-binding of SMAD complexes in the nucleus to SERPINE1 gene promoters (Figure 2.29). c-Fos experienced a decrease in expression under $20 \mathrm{~h}$ anoxia falling to less than $50 \%$ of its expression in normoxic turtles (Greenway and Storey, 2000). Meanwhile, the whole cell protein expressions of c-Jun (the complex partner of c-Fos) and phospho-cJun were steady, and showed no signs of either upregulation or downregulation, however c-Jun was suppressed during 20h anoxic stress along with p-c-Jun within the nucleus (Biggar, 2013). Using these strategies, the kidneys can be protected from capillary rarefaction, thrombotic microangiopathy, hypertension, and inflammation due to anoxia/anoxic injury (Tanaka and Nangaku, 2013).

\subsubsection{3-Oxidative Stress Response}

In the kidneys the expression pattern of phospho-c-Myc, a protein shown to play a pivotal role in oxidative stress management, mimicked the pattern seen in liver with strong upregulation during both periods of anoxia (1.3-fold increase in 5h anoxia, and 2.9-fold rise in 20h anoxia) (Figure 2.27). Additionally, FoxO proteins are upregulated throughout the duration of the anoxic exposure. FoxO1 and FoxO1 phosphorylated at serine 256 were both upregulated during $5 \mathrm{~h}$ anoxia, while total FoxO3 was upregulated following $20 \mathrm{~h}$ anoxic exposure (Krivoruchko and Storey, 2013). These proteins all work synergistically to prevent a harmful accumulation of ROS within the tissue, and stopping inflammation from occurring. In summary, a defense against ROS and oxidative injury is probably mounted by the kidney through the activation of FoxOs and $\gamma$-GCS enzymatic activity in turtles under anaerobiosis. 


\section{$\underline{2.4 .5 \text { - TGF- } \beta \text { S Superfamily Mediated Effects in TSE Heart }}$}

\subsubsection{1 - Cell Cycle Regulation}

Control of growth and proliferation processes as discussed above is mediated by SMADs 2 and 3 (Goumans et al., 2003; Pardali et al., 2005). Activated phospho-SMAD3 protein concentrations in heart began to descend quickly to $72 \%$ of control aerobic values following $5 \mathrm{~h}$ anoxia, and decreased further to $36 \%$ of control after $20 \mathrm{~h}$ anoxic exposure (Figure 2.23). This should result in the interruption of cardiomyocyte proliferation and growth at 20h anoxia. However, the large 2.2-fold increase in SMAD3 in the cardiac muscle during $5 \mathrm{~h}$ anoxia points to what might be an initial hypertrophic response (Akazawa and Komuro, 2003).

\subsubsection{2 - Angiogenesis Regulation}

Animals with mutations in the Aavrll gene, which encodes the protein ALK-1 (TGF- $\beta$ type I surface receptor), develop a condition called hereditary hemorrhagic telangiectasia (Osler-Weber-Rendu disease) that results in multiple abnormalities in their blood vessels (González-Núñez et al., 2013). One such abnormality includes some arterial vessels flowing directly into veins and completely bypassing the capillaries, known as arteriovenous malformations. Without the blood flow into the capillaries, there is an immense amount of pressure exerted onto the veins (thinner walls and less elastic character compared to arteries) which affects the enlargement of these vessels, and causes severe hemorrhaging. It is this hemorrhaging in organs such as the liver and brain that can be fatal to animals especially those already facing extremely unfavorable environmental factors, such as the red-eared slider and its bouts of anoxia. ALK-1 activates SMADs 1 and 5 which mediate angiogenesis along with secondary roles played by SMADs 2 and 3. SMAD1 
protein expression increased 1.5 -fold in turtle heart during $20 \mathrm{~h}$ anoxia, and remain unchanged during $5 \mathrm{~h}$ anoxic stress (Figure 2.21). In contrast, SMAD5 expression was limited to $32 \%$ of controls. These two SMAD isoforms (SMAD1 and SMAD5) both stimulate angiogenesis so it would seem that these results do not agree with one another, but it appears that SMAD5 wins out given that one of the inhibitors of angiogenesis, SMAD2, is upregulated in response to both $5 \mathrm{~h}$ and $20 \mathrm{~h}$ anoxia (1.7- and 1.5-fold, respectively) in heart (Figure 2.22). Additionally, the relative amount of phosphorylated SMAD 1 and 5 showed overall suppression to 64\% of controls (Figures 2.21 and 2.25) suggesting that the heart is angiostatic in response to anoxia. Levels of the common SMAD4 nearly doubled after $20 \mathrm{~h}$ anoxia in heart proportional to the differential expression of SMAD2 in the oxygen-diminished environment (Figure 2.24). TGF- $\beta$ precursor proteins increased in response to oxygen limitation to turtle heart, but only at $20 \mathrm{~h}$ anoxia when it was upregulated 1.7-fold (Figure 2.26). SMAD1 expression is understood to have a role in protecting the heart from ischemia-reperfusion injury by preventing apoptotic activity in cardiomyocytes during hypoxic and anoxic episodes (Masaki et al., 2005). Furthermore, SMAD1 and SMAD4 through cooperative interaction with GATA4 act as upstream enhancers to the gene $N K X 2-5$, which is responsible for the regulation of cardiac genes and morphogenesis (Brown et al., 2004). The role of the homeobox protein Nkx2-5 to differentiate cardiac progenitor cells and mediate cardioprotection through the elimination of cytotoxic stressors; therefore SMADs 1 and 4 could affect the differentiation of heart cells and play a role in protecting them from ROS (Akazawa and Komuro, 2003). c-Fos, the co-promoter of various proteins, is downregulated in the heart after $20 \mathrm{~h}$ anoxia and provides evidence of the angiostatic nature of the tissue (Greenway and Storey, 2000). 
The theme of an angiogenic hiatus within the heart is a strongly evidenced and may play a critical role as all transcription factors in this organ are either activated or deactivated to potentially promote cell survival rather than growth under low oxygen conditions.

\subsubsection{3 - Oxidative Stress Response}

FoxO1 and FoxO3 protein expressions in response to anoxia have been analyzed in turtle heart tissues. FoxOs play a role in the regulation of cell cycle arrest and oxidative stress resistance in turtles under anaerobiosis. FoxO1 levels were diminished in $5 \mathrm{~h}$ anoxia and the phosphorylated isoform was unchanged throughout the episode, however, the pattern of FoxO3 and p-FoxO3 (Ser253) expressions could indicate the emergence of an antioxidant response beginning at 20h anoxia (Krivoruchko and Storey, 2012). Phosphorylation of FoxO3 at serines 318 and 321 was suppressed at 5h anoxia and remained subdued after $20 \mathrm{~h}$ of anoxic exposure. These data could imply oxidative stress responses in turtle heart are activated by $20 \mathrm{~h}$ of anoxia rather than at $5 \mathrm{~h}$.

ATF4, a downstream transcription factor of the TGF- $\beta$ signaling pathway, binds to the antioxidant response element (ARE) which subsequently promotes the expression of redox enzymes such as GST, SOD, HO-1, and others via its interaction with Nrf2, a "master regulator" of the antioxidant response. Nrf2 is activated by the accumulation of ROS causing its disassociation from Keap1, an Nrf2-binding protein anchored to the cytoskeleton. Its activation can modulate and lead to the expression of hundreds of gene products (Hybertson et al., 2011). ATF4 expression was unchanged during 5h anoxia and upregulated 1.86 -fold after $20 \mathrm{~h}$ anoxia protecting cardiomyocytes from chemical damage caused by anoxia (Krivoruchko and Storey, 2012). Again, this could point to mechanisms 
to prevent oxidative injury being driven forward and expressed and probably initializing at 20h anoxia.

ATF4 proteins typically stimulate the expression of ATF3 proteins which act as coinhibitors to repress the transcription of the IDI gene (Figure 2.29) in combination with nuclear SMAD complexes (Massagué, 2012; Kang et al., 2003). Id proteins (Id1-4) are a subset of transcription factors that contain the highly conserved basic helix-loop-helix (bHLH) motif which is responsible for DNA binding (Wong et al., 2004; Alani et al., 2001). Id proteins lack the basic DNA binding region and typically bind with basic HLH proteins to form heterodimers restricting them from interacting with DNA to affect their signaling abilities; acting as negative regulators of bHLH proteins. Idl activates an antioxidant response to oxidative stress and is known to affect cell cycle progression and proliferation by interacting with and inactivating CDK inhibitors (p16, p21, and p27) (Wong et al., 2004). ATF3 protein levels remained close to control levels after $5 \mathrm{~h}$ in heart, but rose 1.62 -fold in response to $20 \mathrm{~h}$ anoxia repressing the expression of the IDI gene (Krivoruchko and Storey, 2012). It is certainly possible that there is a pre-existing storage of SOD, GST, and peroxidase transcripts, and once the oxygen-deficient episode lasts longer than $5 \mathrm{~h}$, then more enzymes are required to replenish the depleted stock and a stronger antioxidant response could be mounted by the cardiac cells to withstand the accumulation of ROS.

Hypoxia has been previously shown to activate autophagy brought on by HIF-1, a protein closely linked to the SMAD signaling pathway (Glick et al., 2010). Regulation of SMAD transcription factors in the heart cells potentially works to induce cell cycle and mount protective measures against rising ROS concentration due to limited oxygen 
supplied to the cells. Autophagy reduces mitochondrial mass in the affected cells and encourages ATP production in the form of anaerobic glycolysis, rather than oxidative phosphorylation (Glick et al., 2010). This process potentially dampens oxidative stress recognized by the cells and wasteful ATP consumption, accomplishing the goals of metabolic rate depression that is required for the long-term survival of Trachemys scripta elegans throughout its anoxic winter dormancy (Mizushima, 2007). 


\section{$\underline{\text { 2.4.6 - Discussion Figures }}$}

a Synthesis and secretion

1 Pre-pro-TGF $\beta$

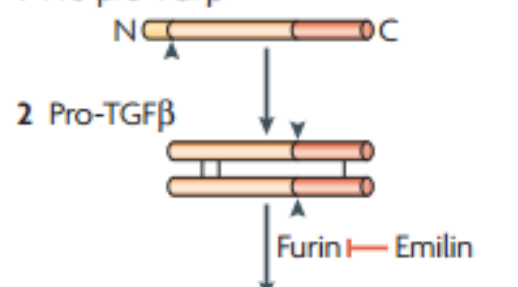

3 SLC
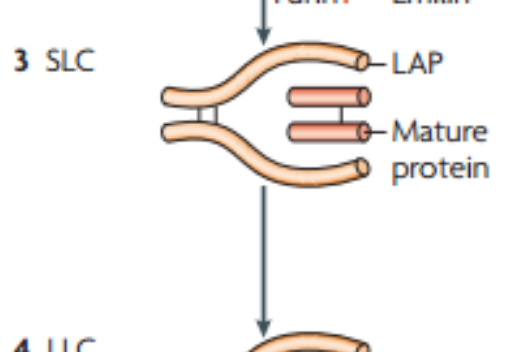

4 LLC
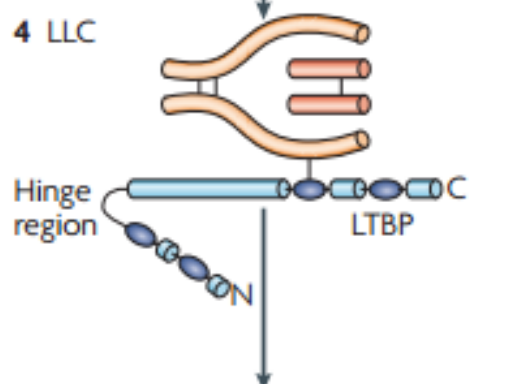

b Activation and receptor binding

5

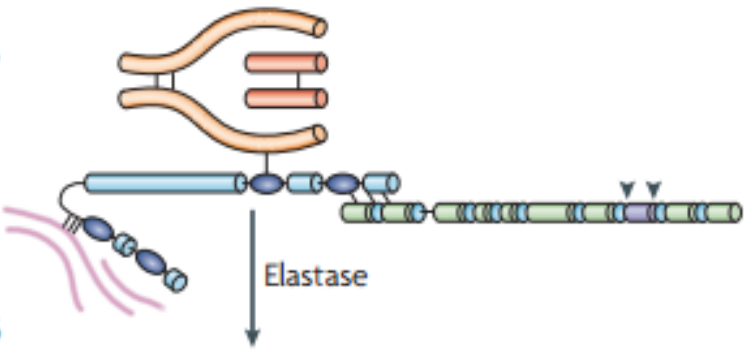

6

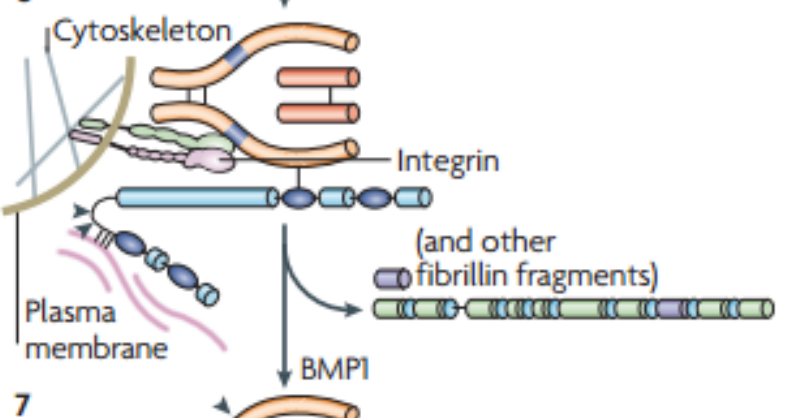

7

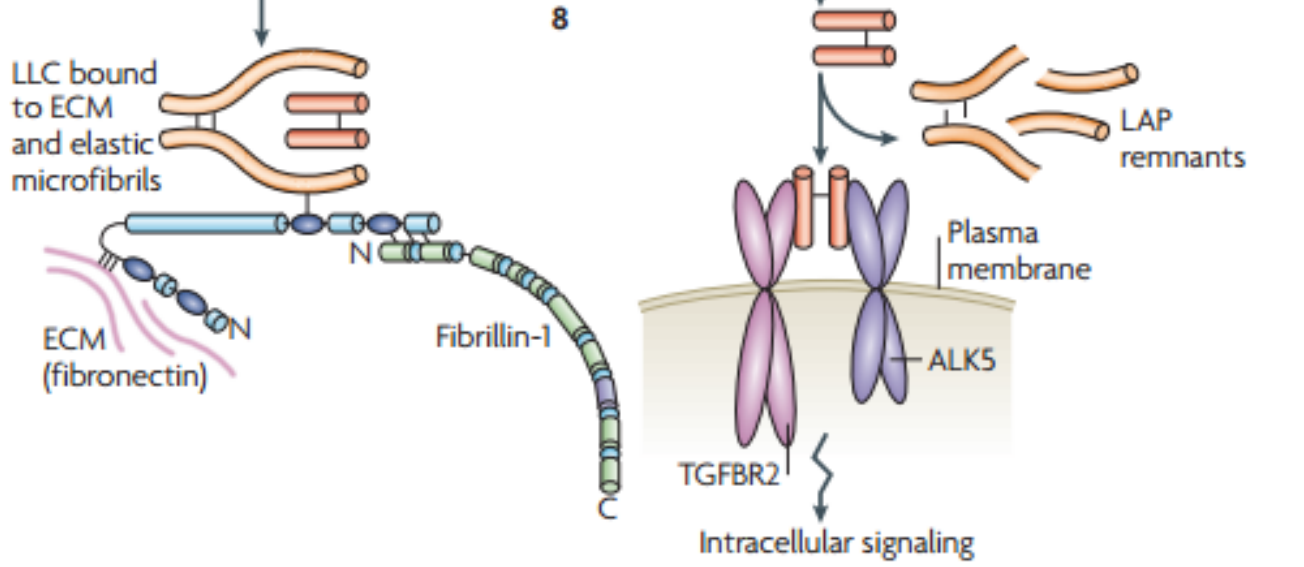

Figure 2.28 - The maturation of TGF- $\beta$ cytokine ligands from precursor form to their activation and receptor binding/signaling. Figure from Ten Dijke and Arthur (2007). 


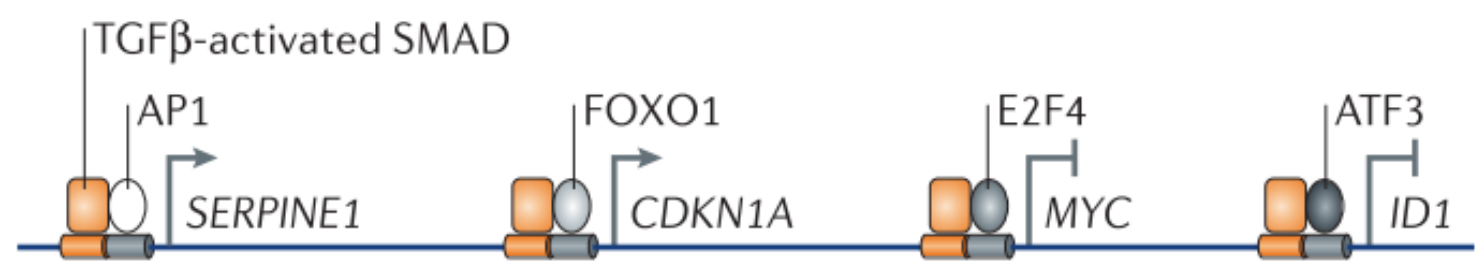

\section{Cellular homeostasis genes}

Figure 2.29 - Transcription factors that regulate homeostasis of a differentiated cell along with their associated genes. Figure reproduced from Massagué (2012).

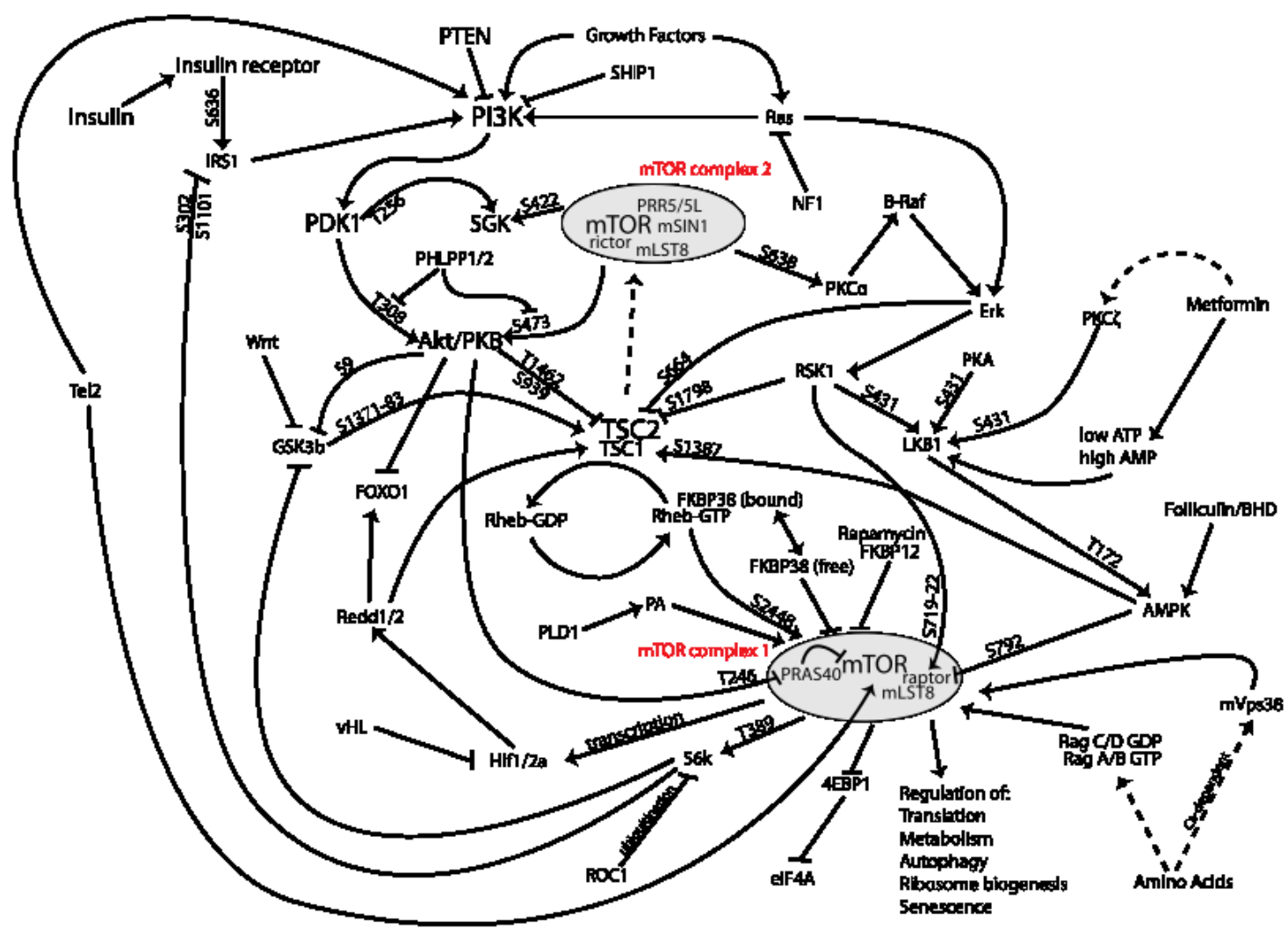

Figure 2.30 - The immense intricacy of the mTOR pathway and its connection to FoxO1, HIF-1, PI3K and Akt. Figure reproduced from Pardali et al. (2010). 

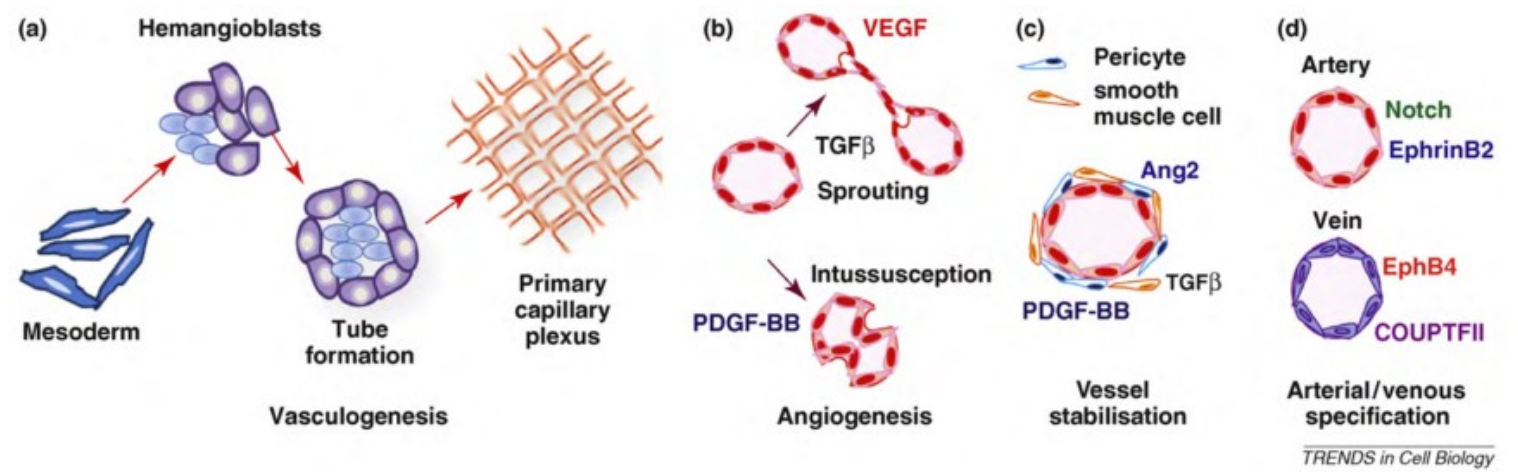

Figure 2.31 - Development of the vascular system. The progressive stages of vessels beginning from vasculogenesis, passing into TGF- $\beta$ or VEGF-mediated angiogenesis and subsequently vessel stabilization through SMCs. Figure reproduced from Pardali et al. (2010).

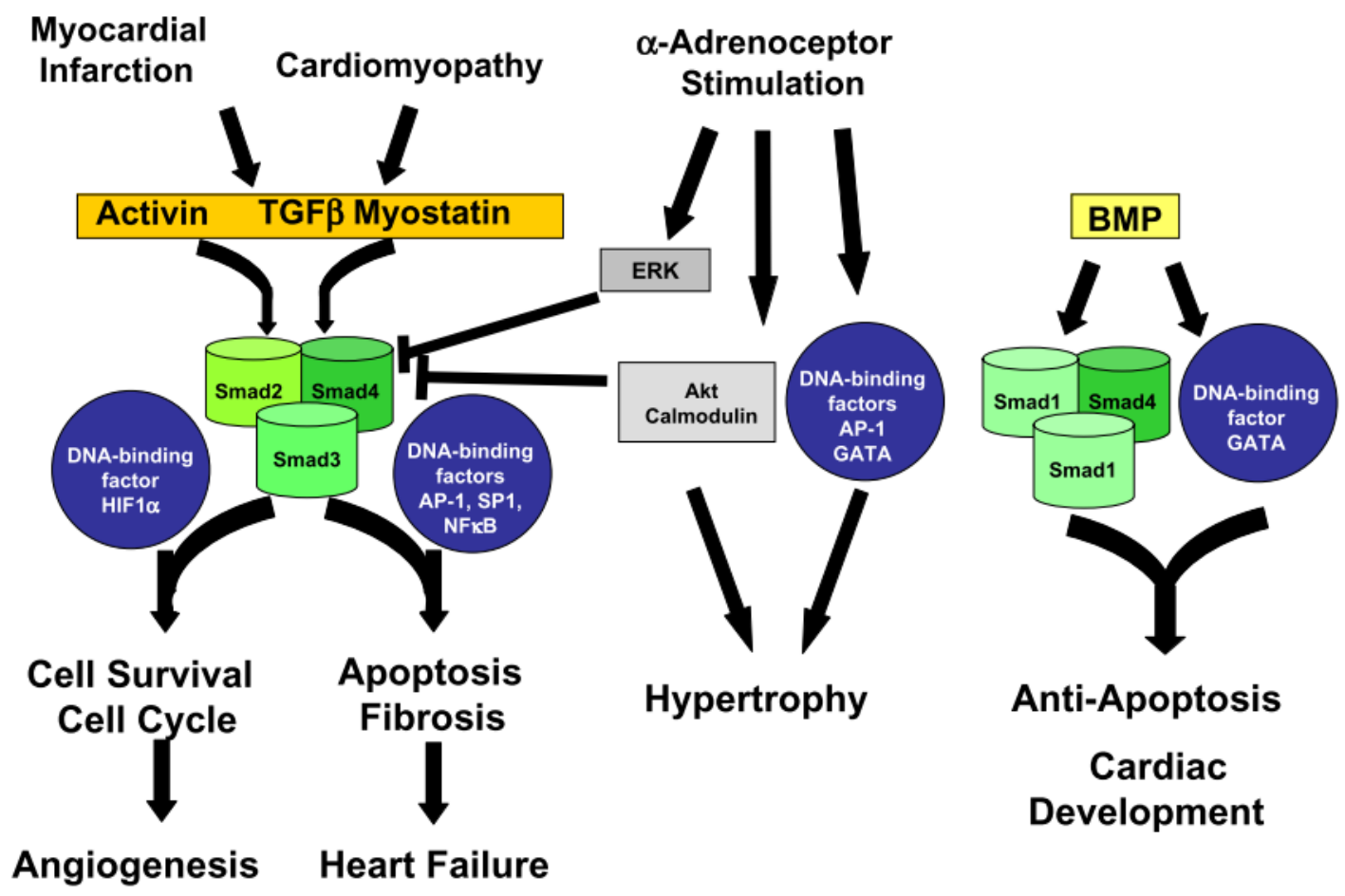

Figure 2.32 - Role of SMADs in angiogenesis, heart failure, and cardiac development. Figure reproduced from Euler-Taimor and Heger (2006). 


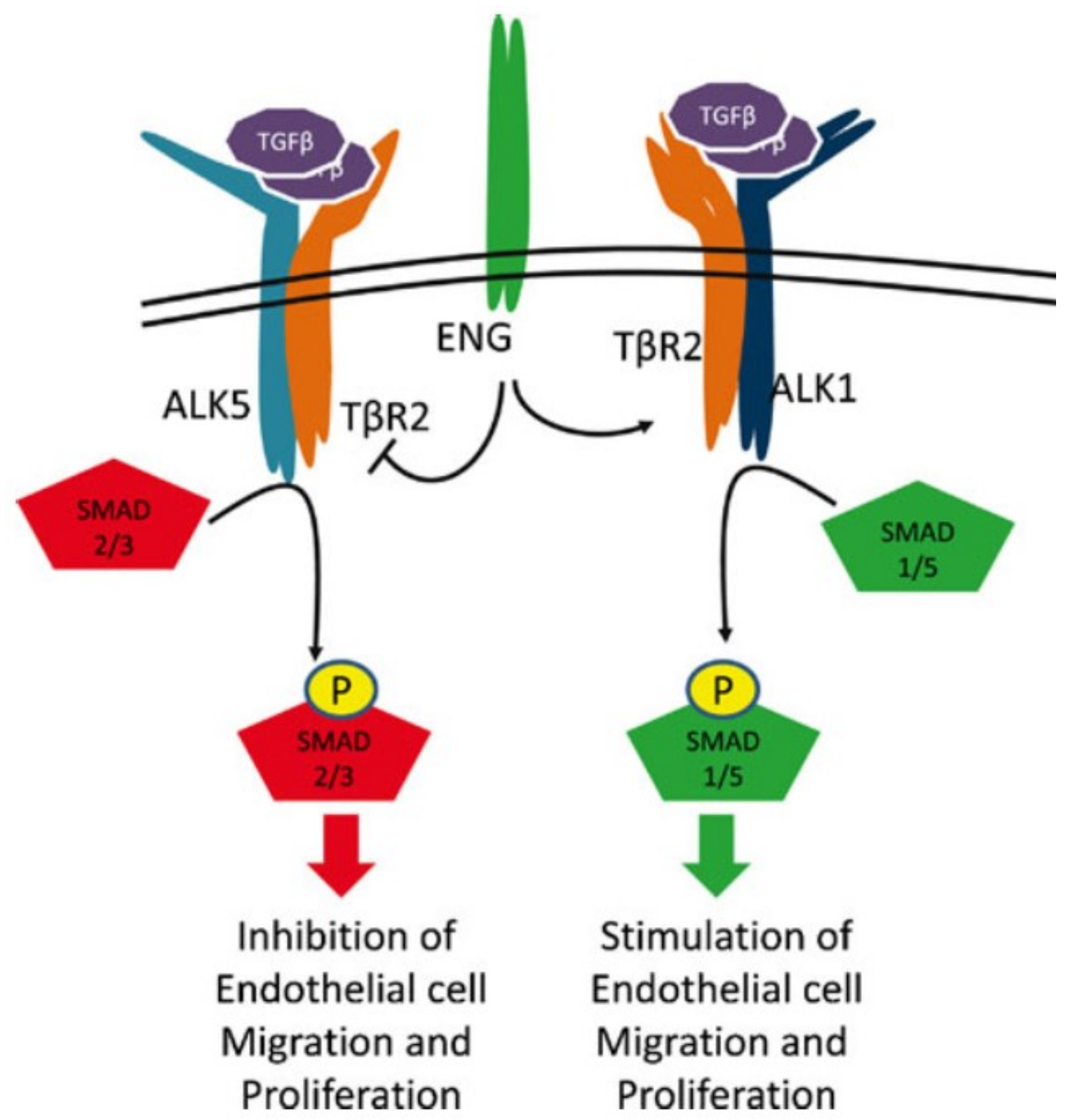

Figure 2.33 - TGF- $\beta$ signaling pathways in endothelial cells. SMADs 2 and 3 inhibit the proliferation and migration of endothelial cells while SMADs 1 and 5 elicit the opposite response and stimulate these two processes. Figure reproduced from van Meeteren and ten Dijke (2012). 


\section{Chapter 3}

\section{Effects of Dehydration Stress on SMAD and its Downstream Protein Expression in Xenopus laevis}

Children waiting for the day they feel good

Happy Birthday, Happy Birthday And I feel the way that every child should 


\section{1 - Introduction}

The African clawed frog, Xenopus laevis, is primarily an aquatic frog with a flat body that has evolved for more ideal swimming. Its hind legs extend sideways and are poor at supporting its body weight on land. The frog derives its name from the Greek words xenos meaning strange, pous meaning foot, and the Latin word laevis, which is an alternate form of lēvis, meaning smooth. The African clawed frog has three-clawed hind legs which it uses to tear apart food, a flattened head and body, and strong, powerful hind legs, common to other members of the Pipidae family, which imparts the frog with its excellent swimming ability. All species of the Pipidae family are nearly completely aquatic and are seldom seen traversing land except in times of extreme drought, when they may move from one body of water to another. Also known as the platanna, X. laevis is native to sub-Saharan Africa (Sudan, Nigeria, and South Africa) (Figure 1.4B), as are all species of the Xenopus genus, and has developed many morphological features to alleviate stresses that arise from the seasonally arid environment - mainly to deal with the stress of dehydration. The frog has been shown to withstand water loss of $30-35 \%$ of its total body water (Malik, 2009; Romspert 1976).

\subsection{1 - Dehydration Stress and Aestivation}

As ponds, rivers, and lakes dry up, X. laevis uses two strategies to try and combat dehydration. The first method is burying into wet mud to reduce the rate of water loss while simultaneously entering aestivation (Storey, 2012). The other method is nocturnal migration to another body of water, but as mentioned before this anuran is not very efficient at travelling over land making this option less favourable (Storey, 2012). In addition to 
these two approaches, the frog also coats itself in a layer of mucous to slow water loss and increases the concentration of electrolytes, nitrogenous osmolytes, and other low molecular weight solutes within its tissues (Hillman, 1978). As the concentration of salts and other solutes increases in its cells, so too does the water potential. The water potential describes a solution's ability to draw in water by way of osmosis; therefore, if the frog increases its cellular water potential that will aid absorption of external water into its body and elevate its chance of survival during the dry season. The main osmolyte synthesized for this function by amphibians is urea (15- to 20-fold increase), along with amino acids and ammonia (twofold to threefold elevation) (Storey, 2012; Balinsky et al., 1967). In an aquatic or damp terrestrial environment, excess nitrogen derived from protein catabolism is typically excreted as ammonia but under dehydrating conditions, the tissues of frogs slow down their excretion of ammonia, and instead up-regulate the enzymes of the urea cycle and synthesize urea. Indeed, carbamoyl phosphate synthase (CPS), the rate determining step of the urea cycle, showed a six-fold increase in the liver of dehydrating frogs (Grundy and Storey, 1994; Balinsky et al., 1961; Janssens, 1964; Seiter et al., 1978). Researchers noted that in studies where Xenopus was left to desiccate naturally for 2-3 months that the amino acid levels in their blood rose by $300 \%$ and blood urea levels increased to 15-20 fold, compared with controls (Balinsky et al., 1961). As an aquatic animal capable of surviving dehydration stress using several evolved coping strategies, $X$. laevis is a key model animal to study signal transduction pathways associated with desiccation tolerance. 


\subsection{2 - Anuran Estivation}

Since X. laevis is native to the sub-Saharan region of Africa, it is exposed annually to high temperatures, arid conditions and low food availability during dry season. Estivation has been described as a state of dormancy, similar to torpor, characterized by inactivity and entry into a hypometabolic state triggered by the frog in response to high temperatures and an arid environment. However, the dormancy is considered to be light as the organism can quickly reverse its state when the stress conditions are removed or when disturbed. In a study done with an estivating snail, Otala lactea, researchers showed that when exposed to wetter conditions (misting with water) the snail was able to awaken from estivation in just ten minutes (Storey, 2002). Estivation is typically a long-term process during which animals spend many months dormant and emerge for only a few weeks of the year for reproduction and nourishment (Storey, 1990). Therefore, estivating animals must have strategies to maintain long-term viability and limit dehydration as much as possible while in the dormant state. In addition to the strategies mentioned earlier (higher urea concentrations, thickening of the surrounding mucous layer), estivating frogs change their breathing to a pattern with prolonged breath-hold (apneic breathing) allowing a reduction in water loss during gas exchange. Most also enter dormancy with large amount of water stored in the bladder, and the water by-production of lipid catabolism also contributes to keeping them hydrated (McClanahan, 1967; DeLaney et al., 1977; Loveridge and Withers, 1981; Barnhart, 1983). Long-term survival means ensuring the amount of fuel reserves in the organism can sustain it for the months that it lays inactive. Part of the equation requires the supply to be large, while the other side of the equation necessitates that the anuran slow down its use of fuel reserves drastically so that they may last long 
periods of time. The method of reducing the energy expenditure of vital processes within the organism is called metabolic rate depression and it plays a major role in the survival of $X$. laevis during long periods of dehydration.

\subsection{3 - Metabolic Rate Depression in Aestivation}

Metabolic rate depression (MRD) occurs in many organisms that must cope with unfavorable environmental conditions. It is a survival phenomenon that is associated with phenomena including torpor, anhydrobiosis, anaerobiosis, diapause, freeze tolerance, and estivation (Storey and Storey, 2010). Desert frogs and toads are known to lower their metabolic rate under dehydration stress to just $10-30 \%$ of their normal resting rate (Storey, 1990). Estivating anurans derive most of their energy from lipid oxidation; e.g. the energy budget of estivating spadefoot toads (Scaphiopus couchii) is fueled by lipids (72\%), protein (23\%) and carbohydrates (5\%). Hypometabolism also requires coordinated suppression of many energy-expensive biochemical and physiological processes to achieve the necessary energy savings, which is why there are strict controls on the physiological and molecular mechanisms involved in oxygen consumption, fuel use, acid-base balance, and patterns of respiration and heartbeat, etc. (Storey, 1990). Regulation at the cellular level involves controls at transcriptional (DNA, transcription factors), post-transcriptional (microRNA, stress granule formation) and translational level (mRNA, protein synthesis) levels to strongly reduce the ATP energy expended on gene expression and protein synthesis (Yamasaki and Anderson, 2008; Wilczynska and Bushell, 2015; Cherkasov et al., 2015). Therefore, the study of transcription factors and their binding to DNA is important to begin to understand how MRD can be used by $X$. laevis to withstand long-term desiccation conditions. 
This study will seek to elucidate the role of SMADs in the dehydrating frog, and furthermore, study its effects on transcription factors downstream. In Chapter 1, I surmised that Xenopus laevis would seek to lower all of its R-SMADs in hopes of achieving cell cycle arrest and may even play a role in the antioxidant response since dehydration can lead to ROS generation.

\section{2 - Methods and Materials}

\subsection{1 - Animal Treatments}

Adult African clawed frogs (X. laevis) were obtained from breeding colonies at the Department of Zoology (University of Toronto). Prior to experimentation, frogs were held at $22 \pm 1{ }^{\circ} \mathrm{C}$ in tanks with dechlorinated water for 1-2 weeks. Frogs were fed three times per week (CU Adult Frog diet, PMI Nutrition International) and water was changed on the day after each feeding. Frogs were then divided into control and dehydrated groups and were not fed again during the experimental period. Controls (CXL) were maintained in the same conditions as above. For dehydration, frogs were removed from water, quickly weighed and placed into dry containers at $22^{\circ} \mathrm{C}$. Animals were allowed to lose water over time, as determined by body mass measurements at various time intervals until the target level of water loss was reached. The percentage of total body water lost was calculated as follows:

$$
\% \Delta_{H_{2} O}=\left[\frac{m_{i}-m_{d}}{m_{i}-B W C_{i}}\right] \times 100 \%
$$

where $\mathrm{m}_{\mathrm{i}}, \mathrm{m}_{\mathrm{d}}$, and $\mathrm{BWC}_{\mathrm{i}}$ are initial mass, dehydrated mass, and initial body water content of X. laevis frogs, respectively. Mean initial body water content was previously determined to be 
$(0.74 \pm 0.02) \mathrm{g} \mathrm{H}_{2} \mathrm{O} \mathrm{g}^{-1}$ body mass (Malik and Storey, 2009). Frogs were sampled when mean total body water loss was about $15-16 \%$ or $30-32 \%$; for experiments already completed mean values were $16.4 \pm 0.3 \%$ for medium dehydration (MD) and $31.2 \pm 0.8 \%$ for high dehydration (HD). All frogs were killed by pithing and then tissue samples were quickly dissected out, immediately frozen in liquid nitrogen and transferred to $-80^{\circ} \mathrm{C}$ until use. All animals were cared for in accordance with the guidelines of the Canadian Council on Animal Care, and all experimental procedures had the prior approval of the Carleton University Animal Care Committee.

\subsection{2 - Homogenization of Animal Tissues and Total Protein Isolation}

Total protein was extracted from liver and skeletal muscle samples from control, medium and high dehydration treated frogs. Frozen tissues $(\sim 0.5 \mathrm{~g})$ were crushed under liquid nitrogen and homogenized in $1 \mathrm{~mL}$ of homogenization buffer [20 mM 4-(2hydroxyethyl)-1-piperazineethanesulfonic acid (HEPES) pH 7.5, $200 \mathrm{mM} \mathrm{NaCl}, 0.1 \mathrm{mM}$ ethylenediamine tetraacetate (EDTA), $10 \mathrm{mM}$ NaF, $1 \mathrm{mM}$ sodium orthovanadate $\left(\mathrm{Na}_{3} \mathrm{VO}_{4}\right), 10 \mathrm{mM} \beta$-glycerophosphate (BGP)] with a few crystals of phenylmethylsulfonyl fluoride (PMSF) and $1 \mu \mathrm{L}$ of protease inhibitor cocktail [104 mM 4-(2-aminoethyl)benzenesulfonylfluoride hydrochloride (AEBSF), $80 \mu \mathrm{M}$ aprotinin, $4 \mathrm{mM}$ bestatin, $1.4 \mathrm{mM}$ E-64, 2 mM leupeptin, 1.5 mM pepstatin A; Sigma-Aldrich, Oakville, ON, Canada] added immediately before use. After homogenization the samples were centrifuged at $10,000 \times g$ for 15 minutes at $4^{\circ} \mathrm{C}$ and the supernatant was collected.

Soluble protein concentration was determined using the Bio-Rad Protein Assay (5000006; Bio-Rad Laboratories (Canada) Ltd., Mississauga, ON, Canada) using bovine serum albumen (BSA) as the standard. The assay was carried out as follows: one volume of stock 
protein assay concentrate was diluted with four volumes of $\mathrm{ddH}_{2} \mathrm{O}$ to make a working BioRad protein reagent. Into each non-binding microplate well, $10 \mu \mathrm{L}$ of the protein sample was added along with $190 \mu \mathrm{L}$ of the working Bio-Rad reagent. This assay was done in triplicate to ensure accurate protein concentrations. The well contents were allowed to react for five minutes, before the microplate was inserted into a BioTek Powerwave HT (Cat \# RPRWI; Norgen Biotek Corp., Thorold, ON) and read using the associated BioTek Gen5 ${ }^{\mathrm{TM}}$ data analysis software at a wavelength of $595 \mathrm{~nm}$.

Sample concentrations were then adjusted to $10 \mu \mathrm{g} / \mu \mathrm{L}$ by addition of a small volumes of homogenization buffer. Samples were then mixed 1:1 v:v with 2x SDS loading buffer [100 mM Tris(hydroxymethyl)aminomethane (Tris) base, 4\% w/v sodium dodecyl sulphate (SDS), 20\% v/v glycerol, $0.2 \%$ w/v bromophenol blue, $10 \%$ v/v 2mercaptoethanol $(\mathrm{MeSH})]$ for a final protein concentration of $5 \mu \mathrm{g} / \mu \mathrm{L}$. Samples were boiled for five minutes to denature the proteins, immediately cooled on ice and stored at $-40^{\circ} \mathrm{C}$ until use.

\subsection{3 - SDS Polyacrylamide Gel Electrophoresis and Immunoblotting}

Discontinuous, 15-well SDS-polyacrylamide gels [10\% v/v acrylamide/bis-acrylamide, $400 \mathrm{mM}$ Tris base $\mathrm{pH} 8.8,0.1 \% \mathrm{w} / \mathrm{v}$ SDS, $0.1 \% \mathrm{w} / \mathrm{v}$ ammonium persulfate (APS), $0.04 \%$ v/v N,N,N',N'-tetramethylethylenediamine (TEMED)] of a $1.00 \mathrm{~mm}$ thickness, were prepared with 5\% upper, stacking gels $(5 \% \mathrm{v} / \mathrm{v}$ acrylamide, $130 \mathrm{mM}$ Tris base $\mathrm{pH} 6.8$, $0.1 \% \mathrm{w} / \mathrm{v}$ SDS, $0.1 \% \mathrm{w} / \mathrm{v}$ APS, $0.1 \% \mathrm{v} / \mathrm{v}$ TEMED). For each gel, an equal amount of soluble protein $(20-25 \mu \mathrm{g})$ was loaded into each well and proteins were separated electrophoretically in SDS-PAGE running buffer (25 mM Tris base, $190 \mathrm{mM}$ glycine, $0.1 \%$ w/v SDS) at $180 \mathrm{~V}$ for 50-60 minutes using either a Bio-Rad Mini Protean III system or a 
Bio-Rad Mini Protean Tetra Cell system (Bio-Rad Laboratories, Mississauga, ON). In order to estimate the size of the proteins as well as the efficiency of the wet transfer, a 3-5 $\mu \mathrm{L}$ aliquot of PiNK plus pre-stained molecular mass ladder (PM005-0500; FroggaBio, Toronto, ON) was loaded in one well. After electrophoresis, proteins were electroblotted onto a 0.45 micron pore size polyvinylidene difluoride (PVDF) membrane (Immobilon-P membrane, IPVH00010; Millipore, Etobicoke, ON) by wet transfer with a transfer buffer solution ( $25 \mathrm{mM}$ Tris base $\mathrm{pH} 8.5,192 \mathrm{mM}$ glycine, and $20 \% \mathrm{v} / \mathrm{v}$ methanol) at $4{ }^{\circ} \mathrm{C}$ for 90 minutes at $160 \mathrm{~mA}$. If a protein was less than $20 \mathrm{kDa}$, the wet transfer parameters were switched to $30 \mathrm{~V}$ for 45 minutes at $4^{\circ} \mathrm{C}$. The membrane was washed three times for 5 minutes each with 3-5 mL of Tris-buffered saline containing Tween-20 (TBST: $20 \mathrm{mM}$ Tris base, $140 \mathrm{mM}$ sodium chloride $(\mathrm{NaCl}), 0.05 \% \mathrm{v} / \mathrm{v}$ Tween-20). The blots were then blocked with either low molecular weight polyvinyl alcohol (PVA) (30-70 kDa: made at 1 $\mathrm{mg} / \mathrm{mL}$ in TBST, and boiled for 10 minutes) for 45 seconds, or with $10 \%$ milk for 30 minutes (for small proteins), followed by three 5-minute washes using TBST. Blots were then incubated overnight at $4^{\circ} \mathrm{C}$ with primary antibody diluted in TBST $(1: 1000 \mathrm{v} / \mathrm{v})$ with a small amount of sodium azide added; the blocking and antibody dilutions differed for each antibody (details of each western blotting condition provided in table in APPENDIX B). Membranes were then washed multiple times with TBST and distilled/deionized water, and incubated with appropriate secondary antibody conjugated with horseradish peroxidase (HRP; Bioshop Canada, Burlington, ON, Canada) 1:8000 v/v in TBST for 45 minutes. Membranes were again washed with TBST and signal was detected using enhanced chemiluminescence (ECL); $1.2 \mathrm{ml}$ of $1: 1 \mathrm{v} / \mathrm{v}$ substrate solutions (hydrogen peroxide and luminol reagent) were first thoroughly mixed and then added to the membrane 
to initiate exposure. The resulting chemiluminescence signal was detected using either a Chemi-Genius bioimaging system or a G:BOX bioimaging system (Syngene Co., Frederick, MD, USA), combined with the Syngene GeneSnap software and band densities were analyzed using the associated GeneTools software. Membranes were then stained for 10 minutes with Coomassie blue $[0.25 \% \mathrm{w} / \mathrm{v}$ Coomassie Brilliant Blue $\mathrm{R}$ in $50 \% \mathrm{v} / \mathrm{v}$ methanol $(\mathrm{MeOH}), 7.5 \% \mathrm{v} / \mathrm{v}$ acetic acid $\left.\left(\mathrm{CH}_{3} \mathrm{COOH}\right)\right]$ and destained for $\sim 15 \mathrm{~min}$ with destaining mix $\left[60 \% \mathrm{v} / \mathrm{v} \mathrm{MeOH}, 20 \% \mathrm{v} / \mathrm{v} \mathrm{CH}_{3} \mathrm{COOH}\right.$ in deionized water $\left.\left(\mathrm{ddH}_{2} \mathrm{O}\right)\right]$.

\section{$\underline{3.2 .4-\text { Statistical Analysis }}$}

Detection of bands on gels and blots used the ChemiGenius Bio-Imaging System (Syngene) and densitometric analysis was performed with the associated Gene Tools software. Immunoblot band intensity in each lane was normalized against a set of strong Coomassie blue-stained bands in the same lane to correct any minor variations in sample loading; the Coomassie stained band chosen showed constant intensity across all samples and was well-separated from the area of the gel containing the immunoreactive protein. Mean normalized band densities \pm standard error of the mean (SEM) were calculated and significance testing used one-way analysis of variance (ANOVA) followed by Tukey's post-hoc testing with a significance level threshold of $p=0.05$. All data are derived from multiple independent tissue extracts from different animals $(n=3-5)$. Tukey's range test statistic is calculated using the following equation:

$$
q_{S}=\frac{Y_{A}-Y_{B}}{S E}=\frac{\left(\bar{y}_{\max }-\bar{y}_{\min }\right)}{S \sqrt{\frac{2}{n}}}
$$


Where $\mathrm{q}_{\mathrm{s}}$ is the studentised range statistic, $\mathrm{SE}$ is the standard error of the data, $\mathrm{S}^{2}$ is the pooled variance of the samples, $\mathrm{y}_{\max }\left(\mathrm{Y}_{\mathrm{A}}\right)$ is the larger mean being tested, and $\mathrm{y}_{\min }\left(\mathrm{Y}_{\mathrm{B}}\right)$ is the smaller mean being tested.

\section{3 - Results}

The antibodies used in this study cross-reacted with only a single protein band at the expected molecular masses of the target proteins after electrophoresis of $X$. laevis tissue extracts. The proteins analyzed were as follows with their bioinformatically identified molecular weight in brackets using the National Center for Biotechnology Information (NCBI: http://www.ncbi.nlm.nih.gov): SMAD1 (52 kDa), SMAD2 (52 kDa), SMAD3 (48 kDa), SMAD4 (60 kDa), SMAD5 (52 kDa), c-Myc (47 kDa), c-Fos (40 kDa), and TGF- $\beta$ precursor (44 kDa). Specific $X$. laevis protein sequences can be found in APPENDIX A.

\subsection{1 - Effects of Dehydration on SMAD Expression in Liver}

In the liver, SMAD1 increased significantly under medium dehydration, but the increase was not significant in the high dehydration condition, compared to control (MD: $1.312 \pm$ 0.092, $p<0.05 ;$ HD: $1.251 \pm 0.026$, N.S.) (Figure 3.1). SMAD5 levels did not change across the two desiccation conditions, medium (MD: $0.937 \pm 0.061$, N.S.) and high (HD: $1.028 \pm$ 0.180$, N.S.), compared to the frogs that were kept hydrated (CXL: $1.000 \pm 0.085)$ (Figure 3.5). Levels of phosphorylated SMAD1 and SMAD5 showed a gradual decrease with dehydration from the non-significant $92 \%$ of control levels under medium dehydration to $82 \%$ of control expression under high dehydration stress (MD: $0.921 \pm 0.032$, N.S.; HD: $0.818 \pm 0.024, p<0.05)$ 
SMAD2 levels did not change significantly under either medium or high dehydration compared to hydrated frogs (MD: $0.873 \pm 0.065$, N.S.; HD: $0.840 \pm 0.091$, N.S.) (Figure 3.2). The activated form, phospho-SMAD2, also showed no change in differential phosphorylation when considering medium (MD: $0.877 \pm 0.052$, N.S.) and high dehydration (HD: $1.020 \pm 0.041$, N.S.) frogs compared to control frogs (CXL: $1.000 \pm$ 0.032). SMAD3 content dropped to $76.7 \%$ and subsequently to $70.1 \%$ of control levels during medium and high dehydration, respectively (MD: $0.767 \pm 0.025, p<0.01$; HD: 0.704 $\pm 0.042, p<0.01$ ) (Figure 3.3). However, phosphorylation of the protein (p-SMAD3) did not change significantly under dehydration (MD: $0.955 \pm 0.080$, N.S.; HD: $0.793 \pm 0.061$, N.S.), compared to controls (CXL: $1.000 \pm 0.037)$. Levels of co-SMAD4 did not change significantly under either medium or high desiccation stress, as compared with controls (MD: $0.924 \pm 0.60$, N.S.; HD: $1.186 \pm 0.070$, N.S.) (Figure 3.4). However, the values for MD and HD groups were significantly different, varying by $26 \%(p<0.05)$. 


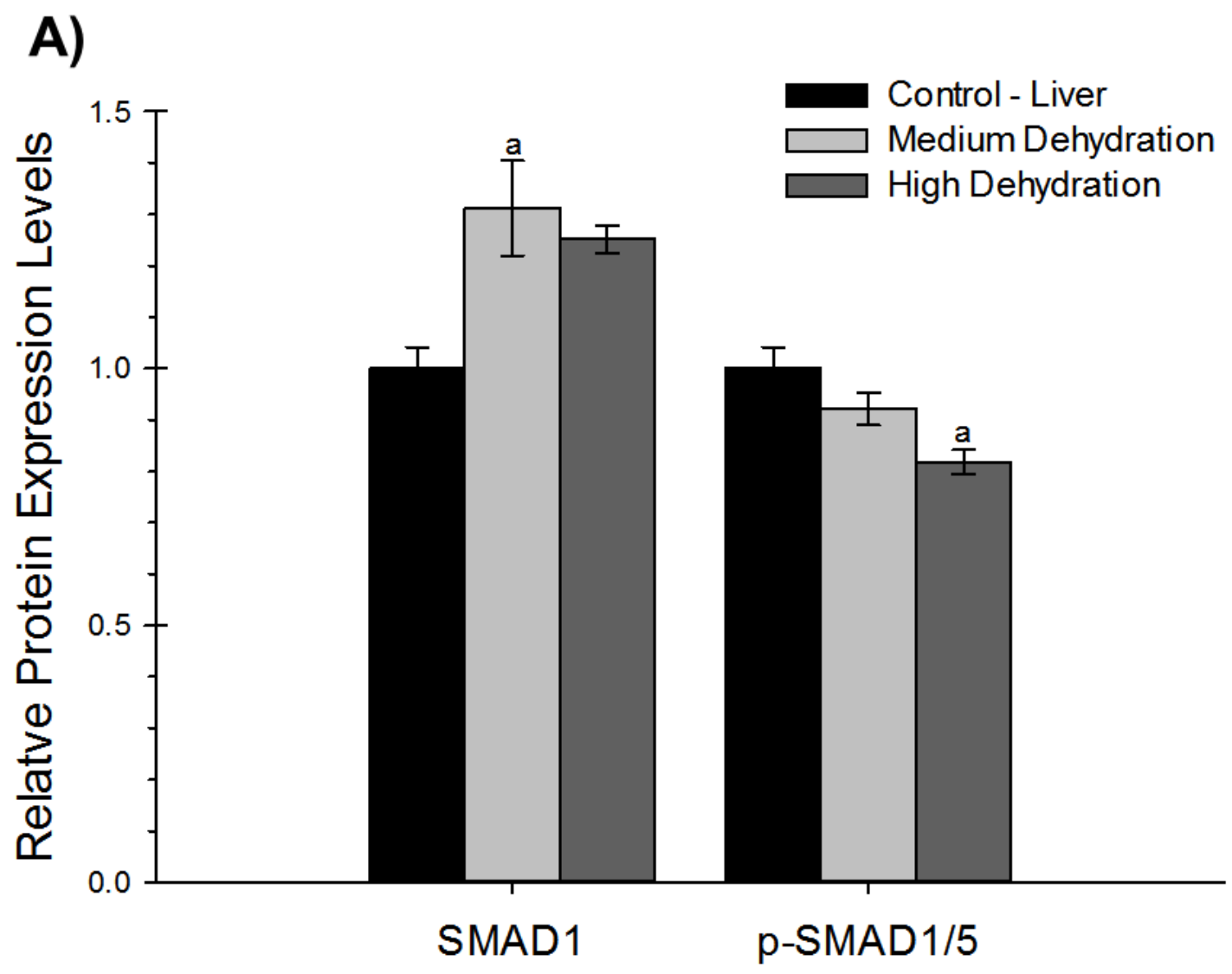

B)

\section{SMAD1}

\section{p-SMAD1/5}

Figure 3.1 - Effects of medium and high dehydration on the protein expression of SMAD1 (52 kDa) and phospho-SMAD1 (serine 463/465) in African clawed frog liver as determined by immunoblotting. A histogram of normalized mean protein levels (A) is shown along with representative immunoblots (B). Data are means \pm S.E.M., $n=3-4$ independent trials. Significantly different from the corresponding $(a)$ control or $(b)$ medium dehydration values using a one-way ANOVA and a Tukey's post-hoc test, $p<0.05$. 


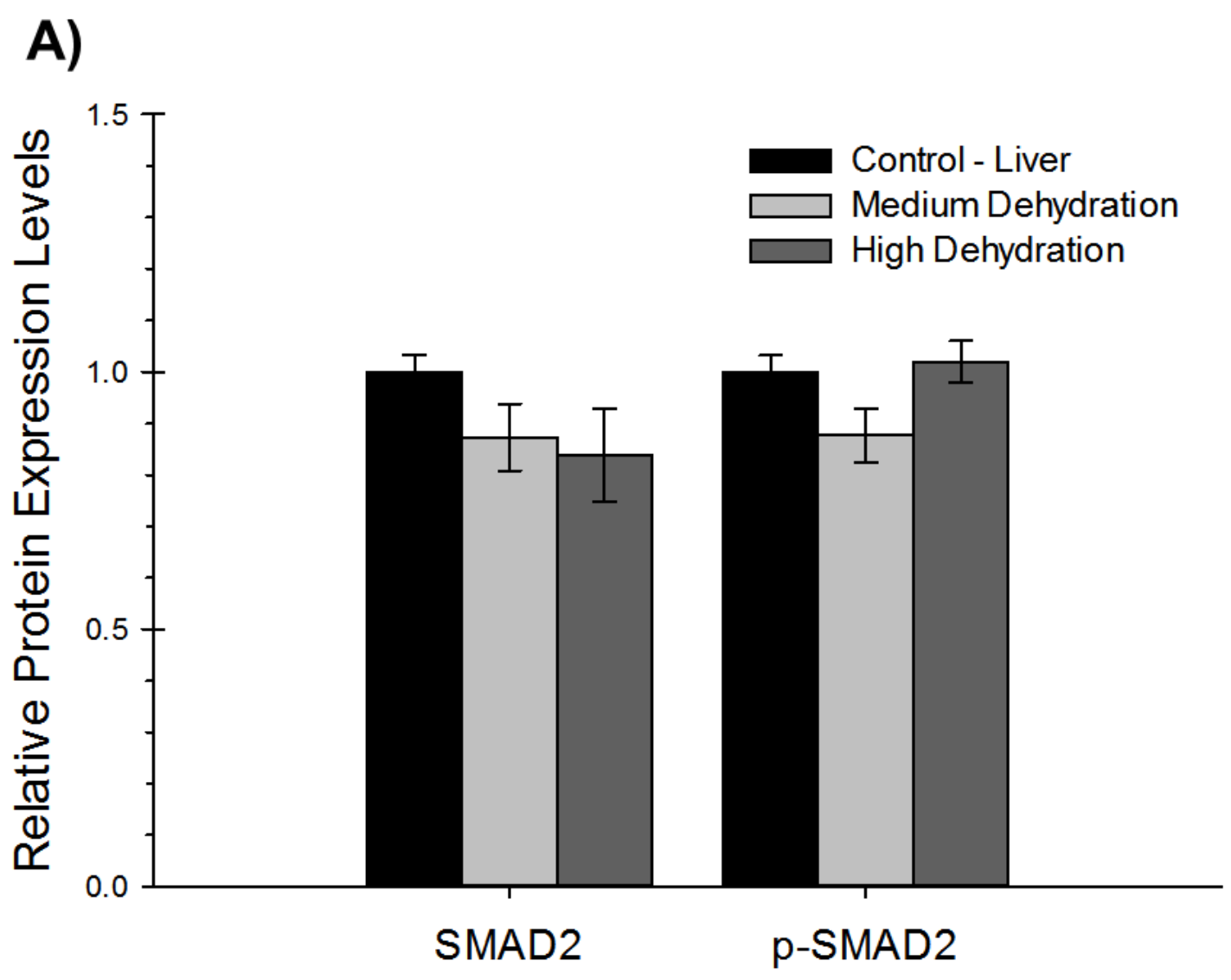

B)

\section{SMAD2}

p-SMAD2

Figure 3.2 - Effects of medium and high dehydration on the protein expression of SMAD2 (52 kDa) and phospho-SMAD2 (serine 465/467) in African clawed frog liver as determined by immunoblotting. A histogram of normalized mean protein levels (A) is shown along with representative immunoblots $(\mathbf{B})$. Data are means \pm S.E.M., $n=3-4$ independent trials. Significantly different from the corresponding $(a)$ control or $(b)$ medium dehydration values using a one-way ANOVA and a Tukey's post-hoc test, $p<0.05$. 

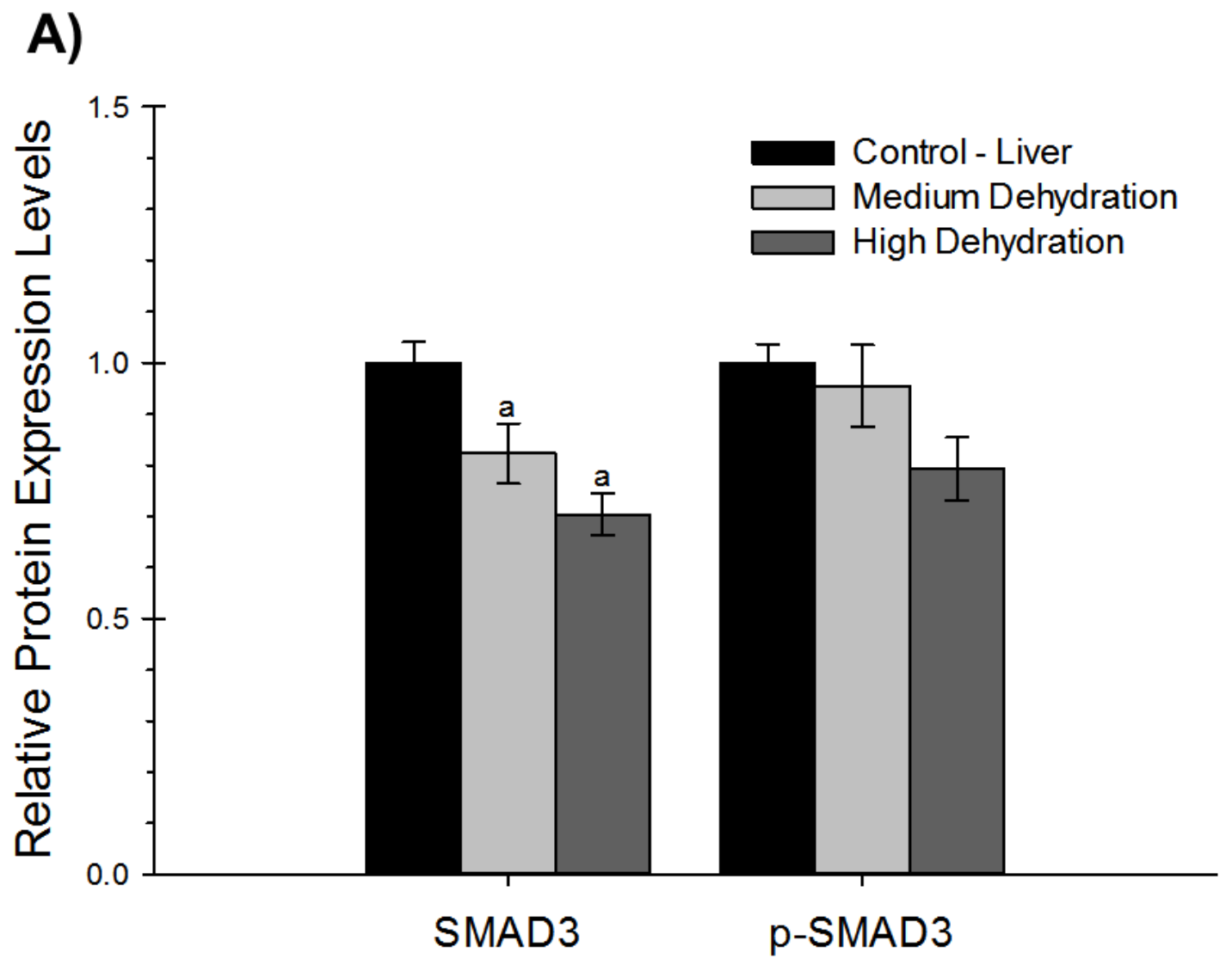

B)

\section{SMAD3}
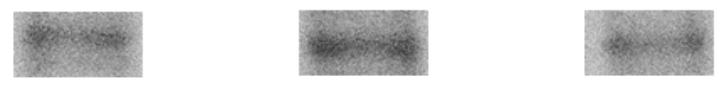

p-SMAD3
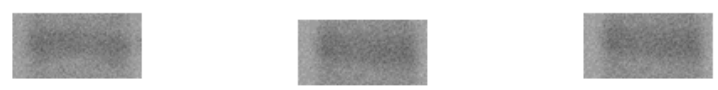

Figure 3.3 - Effects of medium and high dehydration on the protein expression of SMAD3 (48 kDa) and phospho-SMAD3 (serine 423/425) in African clawed frog liver as determined by immunoblotting. A histogram of normalized mean protein levels (A) is shown along with representative immunoblots (B). Data are means \pm S.E.M., $n=3-4$ independent trials. Significantly different from the corresponding ( $a$ ) control or $(b)$ medium dehydration values using a one-way ANOVA and a Tukey's post-hoc test, $p<0.05$. 


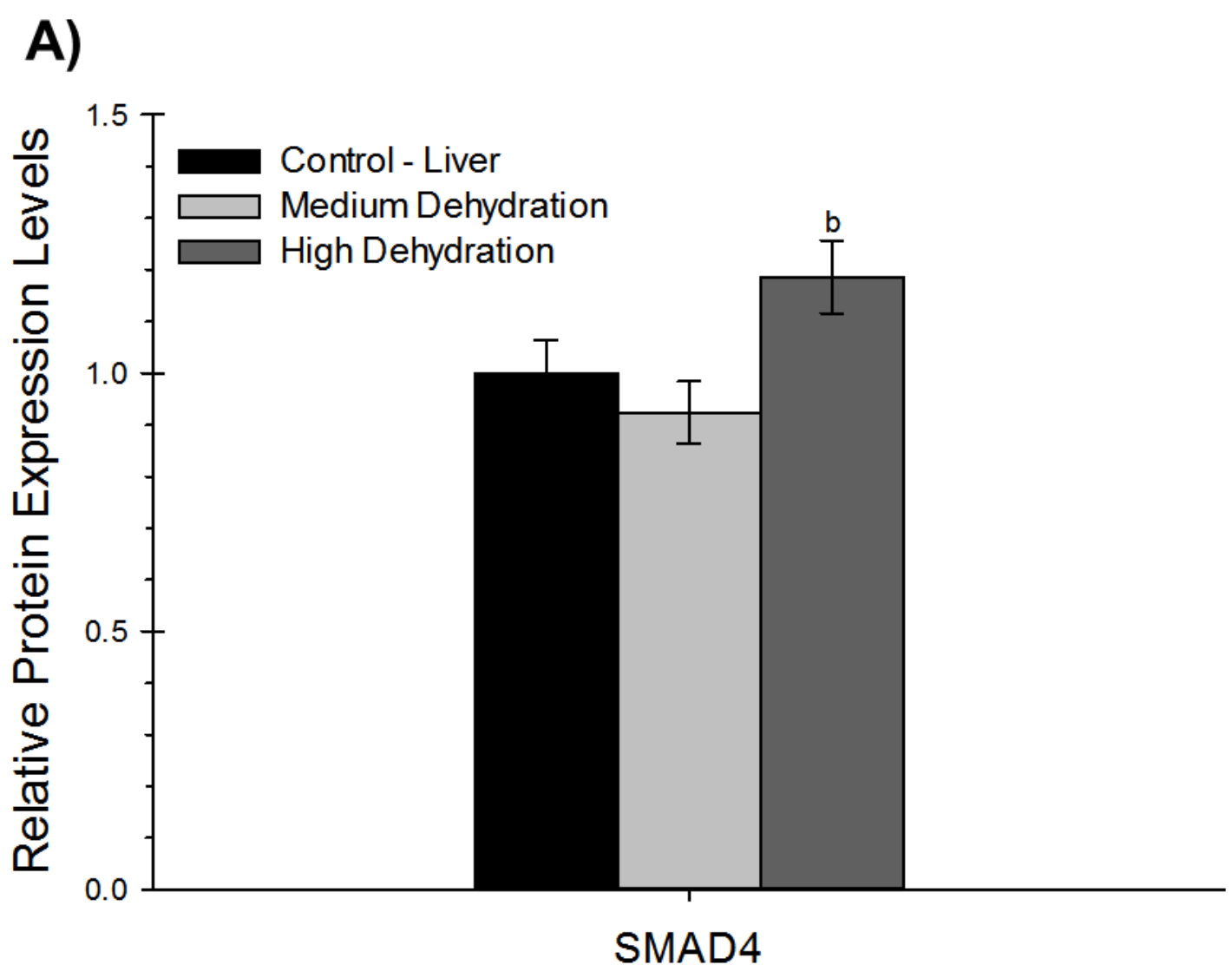

B)

\section{SMAD4}

Figure 3.4 - Effects of medium and high dehydration on the protein expression of SMAD4 (60 kDa) in African clawed frog liver as determined by immunoblotting. A histogram of normalized mean protein levels $(\mathbf{A})$ is shown along with representative immunoblots (B). Data are means \pm S.E.M., $n=3-4$ independent trials. Significantly different from the corresponding $(a)$ control or $(b)$ medium dehydration values using a one-way ANOVA and a Tukey's post-hoc test, $p<0.05$. 


\section{A)}

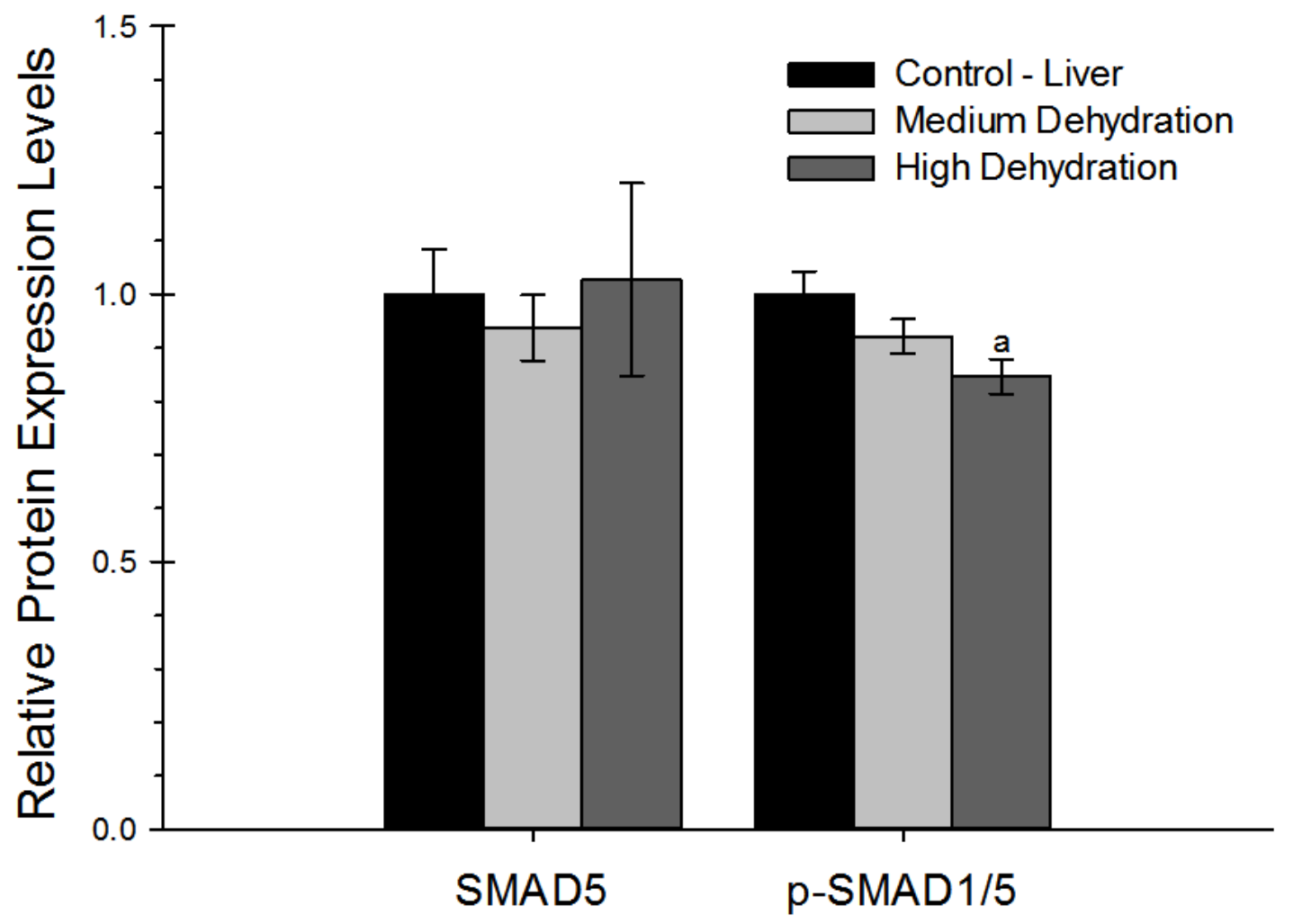

B)

\section{SMAD5}

\section{p-SMAD1/5}

Figure 3.5 - Effects of medium and high dehydration on the protein expression of SMAD5 (52 kDa) and phospho-SMAD1/5 (serine 463/465) in African clawed frog liver as determined by immunoblotting. A histogram of normalized mean protein levels (A) is shown along with representative immunoblots (B). Data are means \pm S.E.M., $n=3-4$ independent trials. Significantly different from the corresponding $(a)$ control or (b) medium dehydration values using a one-way ANOVA and a Tukey's post-hoc test, $p<0.05$. 


\subsection{2 - Effects of Dehydration on SMAD Expression in Muscle}

In frog muscle, large changes observed when it came to the effects of dehydration on SMAD1 protein expression. SMAD1 levels decreased significantly to $73 \%$ of controls under medium dehydration (MD: $0.730 \pm 0.071, p<0.05$ ), while the drop from control to high dehydration was more even greater at $57 \%$ of the expression levels of the control (HD: $0.567 \pm 0.060, p<0.005)$ (Figure 3.6). However, SMAD5 total protein expression showed an increased trend (1.25-fold and 1.30-fold compared to controls) but values for medium and high dehydration conditions were not significant with the post-hoc Tukey test (MD: $1.254 \pm 0.058$, N.S.; HD: $1.295 \pm 0.091$, N.S.) (Figure 3.10). The combined phosphorylation levels of these two proteins, detected by a p-SMAD1/5 antibody, decreased strongly under medium dehydration stress to $60 \%$ of control values but rebounded to near control values in the high dehydration condition $(89.4 \%$ of the control value) (MD: $0.598 \pm 0.030, p<0.01$; HD: $0.894 \pm 0.047, N . S$.). Values for $\mathrm{p}-\mathrm{SMAD1} / 5$ expression in MD and HD conditions were significantly different $(p<0.01)$.

SMAD2 expression was observed to be $73 \%$ at medium dehydration, and protein expression significantly decreased to $47 \%$ in high dehydration stress (MD: $0.727 \pm 0.094$, N.S.; HD: $0.472 \pm 0.061, p<0.005$ ) (Figure 3.7). Levels of p-SMAD2, the activated form of SMAD2, showed no significant changes in its phosphorylation state during both desiccation conditions (MD: $0.764 \pm 0.067$, N.S.; HD: $1.245 \pm 0.063$, N.S.). Although both dehydrated groups were not significantly different from the control group, MD and HD values differed significantly $(p<0.01)$. SMAD3 protein expression dropped significantly under both stress conditions compared to controls (MD: $0.822 \pm 0.026, p<0.05$; HD: 0.753 \pm 0.023, $p<0.005$ ) (Figure 3.8). Phosphorylated SMAD3, meanwhile, decreased 
significantly only when under high desiccation stress (HD: $0.831 \pm 0.014, p<0.05$ ) compared to controls (CXL: $1.000 \pm 0.044$ ), and was also significant lower than the MD value $(p<0.001 ; \mathrm{MD}: 1.108 \pm 0.020$, N.S. vs CXL). The co-SMAD, SMAD4, showed no changes in protein levels under both dehydration stress conditions (medium and high) displaying expression levels of $78 \%$ and $80 \%$, respectively (MD: $0.775 \pm 0.045$, N.S.; HD: $0.796 \pm 0.076$, N.S.), and the values were not significantly different from controls (Figure 3.9). 


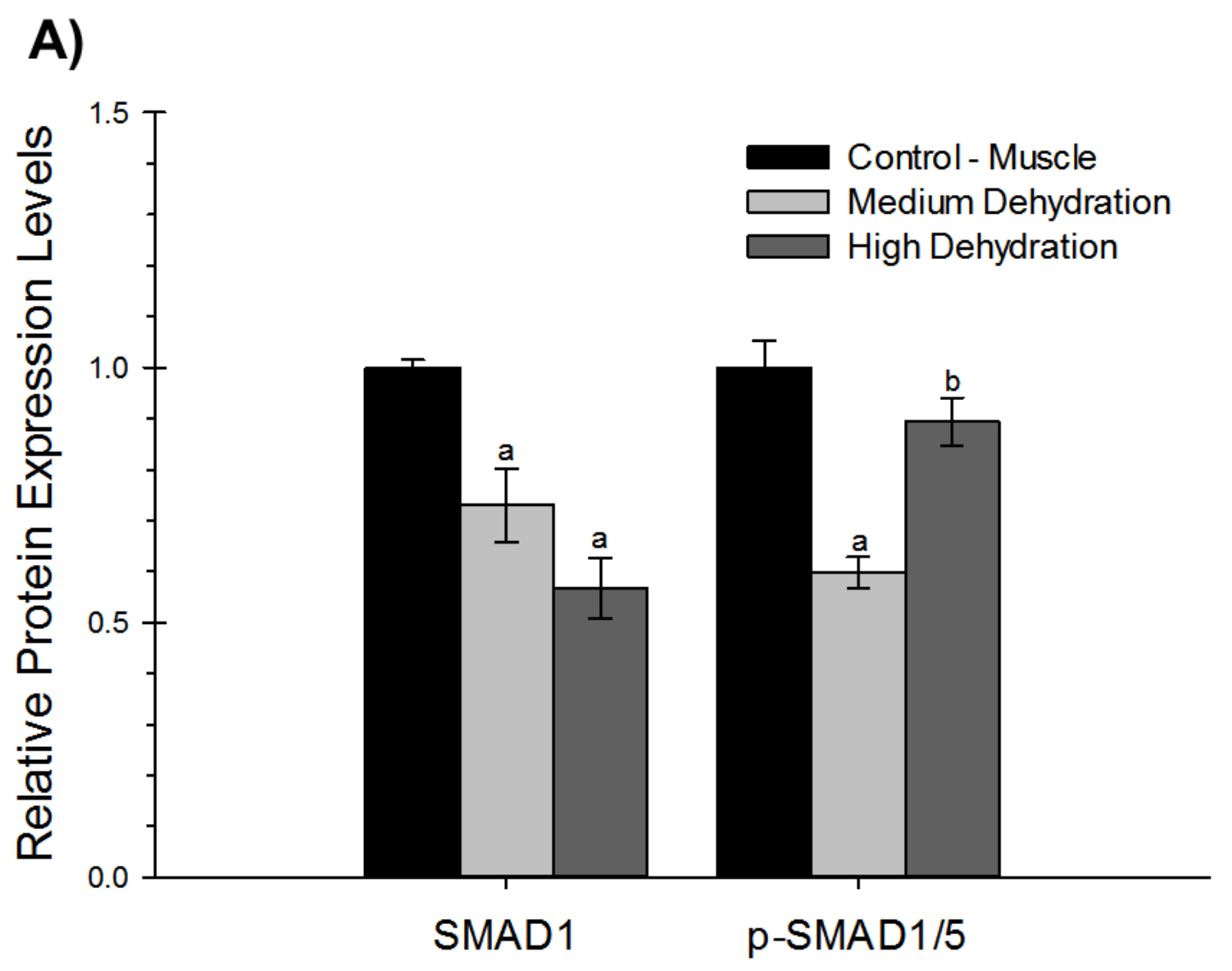

B)

\section{SMAD1}

\section{p-SMAD1/5}

Figure 3.6 - Effects of medium and high dehydration on the protein expression of SMAD1 (52 kDa) and phospho-SMAD1/5 (serine 463/465) in African clawed frog muscle as determined by immunoblotting. A histogram of normalized mean protein levels (A) is shown along with representative immunoblots (B). Data are means \pm S.E.M., $n=3-4$ independent trials. Significantly different from the corresponding ( $a$ ) control or $(b)$ medium dehydration values using a one-way ANOVA and a Tukey's post-hoc test, $p<0.05$. 


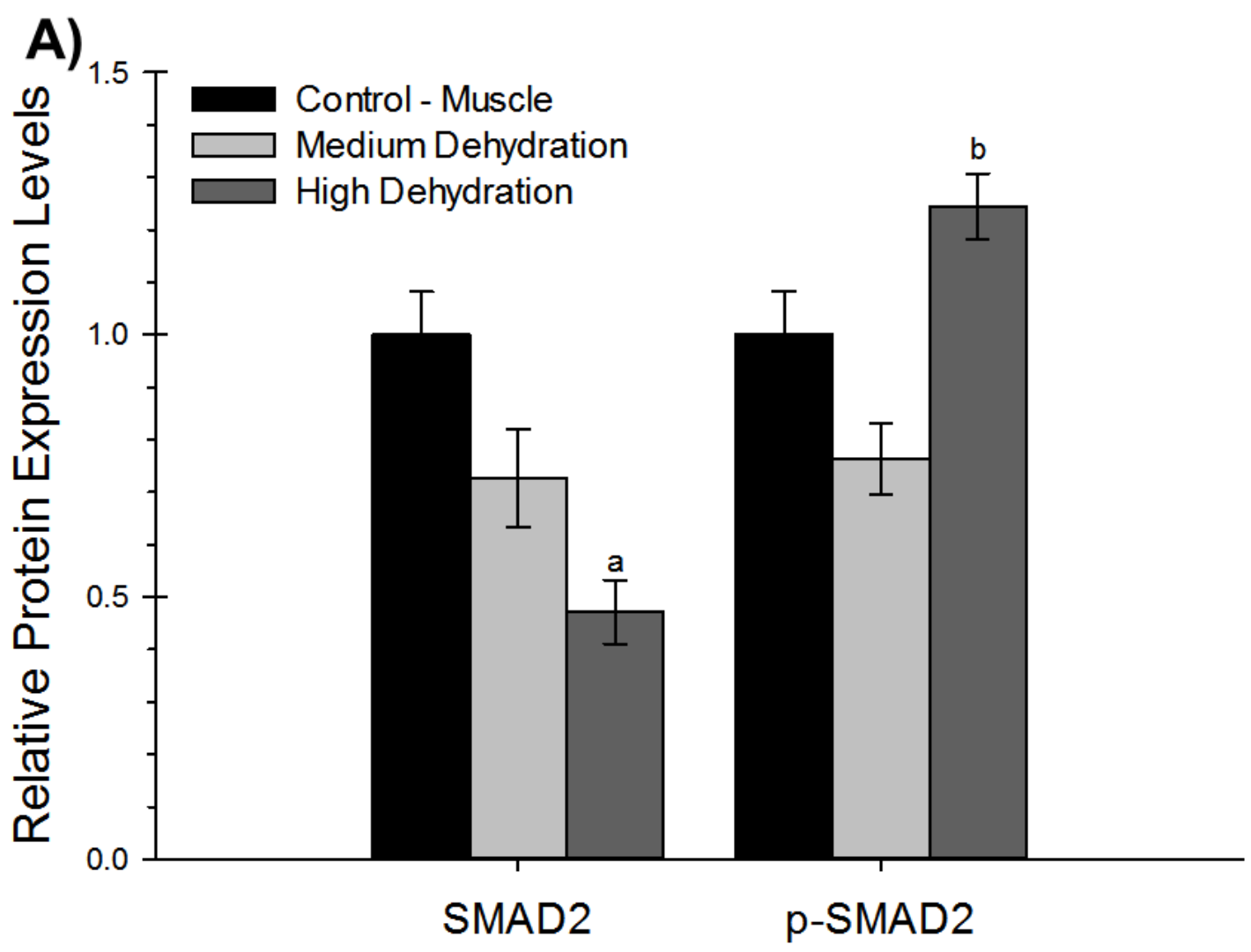

B)

SMAD2

p-SMAD2
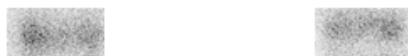

Figure 3.7 - Effects of medium and high dehydration on the protein expression of SMAD2 (52 kDa) and phospho-SMAD2 (serine 465/467) in African clawed frog muscle as determined by immunoblotting. A histogram of normalized mean protein levels (A) is shown along with representative immunoblots $(\mathbf{B})$. Data are means \pm S.E.M., $n=3-4$ independent trials. Significantly different from the corresponding (a) control or $(b)$ medium dehydration values using a one-way ANOVA and a Tukey's post-hoc test, $p<0.05$. 
A)

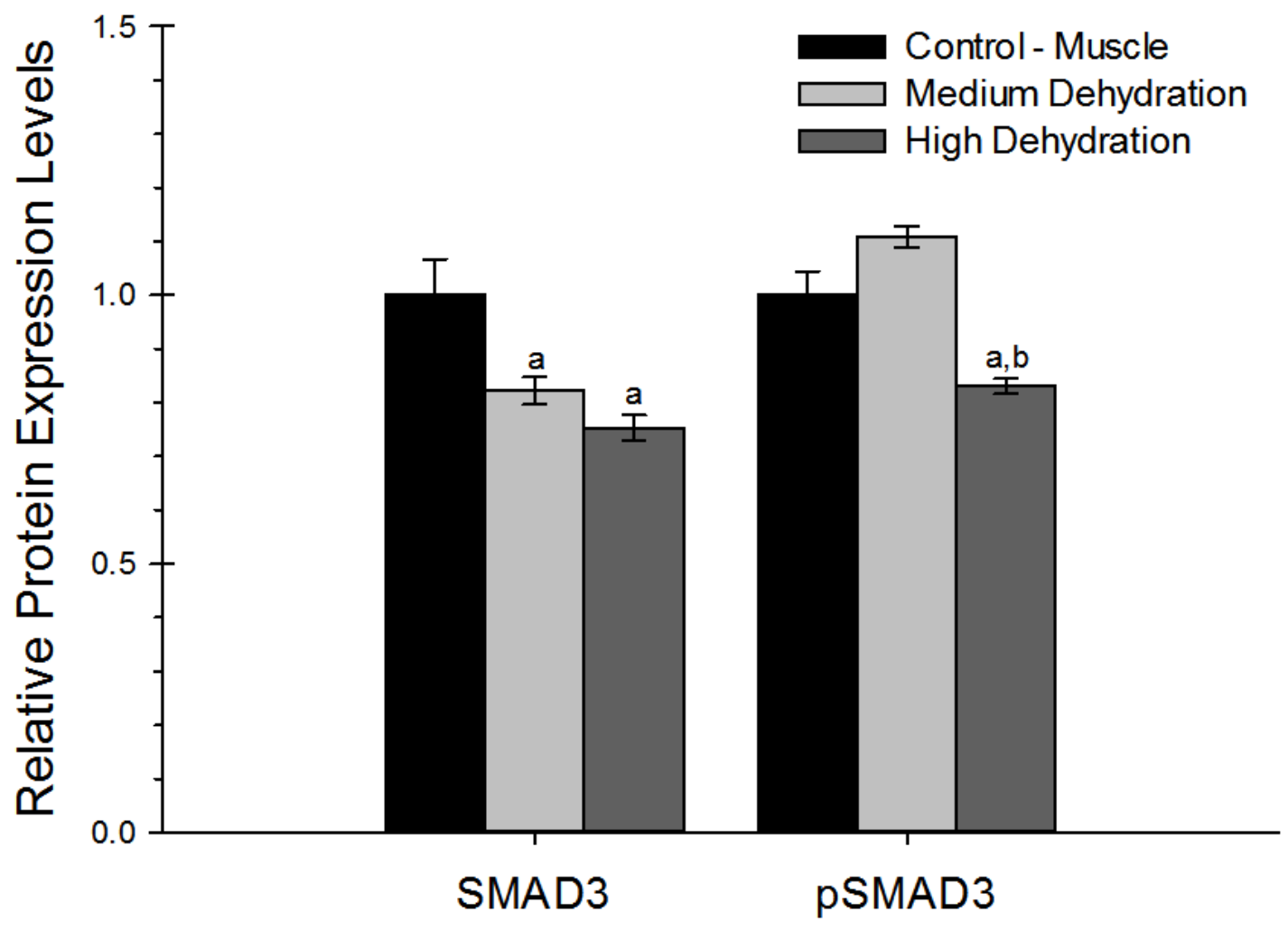

B)

\section{SMAD3}

\section{p-SMAD3}
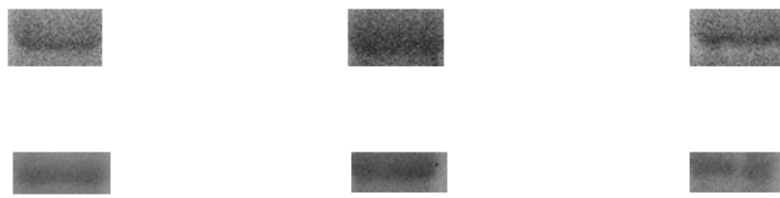

Figure 3.8 - Effects of medium and high dehydration on the protein expression of SMAD3 (48 kDa) and phospho-SMAD3 (serine 423/425) in African clawed frog muscle as determined by immunoblotting. A histogram of normalized mean protein levels (A) is shown along with representative immunoblots $(\mathbf{B})$. Data are means \pm S.E.M., $n=3-4$ independent trials. Significantly different from the corresponding $(a)$ control or $(b)$ medium dehydration values using a one-way ANOVA and a Tukey's post-hoc test, $p<0.05$. 
A)

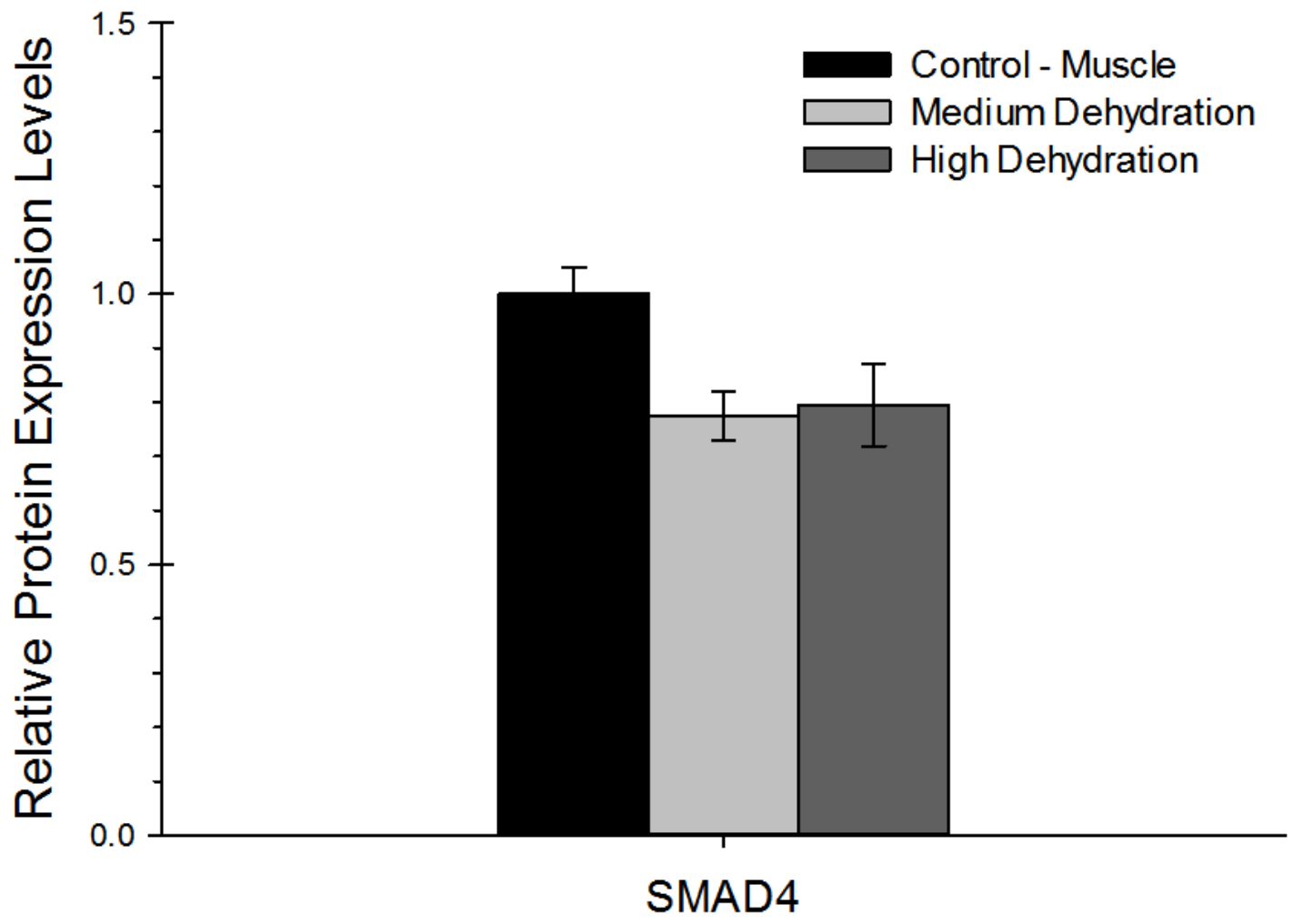

B)

\section{SMAD4}

Figure 3.9 - Effects of medium and high dehydration on the protein expression of SMAD4 (60 kDa) in African clawed frog muscle as determined by immunoblotting. A histogram of normalized mean protein levels (A) is shown along with representative immunoblots (B). Data are means \pm S.E.M., $n=3-4$ independent trials. Significantly different from the corresponding $(a)$ control or $(b)$ medium dehydration values using a one-way ANOVA and a Tukey's post-hoc test, $p<0.05$. 
A)

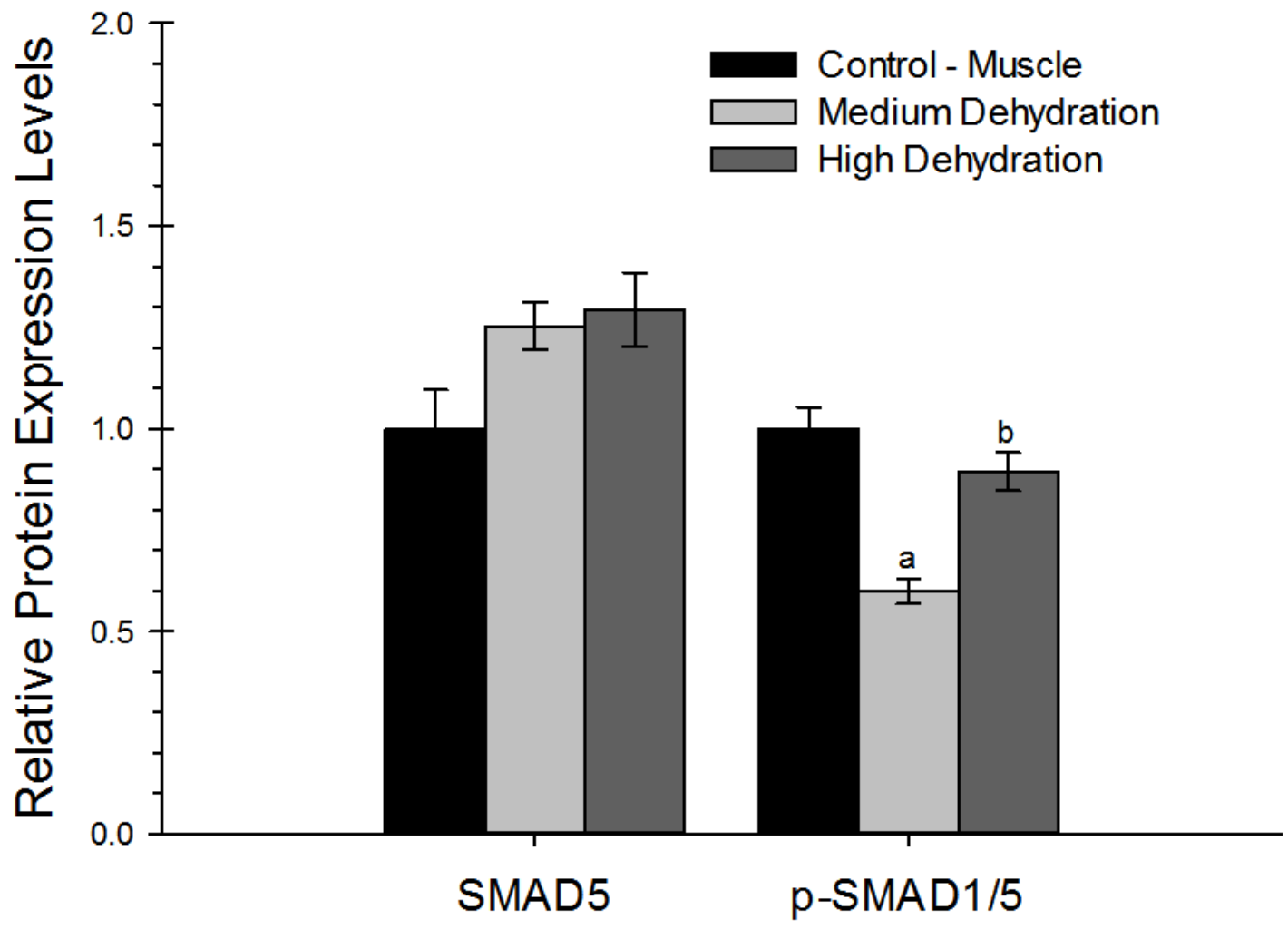

B)

SMAD5
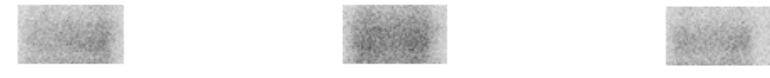
p-SMAD1/5
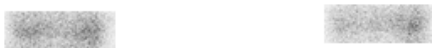

Figure 3.10 - Effects of medium and high dehydration on the protein expression of SMAD5 (52 kDa) and phospho-SMAD1/5 (serine 463/465) in African clawed frog muscle as determined by immunoblotting. A histogram of normalized mean protein levels (A) is shown along with representative immunoblots $(\mathbf{B})$. Data are means \pm S.E.M., $n=3-4$ independent trials. Significantly different from the corresponding (a) control or $(b)$ medium dehydration values using a one-way ANOVA and a Tukey's post-hoc test, $p<0.05$. 


\subsection{3 - Downstream Targets of the TGF- $\beta$ Pathway in Liver}

Expression of TGF- $\beta$ precursor proteins decreased significantly in liver under dehydration conditions; relative levels were reduced in medium dehydration to $72 \%$ of the control values and to $63 \%$ under high dehydration (MD: $0.723 \pm 0.047, p<0.05$; HD: 0.627 $\pm 0.051, p<0.01)$ (Figure 3.11). Levels of c-Myc protein expression were also suppressed in the liver during dehydration; c-Myc dropped to $45 \%$ of controls during medium desiccation and remained the same level (48\% of controls) during high dehydration (MD: $0.447 \pm 0.030, p<0.01$; HD: $0.479 \pm 0.046, p<0.01$ ) (Figure 3.12). However, relative phosphorylation increased very strongly with p-c-Myc being expressed at $506 \%$ of its control levels under medium dehydration, while under high desiccation stress the liver expressed the phosphorylated form at 796\% of controls (MD: $5.061 \pm 0.626, p<0.05$; HD: $7.961 \pm 0.663, p<0.001)$ (Figure 3.12). There was also a significant difference detected in phosphorylated protein expression levels between the two dehydration conditions $(p<0.001)$. c-Fos protein levels in the liver increased to 1.64 -fold over control levels during medium dehydration, and were maintained at 1.57 -fold under high dehydration conditions (MD: $1.643 \pm 0.051, p<0.01$; HD: $1.568 \pm 0.133, p<0.05$ ) (Figure 3.13). When phosphorylated, however, p-c-Fos was at its highest during medium dehydration (MD: $1.576 \pm 0.155, p<0.05 ;$ HD: $0.872 \pm 0.115$, N.S.). Phospho-c-Fos levels differed significantly between stress conditions, dropping by $70 \%$ from medium to high dehydration $(p<0.05)$. 


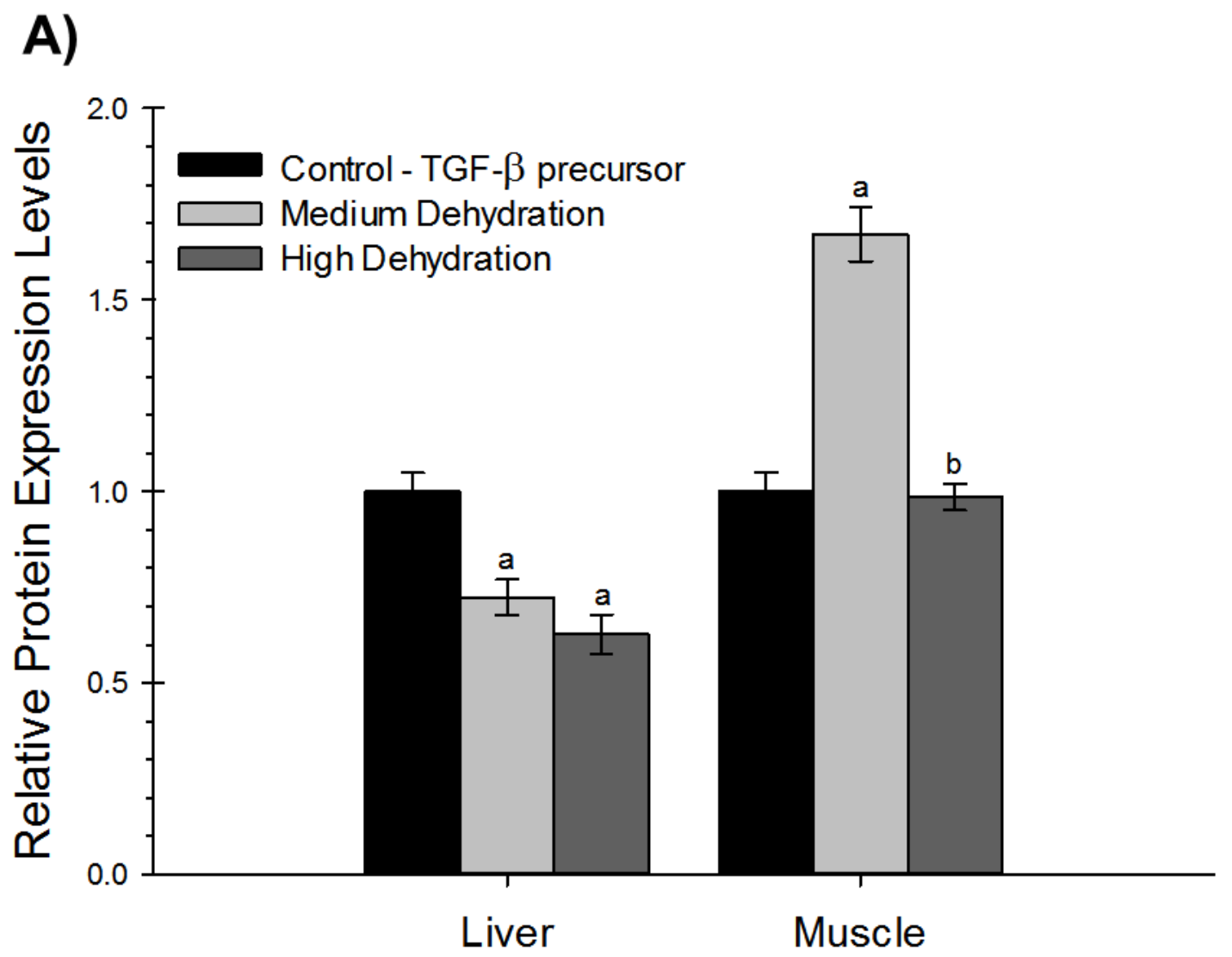

B)

\section{Liver \\ Muscle}
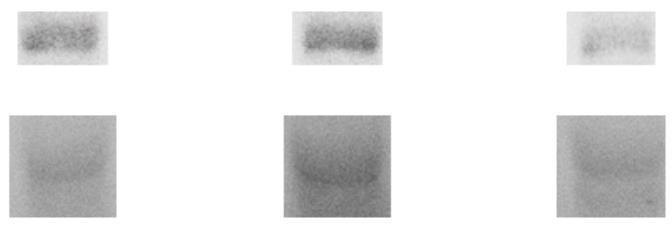

Figure 3.11 - Effects of medium and high dehydration on the protein expression of TGF- $\beta$ precursor (45 $\mathrm{kDa}$ ) in African clawed frog liver and muscle as determined by immunoblotting. A histogram of normalized mean protein levels (A) is shown along with representative immunoblots $(\mathbf{B})$. Data are means \pm S.E.M., $n=3-4$ independent trials. Significantly different from the corresponding $(a)$ control or $(b)$ medium dehydration values using a one-way ANOVA and a Tukey's post-hoc test, $p<0.05$. 


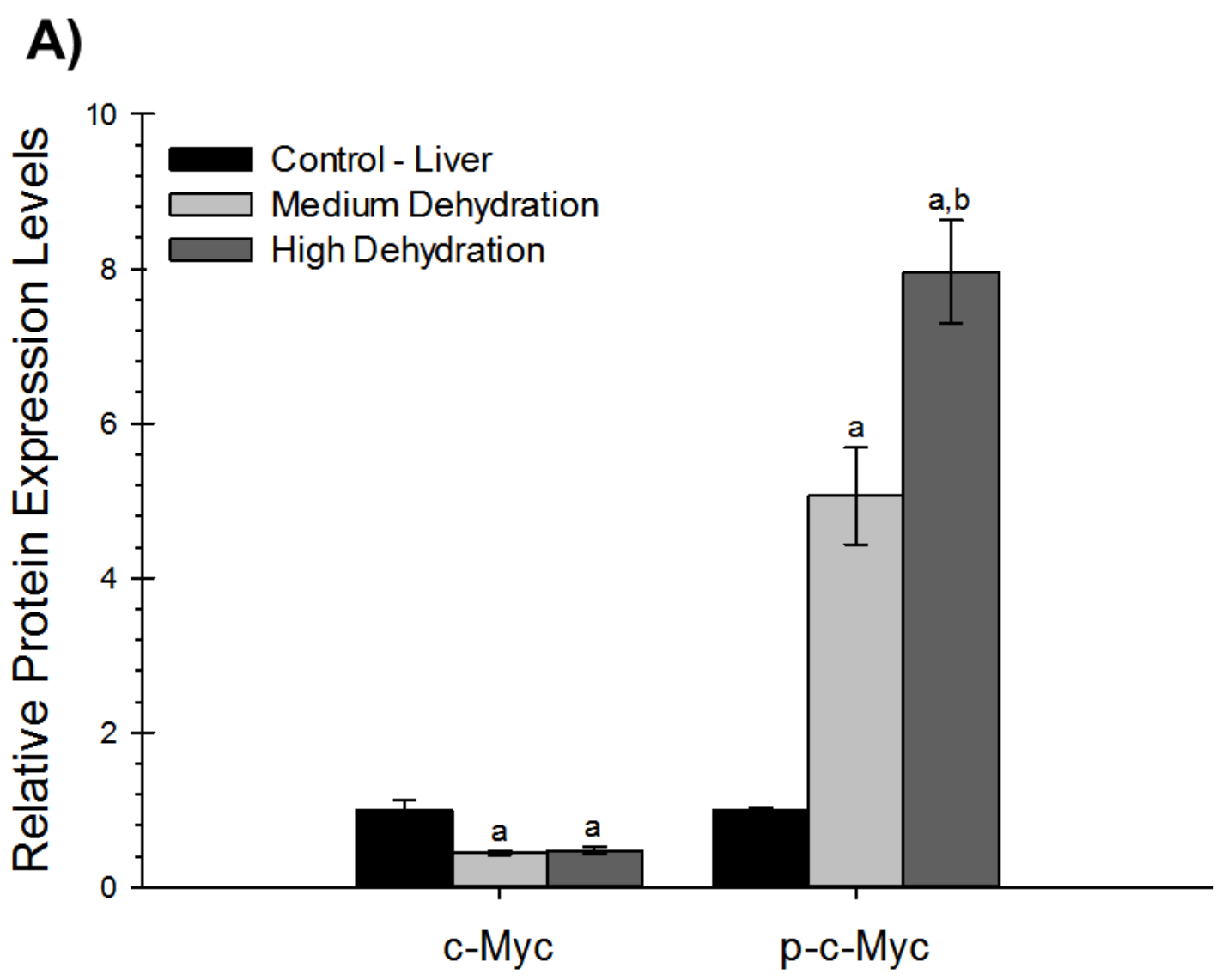

B)

\section{c-Myc p-c-Myc}

Figure 3.12 - Effects of medium and high dehydration on the protein expression of total c-Myc (47 kDa) and phospho-c-Myc (threonine 58/serine 62) in African clawed frog liver as determined by immunoblotting. A histogram of normalized mean protein levels (A) is shown along with representative immunoblots (B). Data are means \pm S.E.M., $n=3-4$ independent trials. Significantly different from the corresponding $(a)$ control or $(b)$ medium dehydration values using a one-way ANOVA and a Tukey's post-hoc test, $p<0.05$. 


\section{A)}

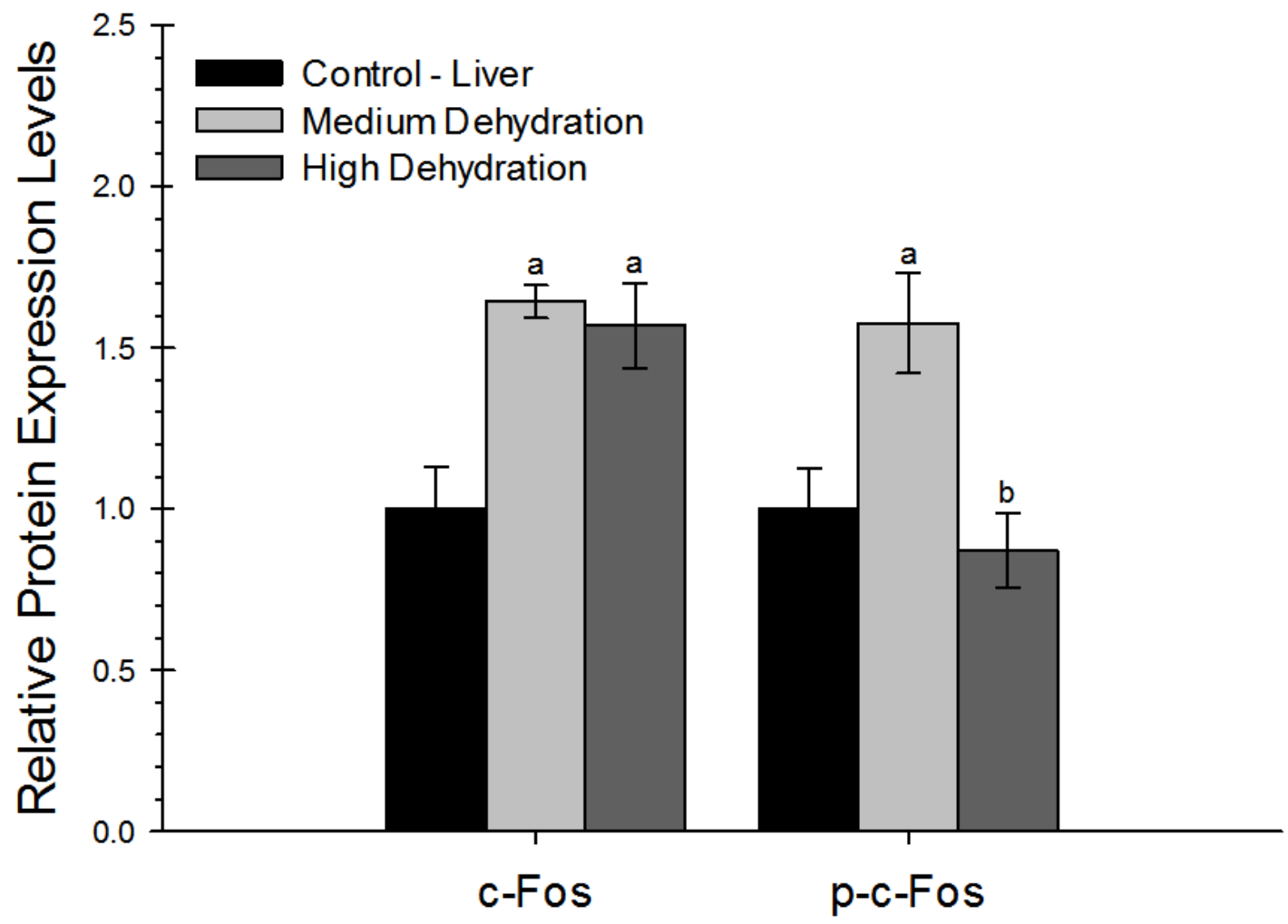

B)

\section{c-Fos}

p-c-Fos
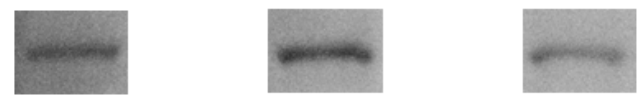

Figure 3.13 - Effects of medium and high dehydration on the protein expression of total c-Fos (40kDa) and phospho-c-Fos (threonine 232) in African clawed frog liver as determined by immunoblotting. A histogram of normalized mean protein levels $(\mathbf{A})$ is shown along with representative immunoblots $(\mathbf{B})$. Data are means \pm S.E.M., $n=3-4$ independent trials. Significantly different from the corresponding $(a)$ control or $(b)$ medium dehydration values using a one-way ANOVA and a Tukey's post-hoc test, $p<$ 0.05 . 


\subsection{4 - Downstream Targets of the TGF- $\beta$ Pathway in Muscle}

In skeletal muscle, TGF- $\beta$ precursor protein levels were similar in the control and high dehydration conditions, but increased by 1.67 -fold in medium dehydration as compared to control muscle (MD: $1.671 \pm 0.070, p<0.001$; HD: $0.986 \pm 0.035$, N.S.) (Figure 3.11). Levels of c-Myc protein were not different during medium dehydration (56\%) when compared to control frogs, and subsequently rose to $128 \%$ of control levels under high dehydration conditions (MD: $0.559 \pm 0.047$, N.S.; HD: $1.278 \pm 0.175$, N.S.) (Figure 3.14); while the protein level was not significant compared to controls it was significantly different from medium dehydration as determined by Tukey's post-hoc testing $(p<0.01)$. Levels of the phosphorylated form of c-Myc rose strongly under high dehydration (MD: $1.421 \pm 0.184$, N.S.; HD: $2.474 \pm 0.213, p<0.01)$. As in the liver, the expression levels of p-c-Myc between the dehydration levels were also significantly different $(p<0.01)$. In $X$. laevis muscle, c-Fos protein expression was 1.44-fold of its control levels under medium dehydration, and rose to 2.91-fold of control levels under high dehydration (MD: $1.442 \pm$ 0.097, N.S.; HD: $2.911 \pm 0.118, p<0.001)$; this change was also significantly different from medium dehydration $(p<0.001)$ (Figure 3.15). Phosphorylation of c-Fos increased significantly under medium dehydration and returned to levels similar to control (MD: $1.589 \pm 0.081, p<0.005 ;$ HD: $0.907 \pm 0.058$, N.S.). Significant differences were detected between MD and HD frogs $(p<0.005)$. 
A)

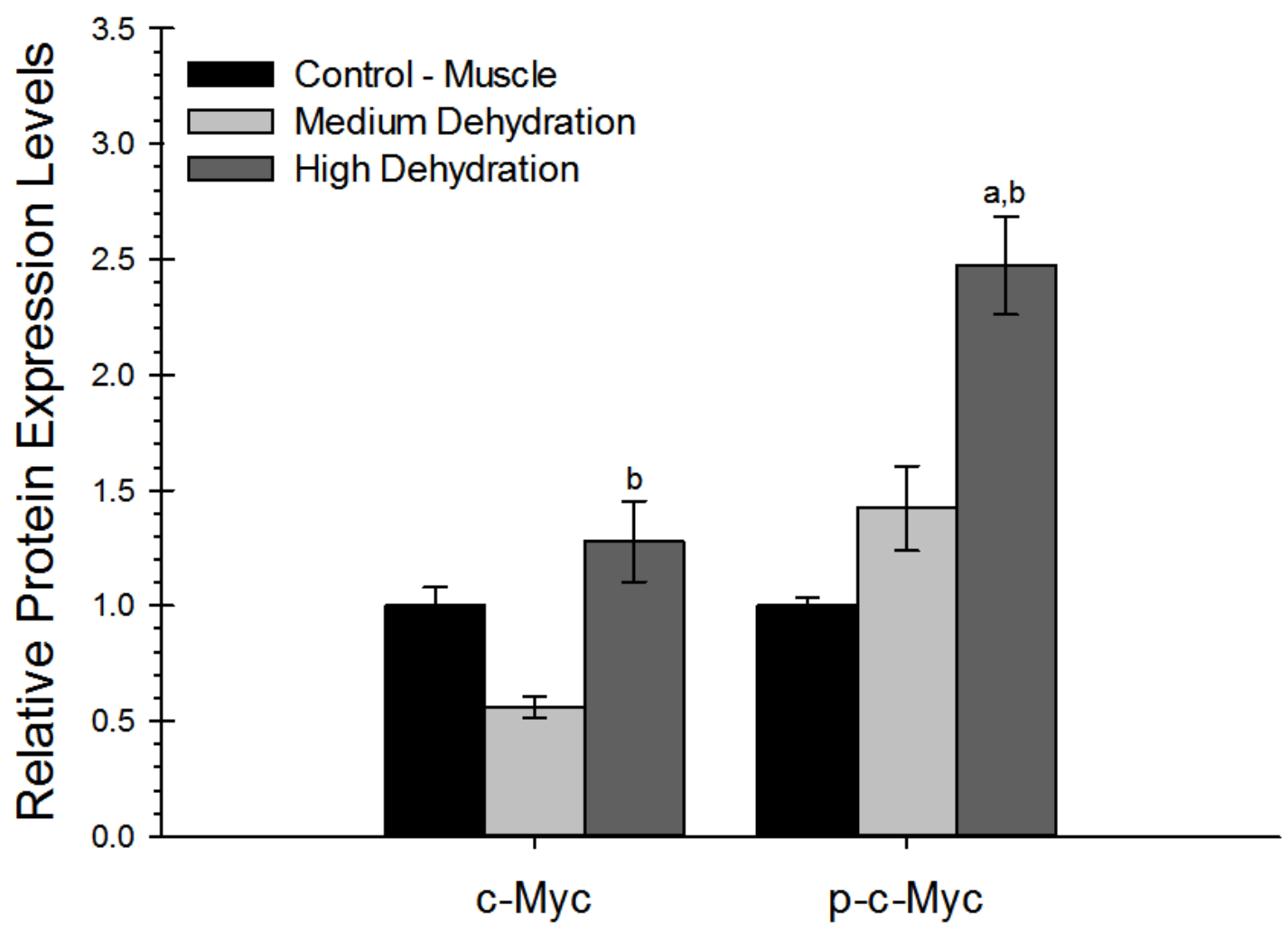

B)

\section{c-Myc}

p-c-Myc
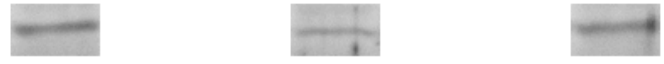

Figure 3.14 - Effects of medium and high dehydration on the protein expression of total c-Myc (47 kDa) and phospho-c-Myc (threonine 58/serine 62) in African clawed frog muscle as determined by immunoblotting. A histogram of normalized mean protein levels (A) is shown along with representative immunoblots (B). Data are means \pm S.E.M., $n=3-4$ independent trials. Significantly different from the corresponding (a) control or $(b)$ medium dehydration values using a one-way ANOVA and a Tukey's post-hoc test, $p<0.05$. 


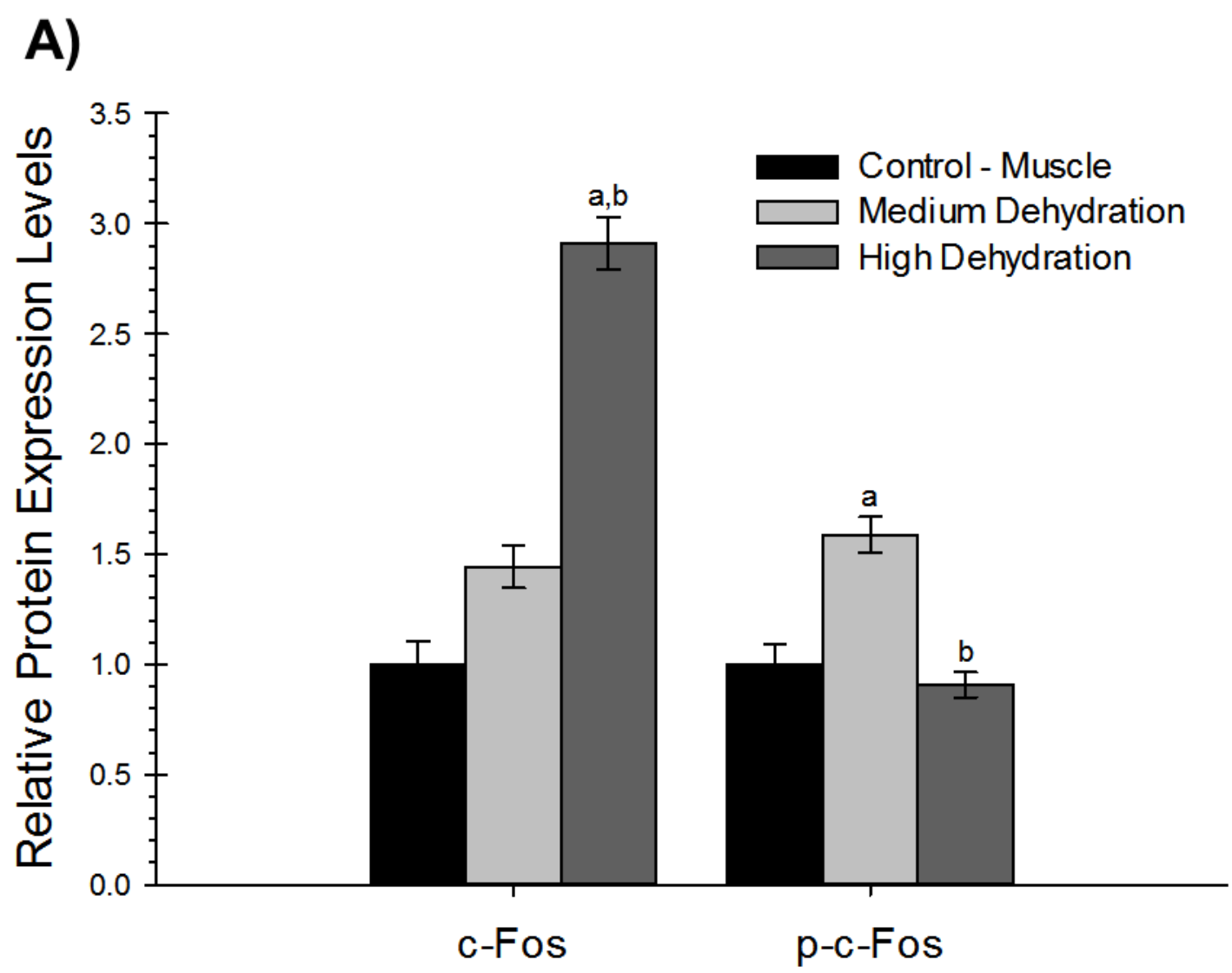

B)

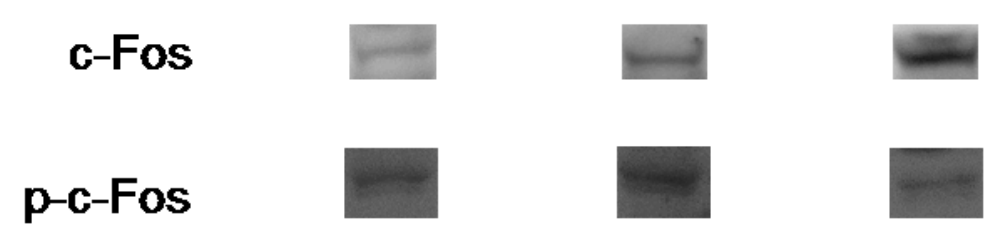

Figure 3.15 - Effects of medium and high dehydration on the protein expression of total c-Fos (40 kDa) and phospho-c-Fos (threonine 232) in African clawed frog muscle as determined by immunoblotting. A histogram of normalized mean protein levels $(\mathbf{A})$ is shown along with representative immunoblots $(\mathbf{B})$. Data are means \pm S.E.M., $n=3-4$ independent trials. Significantly different from the corresponding (a) control or $(b)$ medium dehydration values using a one-way ANOVA and a Tukey's post-hoc test, $p<0.05$. 


\section{4 - Discussion}

\subsection{1 - X. laevis Tissues Cease Cell Cycle during Dehydration}

In previous studies of Xenopus laevis, metabolic rate depression was examined in several contexts, one of them being cell cycle arrest. Phosphorylation was observed to play a major role in suppressing protein translation and activating cell cycle inhibitors such as $\mathrm{p} 21^{\mathrm{WAF} 1 / \mathrm{Cip} 1}(\mathrm{p} 21)$ and $\mathrm{p} 27^{\mathrm{Kip} 1}$ (p27) in the dehydrating anurans (Luu, 2013). Additionally, it is known that animals adapting to extreme environmental stresses such as dehydration use cell cycle arrest as a means of conserving energy as it is one of the main energy consuming processes under normal conditions; as seen in Figure 1.2 (Storey, 2012). The wood frog (Rana sylvatica), for example, while experiencing freezing conditions showed typical signs of cell cycle arrest at the molecular level, and a reversal of these signs during recovery from a frozen state showing a correlation between stress and cell cycle regulation (Zhang and Storey, 2012). Similarly in hibernating thirteen-lined ground squirrels, a reduction in specific cyclin proteins along with upregulation of cyclin dependent kinase (CDK) inhibitors implicated cell cycle suppression as a mechanism of survival during periods of torpor (Wu and Storey, 2012). The SMAD signaling pathway has been implicated in the induction of $\mathrm{p} 21$ expression, with the caveat that cell growth arrest also depends on the regulation of additional gene targets (Pardali et al., 2005; Siegel and Massagué, 2003).

In liver of dehydrated $X$. laevis, SMAD3 and phosphorylated SMAD1/5 protein expression were suppressed during high dehydration (Figures 3.1, 3.3, 3.5) whereas the other SMAD proteins remained virtually unchanged (Figures 3.2, 3.4) (except for SMAD1 protein upregulation during medium dehydration; Figure 3.1). TGF- $\beta$ precursor protein, an indicator of the levels of the matured signaling cytokines TGF- $\beta$ I, II, and III, also 
exhibited downregulation and in fact mimicked the pattern observed for SMAD3 (Figure 3.11). Taken together, this is evidence that the CDK inhibitors downstream of the pathway would be differentially phosphorylated so that they may disrupt cell division and proliferation. This mode of action has been established using hematopoietic, epithelial, and endothelial cell types (Siegel and Massagué, 2003). Typically, SMAD4 follows the trend of the R-SMADs because it binds to them to form dimers and subsequently the trimeric complex is shuttled off to the nucleus. Therefore, when R-SMADs become differentially expressed within the cell, SMAD4 should be proportionally regulated up or down for efficient function of the signaling pathway. This was not the case in X. laevis liver as SMAD4 levels did not change in any significant way to control. Nevertheless, p21 regulation by TGF- $\beta$ has been observed in other studies to operate independently of SMAD4 expression (Figure 3.4) (Ijichi et al., 2004).

In muscle, similar patterns of SMAD expression were observed. R-SMADs, and their phosphorylated counterparts, were more globally suppressed and to a greater extent during high dehydration (Figures 3.6 - 3.8), except SMAD5. However, CDK inhibitors p21 and p27 were suppressed by $60 \%$ meaning that cell cycle progression is facilitated in the muscle tissues of X. laevis (Luu, 2013).

In addition to differential SMAD3 protein expression, c-Myc was expressed at lower levels during dehydration in $X$. laevis liver and muscle (Figures 3.12 and 3.14). The expression levels of total c-Myc in liver dropped to $45-48 \%$ of controls during medium and high dehydration (Figure 3.12). In muscle, a similar trend occurred where c-Myc expression dropped by nearly half of the control level during medium dehydration, but returned to levels similar to controls during high dehydration (Figure 3.14). c-Myc is a 
well-known inhibitor of the cell cycle kinase inhibitors p21 and p15, meaning it is a procell cycle protein (Feng et al., 2002; Pardali et al., 2005; Hirschhorn et al., 2012; Shaulian and Karin, 2001). With its suppression there is also an associated downregulation in activated cell cycle proteins. Interestingly, the phosphorylated form of c-Myc was observed to increase dramatically in response to dehydration. This trend been connected to protective roles to compensate for the oxidative stress concomitant with hypometabolism (Benassi et al., 2006).

\subsection{2 - X. laevis Tissues Experience Oxidative Stress under Dehydration}

The African clawed frog employs hypometabolism as a strategy of survival when faced with dehydration (Storey, 2012). This strategy is not without its own accompanying stresses that an organism must endure and overcome. However the anuran has developed responses to combat these side-effects and can easily endure hypometabolism and desiccation for extended periods of time. In previous studies, yeast (Saccharomyces cerevisiae) cells undergoing dehydration showed a 10-fold increase in oxidation linking the loss of water to oxidative stress; similarly as the frog desiccates its respiration slows and oxidative stress becomes of concern (Pereira et al., 2003). Despite this connection, studies have not to date been able to elucidate the source of free radical production during dehydration although free radicals have been implicated as one of the major culprits in desiccation injury (Hansen et al., 2006). Anurans could exhibit peroxidation of their main fuel source (lipids), and damage to proteins and nucleic acids with severe consequences on metabolism as a result of the formation of ROS species under these conditions if not for the evolutionarily developed pathways designed to counteract oxidative stress (Hansen et al., 2006). To protect against these forms of chemical damage, cells possess antioxidant defense mechanisms that include both enzymes (peroxidases, catalases, and superoxide 
dismutases) and metabolites (vitamins D and E, and glutathione (GSH), among others) (Jamieson, 1998; Pereira et al., 2003). The difference in the stoichiometric mole ratio between reactive oxygen species (ROS) equivalents being produced per unit time and antioxidant cell defenses determines the degree of oxidative stress felt by cells, and by extension the organism (França et al., 2007).

As mentioned previously in 3.4.1, p-c-Myc was observed to be intensely upregulated in X. laevis liver (5-fold increase in MD, and 8-fold increase in HD, Figure 3.12) as well as a 2.5-fold increase in muscle under HD (Figure 3.14). In its phosphorylated form c-Myc has an important role in protecting cells and tissues from ROS (Benassi et al., 2006). Phospho-c-Myc transcriptionally regulates $\gamma$-glutamyl-cysteine-synthetase ( $\gamma$-GCS), the rate-limiting enzyme used in GSH biosynthesis. GSH plays crucial roles in cellular detoxification, redox balance, and the stress response, and is regarded as the most important low molecular weight thiol (Deneke and Fanburg, 1989; Blokhina et al., 2003). This mechanism of action requires us to reexamine the simplified version of oxidative stress as an imbalance of prooxidants and antioxidants that could lead to oxidative apoptosis through an excess of ROS (Hockenbery et al., 1993; Blokhina et al., 2003). However, mediators of oxidative stress can also function as second messengers in signal transduction (Hansen et al., 2006). Their signaling capabilities are poorly understood in terms of their specificity and how this occurs without incurring damage. However, hydrogen peroxide $\left(\mathrm{H}_{2} \mathrm{O}_{2}\right)$, a common ROS, enhances the recruitment of c-Myc to the $\gamma$-GCS promoter. The upregulation of $\gamma$-GCS, both heavy and light subunits, leads to an increase in GSH concentrations because it is biosynthetically produced in cells at a rapid pace (Benassi et al., 2006; Deneke and Fanburg, 1989, Biroccio et al., 2004). The phosphorylation of c- 
Myc at Ser62 (via the ERK/Ras pathway) not only promotes this process, but is a requirement for recruitment to $\gamma$-GCS promoters and governs the cellular response to oxidative stress induced by different stimuli (Benassi et al., 2006). Phosphorylation of cMyc, therefore, is critical in regulating oxidative stress in the cells of the frog and mediating ROS stemming from dehydration.

FOXOs are a set of proteins that are known to play a major role in regulating the expression of antioxidant genes in $X$. laevis while the frog undergoes seasonal dehydration (Malik and Storey, 2009, 2011). Indeed, FoxO1 protein levels increased significantly in the hepatocyte nucleus and binding to DNA to affect the transcription of important redox enzymes. MnSOD protein and transcript levels were raised 1.4- and 1.5-fold, respectively, in liver while catalase protein was upregulated in both muscle and liver, with liver also displaying a greater than twofold elevation in transcript levels compared to control frogs (Malik, 2009). This evidence confirms the important role that SMAD transcription factors play in switching on the oxidative stress response in muscle and liver.

\subsection{3 - AP-1 Proteins and Their Role in X. laevis Tissues}

While oxidative stress and its mediation play major roles in the liver of the anuran, the regulation of hypertrophy/atrophy, regenerative, and apoptotic pathways play critical roles in the muscle (Oshitari et al., 2002; Shaulian and Karin, 2001). SMADs 3 and 4 have been shown to physically interact with AP-1 proteins (c-Fos and c-Jun) and activate their transcriptional activity (Liberati et al., 1999). Although SMADs do not interact directly with AP-1 DNA binding sites, they associate with c-Jun and rapidly phosphorylate it in response to cytokine signaling by TGF- $\beta$ (Liberati et al., 1999). c-Fos was upregulated in X. laevis liver under both dehydration conditions (about a 1.6-fold increase in protein levels compared to CXL, Figure 3.13), whereas muscle showed a similar pattern with a 1.5 -fold 
increase during MD, but a more dramatic upturn during HD (nearly a three-fold increase compared to control, Figure 3.15). c-Jun was upregulated during high dehydration in both liver and muscle in about the same proportions (about a 1.5 -fold increase compared to CXL; Malik, 2009). Both of these DNA binding proteins are merely part of the Fos and Jun subgroups, respectively, of transcription factors that form AP-1 dimers when activated. Their activation is reliant on many forms of physiological and pathological stimuli and in the case of X. laevis that stimulus or stress is dehydration (Shaulian and Karin, 2001). AP1 proteins can be both heterodimers and homodimers made up of the basic region-leucine zipper (bZIP) proteins mentioned earlier (Fos and Jun family proteins). While Jun:Jun homodimers are stable and can bind TPA recognition/response elements (TREs: 5'TGAG/CTCA-3'), Fos proteins do not form stable homodimers. Instead Fos and Jun proteins can combine to form Fos:Jun dimers which are actually more stable than Jun:Jun dimers (Halazonetis et al., 1988; Kouzarides and Ziff, 1988). These complexes also regulate the expression of cell cycle regulators such as p16, p19 ${ }^{\mathrm{ARF}}, \mathrm{p} 21, \mathrm{p} 53$, and Cyclin D1. In previous work Cyclin D expression levels were shown to be significantly higher in $X$. laevis liver during MD (2.5-fold), but its nuclear levels were suppressed during HD (about $80 \%$ of CXL levels) as well as a decrease for the phosphorylated form $(80 \%$ of CXL levels) was observed (Luu, 2013). From this it can be seen that AP-1 proto-oncoproteins, c-Fos and c-Jun, could be possibly aiding in further inducing cell cycle arrest in the liver and muscle of the frog under dehydration stress.

The phosphorylation of AP-1 proteins further amplifies their transcriptional activity, while sumoylation (another form of posttranslational modification) attenuates the activity of the heterodimer, especially in the case of c-Fos (Shaulian and Karin, 2001; Bossis et al., 
2006). Interestingly, phosphorylation of c-Fos at Thr232 not only prevents sumoylation, but superactivates it (Bossis et al., 2006). This phosphorylation was observed to be upregulated in anuran liver and muscle during medium dehydration by 1.5 -fold as compared to control (Figures 3.13 and 3.15). The lesser known ability of AP-1 proteins is their capability to promote DNA repair, because oxidative injury is experienced by the frog's tissues during dehydration. DNA synthesis and repair is stimulated by these proteins in response to the accumulation of ROS (Tanos et al., 2005; Monje et al., 2003). So, as one can see, c-Fos and c-Jun can play important roles in the processes that control muscle mass, apoptosis, and most importantly DNA repair mechanisms.

\section{$\underline{3.4 .4-\text { Discussion Figures }}$}
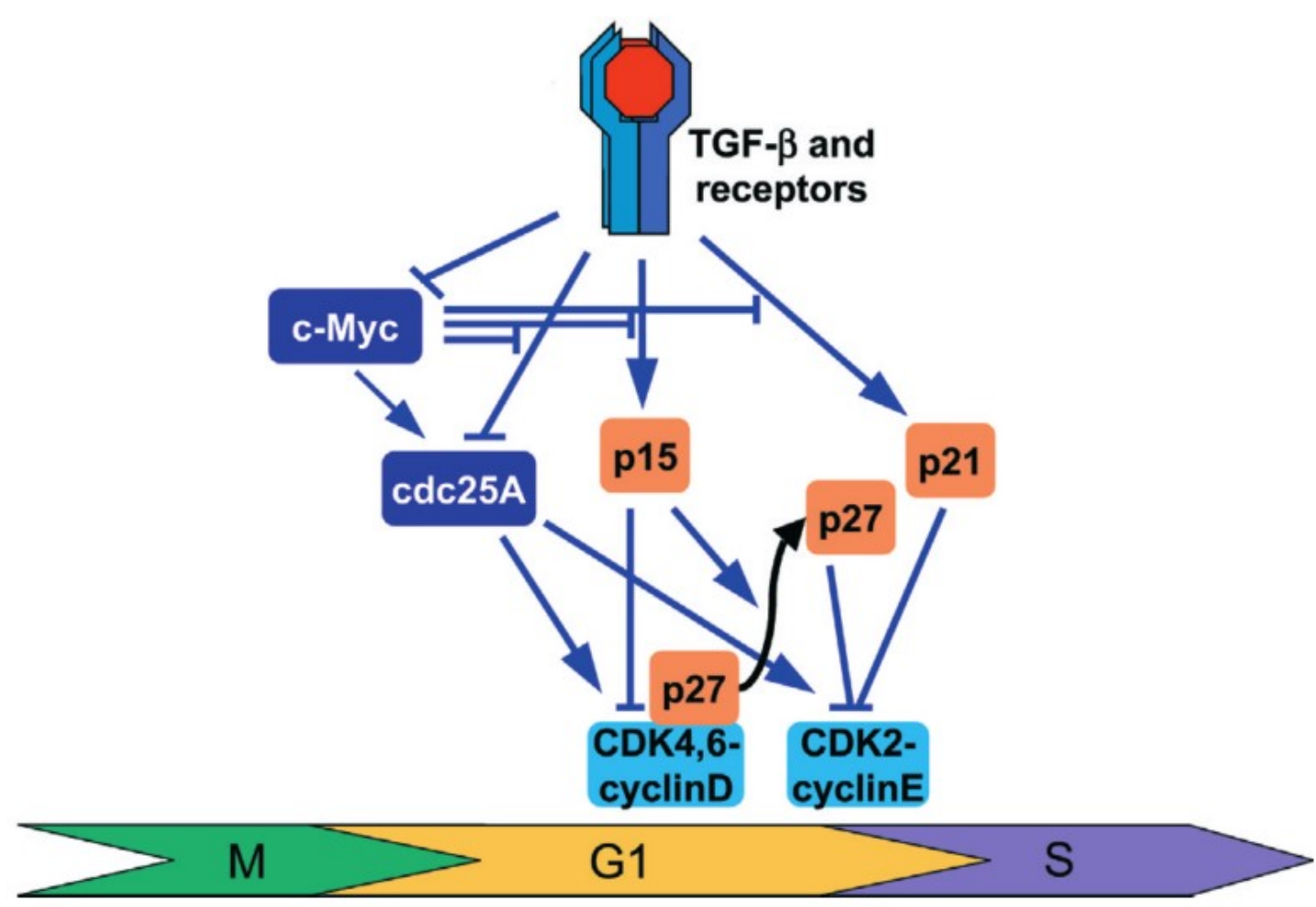

Figure 3.16 - Gene regulation in TGF- $\beta$-induced cell cycle arrest; from ten Dijke et al. (2002). 


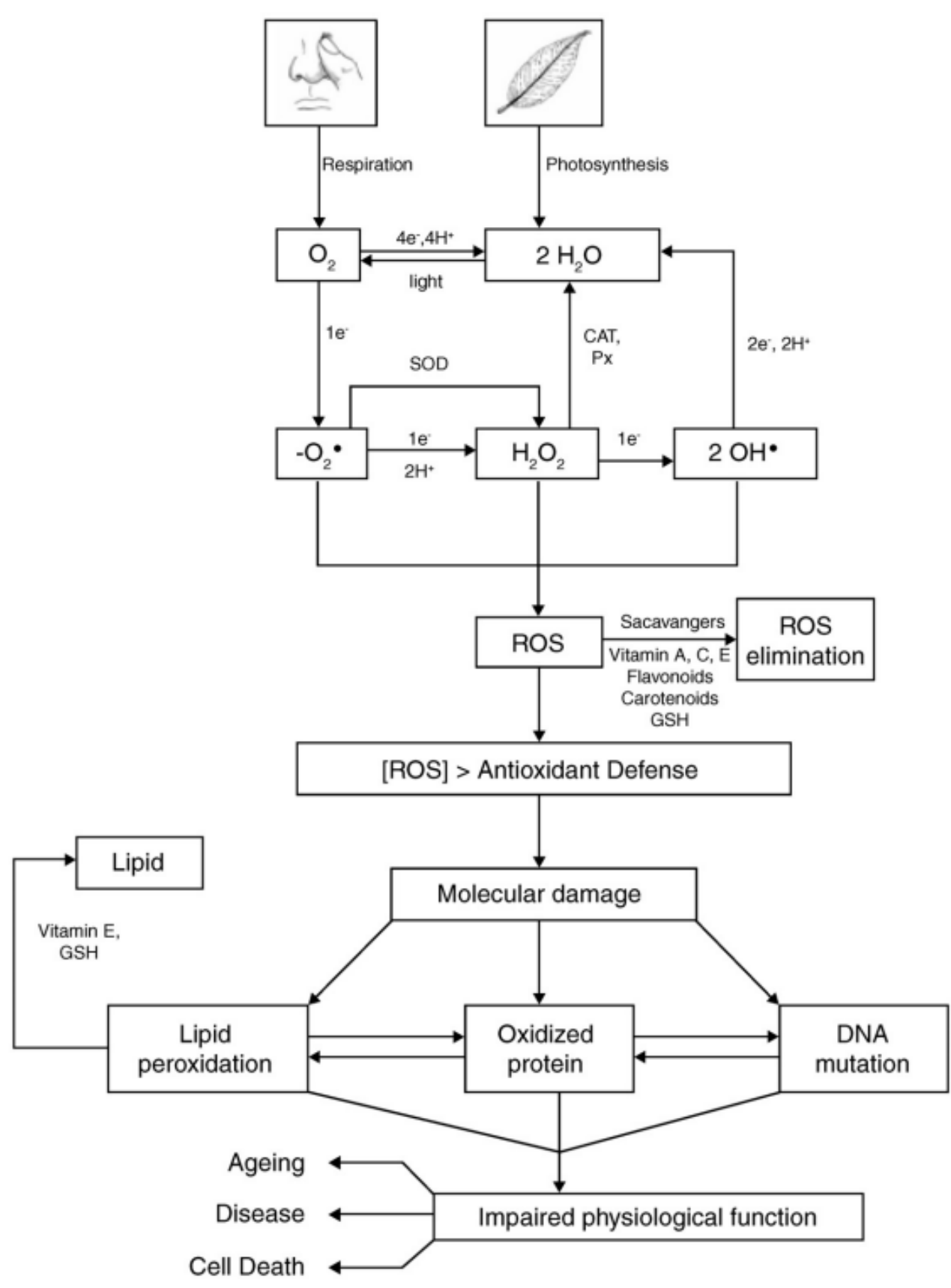

Figure 3.17 - The sources and cellular responses to ROS. A sophisticated enzymatic and non-enzymatic antioxidant defense system is shown which include such redox enzymes and molecules as catalase (CAT), superoxide dismutase (SOD), peroxidases (Px) and glutathione (GSH). These species counteract and regulate overall ROS levels within the cells to maintain physiological homeostasis of the tissues; from França (2007). 


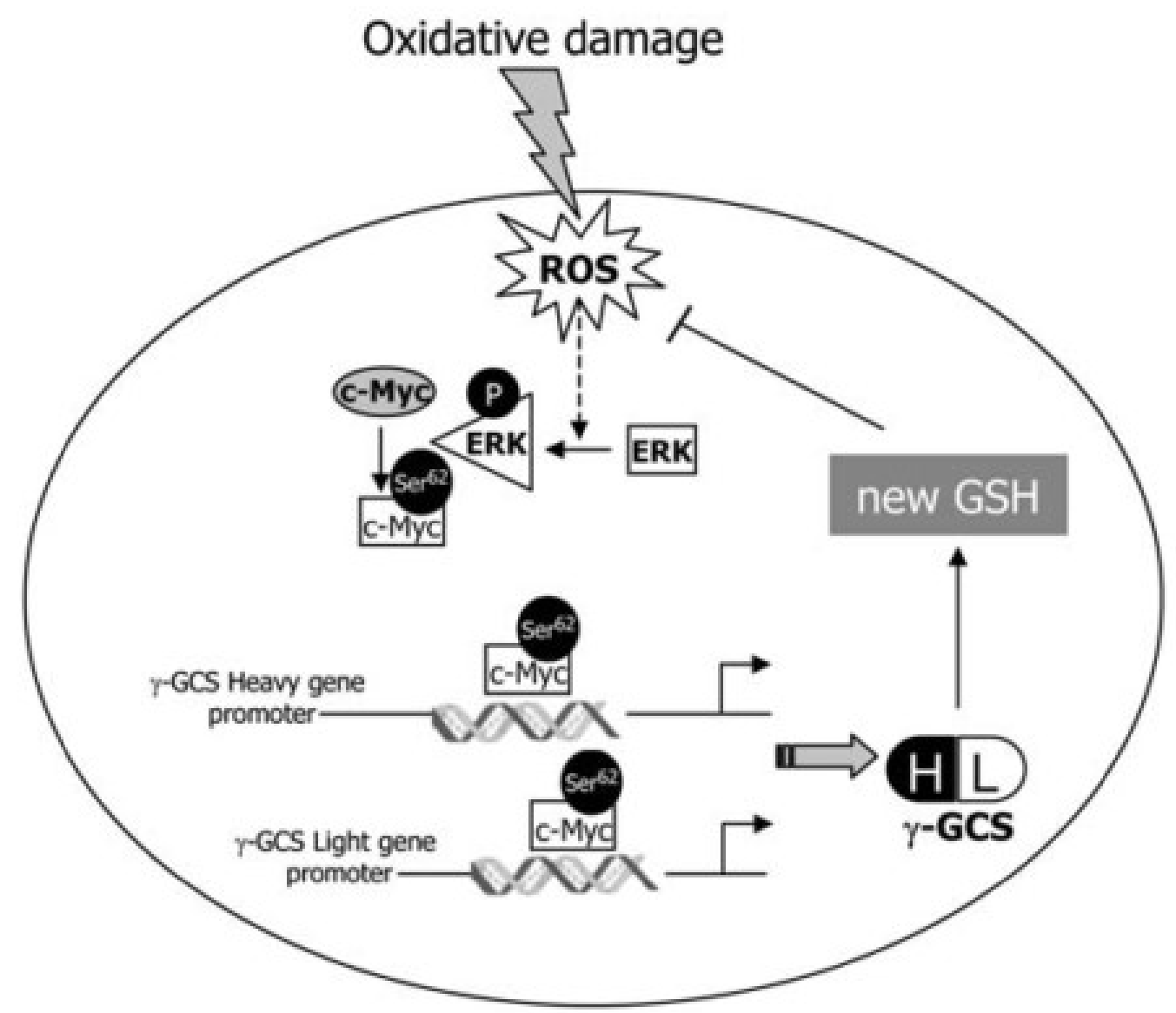

Figure 3.18 - A c-Myc-dependent survival pathway activated by oxidative stress within the cell as proposed by Benassi et al. (2006). 
Chapter 4 General Discussion

Went to school and I was very nervous No one knew me, no one knew me Hello teacher tell me what's my lesson Look right through me, look right through me 


\section{1 - Previous Studies}

Other researchers have explored the differential SMAD protein expression patterns in other animals that use environmental stress-triggered hypometabolism as a survival strategy. These include studies on the freeze tolerant wood frog (Rana sylvatica) and hibernating thirteen-lined ground squirrel (Ictidomys tridecemlineatus) (Aguilar, 2009; $\mathrm{Wu}, 2014)$. Their data demonstrated that SMAD regulation occurred in a tissue-specific and stress-specific response, providing further proof of the high complexity of TGF- $\beta$ signaling within the cells.

In studies of hibernating thirteen-lined ground squirrels experiencing a torpor-arousal cycle (Figure 4.1) total SMAD protein levels, specifically SMADs 2 and 3, remained constant in the skeletal muscle. In the liver, however, reduced expression levels of total SMAD2 and total SMAD3 were observed to occur selectively. Reductions included those to total SMAD2 in squirrels in late torpor and in interbout arousal, whereas total SMAD3 levels were significantly decreased as squirrels entered torpor. The phosphorylation state of SMAD2 in the liver and muscle of the squirrel remained constant throughout the torporarousal cycle, whereas SMAD3 showed differential phosphorylation in both tissues. In liver, p-SMAD3 increased by 1.4- and 1.6-fold in early torpor and late torpor, respectively. In skeletal muscle, meanwhile, only one significant increase was detected during entrance to torpor while over the rest of the cycle no changes occurred. SMAD4 protein, despite its limited role as a shuttle protein easing the translocation of R-SMADs into the nucleus, experienced the highest number of tissue significant differences through the torpor-arousal cycle in liver. In its hepatocytes, a $24 \%$ reduction occurred as the squirrels that were entering torpor ( $76 \%$ relative expression level to euthermic control), followed by an 
increase in early torpor and late torpor by 1.2-fold and 1.4-fold, respectively. Subsequently, during interbout arousal, SMAD4 expression was again suppressed by $56 \%$ compared to euthermic control in a cold room. Contrastingly, in skeletal muscle no differential SMAD4 regulation was observed. Although both SMADs 2 and 3 are differentially regulated in the liver, only SMAD3 appeared to affect gene expression in the squirrels through their torporarousal phase. CDK inhibitors $\mathrm{p} 21$ and $\mathrm{p} 15$ downstream of the TGF- $\beta$ signaling pathway were upregulated and helped to establish an anti-proliferation response during mammalian metabolic rate depression. Another study found that myostatin in the skeletal muscle of thirteen-lined ground squirrel could prevent atrophy when the signal was transduced by SMAD2 (threefold increase during early hibernation/torpor) and phospho-SMAD2 (fourfold rise during early torpor, five-fold increases during early arousal and interbout arousal) (Brooks et al., 2011). Other results of mention from the study include increased SMAD5 (1.5-fold during late hibernation) and greatly increased phospho-SMAD1/5 (eightfold during early hibernation, threefold during late hibernation, fourfold during early arousal) expression levels over the course of the torpor-arousal cycle of the hibernating squirrel.

These results showed that regulation of SMADs in skeletal muscle during the torporarousal cycle was absent despite increased differential transcription of p21CIP1 mRNA. Hepatocytes of the thirteen-lined ground squirrel showed differential expression of SMAD3 and an increased phosphorylated state of SMAD3 proteins during torpor (LT and EA). These were found to correlate with the increased expression levels of both $p 21 C I P 1$ and p15INK4b (Wu, 2014). The tissue-specific response to the torpor-arousal cycle observed was determined to be a consequence of physiology whereby myocytes are considered to be terminally differentiated and do not undergo active cell divisions whereas hepatocytes do proliferate (Montarras et al., 1989; Loyer et al., 2012). 
Wood frogs are freeze tolerant and must endure cycles of whole body freeze/thaw over the winter that can include oxidative stress. The metabolic response of SMAD transcription factors in the wood frog tissues typically rose as a result of freeze/thaw. Skeletal muscle of the frog showed the strongest activation of SMADs with p-SMAD1 (serine 465) increased by 1.4 -fold during $24 \mathrm{~h}$ freezing and 1.6 -fold during $8 \mathrm{~h}$ thaw. SMAD2 and p-SMAD2 (serines 465 and 467 ) rose to 1.6 and 1.8 -fold above control values during $24 \mathrm{~h}$ freezing and showed 1.6 and 2.4-fold increases after $8 \mathrm{~h}$ thaw, respectively. Total SMAD3 levels remained at control levels while freezing, but increased by $30 \%$ during $8 \mathrm{~h}$ thaw. The phosphorylated form, p-SMAD3 (serine 425 ) increased by $34 \%$ and $45 \%$ in freezing and thaw, respectively. No change in SMAD4 levels occurred in response to freeze/thaw even though it is typically closely linked with R-SMAD regulation.

In the same way that SMAD3 was implicated in triggering a cellular response to torpor in the hibernating squirrel, it was also found to play a major role in the gene response of the freezing frog. It displayed the strongest and most consistent response throughout the tissues studied (brain, muscle, liver, kidney, and heart). These findings suggested SMAD3 enhances the survival of the organism during periods of freezing and thawing by acting upon the genes SERPINE1 and TSC22D3 and mediating their transcriptional activation (Aguilar, 2009).

\section{2 - Anoxic Effects on SMAD Protein Expression and Cellular Processes}

In Chapter 2, the effects of anoxia on SMAD protein expression and various proteins downstream of the signaling pathway were explored. I used the red-eared slider (Trachemys scripta elegans) as the model organism as many studies have documented its ability, as well as that of other freshwater turtles (e.g. Chrysemys picta bellii), to survive 
long-term anoxia in cold water (Ultsch, 2006; Jackson, 2002). In this study, I showed that SMADs are regulated in a tissue-specific, animal-specific, and most importantly stressspecific way.

\subsection{1 - Anoxia in the Liver}

The data for liver provided evidence that was consistent with cell cycle arrest in this organ under anoxia (Biggar, 2013). Substantial decreases were observed for total R-SMAD (between $25 \%$ and $40 \%$ of control values) and, more importantly, their phosphorylated forms (p-SMAD3 expression was reduced to $30 \%$ of control) in response to long-term anoxia. Hepatocytes could have potentially transitioned into an angiostatic state where SMAD1 expression was reduced to about $60 \%$ of normoxic levels after $5 \mathrm{~h}$ and $20 \mathrm{~h}$ anoxia, and p-SMAD $1 / 5$ expression decreased to about $30 \%$ of the control value (Pardali et al., 2010; Weis and Cheresh, 2011). And lastly, p-c-Myc levels increase greatly within the liver by 1.9 -fold in $5 \mathrm{~h}$ anoxia and 2.2 -fold in $20 \mathrm{~h}$ anoxia and could be associated with mounting an antioxidant response to protect this important organ under hypoxic/anoxic conditions, the liver being the primary storage organ for glycogen and the source of glucose that is exported as a fuel source for most other organs (Benassi et al., 2006).

\subsection{2 - Anoxia in White Skeletal Muscle}

SMADs 2 and 3 did not display patterns that conformed to cell cycle arrest suggesting a post-mitotic state of the tissue (Biggar, 2013). This was supported by the unchanged phospho-p21 levels observed in turtle white skeletal muscle. Angiogenic pathways appeared to be functionally reduced to save energy via the suppression of SMADs 1 and 5 as well as the reduced phosphorylation level of SMAD1/5 (González-Núñez et al., 2013; Pardali et al., 2010). The minimal amounts of ROS produced are dealt with by using specific pathways at specific levels of exposure (Hansen et al., 2006). FoxO1 proteins can 
help in counteracting the potential for chemical damage (through its transcriptional action on genes of antioxidant enzymes such as MnSOD and catalase) at 5h anoxia, and subsequently the more adaptable FoxO3 transcription factor is upregulated at $20 \mathrm{~h}$ anoxia (Krivoruchko and Storey, 2013). Oxidative injury did not seem to be a significant factor in this tissue because the full range of antioxidant responses were not activated in the anoxic episode either during $5 \mathrm{~h}$ or $20 \mathrm{~h}$ exposure, only the FoxO transcription factors (Willmore and Storey, 1997).

\subsection{3 - Anoxia in Red Skeletal Muscle}

Red skeletal muscle has a great capacity to vary its functional characteristics to meet the energy demands of the red-eared slider turtle during the highly unfavourable periods of anoxia that it must endure in the winter months. This plasticity can play a role in the turtle's survival as red muscle is known to comprise up to $50 \%$ of an animal's tissue mass and as a metabolic organ can have implications in fuel and energy homeostasis (Joanisse, 2004). The strong reduction in phospho-SMAD3 expression in red muscle to below $50 \%$ of control levels at $5 \mathrm{~h}$ anoxia and continued suppression at that level in $20 \mathrm{~h}$ anoxia could be an indicator that the muscle is poised towards atrophy under anoxic conditions. The concomitant drop in SMAD4 during 20h anoxia supported this observation as well as the depression in phospho-SMAD2 following 20h anoxic exposure. These suppressions were likely linked to the decrease in c-Myc, allowing inhibitors such as p21 and p27 the ability to induce cell cycle arrest at the $\mathrm{G}_{1}$ phase, and possibly inducing an atrophy response (Mei et al., 2015). Angiogenic processes at first tried to adapt to the oxygen deprivation elevating total SMAD2 expression, maintaining SMAD1 expression, and increasing SMAD5 expression threefold. However, once it is clear that no further oxygen would be available to the muscle tissues, they may have entered an angiostatic state, facilitated by a reduced 
SMAD1 expression, and an upregulation of total SMAD2 protein levels by 3.5 -fold as compared with CTSE (Itoh et al., 2012). Additionally, the tissue was potentially shielded from oxidative stress effects through the upregulation of phospho-c-Myc, which is largely responsible for the activation and expression of redox enzymes that protect the cells from ROS (Benassi et al., 2006).

\subsection{4 - Anoxia in Kidney}

In general, kidneys are highly oxygen-sensitive organs due to the energy demands of the many types of ATP-dependent transporters needed to selectively resorb ions and metabolites from the kidney filtrate. Low oxygen availability can lead to chronic kidney disease. Renal tissue showed strong responses by SMADs to low oxygen conditions imposed by $5 \mathrm{~h}$ anoxic submergence; total SMAD2 and phospho-SMAD2 (serine 465/467) levels increased by 2.5 -fold and 1.8-fold, respectively, whereas SMAD3 was restricted to a modest reduction to $80 \%$ of controls. SMAD2 upregulation began at the cytokine level with elevated protein expression of the TGF- $\beta$ precursor protein by 1.4 -fold. However, once the turtle transitioned into long term anoxia (20h), TGF- $\beta$ precursor protein expression was severely suppressed to a mere $3.1 \%$ of control levels, leading to a concomitant fall in phospho-SMAD2 and phospho-SMAD3 expression levels that dropped to $53 \%$ and $26 \%$ of normoxic values, respectively. The common R-SMAD partner, SMAD4, was also suppressed to $44 \%$ and $48 \%$ relative expression levels in $5 \mathrm{~h}$ and $20 \mathrm{~h}$ anoxia conditions. These SMAD protein regulations were done in combination with enhanced expression of both phospho-p21 and c-Myc; 1.5 and 2.9-fold rises under $5 \mathrm{~h}$ and 20h anoxia conditions, respectively, in the case of phospho-p21, and a 2.1-fold increase at 20h anoxia in the case of c-Myc (Greenway and Storey, 2000; Biggar, 2013). These responses may be indicative of a stimulation of angiogenesis (blood vessel growth) in 
response to mounting hypoxia over the initial $5 \mathrm{~h}$ of anoxic submergence. The rise in SMAD1 expression by 2.6 -fold in $5 \mathrm{~h}$ anoxia gives an indication of initial angiogenic proliferation and migration. This could be a compensation response to improve blood delivery to the kidney as hypoxia becomes more severe. However, after $20 \mathrm{~h}$ anoxia this response appears to be shut down (Pardali et al., 2010; van Meeteren and ten Dijke, 2012). Oxidative stress protection followed a similar trend to that seen in liver where phospho-cMyc was upregulated during $5 \mathrm{~h}$ and 20h anoxia (1.7-fold and 1.4-fold relative increases, respectively) (Blokhina, et al., 2003; Hansen et al., 2006). FoxO proteins were also observed to be activated in the antioxidant response (Krivoruchko and Storey, 2013).

\subsection{5 - Anoxia in Cardiac Muscle}

TGF- $\beta$ regulation in cardiomyocytes displayed very complex patterning, although with the aid of downstream transcription factor responses, one could begin to elucidate the functions of each differentially expressed R-SMAD. Following anoxic submergence, phospho-SMAD3 levels were reduced to $72 \%$ of controls within $5 \mathrm{~h}$ and further to just $36 \%$ of controls after $20 \mathrm{~h}$. This could have indicated cell cycle arrest was occurring only after long-term oxygen deficiency (Goumans et al., 2003; Pardali et al., 2005). In 5h anoxia, total SMAD3 protein levels increased 2.2-fold providing evidence of a hypertrophic response of cardiomyocytes experiencing hypoxia or short-term anoxia (Akazawa and Komuro, 2003). TGF- $\beta$ precursor proteins, however, did not show the same suppression at $5 \mathrm{~h}$ anoxia and in fact protein expression levels were increased 1.7 -fold in $20 \mathrm{~h}$ anoxia. This result is not typical of the signaling pathway in the other tissues experiencing oxygen deprivation so a closer look was needed. SMAD2 expression was shown to be the benefactor of TGF- $\beta$ cytokine and subsequent ligand upregulation rising sharply to 1.7 fold of control expression in $5 \mathrm{~h}$ anoxia before dipping slightly to 1.5 -fold of controls in 
20h anoxia. However, SMAD2 phosphorylation was not differentially regulated by anoxic stress suggesting instead a role in angiogenesis. Total SMAD1 was initially lowered to $70 \%$ expression level at $5 \mathrm{~h}$ anoxia but then rose sharply to a 1.5 -fold over controls within cardiomyocytes at $20 \mathrm{~h}$ anoxia seemingly providing the argument for an angiogenic state (Masaki et al., 2005). However, this was amended by SMAD5 expression showing a rapid latent reduction to $32 \%$ of control expression levels and its phosphorylated analog (phospho-SMAD1/5) being significantly suppressed accordingly (reduction to 64\% relative expression). SMAD1 proteins could, therefore, be involved in inducing a homeostatic effect by helping to maintain cardiomyocyte vasculature during long-term anoxia although an overall angiostatic state is enforced by phospho-SMAD5 (Brown et al., 2004; Greenway and Storey, 2000). Activation of defense systems against ROS did not appear to occur until late into anoxic conditions. FoxO and ATF proteins work in a synchronized fashion to prompt an antioxidant response to protect the heart from oxidative injury (Akazawa and Komuro, 2003; Krivoruchko and Storey, 2012; Massagué, 2012; Kang et al., 2003). This response occurs in turn with cell cycle arrest suggesting that autophagy might play a role in preventing wasteful ATP consumption and limiting ROS production at the mitochondria (Glick et al., 2010).

These tissue-specific sets of regulation of the TGF- $\beta$ pathway truly seem to be adapted perfectly in the turtle to allow a balance between survival, low energy consumption, and minimal oxidative injury.

\section{3 - Dehydration Effects on SMAD Protein Expression and Cellular Processes}

In Chapter 3 dehydration effects were explored in the context of TGF- $\beta$ signaling and the genes that were affected downstream. Liver of the frog, $X$. laevis, showed the least 
number of significant changes in response to whole body dehydration, and those changes were modest. The data showed that SMAD expression in the liver was not altered much by dehydration. Evidence of cell cycle suppression was supplied from the observed decrease in SMAD3 expression (reduced to $60 \%$ of CXL), however the downstream proteins played major roles in maintaining that state (Siegel and Massagué, 2003; Pardali et al., 2005; Siegel and Massagué, 2003; Luu, 2013). c-Myc protein expression was halved during MD and maintained at that level during $\mathrm{HD}$, thus potentially allowing $\mathrm{CDK}$ inhibitors to be activated and promote the arrest of cell proliferation in the tissue (Hirschhorn et al., 2012). c-Fos expression further supports these arguments with 1.6- and 1.7- fold increases during dehydration causing an enhancing effect on the DNA-binding abilities of SMAD transcription factors (Shaulian and Karin, 2001; Bossis et al., 2006). Phosphorylation of cMyc (Thr 58, Ser 62) was hugely increased in hepatocytes (5.1-fold increase in MD and 8fold in HD) and could play a role in the antioxidant response of the cells. The intensity of this response indicates that during dehydration the frog could be subjected to either a large ROS challenge or that the frog is potentially more sensitive to ROS and must act very quickly to counteract their production to prevent unsustainable amounts of damage that could be deleterious to its cells (Benassi et al., 2006; França et al., 2007).

Muscles showed a stronger influence on its SMAD pathway regulation with downregulation of all R-SMAD isoforms, except SMAD5 in response to dehydration. Total SMAD2 showed the most profound difference with expression levels falling to $47 \%$ of CXL values. Again the downstream transcription factors were more acutely affected by dehydration with c-Fos expression rising by about a three-fold in muscle under high dehydration conditions, and phospho-c-Myc levels rising 2.5 fold. These data provide 
evidence that could indicate that the muscle to a lesser extent than liver experiences ROS stemming from dehydration and has induced cell cycle arrest in response (Blokhina et al., 2003; Biroccio et al., 2004; Hansen et al., 2006). The respective activation and deactivation of these processes ensure successful metabolic rate depression and the organism's survival of the extreme environmental conditions (Tanos et al., 2005; Monje et al., 2003; Malik and Storey; 2009, 2011).

\section{4 - Physiological Differences in TGF- $\beta$ Signaling}

The present studies of the anoxia-tolerant turtle $T$. scripta elegans and the dehydrating frog $X$. laevis add new information to our knowledge of the role of SMAD signaling in aiding cells and organisms to deal with environmental stress. Wood frogs, $R$. sylvatica, globally increased SMAD expression in its muscular tissues by between 1.5- and twofold in response to freezing as well as during recovery from subzero temperatures, whereas the thirteen-lined ground squirrel only increased phospho-SMAD3 expression during entrance into torpor/hibernation (no other SMADs showed differential expression over the torporarousal cycle) (Wu, 2014; Aguilar, 2009). The anuran (X. laevis) displayed a SMAD profile that was completely different and downregulated across the R-SMADs similar to the response of the white skeletal muscles in T. scripta elegans response to anoxic stress. The red skeletal muscle of the turtle had a signaling profile that shared more common elements with the wood frog, particularly total SMAD2 and phospho-SMAD2 upregulation in response to stress. Interestingly, active SMAD1 protein phosphorylated at serines 463/465 in wood frog had the same pattern and might also play a similar role as SMAD5 in the freshwater turtle. 
In hepatic tissues, the wood frog experienced few changes with phospho-SMAD3 being upregulated twofold and 2.5-fold during $24 \mathrm{~h}$ freezing and $8 \mathrm{~h}$ thaw, respectively, as compared with controls; SMAD2 expression was decreased at 8h thaw, and SMAD4 was suppressed by about half at $24 \mathrm{~h}$ freezing (Aguilar, 2009). None of these results aligned with the trends observed with the African clawed frog and the red-eared slider only matches the stress-induced SMAD4 downregulation and SMAD2 protein suppression, however, in turtle the origin of the reduction stems from anoxia and in the wood frog it occurs during the $8 \mathrm{~h}$ thaw. The hibernating squirrel profile lined up much better with $X$. laevis in this study. The squirrel showed many significant changes throughout the hibernation cycle, but the most important were the strong downregulation of total SMAD2 expression at late torpor and interbout arousal (25\% and 10\% expression levels relative to euthermic controls, respectively), upregulations of 1.4 and 1.6-fold of phospho-SMAD3 in early and late torpor, respectively, a strong suppression of total SMAD3 to $30 \%$ of controls, and the upregulation of SMAD4 at early and late torpor (1.2- and 1.4-fold, respectively) followed by the decrease to $60 \%$ relative expression levels at interbout arousal. X. laevis liver showed some of these effects although in a much more mild form such as total SMAD3 being suppressed to about $70 \%$ whereas the squirrel dropped the relative protein level down lower to $30 \%$. The anoxia-tolerant turtle showed similarities with the squirrel profile on two fronts: both total levels of SMAD2 and 3 were suppressed during stress conditions, and to about the same relative extent (70-80\% suppression). This similarity could stem from the need to highly regulate their main metabolic organ (liver) during their respective inactive periods. 
The SMAD expression patterns were not studied in the kidney or heart of hibernating squirrels nor in dehydration-tolerant frogs but only in wood frogs and red-eared slider turtles. The response to freezing stress by kidney in wood frogs included upregulation of phospho-SMAD1 expression by 2.2-fold after $24 \mathrm{~h}$ freezing along with the suppressions of total SMAD3 and p-SMAD3 to an 80\% expression level. After 8h thaw, SMAD2 and pSMAD2 expressions doubled whereas p-SMAD3 was maintained at an 80\% expression rate, and SMAD4 experienced was reduced by half. All of these changes were seen in the freshwater turtle, however total SMAD4, total SMAD2 and phospho-SMAD2 increases were not during seen during the recovery phase. Similar conditions probably develop within the kidneys of both animals in that both experience hypoxia (one localized, one global) and might require enhanced antioxidant defenses.

In the cardiac muscle tissues, it was observed that phospho-SMAD1 levels were halved during $24 \mathrm{~h}$ freezing, total and phosphorylated SMAD2 were reduced by between $50 \%$ and $60 \%$ in $24 \mathrm{~h}$ freezing and $8 \mathrm{~h}$ thaw, while phospho-SMAD3 surged to twofold and 1.5 -fold differences relative to control during $24 \mathrm{~h}$ freezing and $8 \mathrm{~h}$ thaw, respectively. None of these changes matched those found in the turtle heart experiencing anoxia.

\section{5 - Future Studies}

The regulation of SMADs within cardiac muscle and renal tissues has proven to be interesting as they play a major role in anoxia survival in the red-eared slider, and are proposed to play an equally important role in frog tolerance of dehydration. A deeper analysis into angiostatic and angiogenic proteins would allow more of an understanding as to how these pathways are regulated in non-human organisms. 
PCR analysis of gene transcript levels has been shown to be a poor determinant of SMAD protein levels in Rana sylvatica (wood frog) as transcripts increased, whereas protein levels do not change. This effect is presumed to be an anticipatory response, as organisms will recommence cell cycle, homeostatic, and anti-apoptotic pathways upon recovery from stress and will quickly transcribe the mRNA found in cytoplasmic stress granules into SMAD proteins (Aguilar, 2009). However, an analysis of the expression of genes that are downstream of the SMAD signaling pathway could make valuable additions to the understanding of the SMAD system and support the conclusions found in this thesis. For example, the $t s c 22 d 3$ gene (coding TSC22D3 protein) contains a SMAD binding element in its promoter region, is known to be expressed in response to osmotic stress, and has been shown to be upregulated in the wood frog in response to freezing. Analysis of the responses by this gene to anoxia in turtles or dehydration in $X$. laevis would be important. Another gene worth noting for future studies is serpine1, coding for the SERPINE1 protein. SERPINE1 determines the fate of many aspects of angiogenesis including the formation and maintenance of blood clots at the site of injury, be that a vessel or an organ.

Smurf2 ubiquitin ligase has been shown to tag SMAD proteins in the linker arm region to trigger their degradation by the proteasome. At high concentrations, Smurf2 has also been reported to suppress SMAD2 expression, but has shown a minimal effect on SMAD3 protein levels of $R$. sylvatica (Zhang et al., 2001). Analysis of Smurf2 protein expression may provide a deeper understanding of the signal transduction happening at the cellular level as SMAD turnover can terminate the induction of various mechanisms. SARA, a protein which facilitates the movement of SMAD complexes from the cell surface receptor 
through the cytoplasm, plays a part in the fate of SMAD proteins post-signaling and should be a target for further research.

Differential phosphorylation of R-SMADs in their linker arms by CDK8/9, as well as other protein kinases, may prove to be another route of interest for further research. These phosphorylation sites found outside either MH domain can inactivate SMADs and signal their degradation by proteasomes (mediated by GSK3 which additionally phosphorylates R-SMADs to be recognized by E3 ubiquitin ligases and proteasomes) to allow the unhindered operation of the cell cycle (Massagué, 2012).

Another avenue for future work would be DNA-binding studies that could be done to determine if phospho-SMADs effectively bind their targets within the nucleus under anoxic or dehydrated conditions. This study would be done using ELISA methods. The association of SMAD with many other proteins both within the same pathway and outside of it makes it imperative to determine what SMADs are bound to within cells under stress versus control situations, as well as the ratios in which these complexes appear. Such a study would be done using the method of co-immunoprecipitation. Another study could use a simple immunoprecipitation to isolate SMAD proteins and allow for probing of stressspecific posttranslational modifications to SMADs such as differential methylation or acetylation using pan-methyl lysine/arginine and pan-acetyl antibodies. Proteomic analysis could also be done with a purified sample to search for additional potential posttranslational modification sites unique to the organism. 


\section{6 - Concluding Remarks}

This study has shown the capability of the TGF- $\beta$ signaling to invoke a stress-induced response proportional and adapted to extreme conditions faced by different organisms. The pathway uses a multipronged approach to provide a flexible and effective solution to the myriad of stresses that a cell may encounter. Direct stimulation via cytokines are the first sign to SMAD proteins that trigger their phosphorylation that could, in turn, either steer them towards the nucleus to transduce their signals or remain in the cytoplasm in wait of more amenable conditions. These very complex signal profiles are what allow this pathway to be used across organisms and varying environmental conditions as a tool for survival. Processes that have been shown to be potentially differentially managed via SMADs are the cell cycle, the antioxidant response (via redox enzymatic activity), angiogenesis, and autophagy. All of these processes require regulation by cells in order to allow the organism to fully realize metabolic rate depression and stress-specific adaptive responses. It can be said that the SMAD pathway is a "Jack of all trades" in how widespread its effects are in the cell, and how tissue-specific it has been shown to be. Whether it is the African clawed frog's need to retain water and minimize fuel/energy use under dehydration/estivation conditions, or the red-eared slider turtle need to endure oxygen depletion while submerged under water, the TGF- $\beta$ pathway and its signal effectors are quickly recruited to mount a counterattack to conditions that could otherwise become lethal. 


\section{7 - General Discussion Figures}

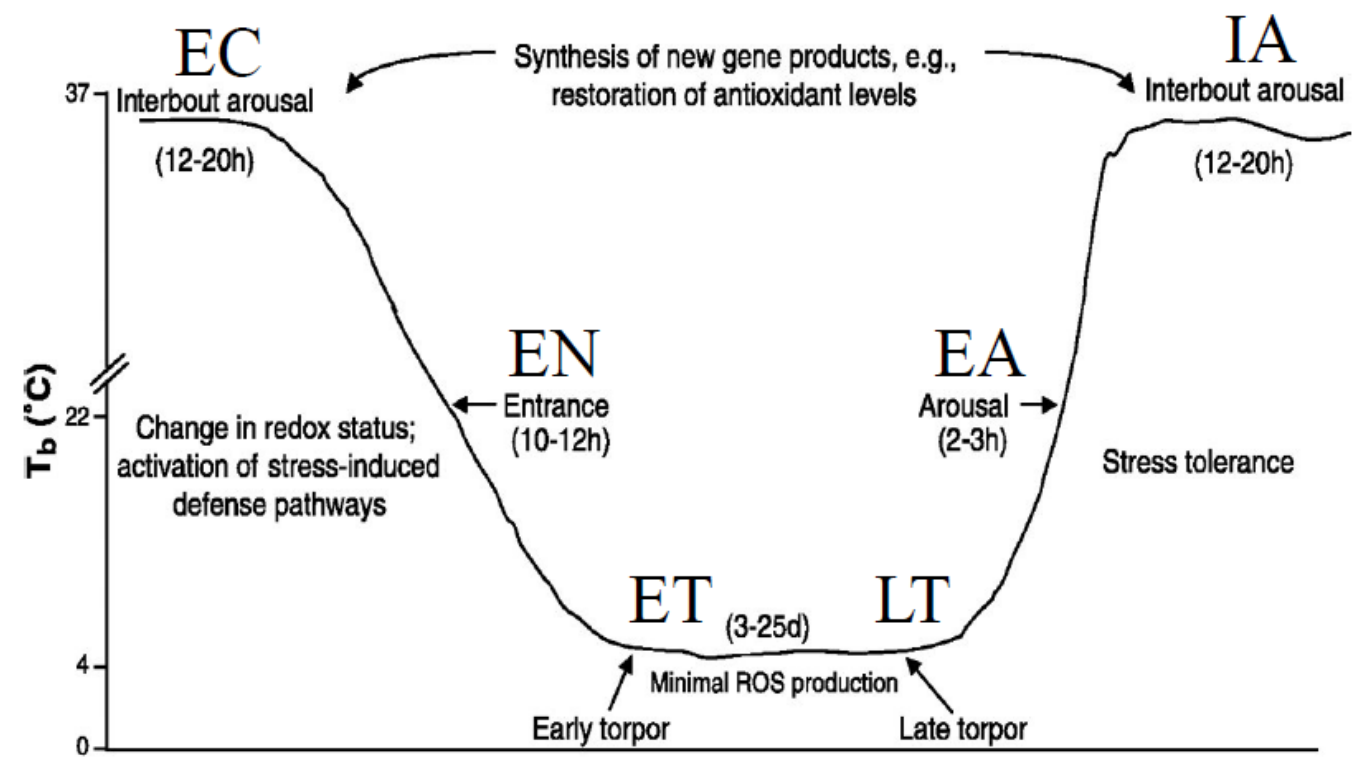

Time

Figure 4.1 - Stages of torpor-arousal cycle. 


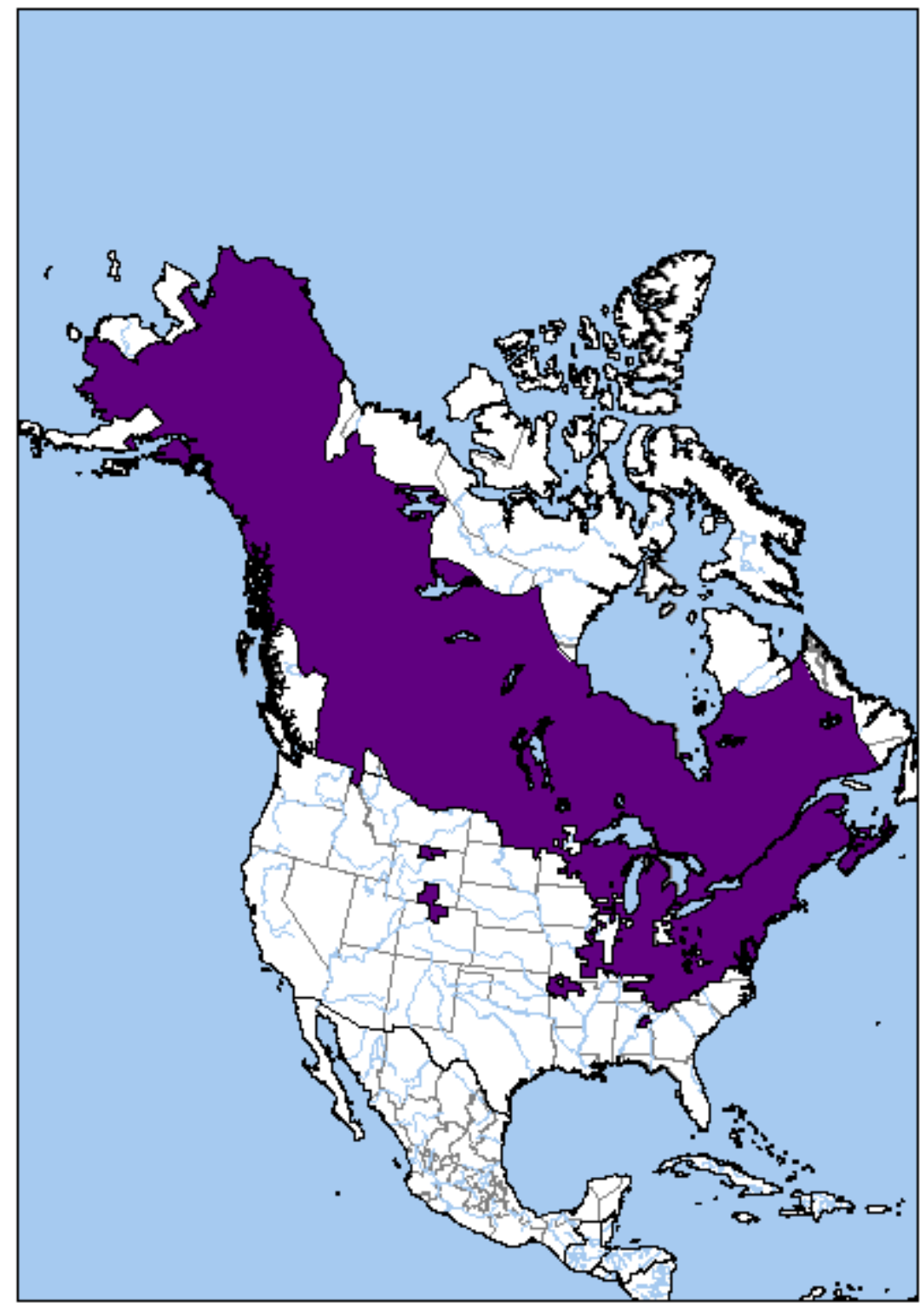

Figure 4.2 - The distribution of wood frog (Rana sylvatica) in the western hemisphere.

Figure from IUCN, Conservation International, NatureServe and collaborators (2004). 


\section{References}

1. Aguilar, O. A. Regulation of the MEF-2 and the SMAD Family of Transcription Factors in the Freeze Tolerant Wood Frog, Rana Sylvatica. (Carleton University, 2009).

2. Akazawa, H. \& Komuro, I. Roles of cardiac transcription factors in cardiac hypertrophy. Circ. Res. 92, 1079-1088 (2003).

3. Akman, H. O. et al. Response to hypoxia involves transforming growth factor-2 and Smad proteins in human endothelial cells. Blood 98, 3324-3332 (2001).

4. Alani, R. M., Young, A. Z. \& Shifflett, C. B. Id1 regulation of cellular senescence through transcriptional repression of p16/Ink4a. PNAS 98, 7812-7816 (2001).

5. Alarcón, C. et al. CDK8/9 drive Smad transcriptional action, turnover and YAP interactions in BMP and TGF $\beta$ pathways. Cell 139, 757-769 (2009).

6. Allen, D. L. \& Unterman, T. G. Regulation of myostatin expression and myoblast differentiation by FoxO and SMAD transcription factors. Am. J. Physiol. Cell Physiol. 292, C188-C199 (2007).

7. Alliston, T., Piek, E. \& Derynck, R. in The TGF- $\beta$ Family (eds. Derynck, R. \& Miyazono, K.) 667-724 (Cold Spring Harbour Laboratory Press, 2008).

8. Attisano, L. \& Wrana, J. L. Mads and Smads in TGF- $\beta$ signalling. Curr. Opin. Cell Biol. 10, 188-194 (1998).

9. Attisano, L. \& Wrana, J. L. Signal transduction by the TGF- $\beta$ superfamily. Science 296, 1646-1647 (2002).

10. Attisano, L. \& Wrana, J. L. Smads as transcriptional co-modulators. Curr. Opin. Cell Biol. 12, 235-243 (2000). 
11. Balinsky, B. Y. J. B. \& Baldwin, E. The mode of excretion of ammonia and urea in Xenopus laevis. J. Exp. Biol. 38, 695-705 (1961).

12. Balinsky, J. B., Choritz, E. L., Coe, C. G. \& van der Schans, G. S. Urea cycle enzymes and urea excretion during the development and metamorphosis of Xenopus laevis. Comp. Biochem. Physiol. 22, 53-57 (1967).

13. Balinsky, J. B., Cragg, M. M. \& Baldwin, E. The adaptation of amphibian waste nitrogen excretion to dehydration. Comp. Biochem. Physiol. 3, 236-244 (1961).

14. Balinsky, J. B., Choritz, E. L., Coe, C. G. L. \& van der Schans, G. S. Amino acid metabolism and urea synthesis in naturally aestivating Xenopus laevis. Comp. Biochem. Physiol. 22, 59-68 (1967).

15. Barnhart, M. C. Gas permeability of the epiphragm of a terrestrial snail, Otala lactea. Physiol. Zool. 56, 436-444 (1983).

16. Benassi, B. et al. c-Myc phosphorylation is required for cellular response to oxidative stress. Mol. Cell 21, 509-519 (2006).

17. Biggar, K. K. Cell cycle regulation by post-translational and post-transcriptional mechanisms in an anaerobic extremist - The anoxic tolerant turtle, Trachemys scripta elegans. (Carleton University, 2013).

18. Biggar, K. K. \& Storey, K. B. Evidence for cell cycle suppression and microRNA regulation of cyclin D1 during anoxia exposure in turtles. Cell Cycle 11, 1705-1713 (2012).

19. Biggar, K. K. \& Storey, K. B. Perspectives in cell cycle regulation: lessons from an anoxic vertebrate. Curr. Genomics 10, 573-584 (2009).

20. Biroccio, A. et al. Glutathione influences c-Myc-induced apoptosis in M14 human melanoma cells. J. Biol. Chem. 277, 43763-43770 (2002). 
21. Biroccio, A., Benassi, B., Fiorentino, F. \& Zupi, G. Glutathione depletion induced by c-Myc downregulation triggers apoptosis on treatment with alkylating agents. Neoplasia 6, 195-206 (2004).

22. Blokhina, O. Antioxidants, oxidative damage and oxygen deprivation stress: a review. Ann. Bot. 91, 179-194 (2003).

23. Bossis, G. et al. Dimer activity by sumoylation down-regulation of c-Fos / c-Jun AP-1 dimer activity by sumoylation. Mol. Cell. Biol. 25, 6964-6979 (2005).

24. Brezis, M., Rosen, S., Spokes, K., Silva, P. \& Epstein, F. H. Transport-dependent anoxic cell injury in the isolated perfused rat kidney. Am. J. Pathol. 116, 327-341 (1984).

25. Brooks, N. E., Myburgh, K. H. \& Storey, K. B. Myostatin levels in skeletal muscle of hibernating ground squirrels. J. Exp. Biol. 214, 2522-2527 (2011).

26. Brown, C. O. et al. The cardiac determination factor, Nkx2-5, is activated by mutual cofactors GATA-4 and Smad1/4 via a novel upstream enhancer. J. Biol. Chem. 279, 10659-10669 (2004).

27. Brugarolas, J. et al. Regulation of mTOR function in response to hypoxia by REDD1 and the TSC1 / TSC2 tumor suppressor complex. Genes Dev. 18, 2893-2904 (2004).

28. Chen, J. \& Mandel, L. J. Role of water and electrolyte influxes in anoxic plasma membrane disruption. Am. J. Physiol. 273, C1341-C1348 (1997).

29. Cherkasov, V. et al. Systemic control of protein synthesis through sequestration of translation and ribosome biogenesis factors during severe heat stress. FEBS Lett. 589, 3654-3664 (2015).

30. Choy, L., Skillington, J. \& Derynck, R. Roles of autocrine TGF- $\beta$ receptor and Smad signaling in adipocyte differentiation. J. Cell Biol. 149, 667-681 (2000). 
31. Chuva de Sousa Lopes, S. M. et al. Connective tissue growth factor expression and Smad signaling during mouse heart development and myocardial infarction. Dev. Dyn. 231, 542-550 (2004).

32. Clark, V. M. \& Miller, A. T. Studies on anaerobic metabolism in the fresh-water turtle (Pseudemys scripta elegans). Comp. Biochem. Physiol. A. Comp. Physiol. 44, 55-62 (1973).

33. Cowan, K. J. Mitogen-activated protein kinases: new signaling pathways functioning in cellular responses to environmental stress. J. Exp. Biol. 206, 11071115 (2003).

34. Damsgaard, C., Storz, J. F., Hoffmann, F. G. \& Fago, A. Hemoglobin isoform differentiation and allosteric regulation of oxygen binding in the turtle, Trachemys scripta. Am. J. Physiol. Regul. Integr. Comp. Physiol. 305, R961-R967 (2013).

35. Dash, B. C. \& El-Deiry, W. S. Phosphorylation of $\mathrm{p} 21$ in G2/M promotes cyclin Bcdc2 kinase activity. Mol. Cell. Biol. 25, 3364-3387 (2005).

36. DeLaney, R. G., Lahiri, S., Hamilton, R. \& Fishman, P. Acid-base balance and plasma composition in the aestivating lungfish (Protopterus). Am. J. Physiol. 232, R10-R17 (1977).

37. Deneke, S. M. \& Fanburg, B. L. Regulation of cellular glutathione. Am. J. Physiol. 257, L163-L173 (1989).

38. Derynck, R. SMAD proteins and mammalian anatomy. Nature 393, 737-739 (1998).

39. Derynck, R. \& Feng, X.-H. TGF- $\beta$ receptor signaling. Biochim. Biophys. Acta 1333, F105-F150 (1997).

40. Derynck, R. \& Miyazono, K. in The TGF- $\beta$ Family (eds. Derynck, R. \& Miyazono, K.) 29-44 (Cold Spring Harbour Laboratory Press, 2008). doi:10.1086/596254 
41. Derynck, R., Zhang, Y. \& Feng, X. H. Smads: transcriptional activators of TGF- $\beta$ responses. Cell 95, 737-740 (1998).

42. Douglas, R. M., Xu, T. \& Haddad, G. G. Cell cycle progression and cell division are sensitive to hypoxia in Drosophila melanogaster embryos. Am. J. Physiol. Regul. Integr. Comp. Physiol. 280, R1555-R1563 (2001).

43. Dzieran, J. et al. Comparative analysis of TGF- $\beta /$ Smad signaling dependent cytostasis in human hepatocellular carcinoma cell lines. PLoS One 8, e72252 (2013).

44. Euler-Taimor, G. \& Heger, J. The complex pattern of SMAD signaling in the cardiovascular system. Cardiovasc. Res. 69, 15-25 (2006).

45. Fantin, A. et al. Tissue macrophages act as cellular chaperones for vascular anastomosis downstream of VEGF-mediated endothelial tip cell induction. Nature 465, 829-840 (2010).

46. Fei, T. et al. Genome-wide mapping of SMAD target genes reveals the role of BMP signaling in embryonic stem cell fate determination. Genome Res. 20, 36-44 (2010).

47. Feng, X.-H. \& Derynck, R. Specificity and versatility in TGF- $\beta$ signaling through Smads. Annu. Rev. Cell Dev. Biol. 21, 659-693 (2005).

48. Feng, X.-H., Liang, Y.-Y., Liang, M., Zhai, W. \& Lin, X. Direct interaction of cMyc with Smad2 and Smad3 to inhibit TGF- $\beta$-mediated induction of the CDK inhibitor p15Ink4B. Mol. Cell 9, 133-143 (2002).

49. Feng, X.-H., Lin, X. \& Derynck, R. Smad2, Smad3 and Smad4 cooperate with Sp1 to induce p15Ink4B transcription in response to TGF- $\beta$. EMBO J. 19, 5178-5193 (2000).

50. Feng, X.-H., Zhang, Y., Wu, R.-Y. \& Derynck, R. The tumor suppressor Smad4/DPC4 and transcriptional adaptor CBP/p300 are coactivators for Smad3 in TGF- $\beta$-induced transcriptional activation. Genes Dev. 12, 2153-2163 (1998). 
51. França, M. B., Panek, A. D. \& Eleutherio, E. C. Oxidative stress and its effects during dehydration. Comp. Biochem. Physiol. Part A Mol. Integr. Physiol. 146, 621631 (2007).

52. Franzén, P., Heldin, C. H. \& Miyazono, K. The GS domain of the transforming growth factor- $\beta$ type I receptor is important in signal transduction. Biochem. Biophys. Res. Commun. 207, 682-689 (1995).

53. Franzén, P. et al. Cloning of a TGF- $\beta$ type I receptor that forms a heteromeric complex with the TGF- $\beta$ type II receptor. Cell 75, 681-692 (1993).

54. Gatten, R. E. J. Activity metabolism of anuran amphibians: tolerance to dehydration. Physiol. Zool. 60, 576-585 (1987).

55. Ghesquière, B., Wong, B. W., Kuchnio, A. \& Carmeliet, P. Metabolism of stromal and immune cells in health and disease. Nature 511, 167-176 (2014).

56. Ghosh Choundhury, G. et al. Bone morphogenetic protein 2 inhibits platelet-derived growth factor- induced c-Fos gene transcription and DNA synthesis in mesangial cells. Involvement of mitogen-activated protein kinase. J. Biol. Chem. 274, 1089710902 (1999).

57. Glick, D., Barth, S. \& Macleod, K. F. Autophagy: cellular and molecular mechanisms. J. Pathol. 221, 3-12 (2010).

58. Goda, N. et al. Hypoxia-inducible factor $1 \alpha$ is essential for cell cycle arrest during hypoxia. Mol. Cell. Biol. 23, 359-369 (2003).

59. González-Núñez, M., Muñoz-Félix, J. M. \& López-Novoa, J. M. The ALK-1/Smad1 pathway in cardiovascular physiopathology. A new target for therapy? Biochim. Biophys. Acta 1832, 1492-1510 (2013).

60. Goumans, M. J. et al. Activin receptor-like kinase (ALK) 1 is an antagonistic mediator of lateral TGF $\beta /$ ALK5 signaling. Mol. Cell 12, 817-828 (2003). 
61. Greenway, S. C. \& Storey, K. B. Mitogen-activated protein kinases and anoxia tolerance in turtles. J. Exp. Zool. 287, 477-484 (2000).

62. Grundy, J. E. \& Storey, K. B. Urea and salt effects on enzymes from estivating and non-estivating amphibians. Mol. Cell. Biochem. 131, 9-17 (1994).

63. Halazonetis, T. D., Georgopoulos, K., Greenberg, M. E. \& Leder, P. c-Jun dimerizes with itself and with c-Fos, forming complexes of different DNA binding affinities. Cell 55, 917-924 (1988).

64. Hansen, J. M., Go, Y.-M. \& Jones, D. P. Nuclear and mitochondrial compartmentation of oxidative stress and redox signaling. Annu. Rev. Pharmacol. Toxicol. 46, 215-234 (2006).

65. Heldin, C. H., Miyazono, K. \& ten Dijke, P. TGF- $\beta$ signalling from cell membrane to nucleus through SMAD proteins. Nature 390, 465-471 (1997).

66. Hillman, S. S. The roles of oxygen delivery and electrolyte levels in the dehydrational death of Xenopus laevis. J. Comp. Physiol. B Biochem. Syst. Environ. Physiol. B 128, 169-175 (1978).

67. Hillman, S. S. Physiological correlates of differential dehydration tolerance in anuran amphibians. Copeia 1980, 125-129 (1980).

68. Hirschhorn, T., Barizilay, L., Smorodinsky, N. I. \& Ehrlich, M. Differential regulation of Smad3 and of the type II transforming growth factor- $\beta$ receptor in mitosis: implications for signaling. PLoS One 7, e43459 (2012).

69. Hochachka, P. W., Buck, L. T., Doll, C. J. \& Land, S. C. Unifying theory of hypoxia tolerance: molecular/metabolic defense and rescue mechanisms for surviving oxygen lack. PNAS 93, 9493-9498 (1996). 
70. Hochachka, P. W. \& Lutz, P. L. Mechanism, origin, and evolution of anoxia tolerance in animals. Comp. Biochem. Physiol. Part B Biochem. Mol. Biol. 130, 435-459 (2001).

71. Hoekstra, F., Golovina, E. \& Buitink, J. Mechanisms of plant desiccation tolerance. Trends Plant Sci. 6, 431-438 (2001).

72. Huang, X. R. et al. Smad3 mediates cardiac inflammation and fibrosis in angiotensin II-induced hypertensive cardiac remodeling. Hypertension 55, 1165-1171 (2010).

73. Huse, M. et al. The TGF- $\beta$ receptor activation process. Mol. Cell 8, 671-682 (2001).

74. Hybertson, B. M., Gao, B., Bose, S. K. \& McCord, J. M. Oxidative stress in health and disease: the therapeutic potential of Nrf2 activation. Mol. Aspects Med. 32, 234 246 (2011).

75. Ijichi, H. et al. Smad4-independent regulation of p21/WAF1 by transforming growth factor- $\beta$. Oncogene 23, 1043-1051 (2004).

76. Ireland, M. P. Studies on the adaptation of Xenopus laevis to hyperosmotic media. Comp. Biochem. Physiol. A. Comp. Physiol. 46, 469-476 (1973).

77. Itoh, F. et al. The FYVE domain in Smad anchor for receptor activation (SARA) is sufficient for localization of SARA in early endosomes and regulates TGF- $\beta / \mathrm{Smad}$ signalling. Genes to Cells 7, 321-331 (2002).

78. Itoh, F. et al. Smad2/Smad3 in endothelium is indispensable for vascular stability via S1PR1 and N-cadherin expressions. Blood 119, 5320-5328 (2012).

79. Itoh, S., Itoh, F., Goumans, M. J. \& Ten Dijke, P. Signaling of transforming growth factor-beta family members through Smad proteins. Eur. J. Biochem. 267, 6954 6967 (2000). 
80. Jackson, D. C. Metabolic depression and oxygen depletion in the diving turtle. $J$. Appl. Physiol. 24, 503-509 (1968).

81. Jackson, D. C. Living without oxygen: lessons from the freshwater turtle. Comp. Biochem. Physiol. Part A Mol. Integr. Physiol. 125, 299-315 (2000).

82. Jackson, D. C. Hibernating without oxygen: physiological adaptations of the painted turtle. J. Physiol. 543, 731-737 (2002).

83. Jackson, D. C. \& Herbert, C. V. Temperature effects on the responses to prolonged submergence in the turtle Chrysemys picta bellii. II. metabolic rate, blood acid-base and ionic changes, and cardiovascular function in aerated and anoxic water. Physiol. Zool. 58, 670-681 (1985).

84. Jackson, D. C. et al. Comparative shell buffering properties correlate with anoxia tolerance in freshwater turtles. Am. J. Physiol. Regul. Integr. Comp. Physiol. 292, R1008-R1015 (2007).

85. Janssens, P. A. Urea production and transaminase activity in Xenopus laevis Daudin. Comp. Biochem. Physiol. 13, 217-224 (1964).

86. Joanisse, R. in Functional Metabolism: Regulation and Adaptation (ed. Storey, K. B.) 295-318 (John Wiley \& Sons, Inc., 2004).

87. Kamato, D. et al. Transforming growth factor $\beta$-mediated site-specific Smad linker region phosphorylation in vascular endothelial cells. J. Pharm. Pharmacol. 66, 1722-1733 (2014).

88. Kang, J. S., Liu, C. \& Derynck, R. New regulatory mechanisms of TGF- $\beta$ receptor function. Trends Cell Biol. 19, 385-394 (2009).

89. Kang, Y., Chen, C. R. \& Massagué, J. A self-enabling TGF- $\beta$ response coupled to stress signaling: Smad engages stress response factor ATF3 for Id 1 repression in epithelial cells. Mol. Cell 11, 915-926 (2003). 
90. Korchynskyi, O. \& Ten Dijke, P. Identification and functional characterization of distinct critically important bone morphogenetic protein-specific response elements in the Id1 promoter. J. Biol. Chem. 277, 4883-4891 (2002).

91. Kouzarides, T. \& Ziff, E. The role of the leucine zipper in the fos-jun interaction. Nature 336, 646-651 (1988).

92. Kretschmer, A. et al. Differential regulation of TGF- $\beta$ signaling through Smad2, Smad3 and Smad4. Oncogene 22, 6748-6763 (2003).

93. Kretzschmar, M. \& Massagué, J. SMADs: mediators and regulators of TGF- $\beta$ signaling I-III. Curr. Opin. Genet. Dev. 8, 103-111 (1998).

94. Krivoruchko, A. Turtle Anoxia - Biochemistry and Gene Regulation in an Anaerobic Extremist. (Carleton University, 2010).

95. Krivoruchko, A. \& Storey, K. B. Forever young: mechanisms of natural anoxia tolerance and potential links to longevity. Oxid. Med. Cell. Longev. 3, 186-198 (2010).

96. Krivoruchko, A. \& Storey, K. B. Anoxia-responsive regulation of the FoxO transcription factors in freshwater turtles, Trachemys scripta elegans. Biochim. Biophys. Acta 1830, 4990-4998 (2013).

97. Krivoruchko, A. \& Storey, K. B. Turtle anoxia tolerance: biochemistry and gene regulation. Biochim. Biophys. Acta - Gen. Subj. 1850, 1188-1196 (2015).

98. Krivoruchko, A. \& Storey, K. B. Activation of the unfolded protein response during anoxia exposure in the turtle Trachemys scripta elegans. Mol. Cell. Biochem. 374, 91-103 (2012).

99. Lando, D., Peet, D. J., Whelan, D. a, Gorman, J. J. \& Whitelaw, M. L. Asparagine hydroxylation of the HIF transactivation domain a hypoxic switch. Science 295, 858-861 (2002). 
100. Langley, B. Myostatin inhibits myoblast differentiation by down-regulating MyoD expression. J. Biol. Chem. 277, 49831-49840 (2002).

101. Lemons, J. M. S. et al. Quiescent fibroblasts exhibit high metabolic activity. PLoS Biol. 8, e1000514 (2010).

102. Lempradl, A., Pospisilik, J. A. \& Penninger, J. M. Exploring the emerging complexity in transcriptional regulation of energy homeostasis. Nat. Rev. Genet. 16, 665-681 (2015).

103. Li, W., Chen, F., Nagarajan, R. P., Liu, X. \& Chen, Y. Characterization of the DNAbinding property of Smad5. Biochem. Biophys. Res. Commun. 286, 1163-1169 (2001).

104. Liberati, N. T. et al. Smads bind directly to the Jun family of AP-1 transcription factors. PNAS 96, 4844-4849 (1999).

105. Liu, D., Black, B. L. \& Derynck, R. TGF- $\beta$ inhibits muscle differentiation through functional repression of myogenic transcription factors by Smad3. Genes Dev. 15, 2950-2966 (2001).

106. Liu, D., Kang, J. S. \& Derynck, R. TGF- $\beta$-activated Smad3 represses MEF2dependent transcription in myogenic differentiation. EMBO J. 23, 1557-1566 (2004).

107. Loveridge, J. P. \& Withers, P. C. Metabolism and water balance of active and cocooned African bullfrogs Pyxicephalus adspersus. Physiol. Zool. 54, 203-214 (1981).

108. Loyer, P., Corlu, A. \& Desdouets, C. Regulation of the hepatocyte cell cycle: signaling pathways and protein kinases. Int. J. Hepatol. 2012, ID 592354 (2012). 
109. Luu, B. E. D. Regulation of Protein Translation and Cell Cycle Processes by Reversible Protein Phosphorylation in Response to Dehydration in the African Clawed Frog. (Carleton University, 2013).

110. Macias, M. J., Martin-Malpartida, P. \& Massagué, J. Structural determinants of Smad function in TGF- $\beta$ signaling. Trends Biochem. Sci. 40, 296-308 (2015).

111. Macías-Silva, M. et al. MADR2 is a substrate of the TGF $\beta$ receptor and its phosphorylation is required for nuclear accumulation and signaling. Cell 87, 12151224 (1996).

112. Malik, A. I. Cellular adaptation to dehydration stress: Molecular adaptations for dehydration tolerance in the African clawed frog, Xenopus laevis. (Carleton University, 2009).

113. Malik, A. I. \& Storey, K. B. Activation of extracellular signal-regulated kinases during dehydration in the African clawed frog, Xenopus laevis. J. Exp. Biol. 212, 2595-2603 (2009).

114. Malik, A. I. \& Storey, K. B. Transcriptional regulation of antioxidant enzymes by FoxO1 under dehydration stress. Gene 485, 114-119 (2011).

115. Malik, A. I. \& Storey, K. B. Activation of antioxidant defense during dehydration stress in the African clawed frog. Gene 442, 99-107 (2009).

116. Masaki, M., Izumi, M. \& Oshima, Y. Smad1 protects cardiomyocytes from ischemia-reperfusion injury. Circulation 111, 2752-2759 (2005).

117. Massagué, J. TGF- $\beta$ signalling in context. Nat. Rev. Mol. Cell Biol. 13, 616-630 (2012).

118. Massagué, J. Transcriptional control by the TGF-beta/Smad signaling system. EMBO J. 19, 1745-1754 (2000). 
119. Massagué, J., Seoane, J. \& Wotton, D. Smad transcription factors. Genes Dev. 19, 2783-2810 (2005).

120. McClanahan, L. L., Ruibal, R. \& Shoemaker, V. H. Frogs and toads in deserts. Sci. Am. 270, 82-88 (1994).

121. McClanahan, L. Adaptations of the spadefoot toad Scaphiopus couchii, to desert environments. Comp. Biochem. Physiol. 20, 73-99 (1967).

122. Mei, Z. et al. FBXO32 targets c-Myc for proteasomal degradation and inhibits cMyc activity. J. Biol. Chem. 290, 16202-16214 (2015).

123. Mizushima, N. Autophagy: process and function. Genes Dev. 21, 2861-2873 (2007).

124. Monje, P., Marinissen, M. J. \& Gutkind, J. S. Phosphorylation of the carboxylterminal transactivation domain of c-Fos by extracellular signal-regulated kinase mediates the transcriptional activation of AP-1 and cellular transformation induced by platelet-derived growth factor. Mol. Cell. Biol. 23, 7030-7043 (2003).

125. Montarras, D., Pinset, C., Kahn, A. \& Gros, F. Expression of MyoD1 coincides with terminal differentiation in determined but inducible muscle cells. EMBO J. 8, 22032207 (1989).

126. Moustakas, A. \& Kardassis, D. Regulation of the human $\mathrm{p} 21 / \mathrm{WAF} 1 / \mathrm{Cip} 1$ promoter in hepatic cells by functional interactions between $\mathrm{Sp} 1$ and Smad family members. PNAS 95, 6733-6738 (1998).

127. Nicolás, F. J. \& Hill, C. S. Attenuation of the TGF- $\beta$-Smad signaling pathway in pancreatic tumor cells confers resistance to TGF- $\beta$-induced growth arrest. Oncogene 22, 3698-3711 (2003).

128. Orrenius, S., Zhivotovsky, B. \& Nicotera, P. Regulation of cell death: the calciumapoptosis link. Nat. Rev. Mol. cell Biol. 4, 552-565 (2003). 
129. Oshitari, T. et al. The role of c-Fos in cell death and regeneration of retinal ganglion cells. Investig. Ophthalmol. Vis. Sci. 43, 2442-2449 (2002).

130. Page, A., Kirkpatrick, W. \& Massam, M. Risk Assessments for Exotic Reptiles and Amphibians Introduced into Australia - African Clawed Frog (Xenopus laevis; Daudin, 1802). (2008). at $<\mathrm{http} / / / \mathrm{www}$.issg.org/>

131. Pardali, E., Goumans, M.-J. \& ten Dijke, P. Signaling by members of the TGF- $\beta$ family in vascular morphogenesis and disease. Trends Cell Biol. 20, 556-567 (2010).

132. Pardali, K., Kowanetz, M., Heldin, C. H. \& Moustakas, A. Smad pathway-specific transcriptional regulation of the cell cycle inhibitor p21WAF1/Cip1. J. Cell. Physiol. 204, 260-272 (2005).

133. Pardoux, C. \& Derynck, R. JNK regulates expression and autocrine signaling of TGF-ß1. Mol. Cell 15, 170-171 (2004).

134. Pereira, E. D. J., Panek, A. D. \& Eleutherio, E. C. A. Protection against oxidation during dehydration of yeast. Cell Stress Chaperones 8, 120-124 (2003).

135. Qing, J., Zhang, Y. \& Derynck, R. Structural and functional characterization of the transforming growth factor- $\beta$-induced Smad3/c-Jun transcriptional cooperativity. $J$. Biol. Chem. 275, 38802-38812 (2000).

136. Reese, S. A., Jackson, D. C. \& Ultsch, G. R. Hibernation in freshwater turtles: softshell turtles (Apalone spinifera) are the most intolerant of anoxia among North American species. J. Comp. Physiol. B Biochem. Syst. Environ. Physiol. 173, 263268 (2003).

137. Romspert, A. P. Osmoregulation of the African clawed frog. Xenopus laevis, in hypersaline media. Comp. Biochem. Physiol. Part A Physiol. 54, 207-210 (1976). 
138. Sanchiz, B. \& Rocek, Z. in The Biology of Xenopus (eds. Tinsley, R. C. \& Kobel, H. R.) 317-328 (Zoological Society of London, 1996).

139. Sartori, R. et al. Smad2 and 3 transcription factors control muscle mass in adulthood. Am. J. Physiol. Cell Physiol. 296, C1248-C1257 (2009).

140. Schipani, E. et al. Hypoxia in cartilage: HIF-1 $\alpha$ is essential for chondrocyte growth arrest and survival. Genes Dev. 15, 2865-2876 (2001).

141. Schmierer, B. \& Hill, C. S. TGF- $\beta$-SMAD signal transduction: molecular specificity and functional flexibility. Nat. Rev. Mol. cell Biol. 8, 970-982 (2007).

142. Schmierer, B., Tournier, A. L., Bates, P. \& Hill, C. S. Mathematical modeling identifies Smad nucleocytoplasmic shuttling as a dynamic signal-interpreting system. PNAS 105, 6608-6613 (2008).

143. Secor, S. M. Physiological responses to feeding, fasting and estivation for anurans. J. Exp. Biol. 208, 2595-2608 (2005).

144. Seiter, P., Schultheiss, H. \& Hanke, W. Osmotic stress and excretion of ammonia and urea in Xenopus laevis. Comp. Biochem. Physiol. Part A Physiol. 61, 571-576 (1978).

145. Shaulian, E. \& Karin, M. AP-1 in cell proliferation and survival. Oncogene 20, 2390-2400 (2001).

146. Shi, Y. \& Massagué, J. Mechanisms of TGF- $\beta$ signaling from cell membrane to the nucleus. Cell 113, 685-700 (2003).

147. Siegel, P. M. \& Massagué, J. Cytostatic and apoptotic actions of TGF- $\beta$ in homeostasis and cancer. Nat. Rev. Cancer 3, 807-821 (2003). 
148. Simonsson, M., Kanduri, M., Grönroos, E., Heldin, C. H. \& Ericsson, J. The DNA binding activities of $\mathrm{Smad} 2$ and $\mathrm{Smad} 3$ are regulated by coactivator-mediated acetylation. J. Biol. Chem. 281, 39870-39880 (2006).

149. Storey, J. M. \& Storey, K. B. Metabolic rate depression and biochemical adaptation in anaerobiosis, hibernation and estivation. Q. Rev. Biol. 65, 145-174 (1990).

150. Storey, K. B. Life in the slow lane: Molecular mechanisms of estivation. Comp. Biochem. Physiol. - A Mol. Integr. Physiol. 133, 733-754 (2002).

151. Storey, K. B. \& Storey, J. M. Aestivation: signaling and hypometabolism. J. Exp. Biol. 215, 1425-1433 (2012).

152. Storey, K. B. \& Storey, J. M. Metabolic rate depression: the biochemistry of mammalian hibernation. Adv. Clin. Chem. 52, 77-108 (2010).

153. Storey, K. B. \& Storey, J. M. Metabolic rate depression in animals: transcriptional and translational controls. Biol. Rev. 79, 207-233 (2004).

154. Suwanabol, P. A. et al. TGF- $\beta$ and Smad3 modulate PI3K/Akt signaling pathway in vascular smooth muscle cells. Am. J. Physiol. Hear. Circ. Physiol. 302, H2211H2219 (2012).

155. Tabruyn, S. P. \& Griffioen, A. W. Molecular pathways of angiogenesis inhibition. Biochem. Biophys. Res. Commun. 355, 1-5 (2007).

156. Tanaka, T. \& Nangaku, M. Angiogenesis and hypoxia in the kidney. Nat. Rev. Nephrol. 9, 211-222 (2013).

157. Tanos, T. et al. Phosphorylation of c-Fos by members of the p38 MAPK family: role of the AP-1 response to UV light. J. Biol. Chem. 280, 18842-18852 (2005).

158. ten Dijke, P. \& Arthur, H. M. Extracellular control of TGF- $\beta$ signalling in vascular development and disease. Nat. Rev. Mol. Cell Biol. 8, 857-869 (2007). 
159. ten Dijke, P., Goumans, M. J., Itoh, F. \& Itoh, S. Regulation of cell proliferation by Smad proteins. J. Cell. Physiol. 191, 1-16 (2002).

160. ten Dijke, P. \& Hill, C. S. New insights into TGF- $\beta$-Smad signalling. Trends Biochem. Sci. 29, 265-273 (2004).

161. Tkach, V. et al. Role of the Fos family members, c-Fos, Fra-1 and Fra-2, in the regulation of cell motility. Oncogene 22, 5045-5054 (2003).

162. Ultsch, G. R. The ecology of overwintering among turtles: where turtles overwinter and its consequences. Biol. Rev. Camb. Philos. Soc. 81, 339-67 (2006).

163. Ultsch, G. R. The viability of nearctic freshwater turtles submerged in anoxia and normoxia at 3 and 10 degrees C. Comp. Biochem. Physiol. 81, 607-611 (1985).

164. Ultsch, G. R. \& Jackson, D. C. Long-term submergence at 3 degrees C of the turtle Chrysemys picta bellii in normoxic and severely hypoxic water III. Effects of changes in ambient pO2 and subsequent air breathing. J. Exp. Biol. 97, 87-99 (1982).

165. Unsworth, B. R., Balinsky, J. B. \& Crook, E. M. Evidence for direct excretion of blood ammonia by an ammoniotelic amphibian. Comp. Biochem. Physiol. 31, 373377 (1969).

166. Vadysirisack, D. D. \& Ellisen, L. W. in mTOR: Methods and Protocols, Methods in Molecular Biology (eds. Walker, J. M. \& Weichhart, T.) 821, 45-58 (Humana Press, 2012).

167. van Meeteren, L. A. \& ten Dijke, P. Regulation of endothelial cell plasticity by TGFB. Cell Tissue Res. 347, 177-186 (2012).

168. Vassalli, J.-D. \& Stutz, A. Translational control: awakening dormant mRNAs. Curr. Biol. 5, 476-479 (1995). 
169. Verrecchia, F., Chu, M. L. \& Mauviel, A. Identification of novel TGF- $\beta /$ Smad gene targets in dermal fibroblasts using a combined cDNA microarray/promoter transactivation approach. J. Biol. Chem. 276, 17058-17062 (2001).

170. Wang, M. et al. Novel Smad proteins localize to IR-induced double-strand breaks: interplay between TGF- $\beta$ and ATM pathways. Nucleic Acids Res. 41, 933-942 (2013).

171. Wang, S. E., Wu, F. Y., Shin, I., Qu, S. \& Arteaga, C. L. Transforming growth factor $\beta$ (TGF- $\beta$ )-Smad target gene protein tyrosine phosphatase receptor type kappa is required for TGF- $\beta$ function. Mol. Cell. Biol. 25, 4703-4715 (2005).

172. Weinstein, M. et al. Smad proteins and hepatocyte growth factor control parallel regulatory pathways that converge on $\beta 1$-integrin to promote normal liver development. Mol. Cell. Biochem. 21, 5122-5131 (2001).

173. Weis, S. M. \& Cheresh, D. A. Tumor angiogenesis: molecular pathways and therapeutic targets. Nat. Med. 17, 1359-1370 (2011).

174. Wharton, K. \& Derynck, R. TGF- $\beta$ family signaling: novel insights in development and disease. Development 136, 3691-3697 (2009).

175. Wilczynska, A. \& Bushell, M. The complexity of miRNA-mediated repression. Cell Death Differ. 22, 22-33 (2015).

176. Willmore, W. G. \& Storey, K. B. Glutathione systems and anoxia tolerance in turtles. Am. J. Physiol. 273, R219-25 (1997).

177. Wong, Y.-C., Wang, X. \& Ling, M.-T. Id-1 expression and cell survival. Apoptosis 9, 279-289 (2004).

178. Wouters, B. G. \& Koritzinsky, M. Hypoxia signalling through mTOR and the unfolded protein response in cancer. Nat. Rev. Cancer 8, 851-864 (2008). 
179. Wrana, J. L. Regulation of Smad activity. Cell 100, 189-192 (2000).

180. Wrana, J. L. et al. TGF- $\beta$ signals through a heteromeric protein kinase receptor complex. Cell 71, 1003-1014 (1992).

181. Wrana, J. L. \& Attisano, L. The Smad pathway. Cytokine Growth Factor Rev. 11, $5-13(2000)$.

182. Wrighton, K. H., Lin, X. \& Feng, X.-H. Phospho-control of TGF- $\beta$ superfamily signaling. Cell Res. 19, 8-20 (2009).

183. Wrighton, K. H., Lin, X., Yu, P. B. \& Feng, X. H. Transforming growth factor $\beta$ can stimulate Smad1 phosphorylation independently of bone morphogenic protein receptors. J. Biol. Chem. 284, 9755-9763 (2009).

184. Wu, C.-W. Molecular Adaptations of Mammalian Hibernation: Roles of Metabolic Signaling Regulation in the Torpor-Arousal Cycle. (Carleton University, 2014).

185. Wu, L. \& Derynck, R. Essential role of TGF- $\beta$ signaling in glucose-induced cell hypertrophy. Dev. Cell 17, 35-48 (2009).

186. Yagi, K. et al. c-Myc is a downstream target of the Smad pathway. J. Biol. Chem. 277, 854-861 (2002).

187. Yamasaki, S. \& Anderson, P. Reprogramming mRNA translation during stress. Curr. Opin. Cell Biol. 20, 222-226 (2008).

188. Yancopoulos, G. D. et al. Vascular-specific growth factors and blood vessel formation. Nature 407, 242-248 (2000).

189. Yin, X. et al. ATF3, an adaptive-response gene, enhances TGF- $\beta$ signaling and cancer-initiating cell features in breast cancer cells. J. Cell Sci. 123, 3558-3565 (2010). 
190. Yoshida, K. \& Matsuzaki, K. Differential regulation of TGF- $\beta /$ Smad signaling in hepatic stellate cells between acute and chronic liver injuries. Front. Physiol. 3, 17 (2012).

191. Yoshida, T. et al. ATF3 protects against renal ischemia-reperfusion injury. J. Am. Soc. Nephrol. 19, 217-224 (2008).

192. Zhang, Y., Chang, C., Gehling, D. J., Hemmati-Brivanlou, A. \& Derynck, R. Regulation of Smad degradation and activity by Smurf2, an E3 ubiquitin ligase. PNAS 98, 974-979 (2001).

193. Zhang, Y.\& Derynck, R. Regulation of Smad signalling by protein associations and signalling crosstalk. Trends Cell Biol. 9, 274-279 (1999).

194. Zhang, Y., Feng, X.-H. \& Derynck, R. Smad3 and Smad4 cooperate with c-Jun/cFos to mediate TGF- $\beta$-induced transcription. Nature 394, 909-913 (1998). 
APPENDIX A

Animal Specific Protein Sequences 


\section{SMAD1 (Xenopus laevis): $52.4 \mathrm{kDa}$}

MNVTSLFSFTSPAVKRLLGWKQGDEEEKWAEKAVDALVKKLKKKKGAMEELEKALSCPGQPSNCVTIPRS LDGRLQVSHRKGLPHVIYCRVWRWPDLQS HHELKPLECCEY PFGSKQKEVC INPYHYKRVES PVLPPVLV PRHSEYNPQHSLLAQFRNLEPSEPHMPHNATFPDSFQQPNSHPF PHS PNS SY PNSPGSSSTYPHSPASSD PGSPFQIPADTP PPAYMP PEDQMTQDNSQPMDTNMMVPNI SQDINRADVQAVAYEEPKHWCSIVYYELNN RVGEAFHASSTSVLVDGFTDPSNNRNRFCLGLLSNVNRNST I ENTRRH IGKGVHLYYVGGEVYAECLSDS SIFVQSRNCNFHHGFHPTTVCKI PSGCSLKIFNNQEFAQLLAQSVNHGFETVYELTKMCT IRMS FVKGWG AEYHRQDVTSTPCWIEIHLHGPLQWLDKVLTQMGSPHNPISSVS

\section{SMAD1 (Chrysemys picta bellii): $52.3 \mathrm{kDa}$}

MNVTSLFSFTSPAVKRLLGWKQGDEEEKWAEKAVDALVKKLKKKKGAMEELEKALSCPGQPSNCVTIPRS LDGRLQVSHRKGLPHVIYCRVWRWPDLQSHHELKPLECCEF PFGSKQKEVCINPYHYKRVES PVLP PVLV PRHSEYNPQHSLLAQFRNLGQNEPHMPHNATFPDSFQQPNSHPFPHS PNSSY PNSPGSSNSTYPHSPASS DPGSPFQMPADT PP PAY LPPEDQMTQDGSQPMDTNMMAPS I P PEINRGDVQAVAYEEPKHWCS IVYYELN NRVGEAFHASSTSVLVDGFTDPSNNKNRFCLGLLSNVNRNST IENTRRHI GKGVHLYYVGGEVYAECLSD SS IFVQSRNCNYHHGFHPTTVCK I PSGCSLKI FNNQEFAQLLAQSVNHGFETVYELTKMCTIRMSFVKGW GAEYHRQDVTSTPCWIEIHLHGPLQWLDKVLTQMGSPHNPISSVS

\section{SMAD2 (Xenopus laevis): $52.3 \mathrm{kDa}$}

MSS I LPFTPPVVKRLLGWKKSASGTTGAGGGEQNGQEEKWCEKAVKSLVKKLKKTGQLDELEKAITTQNC NTKCVTIPSTCSEIWGLSTPNT I DQWDTTGLYSFSEQTRS LDGRLQVSHRKGLPHVIYSRLWRWPDLHSH HELKAVENCEYAFNLKKDEVCVNPYHYQRVETPVLP PVLVPRHTEI LTELPPLDDYTHS I PENTNF PAGI EPQSNY IPETPP PGY ISEDGETSDQQLNQSMDTGSPAELSPSTLSPVNHNLDLQPVTYSEPAFWCS IAYY ELNQRVGETFHASQPSLTVDGFTDPSNSERFCLGLLSNVNRNATVEMTRRH I GRGVRLYYIGGEVFAECL SDSA I FVQS PNCNQRYGWHPATVCKI PPGCNLKI FNNQEFAALLAQSVNQGFEAVYQLTRMCT IRMSFVK GWGAEYRRQTVTSTPCWIELHLNGPLQWLDKVLTQMGSPSVRCSSMS

\section{SMAD2, isoform X1 (Chrysemys picta bellii): $52.3 \mathrm{kDa}$}

MSS I LPFTPPVVKRLLGWKKSAGGSGGAGGGEQNGQEEKWCEKAVKSLVKKLKKTGQLDELEKAITTQNC NTKCVTIPSTCSEIWGLSTPNT I DQWDTTGLYSFSEQTRS LDGRLQVS HRKGLPHVIYCRLWRWPDLHSH HELKAIENCEYAFNLKKDEVCVNPYHYQRVETPVLPPVLVPRHTEILTELPPLDDYTHS I PENTNFPAGI EPQSNY IPETPP PGY ISEDGETSDQQLNQSMDTGS PAELS PSTLSPVNHSLDLQPVTYSEPAFWCS IAYY ELNQRVGETFHASQPSLTVDGFTDPSNSERFCLGLLSNVNRNATVEMTRRHIGRGVRLYYIGGEVFAECL SDSA I FVQS PNCNQRYGWHPATVCKI P PGCNLK I FNNQEFAALLAQSVNQGFEAVYQLTRMCT IRMSFVK GWGAEYRRQTVTSTPCWIELHLNGPLQWLDKVLTQMGSPSVRCSSMS 


\section{SMAD2, isoform X2 (Chrysemys picta bellii): $48.9 \mathrm{kDa}$}

MSS I LPFTPPVVKRLLGWKKSAGGSGGAGGGEQNGQEEKWCEKAVKSLVKKLKKTGQLDELEKAITTQNC NTKCVTI PRS LDGRLQVSHRKGLPHV I YCRLWRWPDLHS HHELKA IENCEYAFNLKKDEVCVNPYHYQRV ETPVLP PVLVPRHTEILTELPPLDDYTHS I PENTNFPAGIEPQSNY I PETPP PGYISEDGETS DQQLNQS MDTGS PAELSPSTLSPVNHSLDLQPVTYSEPAFWCS IAYYELNQRVGETFHASQPSLTVDGFTDPSNSER FCLGLLSNVNRNATVEMTRRH I GRGVRLYYIGGEVFAECLSDSA I FVQSPNCNQRYGWHPATVCKI PPGC NLKIFNNQEFAALLAQSVNQGFEAVYQLTRMCTIRMSFVKGWGAEYRRQTVTSTPCWIELHLNGPLQWLD KVLTQMGSPSVRCSSMS

\section{SMAD2, isoform X3 (Chrysemys picta bellii): $47.2 \mathrm{kDa}$}

MSS ILPFTPPVVKRLLGWKKSAGGSGGAGGGEQNGQEEKWCEKAVKSLVKKLKKTGQLDELEKAITTQNC NTKCVTIPSTCSEIWGLSTPNT I DQWDTTGLYSFSEQTRS LDGRLQVS HRKGLPHVIYCRLWRWPDLHSH HELKA IENCEYAFNLKKDEVCVNPYHYQRVETPVLP PVLVPRHTEILTELP P LDDYTHS I PENTNFPAGI EPQSNYIPETPPPGYISEDGETSDQQLNQSMDTGSPAELSPSTLSPVNHSLDLQPVTYSEPAFWCS IAYY ELNQRVGETFHASQPSLTVDGFTDPSNSERFCLGLLSNVNRNATVEMTRRH I GCNLKI FNNQEFAALLAQ SVNQGFEAVYQLTRMCT IRMS FVKGWGAEYRRQTVTSTPCWIELHLNGPLQWLDKVLTQMGS PSVRCSSM $\mathrm{S}$

\section{SMAD3 (Xenopus laevis): $48.0 \mathrm{kDa}$}

MSS I LPFTPP IVKRLLGWKKGEQNGQEEKWCEKAVKSLVKKLKKSGQLDELEKALTTQS ISTKCITIPRS LDGRLQVSHRKGLPHVIYCRLWRWPDLHSHHELRAMEVCEYAFSMKKDEVCVNPYHYQRVETPVLPPVLV PRNTEI PAEFPSLDDYSHS I PENTNF PAGIEPQINY I PETP P PGYLSEDGETS DQMNHS I DTGS PNLS PN SMSPAHSNMDLQPVTYCEPAFWCS ISYYELNQRVGETFHASQPSMTVDGFTDPSNSERFCLGLLSNVNRN AAVELTRRH I GRGVRLYYIGGEVFAECLSDNA I FVQSPNCNQRYGWHPATVCKI PPGCNLKI FNNQEFAA LLAQSVNQGFEAVYQLTRMCT IRMSFVKGWGAEYRRQTVTSTPCWIELHLNGPLQWLDKVLTQMGSPS IR CSSVS

\section{SMAD3, isoform X1 (Chrysemys picta bellii):48.3 kDa}

MSS I LPFTPP IVKRLLGWKKGEQNGQEEKWCEKAVKSLVKKLKKTGQLDELEKAITTQNINTKCITIPRS LDGRLQVSHRKGLPHVIYCRLWRWPDLHSHHELRAMEMCEYAFNMKKDEVCVNPYHYQRVETPVLPPVLV PRHTEI PAEFPPLDDYSHS I PENTNEPAGIEPQSNY IPETPPPGYLSEDGETSDHQMNHSMDAGS PNLSP NPMS PAHNNLDLQPVTYCEPAFWCSISYYELNQRVGETFHASQPSMTVDGFTDPSNSERFCLGLLSNVNR NAAVELTRRH I GRGVRLYYIGGEVFAECLSDSAIFVQS PNCNQRYGWHPATVCKIP PGCNLKIFNNQEFA ALLAQSVNQGFEAVYQLTRMCTIRMSFVKGWGAEYRRQTVTSTPCWIELHLNGPLQWLDKVLTQMGSPS I RCSSVS

\section{SMAD3, isoform X2 (Chrysemys picta bellii): $42.1 \mathrm{kDa}$}

MKSQNTETFNGVKRSLDGRLQVSHRKGLPHVIYCRLWRWPDLHSHHELRAMEMCEYAFNMKKDEVCVNPY HYQRVETPVLPPVLVPRHTEI PAEFPPLDDYSHS I PENTNF PAGIEPQSNY I PETPPPGYLSEDGETSDH QMNHSMDAGS PNLS PNPMSPAHNNLDLQPVTYCEPAFWCS ISYYELNQRVGETFHASQPSMTVDGFTDPS NSERFCLGLLSNVNRNAAVELTRRHIGRGVRLYYIGGEVFAECLSDSA IFVQS PNCNQRYGWHPATVCKI PPGCNLKIFNNQEFAALLAQSVNQGFEAVYQLTRMCTIRMSFVKGWGAEYRRQTVTSTPCWIELHLNGPL QWLDKVLTQMGSPSIRCSSVS 


\section{SMAD4, gene 1 (Xenopus laevis): $59.9 \mathrm{kDa}$}

MS ITNTPTSNDACLS IVHSLMCHRQGGESETFAKRAIES LVKKLKEKKDELDSLITAITTNGAHPSKCVT IQRTLDGRLQVAGRKGFPHVIYARLWRWPDLHKNELKHVKYCQYAFDLKCDSVCVNPYHYERVVSPGIDL SGLTLQSSAPSGLLVKDEYGHDYAEGQQS ISAGDGLSIQTIQHPPSSRSSTETYSNPALLVPSEPSTPNP TSFSS I PVPSTSQPASLLAATHNDGLLS IAPVPPQGTQNGFSAQPATYHHNSTTTWTGSRTAAYTPNMSH HPNGHLQHHP PMPHPGHYWPVHNELAFQP P ISNHPAPDYWCS IAYFEMDVQVGETFKVPS SCP IVTVDGY VDPSGGDRFCLGQLSNVHRTEAIERARLHIGKGVQLECKGEGDVWVRCLSDHAVFVQSYYLDREAGRAPG DAVHKIYPSAY IKVFDLRQCHRQMQQQAATAQAAAAAQAAAVAGNI PGPGSVGGIAPA ISLSAAAG IGVD DLRRLC ILRMS FVKGWGP DY PRQS IKETPCWIEIHLHRALQLLDEVLHTMP IADPQPLD

\section{SMAD4, gene 2 (Xenopus laevis): $61.3 \mathrm{kDa}$}

MS LTPPNSSDACLS IVHSLMCHRQGGENEGFAKRAIESLVKKLKEKKDELDSLITAITTNGVHPSKCVTI QRTLDGRLQVAGRKGFPHVIYARLWRWPDLHKNELKHVKFCQFAFDLKYDSVCVNPYHYERVVS PGIGLS IPSTVTTPCRSVKEEYVHECEMDASSCLPASQELPPAIKHASLPPMP PTESYRQPLPPLTLPKSPQTAIS MY PNMPLS PSVAPGCPLI PMHGEGLLQIAPSH PQQMLS IS P PST PSQNSQQNGYSS P PKQPFHASWTGSS TAVYTPNPGVQQNGRGNQQP PLHHPNNYWPLHQSSPQYQHPVSNHPGPEFWCSVAYFEMDVQVGEIFKVP SNCPVVTVDGYVDPSGGDRFCLGQLSNVHRTDTSERARLH I GKGVQLECRGEGDVWMRCLSDHAVFVQSY YLDREAGRAPGDAVHK IYPGAY IKVFDLRQCHRQMQQQAATAQAAAAAQAAAVAGAI PGPGSVGGIAPAV SLSAAAGIGVDDLRRLCI LRLSFVKGWGP DYPRQS IKQTPCWIEVHLHRALQLLDEVLHTLPMADPSSVN

\section{SMAD4 (Chrysemys picta bellii): $60.3 \mathrm{kDa}$}

MDNMS ITNTPTSNDACLS IVHS LMCHRQGGESETFAKRAIESLVKKLKEKKDELDSLITAITTNGAHPSK CVTIQRTLDGRLQVAGRKGFPHVIYARLWRWPDLHKNELKHVKYCQYAFDLKCDSVCVNPYHYERVVSPG IDLSGLTLQSSAPSSMLVKDEYVHDFEGQSLSTAEGHSVQTIQHPPSNRASTETYSTPAMLAPTESSTTS TTNFPNIPVASTSQPTS I LTGSHSDGLLQIASGPQPGTQQNGFTAQPATYHHNSTTTWTGSRTAAYTPTI PHHQNGHLQHHP PMPHPGHYWPVHNE LAFQPP I SNHPAPEYWCS IAYFEMDVQVGETFKVPSSCPVVTVD GYVDPSGGDRFCLGQLSNVHRTEAIERARLHIGKGVQLECKGEGDVWVRCLSDHAVFVQSYYLDREAGRA PGDAVHKIYPSAYIKVFDLRQCHRQMQQQAATAQAAAAAQAAAVAGNI PGPGSVGGIAPAISLSAAAGIG VDDLRRLCI LRMSFVKGWGPDYPRQS IKETPCWIEIHLHRALQLLDEVLHTMP IADPQPLD

\section{SMAD5 (Chrysemys picta bellii): $52.2 \mathrm{kDa}$}

MTSMASLFSFTS PAVKRLLGWKQGDEEEKWAEKAVDALVKKLKKKKGAMEELEKALSSPGQPSKCVTIPR SLDGRLQVSHRKGL PHVIYCRVWRWP DLQSHHELKPLDICEF PFGSKQKEVC INPYHYKRVES PVLPPVL VPRHSEFNPQHSLLVQFRNLSHNEPHMPQNATFPDSFQQPNSTPFSIS PNS PY P PS PVSSTYPSSPASSG PSS PFQLPADT P PPAYMP PEDQMGQDNSQSMDT SNTMI SQ I I PNI S SRDVQPVAYEEPKHWCS IVYYELN NRVGEAFHASSTSVLVDGFTDPSNNKNRFCLGLLSNVNRNST IENTRRHIGKGVHLYYVGGEVYAECLSD SS IFVQSRNCNYHHGFHPTTVCKI PSGCSLKIFNNQEFAQLLAQSVNHGFEAVYELTKMCTIRMSFVKGW GAEYHRQDVTSTPCWIEIHLHGPLQWLDKVLTQMGSPLNPISSVS 
Transforming growth factor beta-1 precursor (Chrysemys picta bellii): $44.6 \mathrm{kDa}$

MGQAMGPLFLFLLALLRLWASQALSTCKT I DMELVKRKRIEAIRGQ I LSKLRLDSP PQVEGGDAALLRLP EEVMS LYNSTRDMVRDMVRQQEAGKPRES PQDEYYAKEVHKF SMLPVKDARYATWEKTSQSTFFLFNASQ VRAQI S SEALLHRAELRMLPKPGTEQRVELYQHYGNGSWRYLDSRAVGQVPEEWLAFDVTEVVRQWLSGQ ETLEI FKLRVHCSCEDTCEELIVNIDGFEKKRGDLGTVSSTVQKLPYVLVMSTPLERASHLQSSRRRRAL DTDYCFGTEEKNCCVRPLFIDFRKDLHWKWIHEPKGYMANFCMGPCPYVWSADTQYSKVLALYNQHNPSA SAAPCCVPQALAPLPIVYYVGRKPKVEQLSNMIVSSCKCS

\section{Transforming growth factor beta-1 precursor (Xenopus laevis):44.2 kDa}

MEVLWMLLVLLVLHLSS LAMSLSTCKAVDMEEVRKRRIEAIRGQ I LSKLKLDKTPDVDSEKMTVPSEAIF LYNSTLEVIREKATREEEHVGHDQNIQDYYAKQVYRFES ITELEDHEFKFKFNASHVRENVGMNSLLHHA ELRMYKKQTDKNMDQRMELFWKYQENGTTHSRYLESKYITPVTDDEWMS FDVTKTVNEWLKRAEENEQFG LQPACKCPTPQAKDIDIEGFPALRGDLASLSSKENTKPYLMITSMPAERIDTVTSSRKKRGVGQEYCFGN NGPNCCVKPLY INFRKDLGWKW I HEPKGYEANYCLGNCPY IWSMDTQYSKVLS LYNQNNPGAS I S PCCVP DVLEPLPI IYYVGRTAKVEQLSNMVVRSCNCS

\section{c-Fos Proto-oncogene Protein (Xenopus laevis):39.7 kDa}

MYHAFSSSTEYDAASSRCSSASPAGDSLTYYPSPAASFSSMGSPVSPQDFGGDSSSSFVPTVTAISTSPD LQWLVQPTLISSVAPSQSRAHPYGSTPAYSRSSVMKGSAGRGQSLGRRGKMEQLSPEEEEKRKVRRERNK MAAAKCRNRRRELTDTLQAETDDLEDQKSALQAE I AGLLKEKEKLEF I LAAHKPACK I PHDLDGAFQDLT SSLDLGLISETPCSSSSQEPVAEPLFPIGLSQSSMPEKENTQLQVSMELKSEPLDDFLFNSSHTGVTDAA RSVPDVDLTSSLYTSEWEPLYSTLSADMEPLCTPVVTCTPTCTTYTTSFVFTYPESDHF PNCGAAHRRGS SSNEQSSDSLNSPTLLAL

\section{c-Fos Proto-oncogene Protein (Chrysemys picta bellii): $40.4 \mathrm{kDa}$}

MMYQGFTTDYDASS SRCSSASPAGDSLTYYTSPADSFSSMGS PVNQQDFCTDLAVSSANFVPTVTAISTS PDLQWLVQPTLISSVAPSQNRAHPYGVPTS PASAYSRAGIVKSTGGRGQS I GRRGKIEQLSPEEEEKRRI RRERNKMAAAKCRNRRRELTDTLQAETDQLEEEKSTLQTE IANLLKEKEKLEF I LAAHRPACKI PEELQF SEELAVSSLDLSGLGEAPVPSPPESEEAAFALPMLQEAAQPEPLPEPPKASSSLELKPEPFDEFLFPHSS RETARSVPDMDLSGSSSFYTADWEPLAAGACGELEPLCTPVVTCTPCPSTYTSTFVFTYPEADSFPSCAA AHRKGSSSNEPSSDSLSSPTLLAL

\section{c-Myc Proto-oncogene Protein (Xenopus laevis): $47.3 \mathrm{kDa}$}

MPLNANFPSKNYDYDYDLQPCFFFLEEENFYHQQSRLQPPAPSEDIWKKFELLPTPPLSPSRRSSQSSLF PSTADQLEMVTEFLGGDMVNQS ICEADDEALLKS IVIQDCMWSGFSAAAKLEKVVSEKLASYQASRKES ALSSSSPCQSQPPPSPLKSPSCHGSLSLGGTHRSS HGFLQDPSS DCVDPSVVFPYPLNDS ISNASS PCQD LILETPPISSNSSSSESGEEPEDEDEDCDEEEE I DVVTVEKRQSASKRVESSSHSQPSRPHYSPLVLKRC HVP I HQHNYAAS PSTKVDYVSSKRAKLESNIRVLKQ ISNNRKCAS PRSS DSEENDKRKTHNVLERQRRNE LKLSFFALRDQVPEVASNEKAPKVVILKKATEYAISLQEDERRLIRETEQLKYRKEQLKQRLQQLRNFV 
c-Myc Proto-oncogene Protein, isoform X1 (Chrysemys picta bellii):45.6 kDa

MPLTSTLPSKNYDYDYDSVQPYFYFEEEEENFYLAAQQRGSELQPPAPSEDIWKKFELLPTPPLSPSRRS SLAAAYFPSTADQLEMVTELLGGDMVNQSFICDPDDESFVKS I I IQDCMWSGFSAAAKLEKVVSEKLASY QAARREGGSGSRLCPPPPPSQPPSLAPSPASASTYLHDLGAAASDCIDPSVVFPYPLSERSSKPGSPSSS PASLLGDDTPPTTSSDSEEEPEEDEEEIDVVTTSESESITQPTEEHSKPHHSPLVLKRCHVPIHQHNYAA PPSTKLEYPSAKRIKLDNGRVLKQISNNRKCSSPRTSDTEENDKRRTHNVLERQRRNELKLSFFALRDQI PEVANNEKAPKVVILKKATEYVLS IQTDEHRLIAEKEQLRKRREQLKHKLEQLRNSCA

c-Myc Proto-oncogene Protein, isoform X2 (Chrysemys picta bellii): $45.5 \mathrm{kDa}$

MPLTSTLPSKNYDYDYDSVQPYFYFEEEEENFYLAAQQRGSELQPPAPSEDIWKKFELLPTPPLSPSRRS SLAAAYFPSTADQLEMVTELLGGDMVNQSF ICDPDDESFVKS I I IQDCMWSGFSAAAKLEKVVSEKLASY QAARREGGSGSRLCPPPP PSQPPSLAPSPASASTYLHDLGAAASDCIDPSVVFPYPLSERSSKPGSPSSS PASLLGDDTPPTTSSDSEEPEEDEEEIDVVTTSESESITQPTEEHSKPHHSPLVLKRCHVPIHQHNYAAP PSTKLEYPSAKRIKLDNGRVLKQISNNRKCSSPRTSDTEENDKRRTHNVLERQRRNELKLSFFALRDQIP EVANNEKAPKVVILKKATEYVLS IQTDEHRLIAEKEQLRKRREQLKHKLEQLRNSCA 
APPENDIX B

Immunoblotting Conditions 
Table 1 - Immunoblotting conditions for experiments run on Trachemys scripta elegans liver tissues.

Trachemys scripta elegans: Liver

\begin{tabular}{|c|c|c|c|c|}
\hline $\begin{array}{l}\text { Target } \\
\text { Protein }\end{array}$ & $\begin{array}{c}\text { MW } \\
\text { (kDa) }\end{array}$ & $\begin{array}{l}\text { Blocking } \\
\text { Conditions } \\
\text { (time) }\end{array}$ & $\begin{array}{l}1^{\circ} \text { Antibody } \\
\text { Concentration } \\
\text { (time) }\end{array}$ & $\begin{array}{l}2^{\circ} \text { Antibody } \\
\text { Concentration } \\
\text { (time) }\end{array}$ \\
\hline SMAD1 & 52 & $\begin{array}{l}\text { HMW-PVA } \\
(1 \mathrm{~min})\end{array}$ & $\begin{array}{l}1: 1000 \mathrm{v} / \mathrm{v} \\
(\mathrm{O} / \mathrm{N})\end{array}$ & $\begin{array}{l}1: 8000 \mathrm{v} / \mathrm{v} \\
(45 \mathrm{~min})\end{array}$ \\
\hline SMAD2 & 52 & $\begin{array}{l}5 \% \mathrm{w} / \mathrm{v} \text { milk } \\
(30 \mathrm{~min})\end{array}$ & $\begin{array}{l}1: 1000 \mathrm{v} / \mathrm{v} \\
(\mathrm{O} / \mathrm{N})\end{array}$ & $\begin{array}{l}1: 5000 \mathrm{v} / \mathrm{v} \\
(20 \mathrm{~min})\end{array}$ \\
\hline p-SMAD2 & 52 & $\begin{array}{l}\text { HMW-PVA } \\
(1 \mathrm{~min})\end{array}$ & $\begin{array}{l}1: 1000 \mathrm{v} / \mathrm{v} \\
(\mathrm{O} / \mathrm{N})\end{array}$ & $\begin{array}{l}1: 8000 \mathrm{v} / \mathrm{v} \\
(45 \mathrm{~min})\end{array}$ \\
\hline SMAD3 & 48 & $\begin{array}{l}5 \% \mathrm{w} / \mathrm{v} \text { milk } \\
(30 \mathrm{~min})\end{array}$ & $\begin{array}{l}1: 1000 \mathrm{v} / \mathrm{v} \\
(\mathrm{O} / \mathrm{N})\end{array}$ & $\begin{array}{l}1: 5000 \mathrm{v} / \mathrm{v} \\
(20 \mathrm{~min})\end{array}$ \\
\hline p-SMAD3 & 48 & $\begin{array}{l}5 \% \mathrm{w} / \mathrm{v} \text { milk } \\
(30 \mathrm{~min})\end{array}$ & $\begin{array}{l}1: 1000 \mathrm{v} / \mathrm{v} \\
(\mathrm{O} / \mathrm{N})\end{array}$ & $\begin{array}{l}1: 5000 \mathrm{v} / \mathrm{v} \\
(20 \mathrm{~min})\end{array}$ \\
\hline SMAD4 & 60 & $\begin{array}{l}\text { HMW-PVA } \\
(1 \mathrm{~min})\end{array}$ & $\begin{array}{l}1: 1000 \mathrm{v} / \mathrm{v} \\
(\mathrm{O} / \mathrm{N})\end{array}$ & $\begin{array}{l}1: 8000 \mathrm{v} / \mathrm{v} \\
(45 \mathrm{~min})\end{array}$ \\
\hline SMAD5 & 52 & $\begin{array}{l}\text { HMW-PVA } \\
(1 \mathrm{~min})\end{array}$ & $\begin{array}{l}1: 1000 \mathrm{v} / \mathrm{v} \\
(\mathrm{O} / \mathrm{N})\end{array}$ & $\begin{array}{l}1: 8000 \mathrm{v} / \mathrm{v} \\
(45 \mathrm{~min})\end{array}$ \\
\hline p-SMAD1/5 & 52 & $\begin{array}{l}5 \% \mathrm{w} / \mathrm{v} \text { milk } \\
(30 \mathrm{~min})\end{array}$ & $\begin{array}{l}1: 1000 \mathrm{v} / \mathrm{v} \\
(\mathrm{O} / \mathrm{N})\end{array}$ & $\begin{array}{l}1: 5000 \mathrm{v} / \mathrm{v} \\
(20 \mathrm{~min})\end{array}$ \\
\hline $\begin{array}{l}\text { TGF- } \beta \text { I-III } \\
\text { Precursor }\end{array}$ & 45 & $\begin{array}{l}\text { HMW-PVA } \\
(1 \mathrm{~min})\end{array}$ & $\begin{array}{l}1: 1000 \mathrm{v} / \mathrm{v} \\
(2 \times \mathrm{O} / \mathrm{N})\end{array}$ & $\begin{array}{l}1: 8000 \mathrm{v} / \mathrm{v} \\
(45 \mathrm{~min})\end{array}$ \\
\hline
\end{tabular}


Table 2 - Immunoblotting conditions for experiments run on Trachemys scripta elegans white muscle tissues.

\section{Trachemys scripta elegans: White Muscle}

\begin{tabular}{|c|c|c|c|c|}
\hline $\begin{array}{l}\text { Target } \\
\text { Protein }\end{array}$ & $\begin{array}{c}\text { MW } \\
\text { (kDa) }\end{array}$ & $\begin{array}{l}\text { Blocking } \\
\text { Condition } \\
\text { (time) } \\
\end{array}$ & $\begin{array}{l}1^{\circ} \text { Antibody } \\
\text { Concentration } \\
\text { (time) }\end{array}$ & $\begin{array}{l}2^{\circ} \text { Antibody } \\
\text { Concentration } \\
\text { (time) }\end{array}$ \\
\hline SMAD1 & 52 & $\begin{array}{l}\text { HMW-PVA } \\
\text { (1 min) }\end{array}$ & $\begin{array}{l}1: 1000 \mathrm{v} / \mathrm{v} \\
(\mathrm{O} / \mathrm{N})\end{array}$ & $\begin{array}{l}1: 8000 \mathrm{v} / \mathrm{v} \\
(45 \mathrm{~min})\end{array}$ \\
\hline SMAD2 & 52 & $\begin{array}{l}5 \% \mathrm{w} / \mathrm{v} \text { milk } \\
(30 \mathrm{~min})\end{array}$ & $\begin{array}{l}1: 1000 \mathrm{v} / \mathrm{v} \\
(\mathrm{O} / \mathrm{N})\end{array}$ & $\begin{array}{l}1: 5000 \mathrm{v} / \mathrm{v} \\
(20 \mathrm{~min})\end{array}$ \\
\hline p-SMAD2 & 52 & $\begin{array}{l}5 \% \mathrm{w} / \mathrm{v} \text { milk } \\
(30 \mathrm{~min})\end{array}$ & $\begin{array}{l}1: 1000 \mathrm{v} / \mathrm{v} \\
(\mathrm{O} / \mathrm{N})\end{array}$ & $\begin{array}{l}1: 5000 \mathrm{v} / \mathrm{v} \\
(20 \mathrm{~min})\end{array}$ \\
\hline SMAD3 & 48 & $\begin{array}{l}5 \% \mathrm{w} / \mathrm{v} \text { milk } \\
(30 \mathrm{~min})\end{array}$ & $\begin{array}{l}1: 1000 \mathrm{v} / \mathrm{v} \\
(\mathrm{O} / \mathrm{N})\end{array}$ & $\begin{array}{l}1: 5000 \mathrm{v} / \mathrm{v} \\
(20 \mathrm{~min})\end{array}$ \\
\hline p-SMAD3 & 48 & $\begin{array}{l}5 \% \mathrm{w} / \mathrm{v} \text { milk } \\
(30 \mathrm{~min})\end{array}$ & $\begin{array}{l}1: 1000 \mathrm{v} / \mathrm{v} \\
(\mathrm{O} / \mathrm{N})\end{array}$ & $\begin{array}{l}1: 5000 \mathrm{v} / \mathrm{v} \\
(20 \mathrm{~min})\end{array}$ \\
\hline SMAD4 & 60 & $\begin{array}{l}5 \% \mathrm{w} / \mathrm{v} \text { milk } \\
(30 \mathrm{~min})\end{array}$ & $\begin{array}{l}1: 1000 \mathrm{v} / \mathrm{v} \\
(\mathrm{O} / \mathrm{N})\end{array}$ & $\begin{array}{l}1: 5000 \mathrm{v} / \mathrm{v} \\
(20 \mathrm{~min})\end{array}$ \\
\hline SMAD5 & 52 & $\begin{array}{l}\text { HMW-PVA } \\
\text { (1 min) }\end{array}$ & $\begin{array}{l}1: 1000 \mathrm{v} / \mathrm{v} \\
(\mathrm{O} / \mathrm{N})\end{array}$ & $\begin{array}{l}1: 8000 \mathrm{v} / \mathrm{v} \\
(45 \mathrm{~min})\end{array}$ \\
\hline p-SMAD1/5 & 52 & $\begin{array}{l}5 \% \mathrm{w} / \mathrm{v} \text { milk } \\
(30 \mathrm{~min})\end{array}$ & $\begin{array}{l}1: 1000 \mathrm{v} / \mathrm{v} \\
(\mathrm{O} / \mathrm{N})\end{array}$ & $\begin{array}{l}1: 5000 \mathrm{v} / \mathrm{v} \\
(20 \mathrm{~min})\end{array}$ \\
\hline $\begin{array}{l}\text { TGF- } \beta \text { I-III } \\
\text { Precursor }\end{array}$ & 45 & $\begin{array}{l}\text { HMW-PVA } \\
\text { (1 min) }\end{array}$ & $\begin{array}{l}1: 1000 \mathrm{v} / \mathrm{v} \\
(2 \times \mathrm{O} / \mathrm{N})\end{array}$ & $\begin{array}{l}1: 8000 \mathrm{v} / \mathrm{v} \\
(45 \mathrm{~min})\end{array}$ \\
\hline c-Мус & 47 & $\begin{array}{l}\text { HMW-PVA } \\
(1 \mathrm{~min})\end{array}$ & $\begin{array}{l}1: 1000 \mathrm{v} / \mathrm{v} \\
(2 \times \mathrm{O} / \mathrm{N})\end{array}$ & $\begin{array}{l}1: 8000 \mathrm{v} / \mathrm{v} \\
(45 \mathrm{~min})\end{array}$ \\
\hline
\end{tabular}


Table 3 - Immunoblotting conditions for experiments run on Trachemys scripta elegans red muscle tissues

Trachemys scripta elegans: Red Muscle

\begin{tabular}{|c|c|c|c|c|}
\hline $\begin{array}{l}\text { Target } \\
\text { Protein }\end{array}$ & $\begin{array}{c}\text { MW } \\
\text { (kDa) }\end{array}$ & $\begin{array}{l}\text { Blocking } \\
\text { Condition } \\
\text { (time) } \\
\end{array}$ & $\begin{array}{l}1^{\circ} \text { Antibody } \\
\text { Concentration } \\
\text { (time) }\end{array}$ & $\begin{array}{l}2^{\circ} \text { Antibody } \\
\text { Concentration } \\
\text { (time) }\end{array}$ \\
\hline SMAD1 & 52 & $\begin{array}{l}\text { HMW-PVA } \\
\text { (1 min) }\end{array}$ & $\begin{array}{l}1: 1000 \mathrm{v} / \mathrm{v} \\
(\mathrm{O} / \mathrm{N})\end{array}$ & $\begin{array}{l}1: 8000 \mathrm{v} / \mathrm{v} \\
(45 \mathrm{~min})\end{array}$ \\
\hline SMAD2 & 52 & $\begin{array}{l}\text { HMW-PVA } \\
(1.5 \mathrm{~min})\end{array}$ & $\begin{array}{l}1: 1000 \mathrm{v} / \mathrm{v} \\
(2 \times \mathrm{O} / \mathrm{N})\end{array}$ & $\begin{array}{l}1: 8000 \mathrm{v} / \mathrm{v} \\
(45 \mathrm{~min})\end{array}$ \\
\hline p-SMAD2 & 52 & $\begin{array}{l}\text { HMW-PVA } \\
(1 \mathrm{~min})\end{array}$ & $\begin{array}{l}1: 1000 \mathrm{v} / \mathrm{v} \\
(\mathrm{O} / \mathrm{N})\end{array}$ & $\begin{array}{l}1: 8000 \mathrm{v} / \mathrm{v} \\
(45 \mathrm{~min})\end{array}$ \\
\hline SMAD3 & 48 & $\begin{array}{l}\text { HMW-PVA } \\
(1 \mathrm{~min})\end{array}$ & $\begin{array}{l}1: 1000 \mathrm{v} / \mathrm{v} \\
(\mathrm{O} / \mathrm{N})\end{array}$ & $\begin{array}{l}1: 8000 \mathrm{v} / \mathrm{v} \\
(45 \mathrm{~min})\end{array}$ \\
\hline p-SMAD3 & 48 & $\begin{array}{l}\text { HMW-PVA } \\
(1.5 \mathrm{~min})\end{array}$ & $\begin{array}{l}1: 1000 \mathrm{v} / \mathrm{v} \\
(\mathrm{O} / \mathrm{N})\end{array}$ & $\begin{array}{l}1: 8000 \mathrm{v} / \mathrm{v} \\
(45 \mathrm{~min})\end{array}$ \\
\hline SMAD4 & 60 & $\begin{array}{l}\text { HMW-PVA } \\
\text { (1 min) }\end{array}$ & $\begin{array}{l}1: 1000 \mathrm{v} / \mathrm{v} \\
(\mathrm{O} / \mathrm{N})\end{array}$ & $\begin{array}{l}1: 8000 \mathrm{v} / \mathrm{v} \\
(45 \mathrm{~min})\end{array}$ \\
\hline SMAD5 & 52 & $\begin{array}{l}\text { HMW-PVA } \\
\text { (1 min) }\end{array}$ & $\begin{array}{l}1: 1000 \mathrm{v} / \mathrm{v} \\
(\mathrm{O} / \mathrm{N})\end{array}$ & $\begin{array}{l}1: 8000 \mathrm{v} / \mathrm{v} \\
(45 \mathrm{~min})\end{array}$ \\
\hline p-SMAD1/5 & 52 & $\begin{array}{l}\text { HMW-PVA } \\
(1.5 \mathrm{~min})\end{array}$ & $\begin{array}{l}1: 1000 \mathrm{v} / \mathrm{v} \\
(\mathrm{O} / \mathrm{N})\end{array}$ & $\begin{array}{l}1: 8000 \mathrm{v} / \mathrm{v} \\
(45 \mathrm{~min})\end{array}$ \\
\hline $\begin{array}{l}\text { TGF- } \beta \text { I-III } \\
\text { Precursor }\end{array}$ & 45 & $\begin{array}{l}\text { HMW-PVA } \\
\text { (1 min) }\end{array}$ & $\begin{array}{l}1: 1000 \mathrm{v} / \mathrm{v} \\
(2 \times \mathrm{O} / \mathrm{N})\end{array}$ & $\begin{array}{l}1: 8000 \mathrm{v} / \mathrm{v} \\
(45 \mathrm{~min})\end{array}$ \\
\hline c-Мyc & 47 & $\begin{array}{l}\text { HMW-PVA } \\
\text { (1 min) }\end{array}$ & $\begin{array}{l}1: 1000 \mathrm{v} / \mathrm{v} \\
(2 \times \mathrm{O} / \mathrm{N})\end{array}$ & $\begin{array}{l}1: 8000 \mathrm{v} / \mathrm{v} \\
(45 \mathrm{~min})\end{array}$ \\
\hline p-c-Мyc & 47 & $\begin{array}{l}\text { HMW-PVA } \\
\text { (1 min) }\end{array}$ & $\begin{array}{l}1: 1000 \mathrm{v} / \mathrm{v} \\
(5 \times \mathrm{O} / \mathrm{N})\end{array}$ & $\begin{array}{l}1: 8000 \mathrm{v} / \mathrm{v} \\
(45 \mathrm{~min})\end{array}$ \\
\hline
\end{tabular}


Table 4 - Immunoblotting conditions for experiments run on Trachemys scripta elegans kidney tissues.

\section{Trachemys scripta elegans: Kidney}

\begin{tabular}{|c|c|c|c|c|}
\hline $\begin{array}{l}\text { Target } \\
\text { Protein }\end{array}$ & $\begin{array}{c}\text { MW } \\
\text { (kDa) }\end{array}$ & $\begin{array}{l}\text { Blocking } \\
\text { Condition } \\
\text { (time) }\end{array}$ & $\begin{array}{l}1^{\circ} \text { Antibody } \\
\text { Concentration } \\
\text { (time) }\end{array}$ & $\begin{array}{l}2^{\circ} \text { Antibody } \\
\text { Concentration } \\
\text { (time) }\end{array}$ \\
\hline SMAD1 & 52 & $\begin{array}{l}\text { HMW-PVA } \\
(1.5 \mathrm{~min})\end{array}$ & $\begin{array}{l}1: 1000 \mathrm{v} / \mathrm{v} \\
(\mathrm{O} / \mathrm{N})\end{array}$ & $\begin{array}{l}1: 8000 \mathrm{v} / \mathrm{v} \\
(45 \mathrm{~min})\end{array}$ \\
\hline SMAD2 & 52 & $\begin{array}{l}\text { HMW-PVA } \\
(1.5 \mathrm{~min})\end{array}$ & $\begin{array}{l}1: 1000 \mathrm{v} / \mathrm{v} \\
(2 \times \mathrm{O} / \mathrm{N})\end{array}$ & $\begin{array}{l}1: 8000 \mathrm{v} / \mathrm{v} \\
(45 \mathrm{~min})\end{array}$ \\
\hline p-SMAD2 & 52 & $\begin{array}{l}\text { HMW-PVA } \\
(1.5 \mathrm{~min})\end{array}$ & $\begin{array}{l}1: 1000 \mathrm{v} / \mathrm{v} \\
(\mathrm{O} / \mathrm{N})\end{array}$ & $\begin{array}{l}1: 8000 \mathrm{v} / \mathrm{v} \\
(45 \mathrm{~min})\end{array}$ \\
\hline SMAD3 & 48 & $\begin{array}{l}\text { HMW-PVA } \\
(1 \mathrm{~min})\end{array}$ & $\begin{array}{l}1: 1000 \mathrm{v} / \mathrm{v} \\
(\mathrm{O} / \mathrm{N})\end{array}$ & $\begin{array}{l}1: 8000 \mathrm{v} / \mathrm{v} \\
(45 \mathrm{~min})\end{array}$ \\
\hline p-SMAD3 & 48 & $\begin{array}{l}\text { HMW-PVA } \\
(1.5 \mathrm{~min})\end{array}$ & $\begin{array}{l}1: 1000 \mathrm{v} / \mathrm{v} \\
(\mathrm{O} / \mathrm{N})\end{array}$ & $\begin{array}{l}1: 8000 \mathrm{v} / \mathrm{v} \\
(45 \mathrm{~min})\end{array}$ \\
\hline SMAD4 & 60 & $\begin{array}{l}\text { HMW-PVA } \\
(1.5 \mathrm{~min})\end{array}$ & $\begin{array}{l}1: 1000 \mathrm{v} / \mathrm{v} \\
(\mathrm{O} / \mathrm{N})\end{array}$ & $\begin{array}{l}1: 8000 \mathrm{v} / \mathrm{v} \\
(45 \mathrm{~min})\end{array}$ \\
\hline SMAD5 & 52 & $\begin{array}{l}\text { HMW-PVA } \\
(1 \mathrm{~min})\end{array}$ & $\begin{array}{l}1: 1000 \mathrm{v} / \mathrm{v} \\
(\mathrm{O} / \mathrm{N})\end{array}$ & $\begin{array}{l}1: 8000 \mathrm{v} / \mathrm{v} \\
(45 \mathrm{~min})\end{array}$ \\
\hline p-SMAD1/5 & 52 & $\begin{array}{l}\text { HMW-PVA } \\
(1.5 \mathrm{~min})\end{array}$ & $\begin{array}{l}1: 1000 \mathrm{v} / \mathrm{v} \\
(\mathrm{O} / \mathrm{N})\end{array}$ & $\begin{array}{l}1: 8000 \mathrm{v} / \mathrm{v} \\
(45 \mathrm{~min})\end{array}$ \\
\hline $\begin{array}{l}\text { TGF-ßI-III } \\
\text { Precursor }\end{array}$ & 45 & $\begin{array}{l}\text { HMW-PVA } \\
(1.5 \mathrm{~min})\end{array}$ & $\begin{array}{l}1: 1000 \mathrm{v} / \mathrm{v} \\
(2 \times \mathrm{O} / \mathrm{N})\end{array}$ & $\begin{array}{l}1: 8000 \mathrm{v} / \mathrm{v} \\
(45 \mathrm{~min})\end{array}$ \\
\hline
\end{tabular}


Table 5 - Immunoblotting conditions for experiments run on Trachemys scripta elegans heart tissues.

\section{Trachemys scripta elegans: Heart}

\begin{tabular}{|c|c|c|c|c|}
\hline $\begin{array}{l}\text { Target } \\
\text { Protein }\end{array}$ & $\begin{array}{c}\text { MW } \\
\text { (kDa) }\end{array}$ & $\begin{array}{l}\text { Blocking } \\
\text { Condition } \\
\text { (time) } \\
\end{array}$ & $\begin{array}{l}1^{\circ} \text { Antibody } \\
\text { Concentration } \\
\text { (time) }\end{array}$ & $\begin{array}{l}2^{\circ} \text { Antibody } \\
\text { Concentration } \\
\text { (time) }\end{array}$ \\
\hline SMAD1 & 52 & $\begin{array}{l}\text { HMW-PVA } \\
(1 \mathrm{~min})\end{array}$ & $\begin{array}{l}1: 1000 \mathrm{v} / \mathrm{v} \\
(\mathrm{O} / \mathrm{N})\end{array}$ & $\begin{array}{l}1: 8000 \mathrm{v} / \mathrm{v} \\
(45 \mathrm{~min})\end{array}$ \\
\hline SMAD2 & 52 & $\begin{array}{l}\text { HMW-PVA } \\
(1 \mathrm{~min})\end{array}$ & $\begin{array}{l}1: 1000 \mathrm{v} / \mathrm{v} \\
(\mathrm{O} / \mathrm{N})\end{array}$ & $\begin{array}{l}1: 8000 \mathrm{v} / \mathrm{v} \\
(45 \mathrm{~min})\end{array}$ \\
\hline p-SMAD2 & 52 & $\begin{array}{l}\text { HMW-PVA } \\
(1 \mathrm{~min})\end{array}$ & $\begin{array}{l}1: 1000 \mathrm{v} / \mathrm{v} \\
(\mathrm{O} / \mathrm{N})\end{array}$ & $\begin{array}{l}1: 8000 \mathrm{v} / \mathrm{v} \\
(45 \mathrm{~min})\end{array}$ \\
\hline SMAD3 & 48 & $\begin{array}{l}5 \% \text { milk } \\
(30 \mathrm{~min})\end{array}$ & $\begin{array}{l}1: 1000 \mathrm{v} / \mathrm{v} \\
(\mathrm{O} / \mathrm{N})\end{array}$ & $\begin{array}{l}1: 8000 \mathrm{v} / \mathrm{v} \\
(45 \mathrm{~min})\end{array}$ \\
\hline p-SMAD3 & 48 & $\begin{array}{l}\text { HMW-PVA } \\
(1 \mathrm{~min})\end{array}$ & $\begin{array}{l}1: 1000 \mathrm{v} / \mathrm{v} \\
(\mathrm{O} / \mathrm{N})\end{array}$ & $\begin{array}{l}1: 8000 \mathrm{v} / \mathrm{v} \\
(45 \mathrm{~min})\end{array}$ \\
\hline SMAD4 & 60 & $\begin{array}{l}\text { HMW-PVA } \\
(1 \mathrm{~min})\end{array}$ & $\begin{array}{l}1: 1000 \mathrm{v} / \mathrm{v} \\
(\mathrm{O} / \mathrm{N})\end{array}$ & $\begin{array}{l}1: 8000 \mathrm{v} / \mathrm{v} \\
(45 \mathrm{~min})\end{array}$ \\
\hline SMAD5 & 52 & $\begin{array}{l}\text { HMW-PVA } \\
(1 \mathrm{~min})\end{array}$ & $\begin{array}{l}1: 1000 \mathrm{v} / \mathrm{v} \\
(\mathrm{O} / \mathrm{N})\end{array}$ & $\begin{array}{l}1: 8000 \mathrm{v} / \mathrm{v} \\
(45 \mathrm{~min})\end{array}$ \\
\hline p-SMAD1/5 & 52 & $\begin{array}{l}\text { HMW-PVA } \\
(1 \mathrm{~min})\end{array}$ & $\begin{array}{l}1: 1000 \mathrm{v} / \mathrm{v} \\
(\mathrm{O} / \mathrm{N})\end{array}$ & $\begin{array}{l}1: 8000 \mathrm{v} / \mathrm{v} \\
(45 \mathrm{~min})\end{array}$ \\
\hline $\begin{array}{l}\text { TGF-ßI-III } \\
\text { Precursor }\end{array}$ & 45 & $\begin{array}{l}\text { HMW-PVA } \\
\text { (1 min) }\end{array}$ & $\begin{array}{l}1: 1000 \mathrm{v} / \mathrm{v} \\
(2 \times \mathrm{O} / \mathrm{N})\end{array}$ & $\begin{array}{l}1: 8000 \mathrm{v} / \mathrm{v} \\
(45 \mathrm{~min})\end{array}$ \\
\hline
\end{tabular}


Table 6 - Immunoblotting conditions for experiments run on Xenopus laevis liver tissues.

\section{Xenopus laevis: Liver}

\begin{tabular}{|c|c|c|c|c|}
\hline $\begin{array}{l}\text { Target } \\
\text { Protein }\end{array}$ & $\begin{array}{c}\text { MW } \\
\text { (kDa) }\end{array}$ & $\begin{array}{l}\text { Blocking } \\
\text { Condition } \\
\text { (time) } \\
\end{array}$ & $\begin{array}{l}1^{\circ} \text { Antibody } \\
\text { Concentration } \\
\text { (time) }\end{array}$ & $\begin{array}{l}2^{\circ} \text { Antibody } \\
\text { Concentration } \\
\text { (time) } \\
\end{array}$ \\
\hline SMAD1 & 52 & $\begin{array}{l}\text { LMW-PVA } \\
(45 \mathrm{sec})\end{array}$ & $\begin{array}{l}1: 1000 \mathrm{v} / \mathrm{v} \\
(\mathrm{O} / \mathrm{N})\end{array}$ & $\begin{array}{l}1: 8000 \mathrm{v} / \mathrm{v} \\
(45 \mathrm{~min})\end{array}$ \\
\hline SMAD2 & 52 & $\begin{array}{l}\text { LMW-PVA } \\
(45 \mathrm{sec})\end{array}$ & $\begin{array}{l}1: 1000 \mathrm{v} / \mathrm{v} \\
(\mathrm{O} / \mathrm{N})\end{array}$ & $\begin{array}{l}1: 8000 \mathrm{v} / \mathrm{v} \\
(45 \mathrm{~min})\end{array}$ \\
\hline p-SMAD2 & 52 & $\begin{array}{l}\text { LMW-PVA } \\
(45 \mathrm{sec})\end{array}$ & $\begin{array}{l}1: 1000 \mathrm{v} / \mathrm{v} \\
(\mathrm{O} / \mathrm{N})\end{array}$ & $\begin{array}{l}1: 8000 \mathrm{v} / \mathrm{v} \\
(45 \mathrm{~min})\end{array}$ \\
\hline SMAD3 & 48 & $\begin{array}{l}8 \% \text { milk } \\
(\mathrm{O} / \mathrm{N})\end{array}$ & $\begin{array}{l}1: 1000 \mathrm{v} / \mathrm{v} \\
(\mathrm{O} / \mathrm{N})\end{array}$ & $\begin{array}{l}1: 8000 \mathrm{v} / \mathrm{v} \\
(45 \mathrm{~min})\end{array}$ \\
\hline p-SMAD3 & 48 & $\begin{array}{l}8 \% \text { milk } \\
(\mathrm{O} / \mathrm{N})\end{array}$ & $\begin{array}{l}1: 1000 \mathrm{v} / \mathrm{v} \\
(\mathrm{O} / \mathrm{N})\end{array}$ & $\begin{array}{l}1: 8000 \mathrm{v} / \mathrm{v} \\
(45 \mathrm{~min})\end{array}$ \\
\hline SMAD4 & 60 & $\begin{array}{l}\text { LMW-PVA } \\
(45 \mathrm{sec})\end{array}$ & $\begin{array}{l}1: 1000 \mathrm{v} / \mathrm{v} \\
(\mathrm{O} / \mathrm{N})\end{array}$ & $\begin{array}{l}1: 8000 \mathrm{v} / \mathrm{v} \\
(45 \mathrm{~min})\end{array}$ \\
\hline SMAD5 & 52 & $\begin{array}{l}\text { LMW-PVA } \\
(45 \mathrm{sec})\end{array}$ & $\begin{array}{l}1: 1000 \mathrm{v} / \mathrm{v} \\
(\mathrm{O} / \mathrm{N})\end{array}$ & $\begin{array}{l}1: 8000 \mathrm{v} / \mathrm{v} \\
(45 \mathrm{~min})\end{array}$ \\
\hline p-SMAD1/5 & 52 & $\begin{array}{l}\text { LMW-PVA } \\
(45 \mathrm{sec})\end{array}$ & $\begin{array}{l}1: 1000 \mathrm{v} / \mathrm{v} \\
(\mathrm{O} / \mathrm{N})\end{array}$ & $\begin{array}{l}1: 8000 \mathrm{v} / \mathrm{v} \\
(45 \mathrm{~min})\end{array}$ \\
\hline $\begin{array}{l}\text { TGF- } \beta \text { I-III } \\
\text { Precursor }\end{array}$ & 45 & $\begin{array}{l}\text { LMW-PVA } \\
(45 \mathrm{sec})\end{array}$ & $\begin{array}{l}1: 1000 \mathrm{v} / \mathrm{v} \\
(\mathrm{O} / \mathrm{N})\end{array}$ & $\begin{array}{l}1: 8000 \mathrm{v} / \mathrm{v} \\
(45 \mathrm{~min})\end{array}$ \\
\hline c-Fos & 40 & $\begin{array}{l}\text { LMW-PVA } \\
(45 \mathrm{sec})\end{array}$ & $\begin{array}{l}1: 1000 \mathrm{v} / \mathrm{v} \\
(2 \times \mathrm{O} / \mathrm{N})\end{array}$ & $\begin{array}{l}1: 8000 \mathrm{v} / \mathrm{v} \\
(45 \mathrm{~min})\end{array}$ \\
\hline p-c-Fos & 40 & $\begin{array}{l}\text { LMW-PVA } \\
(30 \mathrm{sec})\end{array}$ & $\begin{array}{l}1: 1000 \mathrm{v} / \mathrm{v} \\
(2 \times \mathrm{O} / \mathrm{N})\end{array}$ & $\begin{array}{l}1: 8000 \mathrm{v} / \mathrm{v} \\
(45 \mathrm{~min})\end{array}$ \\
\hline c-Myc & 47 & $\begin{array}{l}\text { LMW-PVA } \\
(45 \mathrm{sec})\end{array}$ & $\begin{array}{l}1: 1000 \mathrm{v} / \mathrm{v} \\
(2 \times \mathrm{O} / \mathrm{N})\end{array}$ & $\begin{array}{l}1: 8000 \mathrm{v} / \mathrm{v} \\
(45 \mathrm{~min})\end{array}$ \\
\hline p-c-Myc & 47 & $\begin{array}{l}\text { LMW-PVA } \\
(45 \mathrm{sec})\end{array}$ & $\begin{array}{l}1: 1000 \mathrm{v} / \mathrm{v} \\
(2 \times \mathrm{O} / \mathrm{N})\end{array}$ & $\begin{array}{l}1: 8000 \mathrm{v} / \mathrm{v} \\
(45 \mathrm{~min})\end{array}$ \\
\hline
\end{tabular}


Table 7 - Immunoblotting conditions for experiments run on Xenopus laevis muscle tissues.

\section{Xenopus laevis: Muscle}

\begin{tabular}{|c|c|c|c|c|}
\hline $\begin{array}{l}\text { Target } \\
\text { Protein }\end{array}$ & $\begin{array}{c}\text { MW } \\
\text { (kDa) }\end{array}$ & $\begin{array}{l}\text { Blocking } \\
\text { Condition } \\
\text { (time) }\end{array}$ & $\begin{array}{l}1^{\circ} \text { Antibody } \\
\text { Concentration } \\
\text { (time) }\end{array}$ & $\begin{array}{l}2^{\circ} \text { Antibody } \\
\text { Concentration } \\
\text { (time) }\end{array}$ \\
\hline SMAD1 & 52 & $\begin{array}{l}\text { LMW-PVA } \\
(45 \mathrm{sec})\end{array}$ & $\begin{array}{l}1: 1000 \mathrm{v} / \mathrm{v} \\
(\mathrm{O} / \mathrm{N})\end{array}$ & $\begin{array}{l}1: 8000 \mathrm{v} / \mathrm{v} \\
(45 \mathrm{~min})\end{array}$ \\
\hline SMAD2 & 52 & $\begin{array}{l}\text { LMW-PVA } \\
(45 \mathrm{sec})\end{array}$ & $\begin{array}{l}1: 1000 \mathrm{v} / \mathrm{v} \\
(\mathrm{O} / \mathrm{N})\end{array}$ & $\begin{array}{l}1: 8000 \mathrm{v} / \mathrm{v} \\
(45 \mathrm{~min})\end{array}$ \\
\hline p-SMAD2 & 52 & $\begin{array}{l}\text { LMW-PVA } \\
(45 \mathrm{sec})\end{array}$ & $\begin{array}{l}1: 1000 \mathrm{v} / \mathrm{v} \\
(\mathrm{O} / \mathrm{N})\end{array}$ & $\begin{array}{l}1: 8000 \mathrm{v} / \mathrm{v} \\
(45 \mathrm{~min})\end{array}$ \\
\hline SMAD3 & 48 & $\begin{array}{l}\text { LMW-PVA } \\
(45 \mathrm{sec})\end{array}$ & $\begin{array}{l}1: 1000 \mathrm{v} / \mathrm{v} \\
(\mathrm{O} / \mathrm{N})\end{array}$ & $\begin{array}{l}1: 8000 \mathrm{v} / \mathrm{v} \\
(45 \mathrm{~min})\end{array}$ \\
\hline p-SMAD3 & 48 & $\begin{array}{l}\text { LMW-PVA } \\
(45 \mathrm{sec})\end{array}$ & $\begin{array}{l}1: 1000 \mathrm{v} / \mathrm{v} \\
(\mathrm{O} / \mathrm{N})\end{array}$ & $\begin{array}{l}1: 8000 \mathrm{v} / \mathrm{v} \\
(45 \mathrm{~min})\end{array}$ \\
\hline SMAD4 & 59 & $\begin{array}{l}\text { LMW-PVA } \\
(45 \mathrm{sec})\end{array}$ & $\begin{array}{l}1: 1000 \mathrm{v} / \mathrm{v} \\
(\mathrm{O} / \mathrm{N})\end{array}$ & $\begin{array}{l}1: 8000 \mathrm{v} / \mathrm{v} \\
(45 \mathrm{~min})\end{array}$ \\
\hline SMAD5 & 52 & $\begin{array}{l}\text { LMW-PVA } \\
(45 \mathrm{sec})\end{array}$ & $\begin{array}{l}1: 1000 \mathrm{v} / \mathrm{v} \\
(\mathrm{O} / \mathrm{N})\end{array}$ & $\begin{array}{l}1: 8000 \mathrm{v} / \mathrm{v} \\
(45 \mathrm{~min})\end{array}$ \\
\hline p-SMAD1/5 & 52 & $\begin{array}{l}\text { LMW-PVA } \\
(45 \mathrm{sec})\end{array}$ & $\begin{array}{l}1: 1000 \mathrm{v} / \mathrm{v} \\
(\mathrm{O} / \mathrm{N})\end{array}$ & $\begin{array}{l}1: 8000 \mathrm{v} / \mathrm{v} \\
(45 \mathrm{~min})\end{array}$ \\
\hline $\begin{array}{l}\text { TGF- } \beta \text { I-III } \\
\text { Precursor }\end{array}$ & 45 & $\begin{array}{l}\text { HMW-PVA } \\
\text { (1 min) }\end{array}$ & $\begin{array}{l}1: 1000 \mathrm{v} / \mathrm{v} \\
(\mathrm{O} / \mathrm{N})\end{array}$ & $\begin{array}{l}1: 8000 \mathrm{v} / \mathrm{v} \\
(45 \mathrm{~min})\end{array}$ \\
\hline c-Fos & 40 & $\begin{array}{l}\text { LMW-PVA } \\
(45 \mathrm{sec})\end{array}$ & $\begin{array}{l}1: 1000 \mathrm{v} / \mathrm{v} \\
(2 \times \mathrm{O} / \mathrm{N})\end{array}$ & $\begin{array}{l}1: 8000 \mathrm{v} / \mathrm{v} \\
(45 \mathrm{~min})\end{array}$ \\
\hline p-c-Fos & 40 & $\begin{array}{l}\text { LMW-PVA } \\
(30 \mathrm{sec})\end{array}$ & $\begin{array}{l}1: 1000 \mathrm{v} / \mathrm{v} \\
(2 \times \mathrm{O} / \mathrm{N})\end{array}$ & $\begin{array}{l}1: 8000 \mathrm{v} / \mathrm{v} \\
(45 \mathrm{~min})\end{array}$ \\
\hline c-Myc & 47 & $\begin{array}{l}\text { LMW-PVA } \\
(45 \mathrm{sec})\end{array}$ & $\begin{array}{l}1: 1000 \mathrm{v} / \mathrm{v} \\
(2 \times \mathrm{O} / \mathrm{N})\end{array}$ & $\begin{array}{l}1: 8000 \mathrm{v} / \mathrm{v} \\
(45 \mathrm{~min})\end{array}$ \\
\hline p-c-Мyc & 47 & $\begin{array}{l}\text { LMW-PVA } \\
(45 \mathrm{sec})\end{array}$ & $\begin{array}{l}1: 1000 \mathrm{v} / \mathrm{v} \\
(2 \times \mathrm{O} / \mathrm{N})\end{array}$ & $\begin{array}{l}1: 8000 \mathrm{v} / \mathrm{v} \\
(45 \mathrm{~min})\end{array}$ \\
\hline
\end{tabular}




\section{APPENDIX C List of Antibodies}


Table 8 - List of antibodies, their properties, and details.

\begin{tabular}{|c|c|c|c|c|c|}
\hline Antigen & Type & Source & Clonality & Company & Catalog no. \\
\hline SMAD1 & Total & Rabbit & $\mathrm{pAb}$ & Cell Signaling & 9743 \\
\hline SMAD2 & Total & Rabbit & $\mathrm{mAb}$ & Cell Signaling & 5339 \\
\hline SMAD3 & Total & Rabbit & $\mathrm{mAb}$ & Cell Signaling & 9523 \\
\hline SMAD4 & Total & Rabbit & $\mathrm{pAb}$ & Cell Signaling & 9515 \\
\hline SMAD5 & Total & Rabbit & $\mathrm{pAb}$ & Cell Signaling & 9517 \\
\hline p-SMAD1/5 & Ser463/465 & Rabbit & $\mathrm{mAb}$ & Cell Signaling & 9516 \\
\hline p-SMAD2 & Ser465/467 & Rabbit & $\mathrm{mAb}$ & Cell Signaling & 3108 \\
\hline p-SMAD3 & Ser465/467 & Rabbit & $\mathrm{mAb}$ & Cell Signaling & 9520 \\
\hline c-Fos & Total & Rabbit & $\mathrm{pAb}$ & Santa Cruz & sc-052 \\
\hline p-c-Fos & Thr232 & Rabbit & $\mathrm{pAb}$ & Neo Biolabs & AP0038 \\
\hline с-Мус & Total & Rabbit & $\mathrm{pAb}$ & Santa Cruz & sc-764 \\
\hline p-c-Myc & Thr58/Ser62 & Rabbit & $\mathrm{pAb}$ & Santa Cruz & sc-8000-R \\
\hline $\begin{array}{l}\text { TGF- } \beta \text { I-III } \\
\text { Precursor }\end{array}$ & Total & Rabbit & $\mathrm{pAb}$ & Cell Signaling & 3711 \\
\hline BMP-2 & Total & Rabbit & $\mathrm{pAb}$ & Neo Biolabs & A0231 \\
\hline
\end{tabular}




\section{APPENDIX D \\ List of Epitopes of Specific Antibodies}


Table 9 - Epitopes of specific antibodies used in this study.

\begin{tabular}{l|l}
\hline Antigen & \multicolumn{1}{c}{ Epitope (made to Homo sapiens) } \\
\hline SMAD1 & Residues surrounding Ser190 of human Smad1 protein \\
SMAD2 & Residues near the N-terminus of mouse Smad2 protein \\
SMAD3 & Residues at the N-terminus of Smad3 protein \\
SMAD4 & Residues surrounding Ile251 of human Smad5 protein \\
SMAD5 & Residues surrounding Ser463/465 of human Smad5 protein \\
p-SMAD1/5 & Residues surrounding Ser465/467 of human Smad2 protein \\
p-SMAD2 & Residues surrounding Ser423/425 of Smad3 protein \\
p-SMAD3 & Residues surrounding the N-terminus of human c-Fos \\
TGF-BI-III Precursor & Residues surrounding T232 of human Fos \\
c-Fos & Residues 1-262 of human c-Myc \\
p-c-Fos & Ruman c-Myc \\
\hline
\end{tabular}


APPENDIX E

Ensuring Antibody Specificity and Band Selectivity for Immunoblotting 
Throughout this thesis, several steps were taken to ensure that the correct band was quantified for western blotting. These include:

1. Blocking with milk/PVA before probing with the antibody was optimized to minimize any non-specific binding and ensure that only one band appeared at the expected molecular weight.

2. Blots were run (at least once) with a mammalian control homogenate in addition to the turtle samples to ensure that bands cross-react at the same molecular weight.

3. If antibodies were tested on several different animals by the company, antibodies that were shown to cross-react well with a wide number of different species were selected. For example, the histone H3 antibody used cross-reacted with animals ranging from mammals to fruit flies.

4. If antibodies for different regions of the protein were available from the company, the amino acid sequences of these regions were aligned with sequences from several vertebrates and antibodies specific to the best conserved regions were selected.

5. Protein sequences were obtained from NCBI (http://www.ncbi.nlm.nih.gov) using specific keywords ('Latin animal' + 'protein name'). This sequence was fed into an online ExPASy tool (http://web.expasy.org) called 'Compute pI/Mw tool'. This tool was used to calculate the average molecular weight (MW) of a protein sequence to the precision of $0.01 \mathrm{Da}$. Bands were compared to this $\mathrm{MW}$ to ensure proper band selection. 


\section{APPENDIX F \\ Phylogenetic Trees of SMAD Proteins}




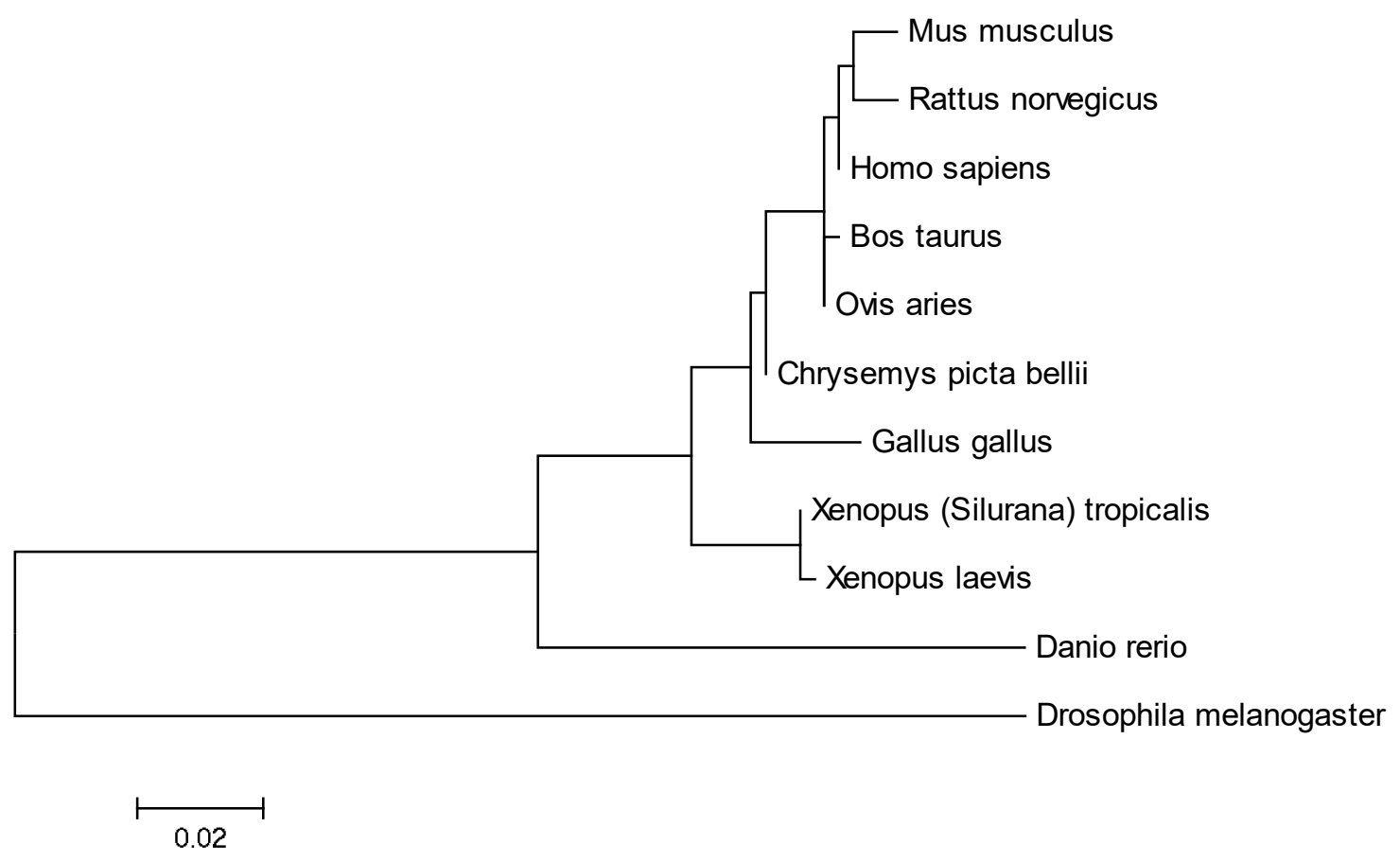

Figure F.1 - Molecular Phylogenetic analysis of SMAD1 protein by Maximum Likelihood method. The evolutionary history was inferred by using the Maximum Likelihood method based on the JTT matrix-based model (Jones et al., 1992). The tree with the highest log likelihood $(-2235.2848)$ is shown. Initial tree(s) for the heuristic search were obtained by applying the Neighbor-Joining method to a matrix of pairwise distances estimated using a JTT model. The analysis involved 11 amino acid sequences. All positions containing gaps and missing data were eliminated. There were a total of 439 positions in the final dataset. Evolutionary analyses were conducted in MEGA6 (Tamura et al., 2013). Species are: house mouse (Mus musculus), Norwegian rat (Rattus norvegicus), human (Homo sapiens), cow (Bos taurus), sheep (Ovis aries), Western painted turtle (Chrysemys picta bellii), chicken (Gallus gallus), Western clawed frog (Xenopus tropicalis), African clawed frog (Xenopus laevis), zebrafish (Danio rerio), and fruit fly (Drosophila melanogaster). 


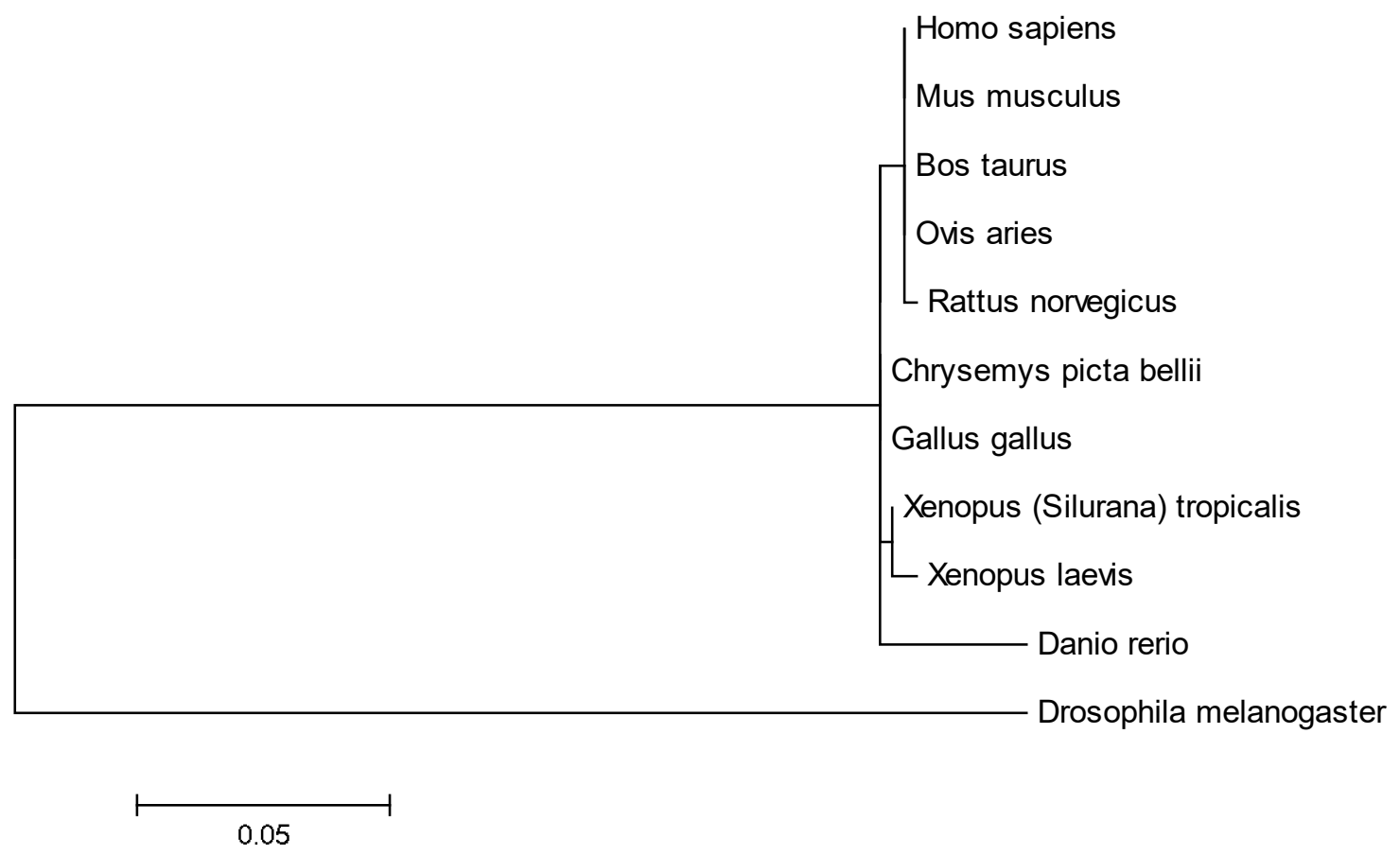

Figure F.2 - Molecular Phylogenetic analysis of SMAD2 protein by Maximum Likelihood method. The evolutionary history was inferred by using the Maximum Likelihood method based on the JTT matrix-based model (Jones et al., 1992). The tree with the highest log likelihood (-1927.4843) is shown. Initial tree(s) for the heuristic search were obtained by applying the Neighbor-Joining method to a matrix of pairwise distances estimated using a JTT model. The analysis involved 11 amino acid sequences. All positions containing gaps and missing data were eliminated. There were a total of 424 positions in the final dataset. Evolutionary analyses were conducted in MEGA6 (Tamura et al., 2013). Species are as in Figure F.1. 


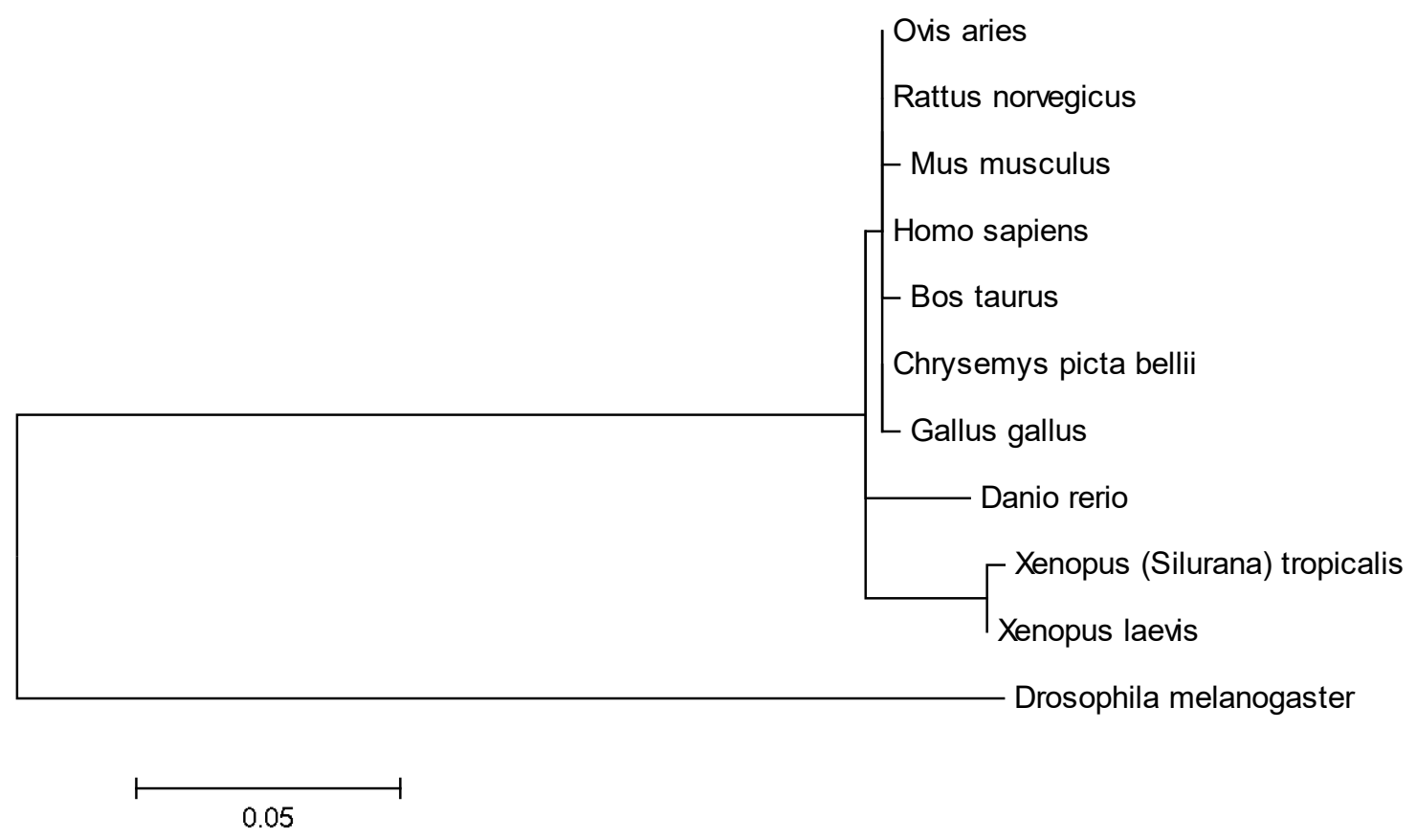

Figure F.3 - Molecular Phylogenetic analysis of SMAD3 protein by Maximum Likelihood method. The evolutionary history was inferred by using the Maximum Likelihood method based on the JTT matrix-based model (Jones et al., 1992). The tree with the highest log likelihood (-1435.8573) is shown. Initial tree(s) for the heuristic search were obtained automatically by applying Neighbor-Join and BioNJ algorithms to a matrix of pairwise distances estimated using a JTT model, and then selecting the topology with superior log likelihood value. The analysis involved 11 amino acid sequences. All positions containing gaps and missing data were eliminated. There were a total of 309 positions in the final dataset. Evolutionary analyses were conducted in MEGA6 (Tamura et al., 2013). Species are as in Figure F.1. 


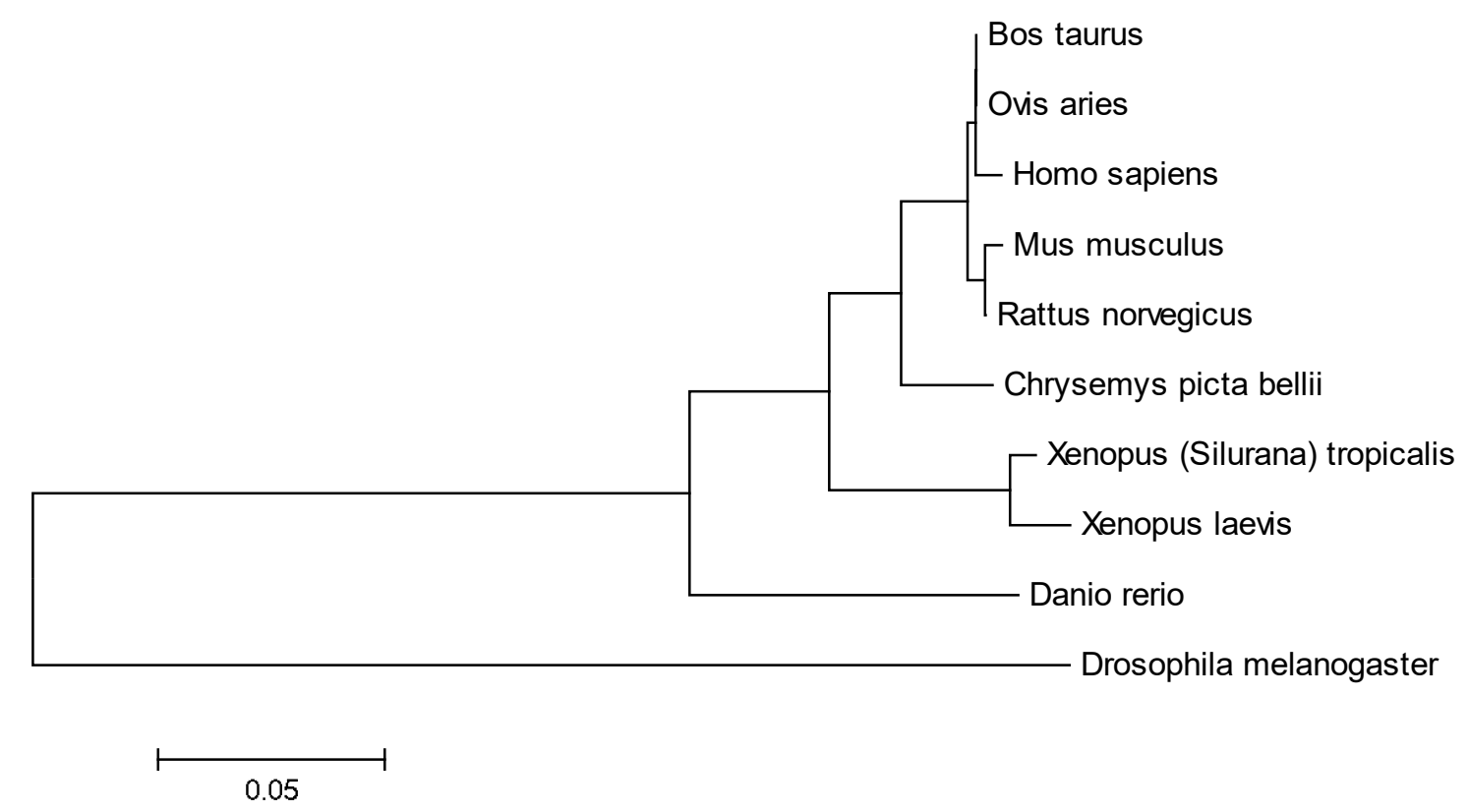

Figure F.4 - Molecular Phylogenetic analysis of SMAD 4 protein by Maximum Likelihood method. The evolutionary history was inferred by using the Maximum Likelihood method based on the JTT matrix-based model (Jones et al., 1992). The tree with the highest log likelihood (-2994.9171) is shown. Initial tree(s) for the heuristic search were obtained automatically by applying Neighbor-Join and BioNJ algorithms to a matrix of pairwise distances estimated using a JTT model, and then selecting the topology with superior log likelihood value. The analysis involved 10 amino acid sequences. All positions containing gaps and missing data were eliminated. There were a total of 528 positions in the final dataset. Evolutionary analyses were conducted in MEGA6 (Tamura et al., 2013). Species are as in Figure F.1. 


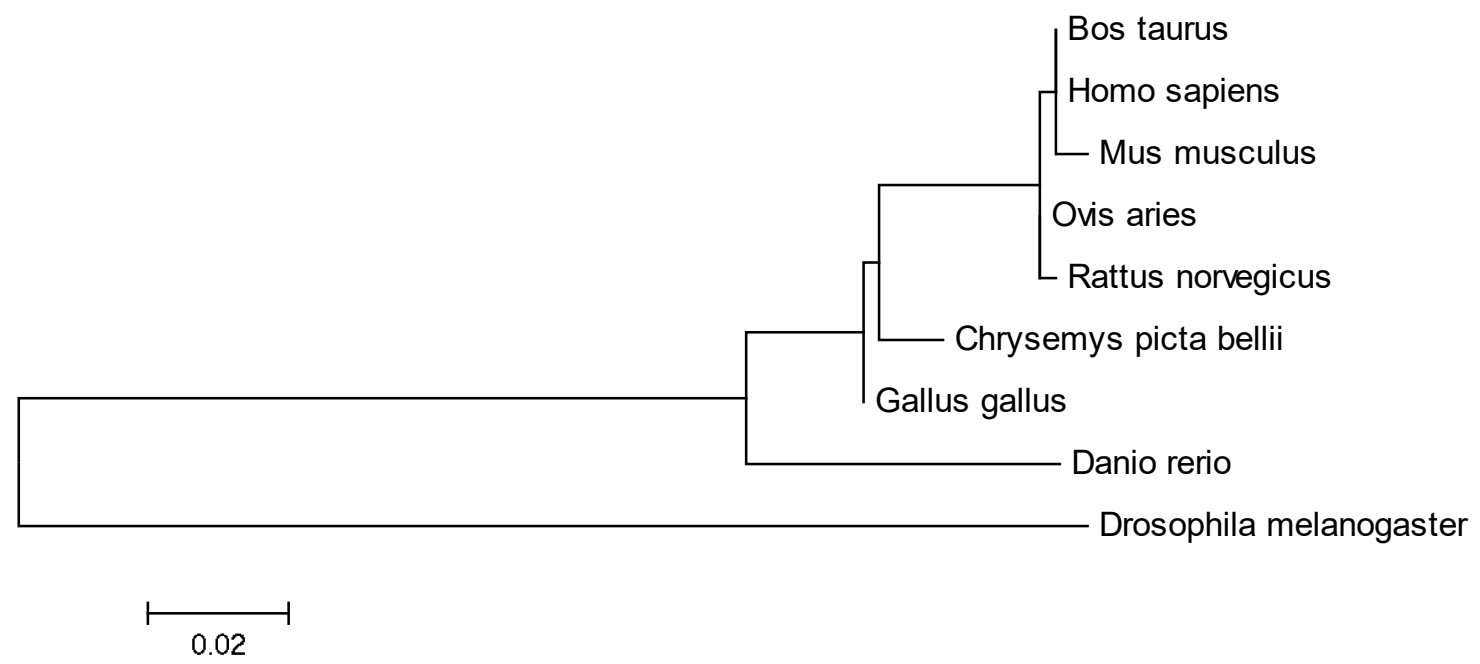

Figure F.5 - Molecular Phylogenetic analysis of SMAD5 protein by Maximum Likelihood method. The evolutionary history was inferred by using the Maximum Likelihood method based on the JTT matrix-based model (Jones et al., 1992). The tree with the highest log likelihood (-2061.1641) is shown. Initial tree(s) for the heuristic search were obtained by applying the Neighbor-Joining method to a matrix of pairwise distances estimated using a JTT model. The analysis involved 9 amino acid sequences. All positions containing gaps and missing data were eliminated. There were a total of 442 positions in the final dataset. Evolutionary analyses were conducted in MEGA6 (Tamura et al., 2013). Species are as in Figure F.1. 


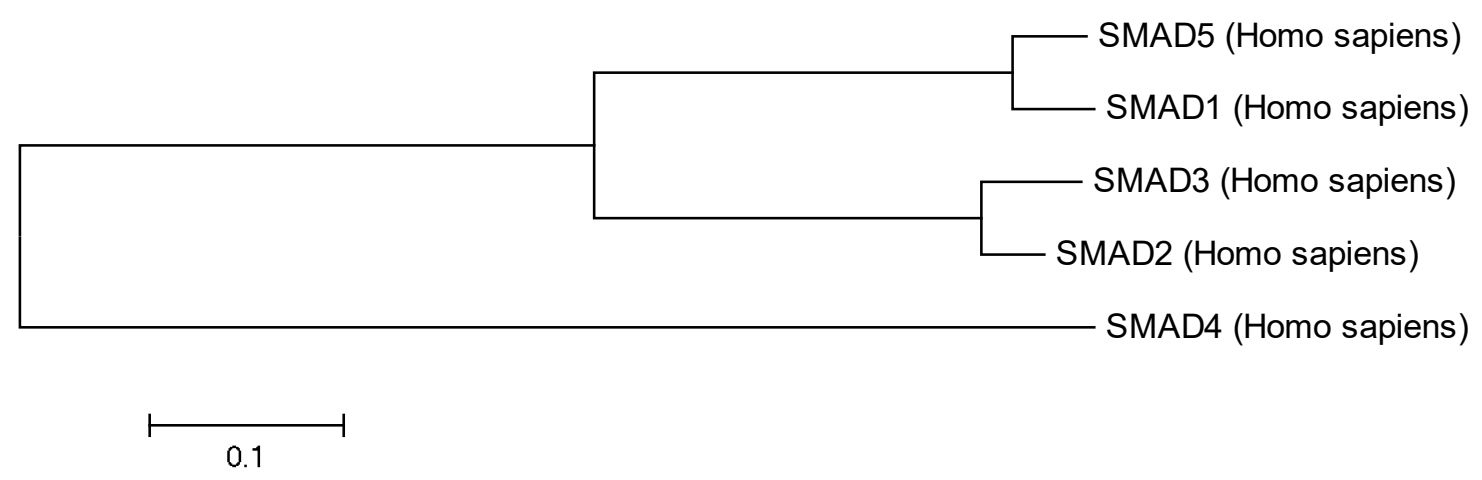

Figure F.6 - Molecular Phylogenetic analysis of SMAD proteins in Homo sapiens by Maximum Likelihood method. The evolutionary history was inferred by using the Maximum Likelihood method based on the JTT matrix-based model (Jones et al., 1992). The tree with the highest log likelihood (-3077.6095) is shown. Initial tree(s) for the heuristic search were obtained automatically by applying Neighbor-Join and BioNJ algorithms to a matrix of pairwise distances estimated using a JTT model, and then selecting the topology with superior log likelihood value. The analysis involved 5 amino acid sequences. All positions containing gaps and missing data were eliminated. There were a total of 417 positions in the final dataset. Evolutionary analyses were conducted in MEGA6 (Tamura et al., 2013). 


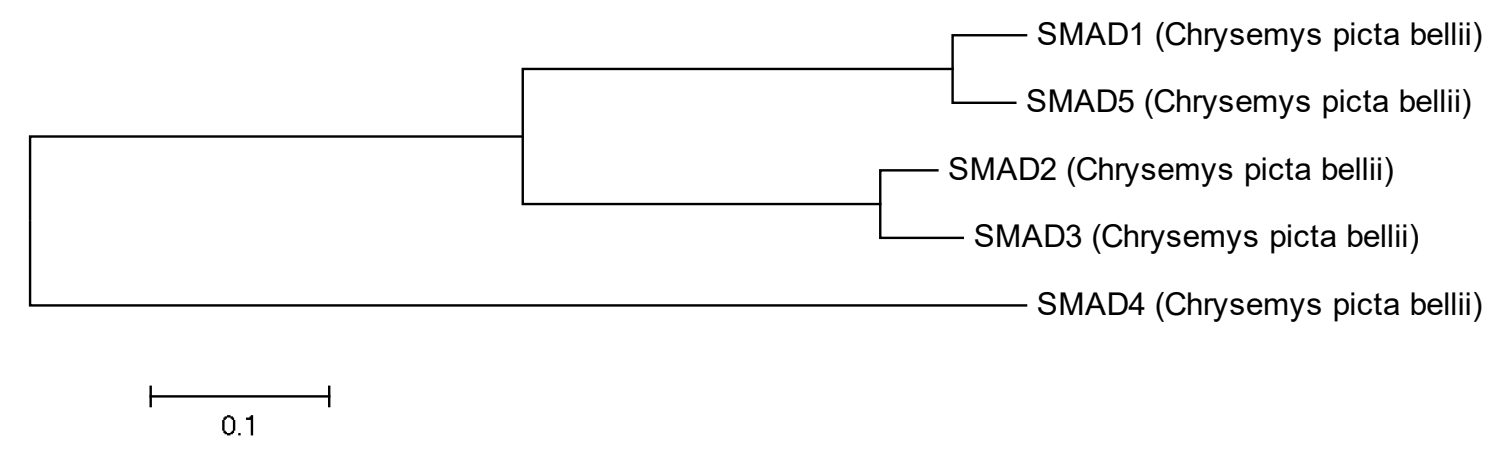

Figure F.7 - Molecular Phylogenetic analysis of SMAD proteins in Chrysemys picta bellii by Maximum Likelihood method. The evolutionary history was inferred by using the Maximum Likelihood method based on the JTT matrix-based model (Jones et al., 1992). The tree with the highest log likelihood (-3108.0581) is shown. Initial tree(s) for the heuristic search were obtained automatically by applying Neighbor-Join and BioNJ algorithms to a matrix of pairwise distances estimated using a JTT model, and then selecting the topology with superior log likelihood value. The tree is drawn to scale, with branch lengths measured in the number of substitutions per site. The analysis involved 5 amino acid sequences. All positions containing gaps and missing data were eliminated. There were a total of 420 positions in the final dataset. Evolutionary analyses were conducted in MEGA6 (Tamura et al., 2013). 
SMAD2 (Xenopus laevis) SMAD3 (Xenopus laevis) SMAD1 (Xenopus laevis) SMAD4 (Xenopus laevis)

0.1

Figure F.9 - Molecular Phylogenetic analysis of SMADs in Xenopus laevis by Maximum Likelihood method. The evolutionary history was inferred by using the Maximum Likelihood method based on the JTT matrix-based model (Jones et al., 1992). The tree with the highest log likelihood (-2965.1174) is shown. Initial tree(s) for the heuristic search were obtained automatically by applying Neighbor-Join and BioNJ algorithms to a matrix of pairwise distances estimated using a JTT model, and then selecting the topology with superior log likelihood value. The tree is drawn to scale, with branch lengths measured in the number of substitutions per site. The analysis involved 4 amino acid sequences. All positions containing gaps and missing data were eliminated. There were a total of 419 positions in the final dataset. Evolutionary analyses were conducted in MEGA6 (Tamura et al., 2013). 


\section{Appendix F References:}

1. Jones, D. T., Taylor, W. R. \& Thornton, J. M. The rapid generation of mutation

data matrices from protein sequences. Comput. Appl. Biosci. 8, 275-282 (1992).

2. Tamura, K., Stecher, G., Peterson, D., Filipski, A. \& Kumar, S. MEGA6:

Molecular evolutionary genetics analysis version 6.0. Mol. Biol. Evol. 30, 27252729 (2013). 
APPENDIX G

SMAD Isoforms: Roles and Controls 
A R-Smad

Smad1, Smad2, Smad3, Smad5, Smad8
Co-Smad

Smad4

\section{I-Smad \\ Smad6 \\ Smad7}
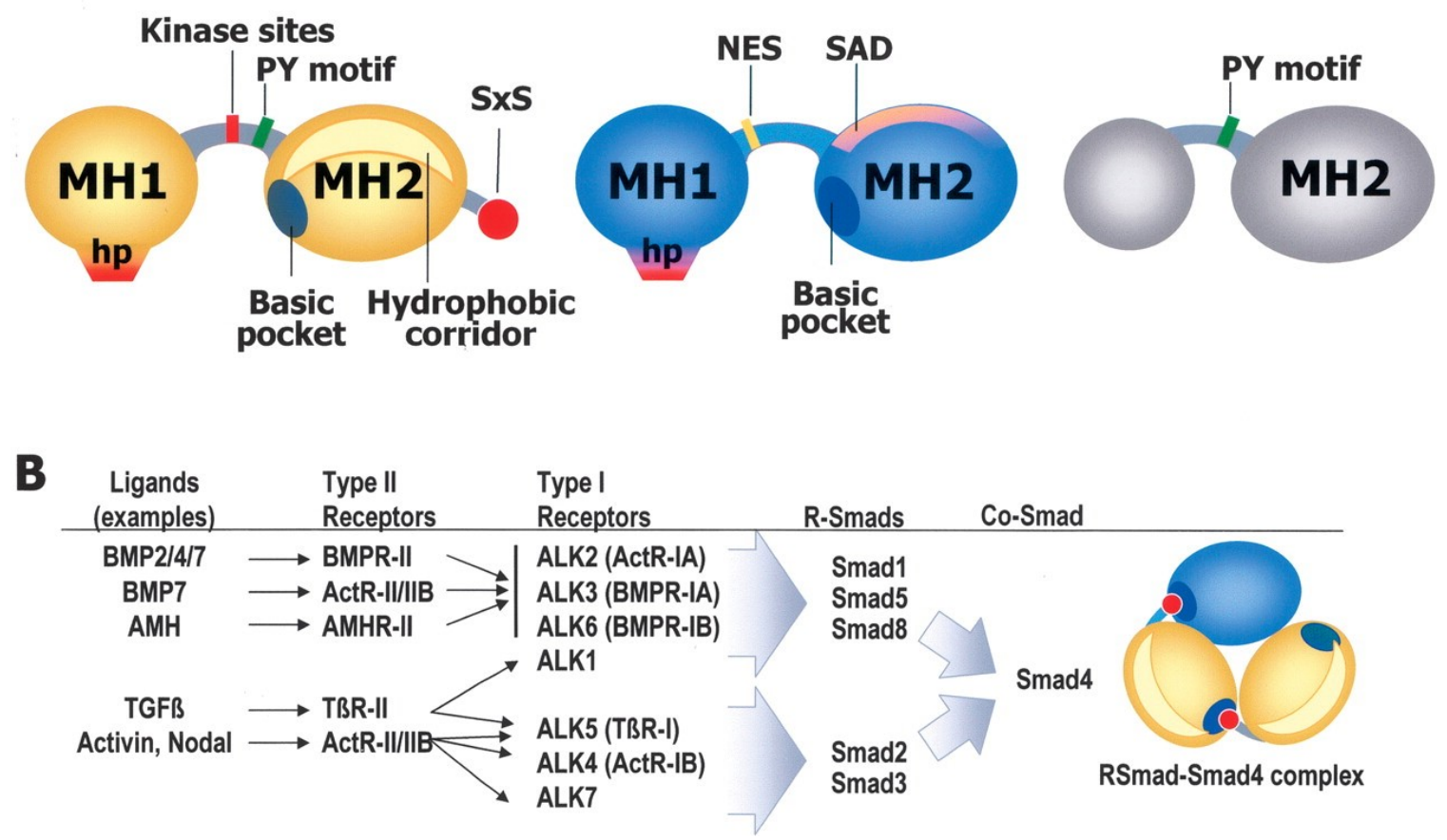
And I find it kinda funny

I find it kinda sad

The dreams in which I'm dying

Are the best I've ever had

I find it hard to tell you

I find it hard to take

When people run in circles

It's a very, very [Smad] world, [Smad] world

-Gary Jules, 'Mad World' (2001) 